\title{
Charakterisierung maßgeschneiderter makromolekularer Systeme: Analyse von photoschaltbaren Polymeren und Untersuchung der Wärmeleitfähigkeit von Polymeren und Polymernanokompositen
}

\author{
Dissertation \\ zur Erlangung des mathematisch-naturwissenschaftlichen \\ Doktorgrades \\ „Doctor rerum naturalium" \\ der Georg-August-Universität Göttingen \\ im Promotionsstudiengang Chemie \\ der Georg-August University School of Science (GAUSS) \\ vorgelegt von \\ Torsten Fornefeld \\ aus Gehrden
}

Göttingen, 2021 



\section{Betreuungsausschuss:}

Prof. Dr. Philipp Vana Institut für Physikalische Chemie Georg-August-Universität Göttingen

Prof. Dr. Marcus Müller Institut für Theoretische Physik Georg-August-Universität Göttingen

\section{Mitglieder der Prüfungskommission:}

\section{Referent}

Prof. Dr. Philipp Vana Institut für Physikalische Chemie

Korreferent

Prof. Dr. Marcus Müller Institut für Theoretische Physik Georg-August-Universität Göttingen

Weitere Mitglieder der Prüfungskommission:

Prof. Dr. Michael Buback Institut für Physikalische Chemie Georg-August-Universität Göttingen

Prof. Dr. Burkhard Geil Institut für Physikalische Chemie Georg-August-Universität Göttingen

Dr. Tim Schäfer

Institut für Physikalische Chemie Georg-August-Universität Göttingen

Prof. Dr. Thomas Zeuch Institut für Physikalische Chemie Georg-August-Universität Göttingen

Tag der mündlichen Prüfung: 13.04.2021 



\section{Zusammenfassung}

In dieser Arbeit wurden maßgeschneiderte photoschaltbare Polymere basierend auf dem Photoschalter Azobenzol synthetisiert und im Hinblick auf ihre Schaltkinetik, Mikrostruktur und Wärmeleitfähigkeit charakterisiert. Darüber hinaus erfolgte eine Untersuchung der Wärmeleitfähigkeit von Polymethylmethacrylat (PMMA), dem Blockcopolymer PMMA- $b$-Polybutylacrylat (PBA) und einem Nanokomposit aus PMMA und Gold-Nanopartikeln mit dem Ziel, Zusammenhänge zwischen Struktur des Materials und ihren Eigenschaften zu analysieren.

Auf Azobenzol aufbauend wurden verschiedene Methacrylat-basierte Monomere (AzoMA, AzoPMA, AzoHMA und AzoUMA) hergestellt, die sich in der Länge des Linkers zwischen Carbonylgruppe und Azobenzol unterscheiden. Die Kinetik der cis $\rightarrow$ trans-Isomerisierung in den photoschaltbaren Monomeren sowie in den zugehörigen durch reversible addition-fragmentation chain transfer (RAFT)-Polymerisation synthetisierten Polymeren wurde durch zeitaufgelöste UV/VisSpektroskopie in Lösung untersucht. Am Beispiel von AzoMA wurde gezeigt, dass die Aktivierungsenergie der Isomerisierung mit zunehmendem Dipolmoment des verwendeten Lösungsmittels abnimmt. Die Länge des verwendeten Linkers zeigte keinen Einfluss auf die Aktivierungsenergie. Die Geschwindigkeit des Schaltprozesses steigt hingegen mit Länge des eingesetzten Linkers an. Es konnte gezeigt werden, dass die Aktivierungsenergie für die Polymere geringer als für die Monomere ist. Darüber hinaus ist die Geschwindigkeit des Schaltprozesses für Polymere bei gleicher Temperatur höher als für die zugehörigen Monomere.

Von den photoschaltbaren Polymeren sowie von den daraus hergestellten Blockcopolymeren PAzoPMA- $b$-PBA wurden Dünnfilme hergestellt und durch Rasterkraftmikroskopie und Ellipsometrie untersucht. Für die photoschaltbaren Polymere konnte gezeigt werden, 
dass die Schichtdicke reversibel schaltbar ist. Die Blockcopolymere wurden hinsichtlich der Selbstanordnung ihrer Struktur charakterisiert. Die Analyse des zeitlichen Verlaufes ergab einen Übergang von einem ungeordneten Zustand zu einer lamellaren Morphologie der Mikrophasenseparation während des thermischen Temperns. Ein Vergleich von Blockcopolymeren mit PBA-Blöcken unterschiedlicher Länge zeigte, dass die Breite der PBA-Phase mit der Länge des PBA-Blockes zunimmt. Strukturparameter wie z. B. die Phasenbreiten konnten erfolgreich durch Beimischung der entsprechenden Homopolymere gezielt eingestellt werden. Mit PAzoPMA-b-Polydimethylaminoethylmethacrylat wurden photoschaltbare Aggregate in Propylencarbonat hergestellt. Diese konnten durch Photoschaltung in einer gesättigten KristallviolettLösung mit 8,2 Gew.-\% des Farbstoffes beladen werden.

Die Wärmeleitfähigkeit verschiedener makromolekularer Systeme wurde durch transiente Thermoreflektometrie bestimmt. Für PAzoPMA konnte eine Abnahme der Wärmeleitfähigkeit um $60 \%$ durch das Photoschalten des Polymers bestimmt werden. An einer Reihe von PMMA-Proben wurde gezeigt, dass die Wärmeleitfähigkeit für mittlere Molmassen zwischen 3,6 $\cdot 10^{3}$ und 1,6 $\cdot 10^{5} \mathrm{~g} / \mathrm{mol}$ keine Abhängigkeit von der Kettenlänge aufweist. Durch das Füllen einer Matrix aus PMMA mit 4,8 Gew.-\% PMMA-funktionalisierten GoldNanopartikeln konnte die Wärmeleitfähigkeit um $25 \%$ gesteigert werden. Durch unterschiedliche Temperverfahren (thermisch sowie durch Toluol-Atmosphäre) konnten die Mikrophasen in zylindrischer Morphologie eines PMMA- $b$-PBA-Blockcopolymers unterschiedlich ausgerichtet werden, wodurch gezeigt werden konnte, dass diese Strukturierung zu einer Anisotropie der Wärmeleitfähigkeit führt. Bei Messung senkrecht zur Zylinderanordnung wurde eine 40 \% höhere Wärmeleitfähigkeit als bei der Messung entlang der Zylinder erhalten. 


\section{Inhaltsverzeichnis}

1 Einleitung 1

2 Herstellung und Charakterisierung von photoschaltbaren Mono- und Polymeren 5

2.1 Hintergründe . . . . . . . . . . . . . . . . . . . 5

2.1.1 Die RAFT-Polymerisation . . . . . . . . . . . . . 5

2.1.2 Die Azobenzol-Gruppe als Photoschalter . . . . . 7

2.2 Synthese der photoschaltbaren Monomere . . . . . . . . 10

2.3 Thermische Relaxationskinetik von photoschaltbaren Monomeren und Polymeren . . . . . . . . . . . . . . . 12

2.3.1 Bestimmung des Geschwindigkeitskoeffizienten der thermischen Isomerisierung von AzoMA in Abhängigkeit des Lösungsmittels . . . . . . . . . 14

2.3.2 Synthese von photoschaltbaren Polymeren . . . . 17

2.3.3 Bestimmung des Geschwindigkeitskoeffizienten der thermischen Isomerisierung von Azobenzolhaltigen Monomeren und Polymeren in Abhängigkeit der Länge des verwendeten Linkers . . . . 18

2.4 Untersuchung von photoschaltbaren Polymerdünnfilmen 27

2.5 Fazit und weitere Forschungsperspektiven . . . . . . . 32

3 Untersuchung von photoschaltbaren Blockcopolymersystemen 35

3.1 Hintergründe . . . . . . . . . . . . . . . . . . 35

3.2 Synthese photoschaltbarer Blockcopolymere . . . . . . 41

3.3 Untersuchung der Mikrophasenseparation photoschaltbarer Blockcopolymere . . . . . . . . . . . . . . . . . 44

3.3.1 Zeitliche Untersuchung des Temperprozesses . . . 46

3.3.2 Untersuchung der Mikrophasenseparation von BCP II-IV . . . . . . . . . . . . . . . . . . . . . . . 48

3.4 Untersuchung der Mikrophasenseparation photoschaltbarer Blockcopolymer-Homopolymer-Blends . . . . . . . 55

3.5 Photospaltbare Blockcopolymeraggregate . . . . . . . . . 71 
3.6 Fazit und weitere Forschungsperspektiven . . . . . . . 74

4 Untersuchung der Wärmeleitfähigkeit makromolekularer Systeme $\quad 79$

4.1 Hintergründe . . . . . . . . . . . . . . . . . . . . 79

4.1.1 Einflüsse auf die Wärmeleitfähigkeit von Polymeren 85

4.2 Untersuchung der Wärmeleitfähigkeit photoschaltbarer Polymere . . . . . . . . . . . . . . . . . . 88

4.3 Wärmeleitfähigkeit von PMMA in Abhängigkeit von der Kettenlänge . . . . . . . . . . . . . . . . . . 9 90

4.4 Wärmeleitfähigkeit von Polymer-Gold-Nanokompositen 94

4.5 Wärmeleitfähigkeit von mikrophasenseparierten Blockcopolymeren . . . . . . . . . . . . . . . . . . . 98 98

4.6 Fazit und weitere Forschungsperspektiven . . . . . . . 105

5 Ausblick 109

6 Experimentalteil 113

6.1 Chemikalien . . . . . . . . . . . . . . . . . . . . 113

6.2 Monomere . . . . . . . . . . . . . . . . . . . 113

6.3 Initiatoren . . . . . . . . . . . . . . . . . . . . . 113

6.4 RAFT-Agens . . . . . . . . . . . . . . . . . . 113

6.5 Mess- und Analysegeräte . . . . . . . . . . . . . . . . . 114

6.6 Synthesen der photoschaltbaren Monomere . . . . . . . . 119

6.7 Synthesen der photoschaltbaren Polymere für die Untersuchung der Kinetik . . . . . . . . . . . . . . . 126

6.8 Synthesen der photoschaltbaren Blockcopolymere . . . . 128

6.9 Synthese von Homopolymeren zur Herstellung von Blockcopolymer-Homopolymer-Kompositen . . . . . . . 130

6.10 Herstellung des Blockcopolymers zur Herstellung von photospaltbaren, befüllten Polymeraggregaten . . . . . . 131

6.11 Herstellung der Polymere für die Wärmeleitfähigkeitsmessungen . . . . . . . . . . . . . . . . 132

6.12 Funktionalisierung der AuNP mit PMMA XI . . . . . . 133 
6.13 Synthese von BCP VI-IX . . . . . . . . . . . . . . . 133

7 Anhang 137

7.1 Herleitung der Gleichung für $c_{\text {cis }} \ldots \ldots$. . . . . . 137

7.2 Wertetabelle für die Dichten und Dipolmomente in Abbildung $15 \ldots \ldots \ldots$

7.3 AFM-Aufnahmen von BCP III . . . . . . . . . . . . . . . 139

7.4 AFM-Aufnahmen des DMT-Moduls von BCP I+20-50 . . 142

7.5 Flory-Fox-Auftragung für PMMA I-X . . . . . . . . . 144

7.6 Größenverteilung der Gold-Nanopartikel . . . . . . . . . 145

7.7 Berechnung des Massenanteils an Gold in den funktionalisierten AuNP . . . . . . . . . . . . . . . . . . . 146 



\section{Einleitung}

Schon vor vielen Jahrtausenden verwendete der Mensch neben Gesteinen, Metallen, Gläsern und Keramiken auch Polymere, die ihm die Natur zur Verfügung stellte. Solche Biopolymere wie Holz, Leder und Flachs waren für den Menschen essentiell. Um die Eigenschaften dieser Materialien zu verbessern, wurden sie weiterverarbeitet und modifiziert. Die ersten synthetischen Polymere sind auf den Beginn des 20. Jahrhunderts zu datieren. Polystyrol, Polymethylmethacrylat, Polyvinylchlorid, Polyethylen, Polypropylen sowie verschiedene Polyurethane werden auch heute noch vielfach genutzt. ${ }^{1} \mathrm{Um}$ die Jahrtausendwende lag der Fokus der Polymerchemie auf Hochleistungspolymeren für Spezialanwendungen: Flüssigkristalle und elektrisch leitfähige Polymere für Displays, faserverstärkte Polymere für Luft- und Raumfahrt sowie für Rennautos oder Rennräder, temperaturstabile Polymere für Isolierungen und Beschichtungen. Die Weltjahresproduktion an Polymeren steigt dabei stetig an. ${ }^{2}$

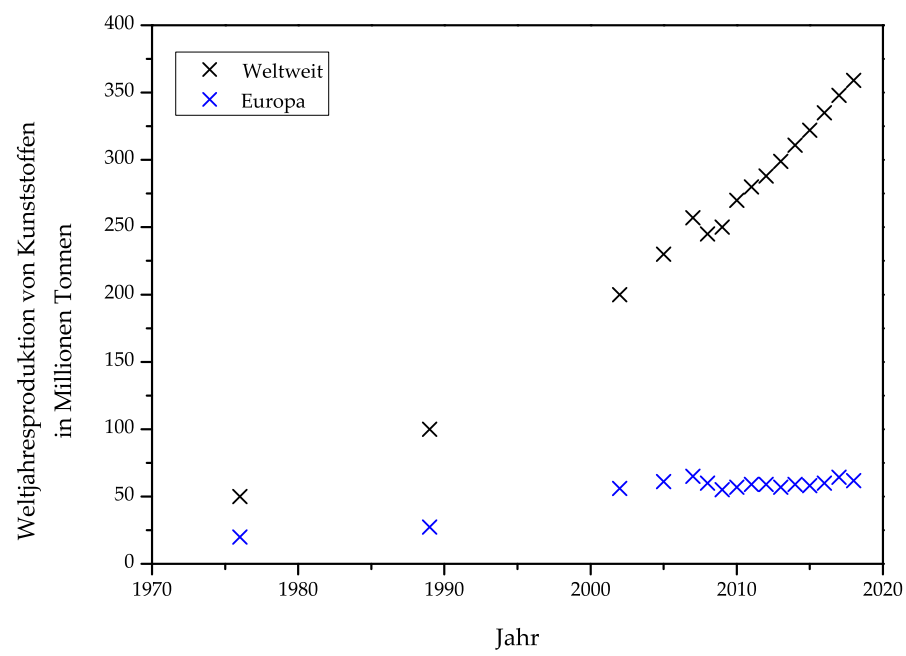

Abbildung 1: Kunststoffproduktion pro Jahr in Millionen Tonnen, weltweit und in Europa. 
Seit den 2010er-Jahren verstärkt sich der Nachhaltigkeitsgedanke der Bevölkerung, ${ }^{3-5}$ wodurch Gesetze verabschiedet werden, um die Verwendung von Einweg-Plastikartikeln zu vermeiden. ${ }^{6,7}$ Der Fokus der Polymerchemie verschiebt sich damit immer mehr von der Vewendung von Plastik als Verpackungsmaterial hin zur Entwicklung von Hochleistungspolymeren, die in ihren Eigenschaften natürlich vorkommenden Materialien überlegen und somit nicht zu ersetzen sind. Die Anforderungen an diese Werkstoffe sind dabei groß. Es werden maßgeschneiderte Materialien erwartet, die in der Nutzung eine optimale Leistung erzielen. Eine Möglichkeit, die Synthese derart zu steuern, sodass ein Polymer mit exakt den gewünschten Eigenschaften entsteht, liefert die reversible addition-fragmentation chain transfer-Polymerisation (RAFT-Polymerisation). ${ }^{8-12}$ Zusätzlich zur Auswahl des gewünschten Monomers erlaubt diese Polymerisationstechnik nicht nur die Kontrolle über die molare Masse des Polymers, es sind auch verschiedene Polymerarchitekturen zugänglich, welche entscheidend für die Materialeigenschaften sind. Die Herstellung von linearen, ${ }^{13-15}$ kammförmigen, ${ }^{16-18}$ sternförmigen, ${ }^{19-21}$ zyklischen ${ }^{22-24}$ oder auch vernetzten Polymeren ${ }^{25-27}$ ist möglich. Die Materialeigenschaften eines Polymers können durch die Zugabe von Additiven wie Weichmachern, Alterungsschutzmitteln, Farbstoffen oder Nanopartikeln angepasst werden. Für Hochleistungspolymere sind dabei insbesondere Nanokomposite von Interesse. Hierbei stehen verschiedene Materialien und Formen von Nanopartikeln zur Verfügung. ${ }^{28}$ Eine weitere Anpassungsmöglichkeit bietet die Einbringung einer schaltbaren Gruppe in das Polymer. Im Gegensatz zur Wahl eines Monomers oder der Verwendung von einer oder mehrerer Arten von Nanopartikeln können durch einen Schalter die Eigenschaften eines Polymers auch noch am Anwendungsort zwischen zwei Zuständen reversibel geschaltet werden. ${ }^{29}$ Unterschiedliche Schaltertypen können durch externe Reize wie Licht, pH-Wert, Temperatur, Ionen, Lösungsmittel oder Redoxreaktionen ausgelöst werden. ${ }^{30-32}$ Dabei bieten lichtinduziert-schaltende Gruppen einige Vorteile: Sie sind sowohl in Lösung, als auch im Festkörper nutzbar. Die Bestrahlung mit 
Licht ist nichtinvasiv und kann mit einer geeigneten Strahlungsquelle sehr präzise an der gewünschten Stelle eingesetzt werden. Bei Photoschaltern fallen zudem keine Abspalt- oder Nebenprodukte im Schaltprozess an.

Daraus ergeben sich die Kernpunkte dieser Arbeit: Mit Blick auf unterschiedliche Anwendungsbereiche werden photoschaltbare Polymere durch RAFT-Polymerisation hergestellt und charakterisiert. Die Schaltkinetik der Azobenzolgruppe wird dabei für verschiedene Mono- und Polymere im Hinblick auf Temperatur, Lösungsmittel und molekulare Umgebung untersucht. Des Weiteren wird der Einfluss des Photoschalters auf die Struktur von verschiedenen Homo- und Blockcopolymersystemen vorgestellt.

Zur Aufklärung von Struktur-Eigenschafts-Beziehungen von makromolekularen Systemen wird deren Wärmeleitfähigkeit bestimmt. Dabei werden Strukturparameter wie die Molmasse eines Polymers oder der Schaltzustand eines Photoschalters variiert. Zudem wird die Struktur durch Mikrophasenseparation eines Blockcopolymers oder das Einbringen von Nanopartikeln in eine Polymermatrix modifiziert. 


\section{Herstellung und Charakterisierung von photoschaltbaren Mono- und Polymeren}

\subsection{Hintergründe}

\subsubsection{Die RAFT-Polymerisation}

Die reversible addition-fragmentation chain transfer-Polymerisation (kurz: RAFT) gehört zur Klasse der reversibel-desaktivierenden radikalischen Polymerisationen. Sie unterscheidet sich von der konventionellen radikalischen Polymerisation mit Monomer $\mathrm{M}$, Initiator $\mathrm{I}_{2}$ und gegebenenfalls Lösungsmittel durch die Zugabe eines sogenannten RAFT-Agens zur Kontrolle der Reaktion. Die Vorteile gegenüber der konventionellen radikalischen Polymerisation liegen in der Toleranz funktioneller Gruppen, der Vielzahl der einsetzbaren Monomere und Lösungsmittel, sowie variablen Reaktionsbedingungen. Zudem ist eine Kontrolle über die mittlere Molmasse des Polymers $\left(M_{\mathrm{n}}\right)$ möglich und das Polymer weist eine enge Molmassenverteilung auf. Das RAFT-Agens bleibt nach Abschluss der Polymerisation an der Kette, sodass Makro-RAFT-Agenzien vorliegen die in weiteren Reaktionen zu (Multi-)Blockcopolymeren umgesetzt werden können. Werden RAFT-Agenzien mit mehreren RAFT-Gruppen eingesetzt, sind auch komplexe Polymertopologien wie Stern- oder Kammpolymere zugänglich.

Als RAFT-Agenzien werden in der Regel Dithioester oder Trithiocarbonat-Verbindungen eingesetzt. Diese tragen eine Abgangsgruppe $\mathrm{R}$, sowie eine stabilisierende Z-Gruppe.

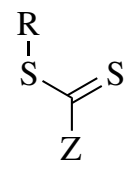

Abbildung 2: Allgemeine Struktur eines RAFT-Agens.

Im RAFT-Vorgleichgewicht wird die R-Gruppe durch die Anlagerung eines Polymerradikals $\mathrm{P}_{\mathrm{m}}{ }^{\bullet}$ abgespalten. Das entstandene Radikal $\mathrm{R}^{\bullet}$ 
muss in der Lage sein, ein Kettenwachstum zu initiieren. Es weist daher häufig eine strukturelle Ähnlichkeit zum Initiatorfragment I• auf. Die Z-Gruppe stabilisiert das intermediäre RAFT-Radikal. Zudem polarisiert sie die Kohlenstoff-Schwefel-Bindung im RAFT-Agens und macht sie so reaktiver. ${ }^{8-12}$ Der Mechanismus der RAFT-Polymerisation ähnelt dem der konventionellen radikalischen Polymerisation und ist in Schema 1 gezeigt.

Start:

$$
\mathrm{I}_{2} \longrightarrow \mathrm{I}^{\cdot} \stackrel{\mathrm{M}}{\longrightarrow} \mathrm{P}_{\mathrm{m}}^{\cdot}
$$

Vorgleichgewicht:

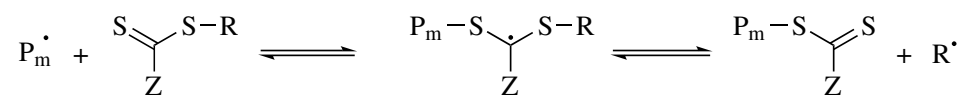

Reinitiierung:

$$
\mathrm{R}^{\cdot} \stackrel{\mathrm{M}}{\longrightarrow} \mathrm{P}_{\mathrm{n}}^{\cdot}
$$

Hauptgleichgewicht:

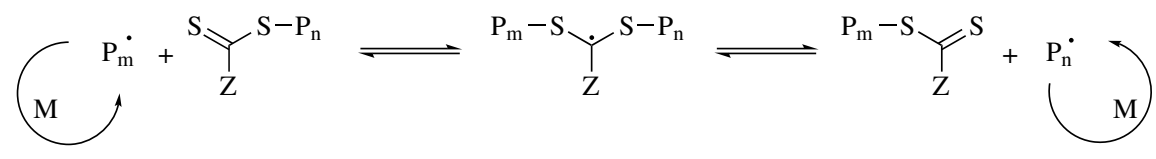

Schema 1: Mechanismus der RAFT-Polymerisation.

Analog zur konventionellen radikalischen Polymerisation wird im ersten Reaktionsschritt der Initiator $I_{2}$ thermisch, photochemisch oder durch Redoxreaktionen in Initiatorradikale $\mathrm{I}^{\bullet}$ gespalten. Diese reagieren mit Monomeren $\mathrm{M}$ und bilden radikalische Polymerketten $\mathrm{P}_{\mathrm{m}}{ }^{\bullet}$. Im RAFT-Vorgleichgewicht addiert die Kette an ein RAFT-Agens und bildet ein intermediäres Radikal. Dieses kann nun die R-Gruppe oder die Polymerkette abspalten. Die Bildung des R-Gruppen-Radikals $\mathrm{R}^{\bullet}$ ist in der Regel thermodynamisch bevorzugt, da sie häufig elektronenziehende Substituenten wie Cyanide oder Carboxylate trägt. Das RGruppen-Radikal kann ebenfalls mit Monomeren reagieren und eine 
wachsende Kette bilden. Alle wachsenden Ketten in Lösung stehen mit den an das RAFT-Agens gebundenen Ketten im Hauptgleichgewicht. Die Propagation verteilt sich somit gleichmäßig über alle Ketten. Das Resultat ist ein Polymer mit einer schmalen Molmassenverteilung. Zudem ist die Molmasse des Polymers proportional zum Umsatz und dieser proportional zur Reaktionzeit. Sie berechnet sich nach

$$
\bar{M}_{\mathrm{n}}=\frac{c_{\mathrm{M}, 0}-c_{\mathrm{M}, t}}{c_{\mathrm{RAFT}}} \cdot M_{\text {Monomer }}+M_{\mathrm{RAFT}} .
$$

Dabei ist $c_{\mathrm{M}, 0}-c_{\mathrm{M}, t}$ die Differenz zwischen Ausgangskonzentration an Monomer und Monomerkonzentration zum Zeitpunkt $t, c_{\mathrm{RAFT}}$ die Konzentration an RAFT-Agens sowie $M_{\text {Monomer }}$ und $M_{\text {RAFT }}$ die molare Massen von Monomer und RAFT-Agens.

\subsubsection{Die Azobenzol-Gruppe als Photoschalter}

Das Azobenzol wurde als einfacher Vertreter der Diimine 1834 von Mitscherlich entdeckt und als Stickstoffbenzid beschrieben. ${ }^{33} 100$ Jahre später wurde bei Untersuchungen zur Lichtempfindlichkeit von Azofarbstoffen die photochemische Aktivität der Azobenzole entdeckt, die für deren Abbau unter Bestrahlung mit Sonnenlicht verantwortlich ist. ${ }^{34}$ Die photochemische Isomerisierung von Azobenzol wurde 1937 bei Löslichkeitsexperimenten entdeckt. Nach Bestrahlung mit Sonnenlicht ließ sich das nun als cis-Isomer vorliegende Azobenzol besser in Aceton lösen, welches mit dem erhöhten Dipolmoment begründet wurde. ${ }^{35}$

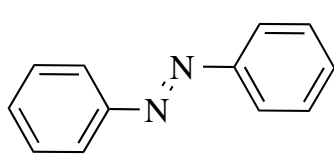

$0 \mathrm{D}$

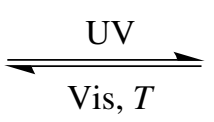

Vis, $T$

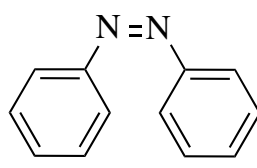

$3 \mathrm{D}$

Schema 2: trans $\leftrightarrow$ cis-Isomerisierung von Azobenzol. 
Azobenzole wurden seitdem als Photoschalter weiter erforscht und finden in unterschiedlichsten Bereichen Anwendung. Photoschaltbare Polymere, ${ }^{36-41}$ molekulare Maschinen, ${ }^{42-45}$ oberflächenmodifizierte Materialien, ${ }^{46-50}$ modifizierte Proteine ${ }^{51-54}$ sowie photoschaltbare HostGuest-Systeme ${ }^{55-58}$ zeigen die vielseitige Einsetzbarkeit von Azobenzolen. Diese Systeme nutzen die Möglichkeit die Azobenzol-Gruppe photochemisch reversibel zwischen trans- und cis-Isomer schalten zu können. Durch Bestrahlung mit UV-Licht $(\lambda \approx 365 \mathrm{~nm})$ kann die trans $\rightarrow$ cis-Isomerisierung induziert werden. Die Isomerisierung zurück zur thermodynamisch günstigeren trans-Konfiguration kann sowohl thermisch als auch durch Bestrahlung mit sichtbarem Licht $(\lambda \approx 430 \mathrm{~nm})$ erfolgen. ${ }^{43,59-62}$ Durch das Einbringen von Substituenten ändern sich die zur Isomerisierung benötigten Wellenlängen aufgrund der Änderung in der elektronischen Struktur. Elektronendonierende Substituenten in ortho-Position führen zu einer Rotverschiebung der $\mathrm{n} \rightarrow \pi^{*}$-Bande im trans-Isomer sowie einer zu Blauverschiebung im cis-Isomer. ${ }^{63}$ Die Verschiebungen sind mit steigender Anzahl und Größe der Substituenten stärker. ${ }^{64}$ Für den $\pi \rightarrow \pi^{*}$-Übergang im trans-Isomer wurde eine Blauverschiebung durch elektronendonierende Substituenten in orthoPosition beobachtet. Elektronenziehende Substitueten verursachen die entgegengesetzten Trends. ${ }^{64}$

Neben dem Dipolmoment ändert sich auch die Geometrie des Azobenzols durch die Isomerisierung deutlich. Das trans-Isomer ist symmetriebedingt unpolar $(\mu=0 \mathrm{D})$, die Phenylringe stehen in einem Winkel von $180^{\circ}$ zueinander. Das gesamte Molekül ist planar. Das cis-Isomer ist hingegen stark polar $(\mu \approx 3 \mathrm{D})$, die Phenylringe weisen einen Winkel von $60^{\circ}$ zueinander auf und liegen nicht länger in einer Ebene. ${ }^{65}$ Durch die Änderung der Molekülgeometrie sind makroskopische Bewegungen möglich.

Die Isomerisierung von Azobenzol kann über unterschiedliche Reaktionspfade stattfinden. Diese sind in Schema 3 dargestellt. Aus dem freien Elektronenpaar eines Stickstoffatomes kann eine $n \rightarrow \pi^{*}$ Anregung erfolgen. Diese führt zu einer Isomerisierung durch Inver- 
sion an diesem Stickstoffatom. ${ }^{66}$ Alternativ kann eine Anregung vom $\pi$ in das $\pi^{*}$-Orbital erfolgen. Die $\pi$-Bindungsordnung der N-N-Bindung sinkt dadurch auf 0. Eine Rotation um die Bindung ist in der Folge möglich. ${ }^{67}$

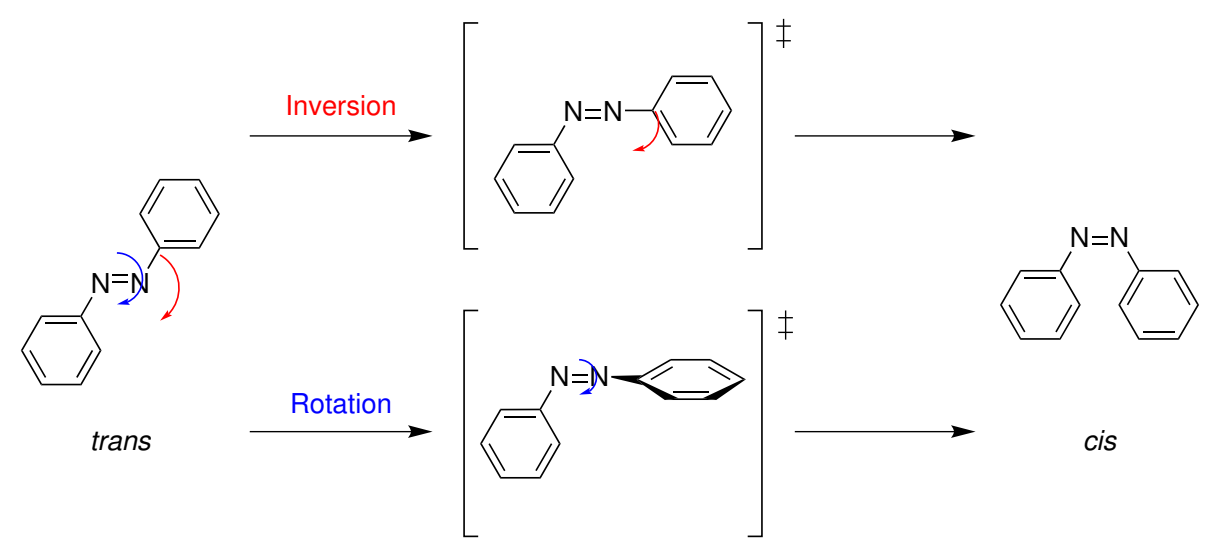

Schema 3: Mögliche Mechanismen der trans $\rightarrow$ cis-Isomerisierung von Azobenzol. $^{43}$ 


\subsection{Synthese der photoschaltbaren Monomere}

Die Synthese der photoschaltbaren Monomere erfolgte ausgehend von 4-Phenylazophenol (AzoOH). Dieses enthält neben der schaltbaren Gruppe auch eine Hydroxy-Gruppe, welche für weitere Syntheseschritte effektiv genutzt werden kann. Als Monomerklasse wurde ein Methacrylat gewählt, da sich radikalische Polymerisation mit Methacrylaten sehr gut durch RAFT-Agenzien kontrollieren lassen. ${ }^{8-12}$ Es ist bekannt, dass eine zu dichte Packung der Azobenzol-Einheiten die Schalteffizienz des trans- zum cis-Isomer verringert. ${ }^{68,69}$ Aus diesem Grund werden alkylische Linker mit verschiedenen Längen zwischen Azobenzol und Methacrylat verwendet. Im ersten Syntheseschritt wurde von 4-Phenylazophenol ausgehend der aliphatische Linker durch eine nukleophile Substitution eingeführt. Abschließend konnten die Zielverbindungen durch Umsetzung mit Methacryloylchlorid erhalten werden.<smiles>Oc1ccc(/N=N/c2ccccc2)cc1</smiles>

AzoOH

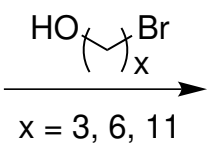<smiles>C=C(C)C(=O)Cl</smiles><smiles>C=C(C)C(=O)Oc1ccc(/N=N/c2ccccc2)cc1</smiles>

AzoMA
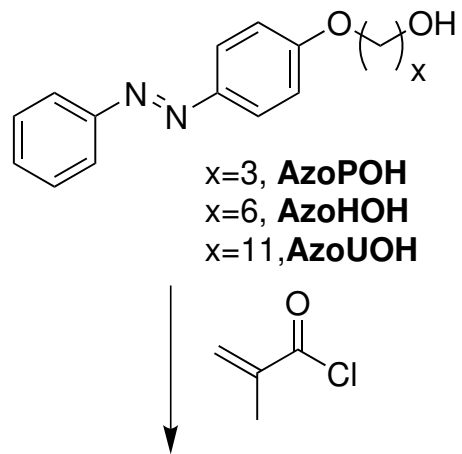

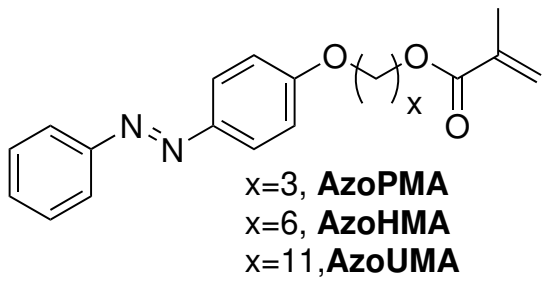

Schema 4: Syntheseweg der photoschaltbaren Monomere AzoMA, AzoPMA, AzoHMA und AzoUMA.

Die Produkte und Zwischenstufen wurden mittels ${ }^{1} \mathrm{H}-\mathrm{NMR}-$ Spektroskopie und ESI-MS untersucht (Siehe Kapitel 6.6). Der 
Schaltvorgang wurde durch UV/Vis-Spektroskopie genauer untersucht. Die Spektren sind in Abbildung 3 gezeigt.
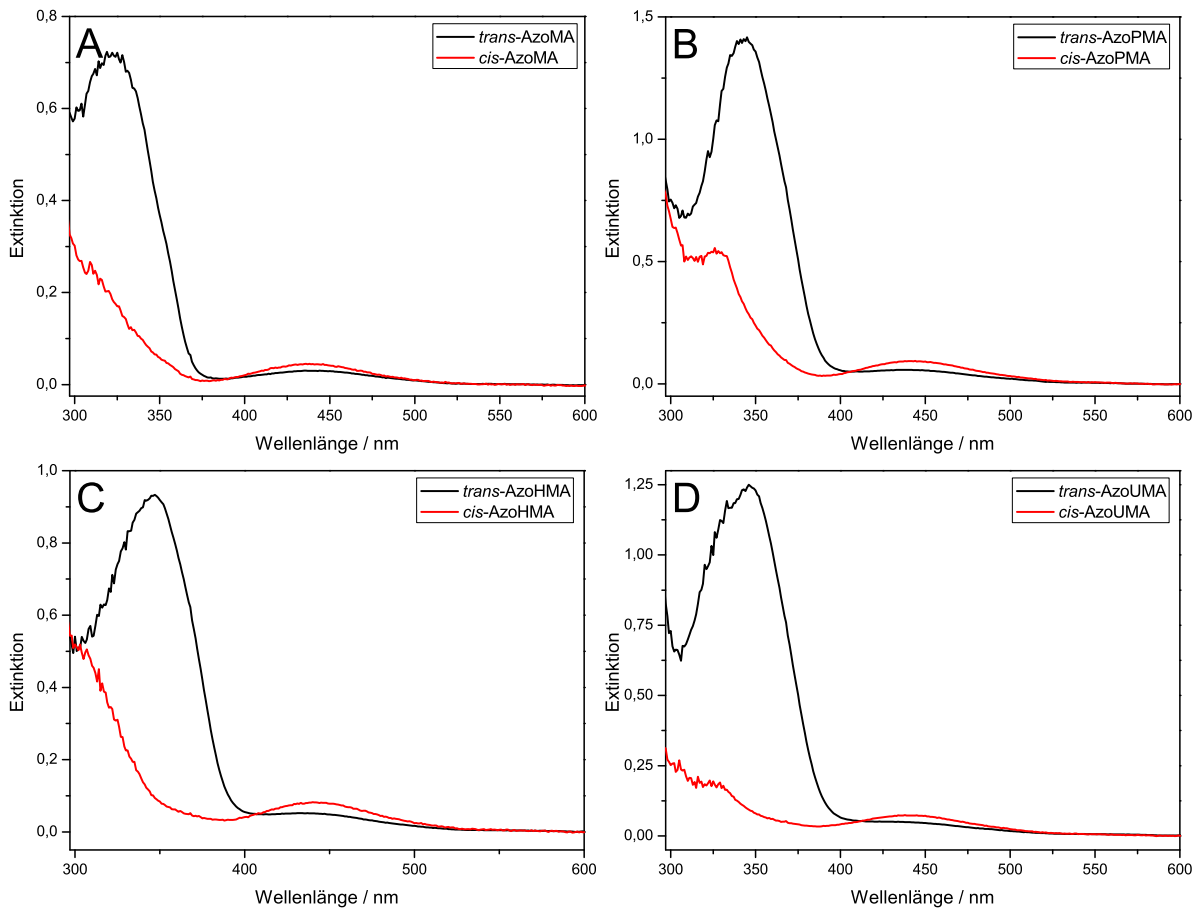

Abbildung 3: UV/Vis-Spektren der photoschaltbaren Monomere AzoMA (A), AzoPMA (B), AzoHMA (C) und AzoUMA (D), in trans(schwarz) und cis-Konfiguration (rot) in THF.

In den Spektren der trans-Isomere sind zwei Absorptionsmaxima zu erkennen. Die Maxima bei etwa $340 \mathrm{~nm}$ sind einem $\pi \rightarrow \pi^{*}$-Übergang zuzuordnen. Zudem ist ein $\mathrm{n} \rightarrow \pi^{*}$-Übergang der Bande bei $440 \mathrm{~nm}$ zuzuordnen. Dieser ist von geringer Intensität, da es sich um einen verbotenen Übergang handelt. In den Spektren der cis-Isomere ist die Bande des $\mathrm{n} \rightarrow \pi^{*}$-Überganges bei $440 \mathrm{~nm}$ deutlich zu erkennen, da es sich im cis-Isomer um einen erlaubten Übergang handelt. Da sich die Wellenlänge der Bande nicht ändert, ist der Übergang sehr gut geeignet um den zeitlichen Verlauf des Schaltprozesses zu untersuchen. 


\subsection{Thermische Relaxationskinetik von photoschaltbaren Monomeren und Polymeren}

Azobenzol-basierte Monomere und Polymere können durch Bestrahlung mit UV-Licht eine trans-cis-Isomerisierung durchlaufen. Um Messungen am cis-Isomer durchzuführen ist es essenziell zu wissen, wie die schnell die thermische Umwandlung des cis-Isomer in das transIsomer abläuft. Dieser Prozess kann durch UV/Vis-Spektroskopie verfolgt werden.

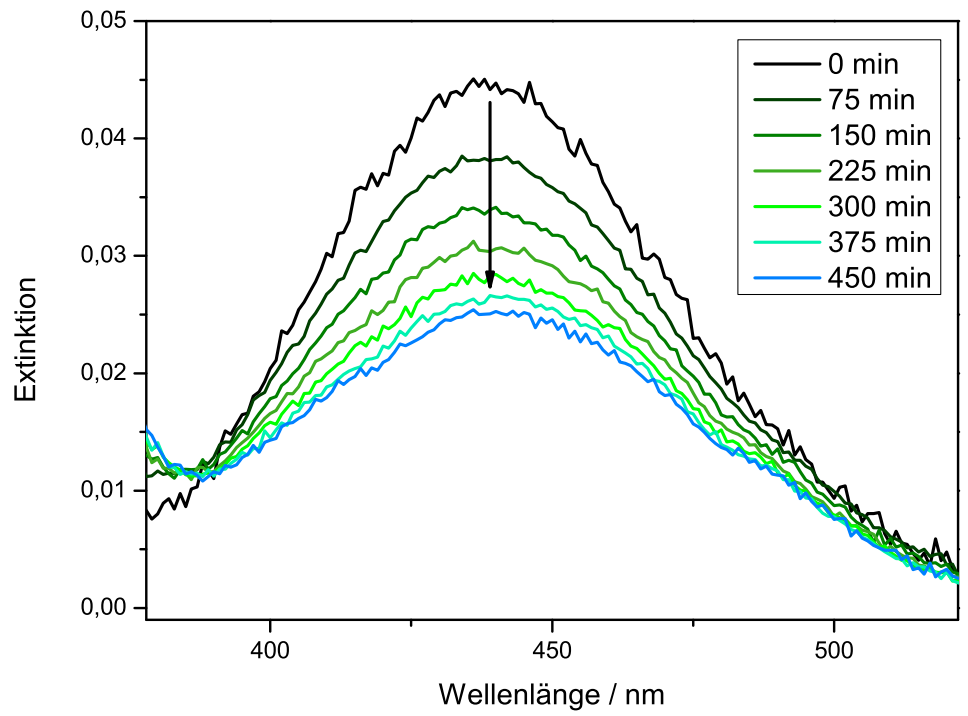

Abbildung 4: Ausschnitt der $\mathrm{n} \rightarrow \pi^{*}$-Bande aus den zeitaufgelösten UV/VisSpektren von AzoMA in THF bei $60{ }^{\circ} \mathrm{C}$. Die Abnahme der Extinktion wird durch einen Pfeil verdeutlicht.

Wird die Bande des $\mathrm{n} \rightarrow \pi^{*}$-Überganges betrachtet, so setzt sich die Extinktion $E_{\text {ges }}$ aus den Extinktionen der beiden Isomere $E_{\text {cis }}$ und $E_{\text {trans }}$ zusammen.

$$
E_{\text {ges }}=E_{\text {cis }}+E_{\text {trans }}
$$


Die Extinktion setzt sich dabei nach dem Lambert-Beer'schen-Gesetz aus der Konzentration der Probe $c$, der Schichtdicke $d$ sowie des Extinktionskoeffizienten $\varepsilon$.

$$
E=\varepsilon \cdot c \cdot d
$$

Werden nun Gleichung 2 und Gleichung 3 kombiniert, die Schichtdicke $d=1 \mathrm{~cm}$ in den Extinktionskoeffizienten integriert und umgestellt (Herleitung in Kapitel 7.1), so wird folgender Ausdruck für $c_{\mathrm{cis}}$ erhalten

$$
c_{\mathrm{cis}}=\frac{c_{\mathrm{ges}}-\frac{E_{\mathrm{ges}}}{\varepsilon_{\mathrm{trans}}}}{1-\frac{\varepsilon_{\mathrm{cis}}}{\varepsilon_{\mathrm{trans}}}} .
$$

Die Umwandlung des cis- in das trans-Isomer kann als unimolekulare Reaktion erster Ordnung betrachtet werden. Für eine solche Reaktion gilt für $c_{c i s}$ :

$$
c_{\mathrm{cis}}=c_{\mathrm{cis}, 0} \cdot e^{-k \cdot t}
$$

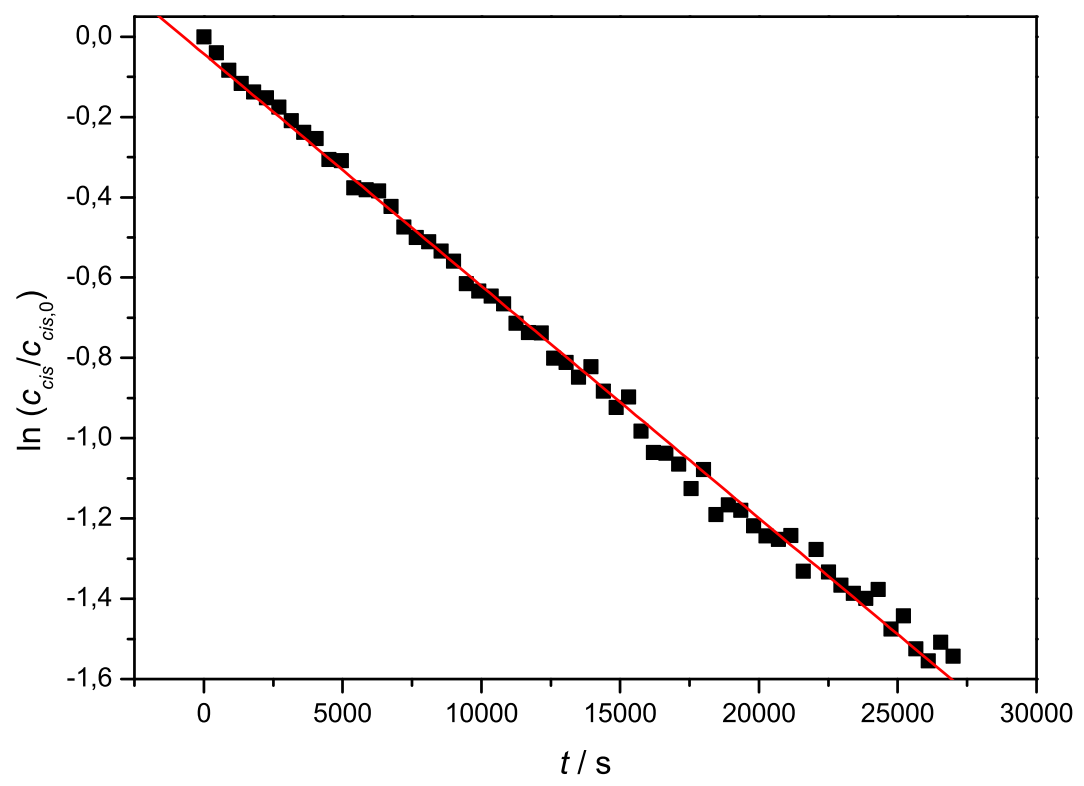

Abbildung 5: Auftragung von $\ln \left(\frac{c_{\mathrm{cis}}}{c_{\mathrm{cis}, 0}}\right)$ gegen $t$ für AzoMA in THF bei $60{ }^{\circ} \mathrm{C}$. 
mit der Ausgangskonzentration des cis-Isomers $c_{\mathrm{cis}, 0}$, dem Geschwindigkeitskoeffizienten $k$ und der Zeit $t$. Der Geschwindigkeitskoeffizient kann nach

$$
\ln \left(\frac{c_{\mathrm{cis}}}{c_{\mathrm{cis}, 0}}\right)=-k \cdot t
$$

durch eine Auftragung von $\ln \left(\frac{c_{\mathrm{cis}}}{c_{\mathrm{cis}, 0}}\right)$ gegen $t$ erhalten werden.

\subsubsection{Bestimmung des Geschwindigkeitskoeffizienten der thermischen Isomerisierung von AzoMA in Abhängigkeit des Lösungsmittels}

Um den Einfluss des Lösungsmittels auf die cis $\rightarrow$ trans-Isomerisierung zu untersuchen, wurde der einfachste Vertreter der photoschaltbaren Monomere, AzoMA untersucht. Es wurden Hexan (0,00 D), Toluol $(0,38$ D), THF (1,63 D) und DMF (3,82 D) gewählt, um sowohl unpolare, als auch mäßig polare sowie stark polare Lösungsmittel abzudecken. Die Messungen wurden an $50 \mu \mathrm{M}$-Lösungen des Monomers in den genannten Lösungsmitteln durchgeführt. Vor der Messung wurde die Probe durch Bestrahlung mit UV-Licht für mindestens 30 Minuten eine Isomerisierung in die cis-Konfiguration induziert. Bei 5 verschiedenen Temperaturen wurde die Abnahme der Extinktion der $n \rightarrow \pi^{*}$-Bande zeitaufgelöst gemessen und wie in Kapitel 2.3 beschrieben ausgewertet. Die Ergebnisse sind in Tabelle 1 und Abbildung 6 zusammengefasst.

Tabelle 1: Geschwindigkeitskoeffizienten $k$ der thermischen Isomerisierung von AzoMA in verschiedenen Lösungsmitteln in Abhängigkeit der Temperatur.

\begin{tabular}{lccccc}
\hline$k / 10^{-6} \mathrm{~s}^{-1}$ & $36{ }^{\circ} \mathrm{C}$ & $42{ }^{\circ} \mathrm{C}$ & $48{ }^{\circ} \mathrm{C}$ & $54{ }^{\circ} \mathrm{C}$ & $60{ }^{\circ} \mathrm{C}$ \\
\hline Hexan & $4,5 \pm 0,2$ & $6,3 \pm 0,1$ & $9,9 \pm 0,4$ & $17,4 \pm 0,4$ & $47,8 \pm 0,7$ \\
Toluol & $7,0 \pm 0,9$ & $8,1 \pm 0,1$ & $16,7 \pm 1,1$ & $26,4 \pm 0,5$ & $49,3 \pm 0,9$ \\
THF & $3,05 \pm 0,09$ & $7,7 \pm 0,1$ & $15,5 \pm 0,2$ & $28,4 \pm 0,2$ & $57,8 \pm 0,5$ \\
DMF & $1,5 \pm 0,3$ & $2,8 \pm 0,3$ & $6,2 \pm 0,3$ & $9,3 \pm 0,7$ & $14,8 \pm 0,6$ \\
\hline
\end{tabular}




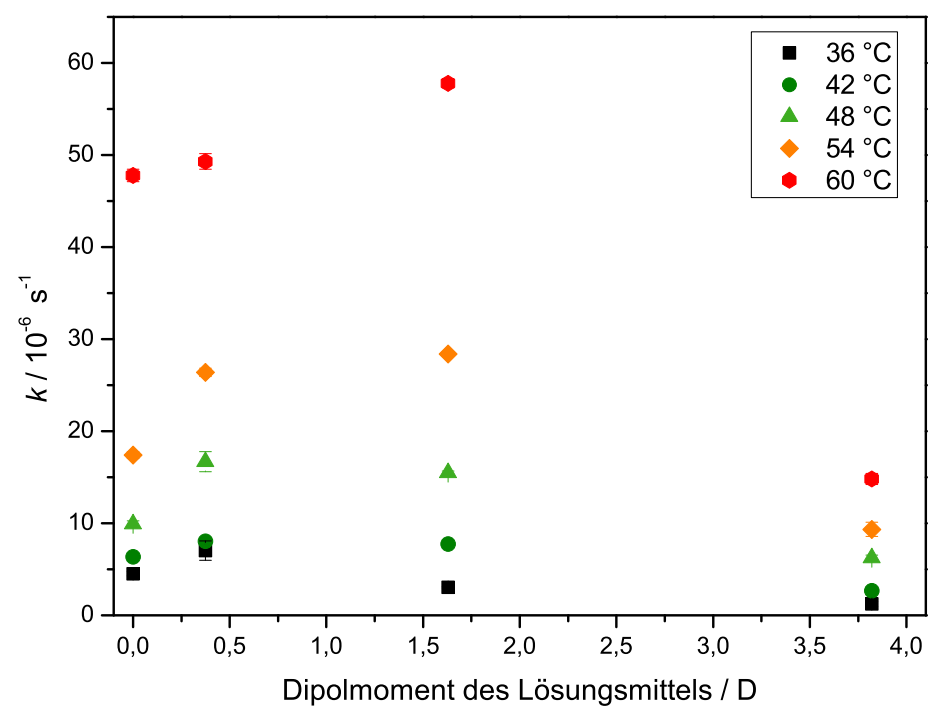

Abbildung 6: Auftragung der Geschwindigkeitskoeffizienten der thermischen Isomerisierung von AzoMA gegen das Dipolmoment des Lösungsmittels bei verschiedenen Temperaturen.

Der Zusammenhang zwischen den Geschwindigkeitskoeffizienten $k$, der Temperatur $T$ und der Aktivierungsenergie der Reaktion $E_{\mathrm{A}}$ kann durch Gleichung 7, der Arrhenius-Gleichung beschrieben werden. Dabei ist $A$ der präexponentielle Arrhenius-Faktor und $R$ die universelle Gaskonstante.

$$
\begin{aligned}
k & =A \cdot \mathrm{e}^{\left(\frac{-E_{\mathrm{A}}}{R \cdot T}\right)} \\
\ln (k) & =\ln (A)-\frac{E_{\mathrm{A}}}{R \cdot T}
\end{aligned}
$$

Durch eine Auftragung der logarithmierten Geschwindigkeitskoeffizienten gegen die reziproke Temperatur kann nach Gleichung 8 die Aktivierungsenergie als Steigung erhalten werden. Diese Auftragung ist in 
Abbildung 7 dargestellt und die Ergebnisse in Tabelle 2 zusammengefasst.

Tabelle 2: Durch Arrhenius-Auftragung bestimmte Aktivierungsenergien $E_{\mathrm{A}}$ für die Isomerisierung von cis-AzoMA zu trans-AzoMA in verschiedenen Lösungsmitteln.

\begin{tabular}{ccccc}
\hline & Hexan & Toluol & THF & DMF \\
\hline$E_{\mathrm{A}} / \mathrm{kJ} \cdot \mathrm{mol}^{-1}$ & $83 \pm 8$ & $72 \pm 8$ & $75 \pm 7$ & $62 \pm 15$ \\
\hline
\end{tabular}

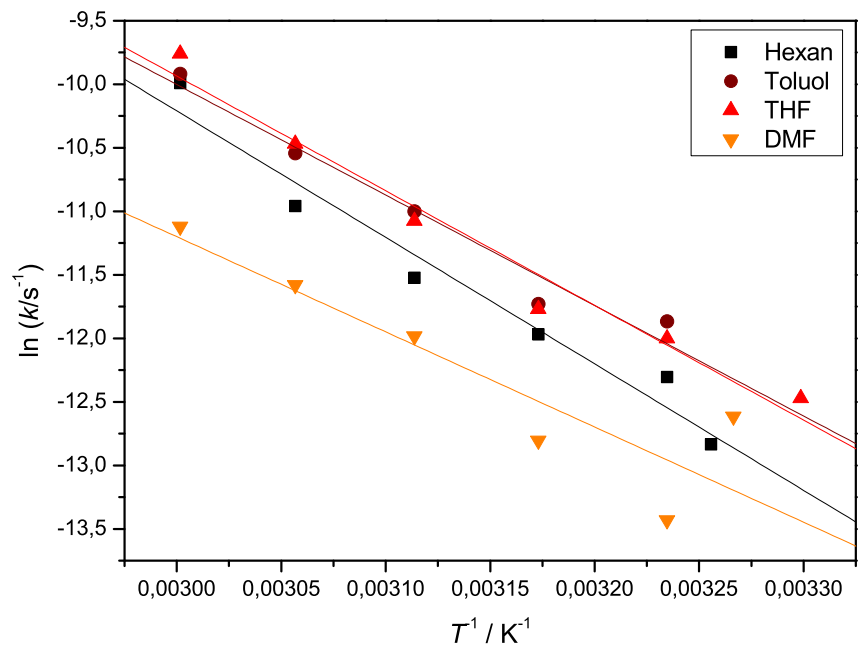

Abbildung 7: Auftragung der logarithmierten Geschwindigkeitskoeffizienten der Isomerisierung gegen die reziproke Temperatur für AzoMA in verschiedenen Lösungsmitteln.

Die Aktivierungenergien $E_{\mathrm{A}}$ des Isomerisierungsprozesses für AzoMA sind in allen verwendeten Lösungsmitteln geringer als für reines Azobenzol mit $95 \mathrm{~kJ} \cdot \mathrm{mol}^{-1}{ }^{59}$ Dies deckt sich mit theoretischen Berechnungen, die eine Verringerung der Aktivierungsenergie mit der Anzahl und Größe von Substituenten am Azobenzol vorhersagen. ${ }^{70}$ Zudem ist zu erkennen, dass die Aktivierungsenergie des Isomerisierungsprozesses mit zunehmender Polarität des Lösungsmittels zunimmt. Der Übergangszustand der Isomerisierung ist sehr stark polar, das Dipolmoment 
ist etwa doppelt so hoch wie das des cis-Isomers. ${ }^{70}$ Die Stabilisierung ist in polaren Lösungsmitteln daher besonders gut und resultiert in einer Verringerung der Aktivierungsenergie der Isomerisierungsreaktion. In unpolaren Lösungsmitteln zeigt sich der konträre Trend, sodass die Aktivierungsenergie hier höher ist. Die Stabilisierung bzw. Destabilisierung von Produkt und Edukt hat nur einen untergeordneten Einfluss.

\subsubsection{Synthese von photoschaltbaren Polymeren}

Aus den photoschaltbaren Monomeren AzoMA, AzoPMA, AzoHMA und AzoUMA wurden durch RAFT-Polymerisation Polymere hergestellt. Dabei wurde 4-Cyano-4-[(dodecylsulfanylthiocarbonyl)sulfanyl]pentansäure (CDSPA) als RAFT-Agens genutzt, die sich gut zur Polymerisation von Methacrylaten eignet. ${ }^{8} 9$ Die Synthese erfolgte in Lösung, N,N-Dimethylformamid (DMF) wurde als Lösungsmittel, 1,1'Azobis(cyclohexancarbonitril) (ACCN) als thermischer Initiator eingesetzt. Die Polymerisation erfolgte bei $90{ }^{\circ} \mathrm{C}$. Die Polymere wurden in Methanol gefällt und durch Zentrifugation abgetrennt. Die genauen Synthesevorschriften sind in Kapitel 6.7 aufgeführt. Die Polymere wurden durch Gelpermeationschromatografie charakterisiert. Alle Polymere zeigten eine schmale, monomodale Molmassenverteilung. Die erhaltenen Größen sind in Tabelle 3 zusammengefasst.

Tabelle 3: Mittlere Molmassen $M_{\mathrm{n}}$ und Dispersitäten $Ð$ der photoschaltbaren Polymere.

\begin{tabular}{lcc}
\hline Polymer & $M_{\mathrm{n}} /\left(\mathrm{g} \cdot \mathrm{mol}^{-1}\right)$ & $Ð$ \\
\hline PAzoMA & 11.700 & 1,37 \\
PAzoPMA & 10.600 & 1,43 \\
PAzoHMA & 5.300 & 1,25 \\
PAzoUMA & 8.700 & 1,27 \\
\hline
\end{tabular}

Analog zu den Spektren der Monomere sind in den Spektren der trans-Isomere zwei Absorptionsmaxima zu erkennen, die einem $\pi \rightarrow \pi^{*}$ Übergang und einem $\mathrm{n} \rightarrow \pi^{*}$-Übergang zuzuordnen sind. In den Spek- 
tren der cis-Isomere ist die Bande des $\mathrm{n} \rightarrow \pi^{*}$-Überganges bei $440 \mathrm{~nm}$ deutlich zu erkennen. Die Positionen der Banden sind im Vergleich zu den entsprechenden Monomeren nicht signifikant verschoben. Die Schaltkinetik der Azobenzolgruppe kann somit auch im Polymer gut anhand der Bande des $n \rightarrow \pi^{*}$-Überganges untersucht werden.
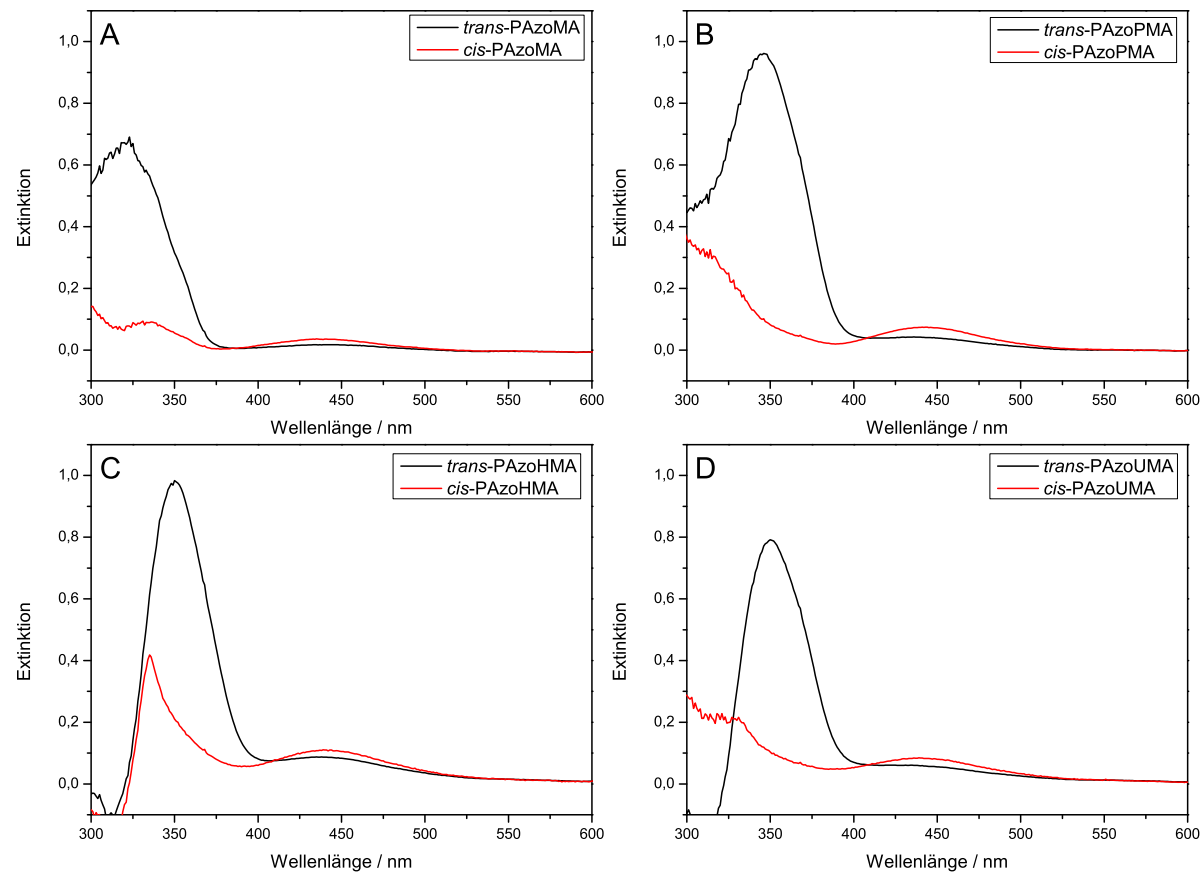

Abbildung 8: UV/Vis-Spektren der photoschaltbaren Polymere PAzoMA (A), PAzoPMA (B), PAzoHMA (C) und PAzoUMA (D), in trans(schwarz) und cis-Konfiguration (rot) in THF.

\subsubsection{Bestimmung des Geschwindigkeitskoeffizienten der thermischen Isomerisierung von Azobenzol-haltigen Monomeren und Polymeren in Abhängigkeit der Länge des verwendeten Linkers}

Um den Einfluss der Länge des verwendeten Linkers auf die Schaltkinetik der photoschaltbaren Mono- und Polymere in Lösung zu untersuchen wurde bei 5 verschiedenen Temperaturen die Abnahme der Extinktion der $n \rightarrow \pi^{*}$-Bande zeitaufgelöst gemessen. Die erhaltenen 
Spektren wurden wie in Kapitel 2.3 beschrieben ausgewertet. Als Lösungsmittel wurde THF gewählt, da alle Proben eine gute Löslichkeit darin aufwiesen.

Die Ergebnisse sind in Tabelle 4 zusammengefasst und in Abbildung 9 grafisch dargestellt. Im weiteren Verlauf des Kapitels wird der Einfluss der Temperatur, der Linkerlänge, sowie der Unterschied zwischen Mono- und Polymer untersucht und diskutiert.

Tabelle 4: Geschwindigkeitskoeffizienten $k$ der thermischen Isomerisierung von verschiedenen photoschaltbaren Mono- und Polymeren in THF in Abhängigkeit von der Temperatur.

\begin{tabular}{lccccc}
\hline$k / 10^{-6} \mathrm{~s}^{-1}$ & $36{ }^{\circ} \mathrm{C}$ & $42{ }^{\circ} \mathrm{C}$ & $48{ }^{\circ} \mathrm{C}$ & $54{ }^{\circ} \mathrm{C}$ & $60{ }^{\circ} \mathrm{C}$ \\
\hline AzoMA & $3,05 \pm 0,09$ & $7,7 \pm 0,2$ & $15,5 \pm 0,2$ & $28,4 \pm 0,2$ & $57,8 \pm 0,5$ \\
AzoPMA & $4,0 \pm 0,6$ & $8,6 \pm 0,2$ & $13,5 \pm 0,6$ & $34,0 \pm 0,2$ & $61 \pm 1$ \\
AzoHMA & $4,9 \pm 0,1$ & $9,6 \pm 0,2$ & $17,9 \pm 0,2$ & $37,1 \pm 0,4$ & $68 \pm 2$ \\
AzoUMA & $5,64 \pm 0,02$ & $10,8 \pm 0,3$ & $18,6 \pm 0,6$ & $38 \pm 1$ & $84 \pm 2$ \\
\hline PAzoMA & $3,8 \pm 0,2$ & $8,1 \pm 0,4$ & $16,3 \pm 0,7$ & $18 \pm 1$ & $49 \pm 3$ \\
PAzoPMA & $6,33 \pm 0,06$ & $12,8 \pm 0,1$ & $20,8 \pm 0,3$ & $40,0 \pm 0,1$ & $83 \pm 1$ \\
PAzoHMA & $7,9 \pm 0,1$ & $13,2 \pm 0,1$ & $22,7 \pm 0,2$ & $41,0 \pm 0,1$ & $89 \pm 1$ \\
PAzoUMA & $7,6 \pm 0,5$ & $10,9 \pm 0,3$ & $21,7 \pm 0,5$ & $46,1 \pm 0,2$ & $95 \pm 3$ \\
\hline
\end{tabular}

Wird die Veränderung der Geschwindigkeitskoeffizienten mit der Temperatur betrachtet, so ist erkennbar, dass die Geschwindigkeit der Isomerisierungsreaktion mit steigender Temperatur für alle untersuchten Mono- und Polymere streng monoton steigt. Im Sinne einer ArrheniusAuftragung (Abb. 10) wurden die logarithmierten Geschwindigkeiten gegen die reziproke Temperatur aufgetragen. Es ist erkennbar, dass alle Messreihen die zu erwartende, lineare Abhängigkeit aufweisen. Aus einer linearen Auftragung wurden zudem die Aktivierungsenergien der Isomerisierungen bestimmt. Sie sind in Tabelle 5 zusammengefasst. 

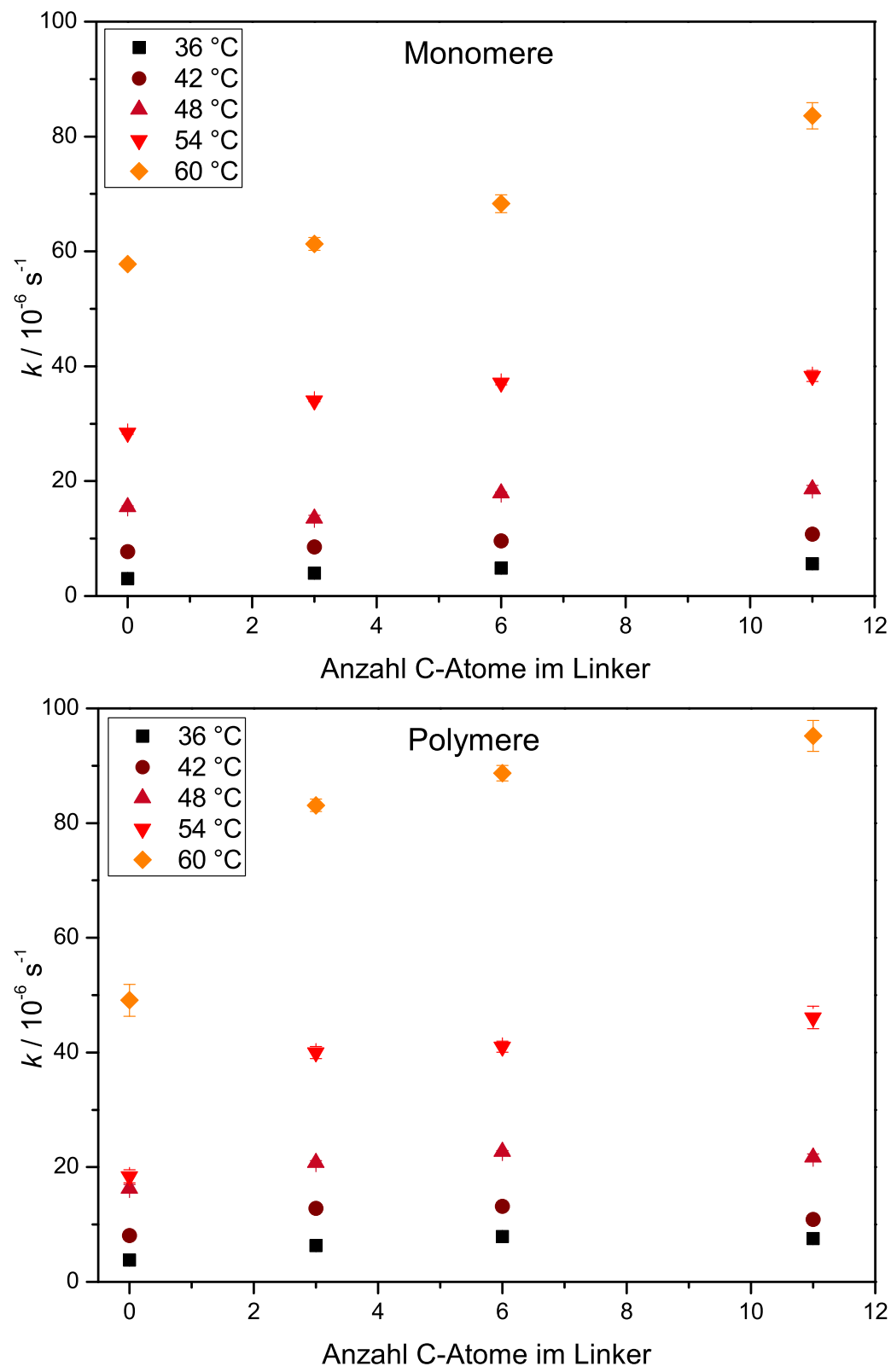

Abbildung 9: Auftragung der Geschwindigkeitskoeffizienten der Isomerisierung gegen die Anzahl der Kohlenstoffatome im verwendeten Linker für photoschaltbare Monomere (oben) und photoschaltbare Polymere (unten) bei verschiedenen Temperaturen. 
Photoschaltbare Mono- und Polymere
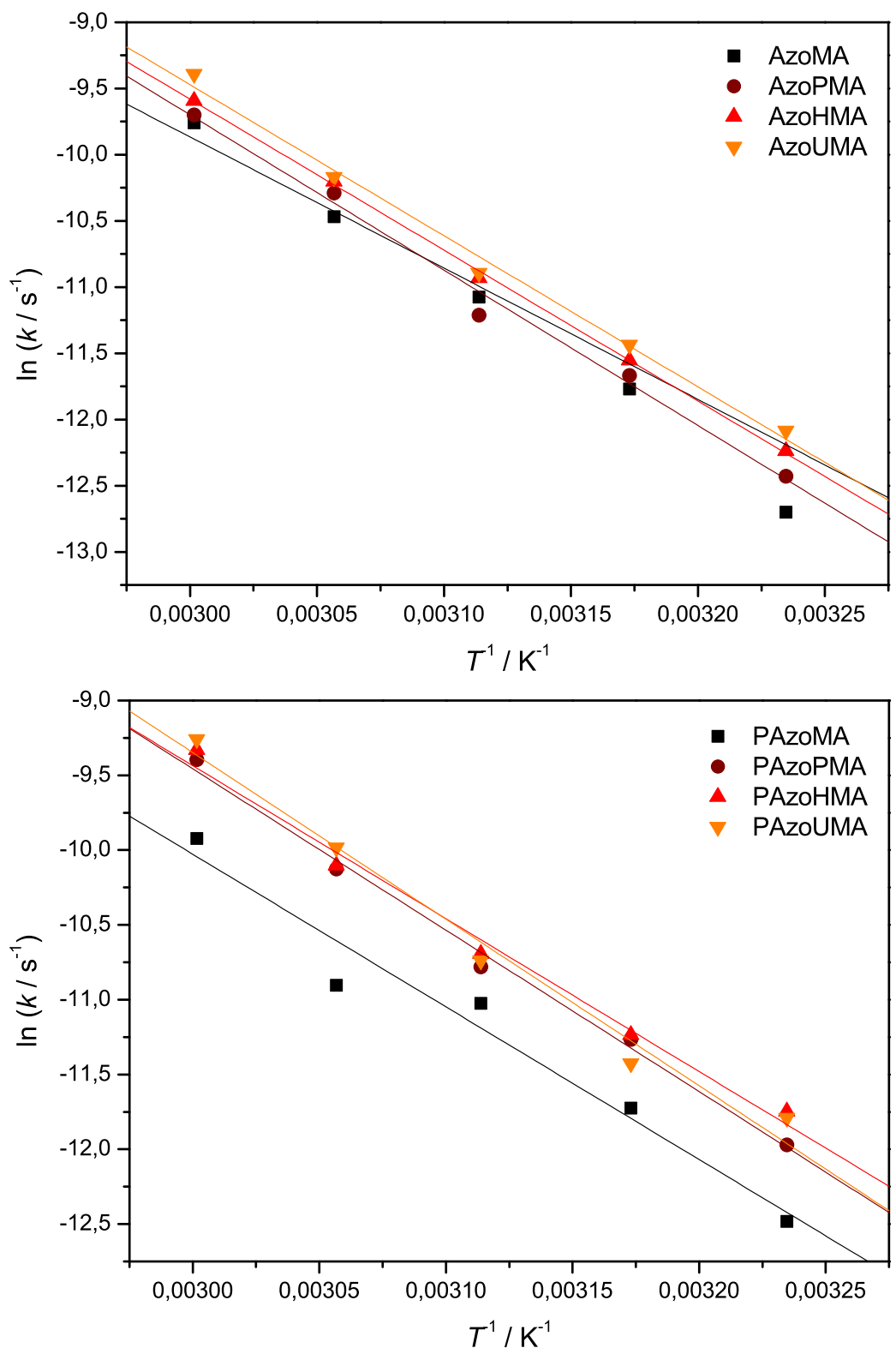

Abbildung 10: Auftragung der logarithmierten Geschwindigkeitskoeffizienten der Isomerisierung gegen die reziproke Temperatur für photoschaltbare Monomere (oben) und photoschaltbare Polymere (unten) mit verschiedenen Längen des verwendeten Linkers. 
Tabelle 5: Aktivierungsenergien $E_{\mathrm{A}}$ in $\mathrm{kJ} \cdot \mathrm{mol}^{-1}$ für die thermischen Isomerisierung von verschiedenen photoschaltbaren Mono- und Polymeren in THF in Abhängigkeit von der Anzahl Kohlenstoffatome im verwendeten Linker.

\begin{tabular}{lcc}
\hline Linkerlänge & Monomer & Polymer \\
\hline 0 & $97 \pm 3$ & $85 \pm 9$ \\
3 & $98 \pm 5$ & $90 \pm 4$ \\
6 & $95 \pm 2$ & $85 \pm 5$ \\
11 & $95 \pm 5$ & $93 \pm 7$ \\
\hline
\end{tabular}

Ein Vergleich der Aktivierungsenergien der Monomere zeigt, dass die Aktivierungsenergien keine Abhängigkeit vom verwendeten Linker zeigen, sondern die Werte im Rahmen des Fehlerbereiches übereinstimmen. Dies deckt sich mit theoretischen Berechnungen zu substituierten Azobenzolen. Dabei wurde eine Verringerung der Aktivierungsenergie mit der Anzahl und der Größe der Substituenten vorausgesagt. ${ }^{70}$ Die Aktivierungsenergie ist hier ähnlich zu der von reinem Azobenzol $\left(95 \mathrm{~kJ} \cdot \mathrm{mol}^{-1}\right),{ }^{59}$ sodass ein einzelner Substituent am Azobenzol noch keine signifikante Auswirkung auf die Aktivierungsenergie zeigt. Auch die Länge des Linkers übt hier keinen Einfluss aus. Der sterische Anspruch eines Substituenten ist maßgeblich dafür, dass die Aktivierungsenergie des Isomerisierungsprozesses verringert wird. ${ }^{59}$ Eine reine Verlängerung eines linearen Substituenten scheint daher den sterischen Anspruch nicht derartig zu erhöhen, sodass die Aktivierungsenergie sinkt.

Die Aktivierungsenergien der zugehörigen Polymere zeigen einen analogen Trend. Es ist keine signifikante Abhängigkeit der Aktivierungsenergie von der Linkerlänge zu erkennen. Lediglich die Aktivierungsenergie für PAzoUMA, das Polymer mit dem längsten Linker, zeigt eine höhere Aktivierungsenergie als die anderen untersuchten Polymere. Alle Aktivierungsenergien sind geringer, als die des zugehörigen Monomers. Durch die Polymerisation werden die Azobenzole am Ende des Linkers verknüpft, sodass sie sich in räumlicher Nähe befinden. Der 
sterische Anspruch des Substituenten am Azobenzol ist durch die Polymerisation der Linker erhöht. Daraus resultiert die geringere Aktivierungsenergie im Vergleich zum Monomer. Im Fall des PAzoUMA enthält der Linker eine Undecyl-Kette, wodurch der Abstand des Azobenzols zum Polymerrückgrat im Vergleich zu den anderen Polymeren höher ist. Dadurch sinkt der sterische Anspruch der Substituenten. Folglich ist die Aktivierungsenergie im Vergleich zu den anderen Polymeren höher.

Ein Vergleich der Geschwindigkeitskoeffizienten gegen die Temperatur zwischen Monomer und zugehörigem Polymer ist in Abb. 11 gezeigt. Wie schon aus den Arrhenius-Auftragungen ersichtlich ist, wird die Reaktion mit steigender Temperatur schneller. Es ist zudem klar erkennbar, dass die Isomerisierung im Polymer schneller abläuft, als im zugehörigen Monomer. Die einzige Ausnahme bildet hier AzoMA/PAzoMA bei hohen Temperaturen. Der allgemeine Trend lässt sich zum einen durch die Aktivierungsenergien erklären, die in den photoschaltbaren Polymeren geringer sind, als in den photoschaltbaren Monomeren. Zum anderen schafft die makromolekulare Struktur in der die Azobenzol-Gruppe integriert ist, eine unpolare Umgebung, in der die Bildung des trans-Isomers bevorzugt ist. Es ist bekannt, dass schaltbare Gruppen in Polymeren derartige kooperative Effekte zeigen. ${ }^{71}$

Um das Verhalten von PAzoMA bei hohen Temperaturen zu verstehen, muss zunächst das thermische Verhalten von Polymerern mit steigender Temperatur betrachtet werden. Als charakteristische Größe um zu beschreiben, wie stark eine Polymerkette geknäult ist, kann der quadrierte mittlere End-zu-End-Abstand $\left\langle R^{2}\right\rangle$ herangezogen werden. Dieser ist proportional zur Persistenzlänge $l_{p}$, welche antiproportional zur Temperatur ist. Daraus folgt, dass mit steigender Temperatur $\left\langle R^{2}\right\rangle$ abnimmt, das Polymer sich also stärker knäult. ${ }^{72,73}$

Durch die zunehmende Verknäulung mit steigender Temperatur erhöht sich auch die sterische Hinderung für die Isomerisierung der Azobenzol-Gruppe. Hinzu kommt noch, dass bei PAzoMA die Azobenzol-Gruppe direkt am Polymerrückgrat gebunden ist, sodass 
die sterische Hinderung nicht durch eine günstige Linkeranordnung reduziert werden kann. Daraus resultiert eine Isomerisierungsgeschwindigkeit, die langsamer ist als beim zugehörigen Monomer.
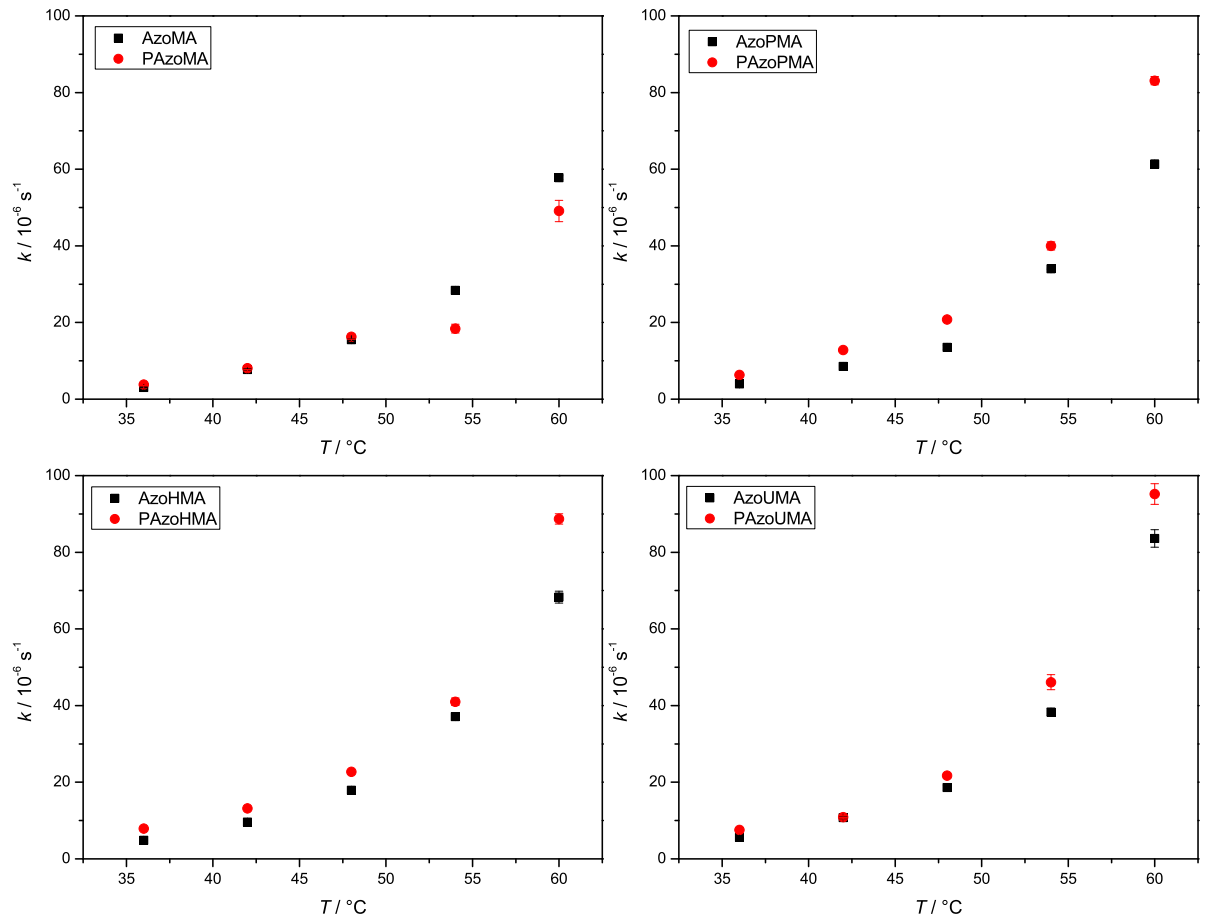

Abbildung 11: Geschwindigkeitskoeffizienten der thermischen Isomerisierung von verschiedenen photoschaltbaren Mono- und Polymeren in THF in Abhängigkeit von der Temperatur, AzoMA und PAzoMA (oben, links), AzoPMA und PAzoPMA (oben rechts), AzoHMA und PAzoHMA (unten links) sowie AzoUMA und PAzoUMA (unten rechts).

Zuletzt wurde die Abhängigkeit der Geschwindigkeitskoeffizienten von der Länge des verwendeten Linkers untersucht. Zur Veranschaulichung wurden für jede Temperatur die Geschwindigkeitskoeffizenten gegen die Linkerlänge aufgetragen und in Abb. 12 dargestellt. 

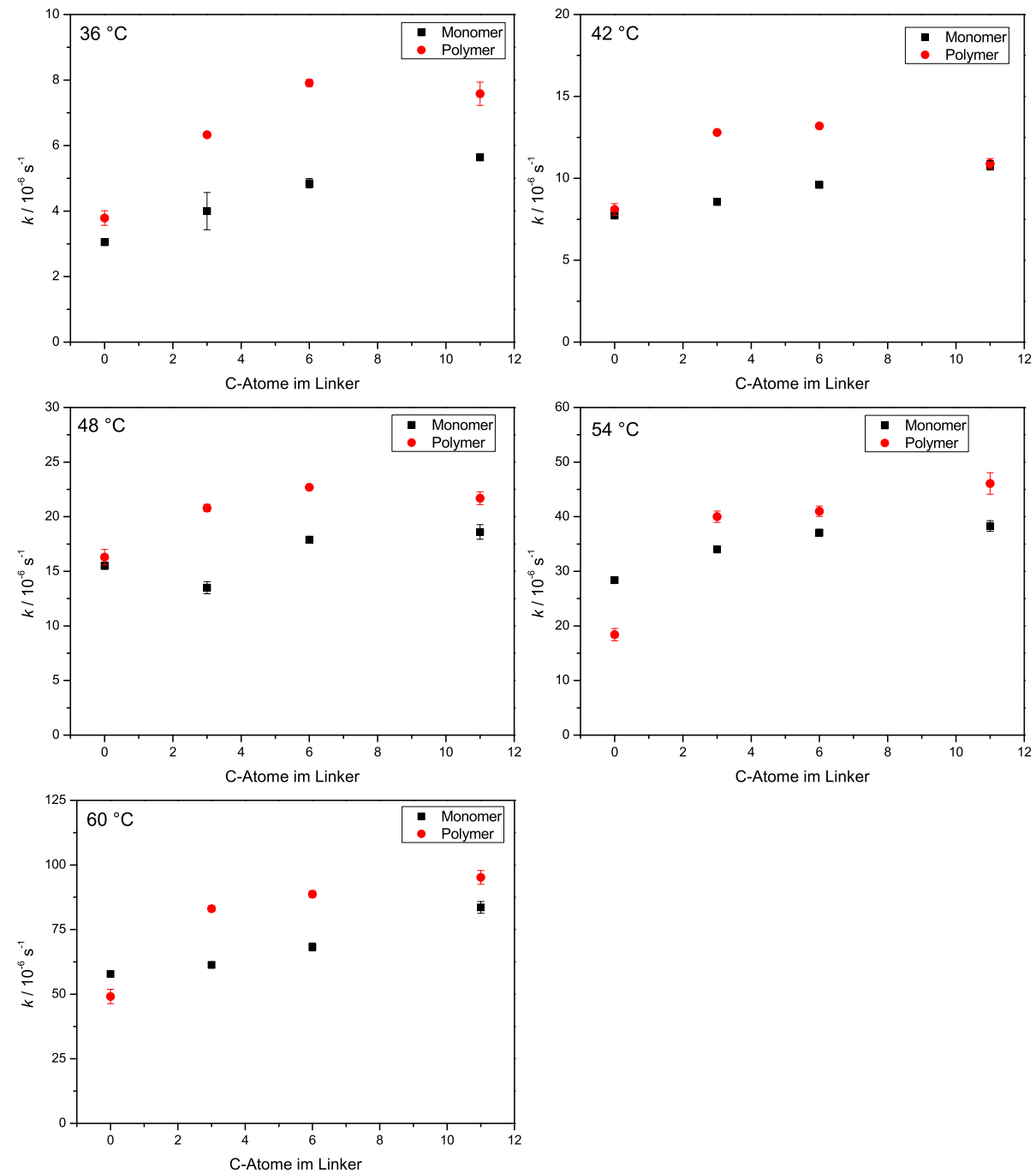

Abbildung 12: Geschwindigkeitskoeffizienten der thermischen Isomerisierung von verschiedenen photoschaltbaren Mono- und Polymeren in THF in Abhängigkeit von der Anzahl an Kohlenstoffatomen im verwendeten Linker bei verschiedenen Temperaturen.

Ein Vergleich der Monomere zeigt, dass bei konstanter Temperatur die Isomerisierungsgeschwindigkeit mit der Linkerlänge streng monoton steigt. Dies ist für alle betrachteten Temperaturen der Fall. Die einzige 
Ausnahme bildet hier der Wert für AzoPMA bei $48{ }^{\circ} \mathrm{C}$, welcher als Ausreißer betrachtet wird. Die Messungen wurden in stark verdünnter Lösung der Monomere durchgeführt, sodass kooperative Effekte zwischen den Monomeren vernachlässigbar sein sollten. Es konnte bereits gezeigt werden, dass der sterische Effekt durch die Größe des Linkers und des Methacrylates keinen Einfluss auf die Aktivierungsenergie des Isomerisierungsprozesses hat. Daher ist anzunehmen, dass auch kein signifikanter Effekt für die Reaktionsgeschwindigkeit vorliegt. Als mögliche Begründung verbleiben elektronische Effekte, die von dem Substituenten des Azobenzols ausgehen. Die hier verwendeten Substituenten ähneln im Falle von AzoUMA, AzoHMA und AzoPMA Alkoxy-Substituenten, welche einen elektronendonierenden Einfluss ausüben. Dieser Effekt ist bei AzoUMA am größten und sinkt über AzoHMA, AzoPMA zu AzoMA ab. Elektronen-donierende Substituenten führen am Azobenzol zu einer größeren Elektronendichte im $\pi^{*}$ Orbital und verringern somit die Bindungsordnung der N-N-Bindung. Eine verringerte N-N-Bindungsordnung vereinfacht die Isomerisierung und führt zu einer Beschleunigung der Reaktion. Dies deckt sich mit den Ergebnissen, bei denen der Geschwindigkeitskoeffizient der Reaktion von AzoMA über AzoPMA, AzoHMA zu AzoUMA steigt.

Im Polymer ist die Isomerisierungsreaktion im Vergleich zum Monomer beschleunigt. Die Ausnahme bildet dabei PAzoMA bei hohen Temperaturen, welche bereits zuvor erklärt wurde. Der Zusammenhang zwischen Geschwindigkeitskoeffizient und Linkerlänge unterscheidet sich von dem der Monomere. Analog zu den Monomeren ist zunächst ein Anstieg der Reaktionsgeschwindigkeit mit der Linkerlänge für alle Temperaturen zu erkennen. Dieser lässt sich zum einen durch die beschriebenen elektronischen Effekte der Substituenten erklären. Zum anderen sinkt mit steigender Linkerlänge der sterische Anspruch der Polymerkette, sodass die Isomerisierungsreaktion schneller ablaufen kann. Bei niedrigen Temperaturen $\left(36-48^{\circ} \mathrm{C}\right)$ sinkt die Reaktionsgeschwindigkeit jedoch für PAzoUMA wieder ab. Durch den langen Linker weisen 
die Azobenzol-Gruppen den größten Abstand der verwendeten Polymere auf. Dadurch wird die Auswirkung des kooperativen Effektes, der die Reaktion beschleunigt, abgeschwächt und die Reaktion läuft im Vergleich zu PAzoHMA langsamer ab. Die Verringerung des kooperativen Effektes ist bei 54 und $60{ }^{\circ} \mathrm{C}$ nicht sichtbar, da die Polymerkette bei hohen Temperaturen, wie bereits beschrieben, stärker geknäult ist und somit die Abstände der Azobenzol-Gruppen klein bleiben. Der kooperative Effekt bleibt daher bestehen. Eine Abschwächung des Effektes bei hohen Temperaturen ist möglicherweise bei einem Polymer mit einem noch längeren Linker zu beobachten.

\subsection{Untersuchung von photoschaltbaren Polymerdünnfilmen}

Zusätzlich zur Charakterisierung der Kinetik photoschaltbarer Monound Polymere in Lösung wurden diese auch in fester Phase untersucht. Dazu wurden Mono- und Polymerlösungen durch Spincoating auf Siliciumwafer aufgebracht. Es zeigte sich, dass für die Monomere trotz Variation des Lösungsmittels und Massenanteil des Monomers kein homogener Film erhalten werden konnte, sondern sich der Feststoff in einige Millimeter große Aggregate zusammenlagerte. Für alle Polymere konnten durch Spincoaten einer Lösung des Polymers in Propylenglycolmonomethyletheracetat (PGMEA) Dünnfilme hergestellt werden. Die Schichtdicke wurde an unterschiedlichen Stellen der Probe durch Ellipsometrie bestimmt um Einheitlichkeit der Filme zu kontrollieren. Die Filme von PAzoMA, PAzoPMA und PAzoHMA wiesen eine homogene Schichtdicke auf, während Filme von PAzoUMA große Ungleichmäßigkeiten zeigten und nicht weiter untersucht wurden. Durch Ellipsometrie wurde die Schichtdicke der Proben in drei Zuständen bestimmt: Im Ausgangszustand $(\alpha)$, nach Bestrahlung mit UV-Licht $(\beta)$ und nach thermischer Relaxation in den Ausgangszustand $(\gamma)$. Zur systematischen Untersuchung wurden für jedes Polymer 5 Proben mit unterschiedlichen Ausgangsschichtdicken angefertigt. Es konnte eine Abnahme der Schichtdicke im bestrahlten Zustand für alle Proben aller 
Polymere beobachtet werden. Die relative Abnahme der Schichtdicke $\Delta h_{\text {rel }}$ kann durch

$$
\Delta h_{\mathrm{rel}}=1-\frac{2 \beta}{\alpha+\gamma}
$$

berechnet werden. Die Ergebnisse sind in Tabelle 6 zusammengestellt und in Abbildung 13 grafisch dargestellt. Um die Reversibilität des Schaltprozesses im Festkörper zu untersuchen wurden am Beispiel von PAzoPMA mehrere Zyklen des Schaltprozesses durchgeführt und die Schichtdicken bestimmt (Abb. 14).

Tabelle 6: Schichtdicke der Polymerfilme vor $(\alpha)$ und nach $(\beta)$ der Bestrahlung mit UV-Licht sowie nach thermischer Relaxation $(\gamma)$, zudem relative Höhendifferenz $\Delta h_{\text {rel }}$ und Mittelwert der relativen Höhendifferenz $\Delta \bar{h}_{\text {rel }}$.

\begin{tabular}{lccccc}
\hline Polymer & $\alpha / \mathrm{nm}$ & $\beta / \mathrm{nm}$ & $\gamma / \mathrm{nm}$ & $\Delta h_{\text {rel }} / \%$ & $\Delta \bar{h}_{\text {rel }} / \%$ \\
\hline & $54,7 \pm 0,3$ & $52,1 \pm 0,2$ & $54,8 \pm 0,3$ & $4,8 \pm 0,5$ & \\
& $123 \pm 1$ & $119,2 \pm 0,2$ & $121,8 \pm 0,3$ & $2,7 \pm 0,6$ & \\
PAzoMA & $193 \pm 4$ & $188,3 \pm 0,4$ & $193,6 \pm 0,3$ & $2,5 \pm 1,0$ & $3,2 \pm 1,0$ \\
& $322 \pm 4$ & $313,7 \pm 0,7$ & $326,0 \pm 0,6$ & $3,2 \pm 0,7$ & \\
& $510 \pm 10$ & $493,5 \pm 0,9$ & $508,5 \pm 1,0$ & $2,6 \pm 1,2$ & \\
\hline & $42,1 \pm 0,2$ & $41,7 \pm 0,2$ & $42,5 \pm 0,2$ & $1,4 \pm 0,6$ & \\
& $95,3 \pm 0,5$ & $92,3 \pm 0,7$ & $95,6 \pm 0,4$ & $3,3 \pm 0,8$ & \\
PAzoPMA & $171,1 \pm 0,6$ & $164,0 \pm 0,8$ & $170,6 \pm 0,6$ & $4,0 \pm 0,5$ & $2,9 \pm 1,0$ \\
& $258,2 \pm 1,1$ & $251,2 \pm 0,7$ & $258,8 \pm 0,4$ & $2,8 \pm 0,4$ & \\
& $317 \pm 2$ & $307,2 \pm 0,6$ & $317,7 \pm 0,8$ & $3,2 \pm 0,3$ & \\
\hline & $46,7 \pm 0,3$ & $43,7 \pm 0,2$ & $44,7 \pm 0,2$ & $4,4 \pm 0,6$ & \\
& $76 \pm 1$ & $72,7 \pm 0,2$ & $76,1 \pm 0,2$ & $4,4 \pm 0,5$ & \\
PAzoHMA & $221 \pm 2$ & $197 \pm 2$ & $215,5 \pm 0,8$ & $9,8 \pm 0,8$ & $7 \pm 2$ \\
& $342,1 \pm 0,8$ & $319 \pm 3$ & $342,6 \pm 1,0$ & $6,9 \pm 0,8$ & \\
& $643 \pm 3$ & $594 \pm 2$ & $644 \pm 4$ & $7,7 \pm 0,4$ & \\
\hline
\end{tabular}



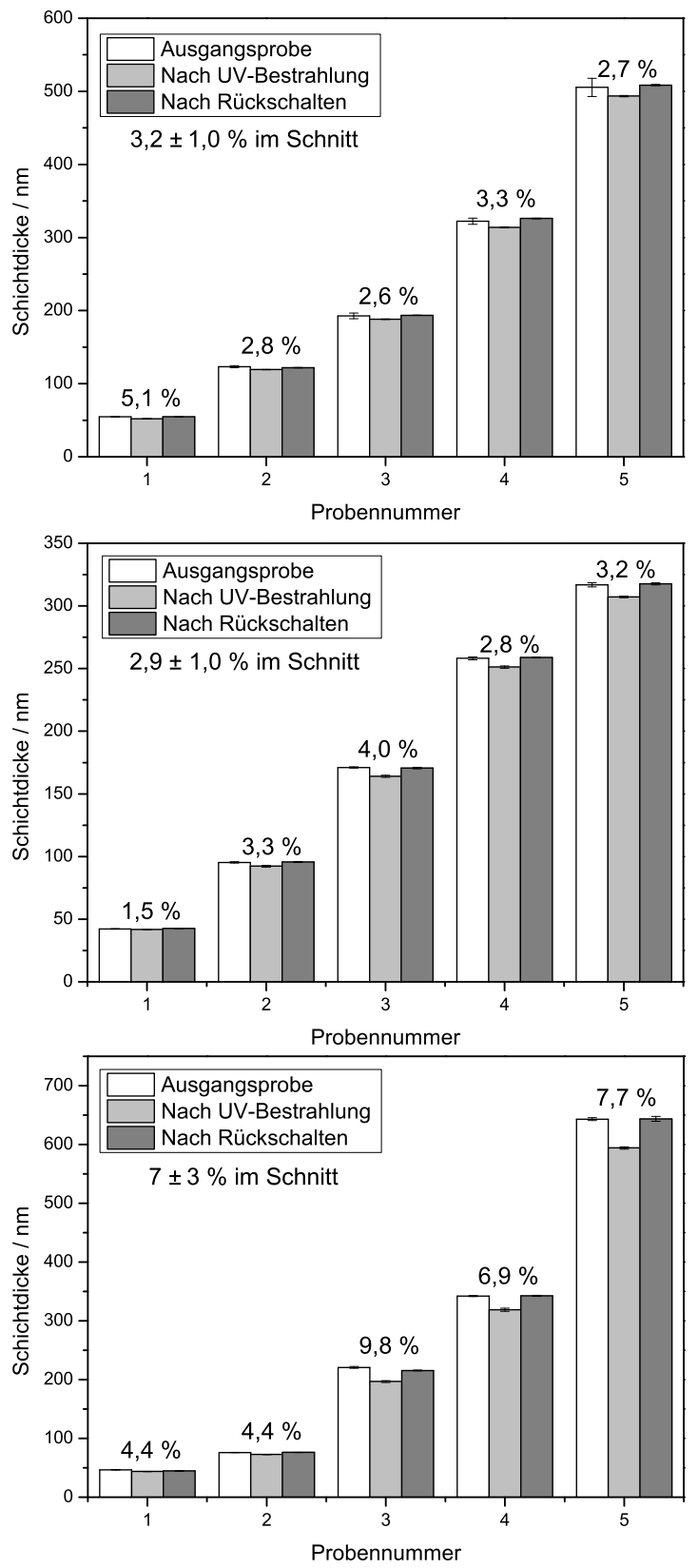

Abbildung 13: Schichtdicken von photoschaltbaren Polymerfilmen aus PAzoMA (Oben), PAzoPMA (Mitte) und PAzoHMA (Unten) im Laufe eines Bestrahlungszyklus. 


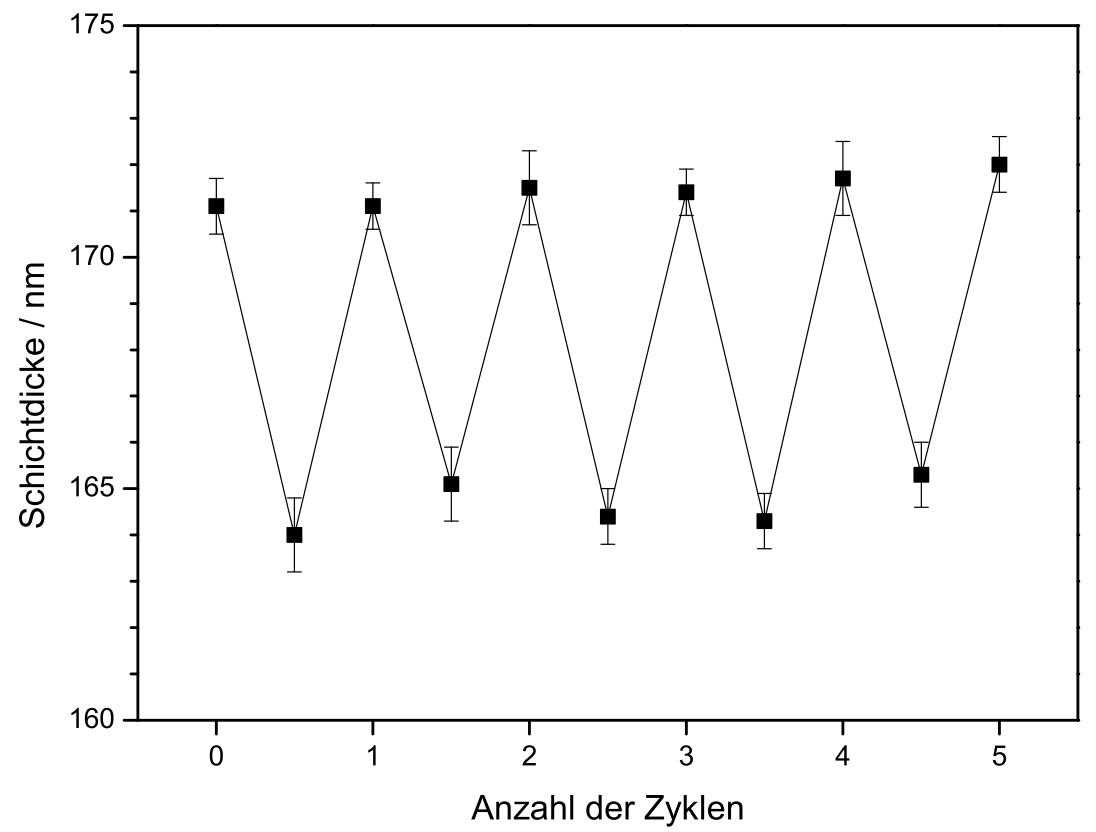

Abbildung 14: Schichtdicke eines PAzoPMA-Dünnfilmes in Abhängigkeit von der Anzahl an Bestrahlungszyklen.

Durch Bestimmung der Schichtdicken in mehreren Bestrahlungszyklen konnte gezeigt werden, dass der Prozess der Schichtdickenänderung reversibel ist. Dies ist typisch für Azobenzol-basierte Systeme, deren Schaltbarkeit sich durch eine hohe Reversibilität auszeichnet und zudem eine große Stabilität des Photoschalters mit bis zu $10^{6}$ Schaltzyklen aufweisen. ${ }^{74}$ Es ist daher anzunehmen, dass die Schichtdickenänderung durch den Isomerisierungsprozess der Azobenzol-Gruppe in den photoschaltbaren Polymeren verursacht wird. Eine Verringerung der Schichtdicke durch einen UV-induzierten Abbauprozess kann ausgeschlossen werden. Die Probenmasse sollte daher konstant bleiben. Die Änderung der Schichtdicke geht daher mit einer Änderung der Dichte einher. Eine Erhöhung der Dichte ist in Polymeren möglich, wenn sich das freie Volumen im Polymer verringert. Dafür können hier zwei Faktoren maßgeblich sein. 
Photoschaltbare Mono- und Polymere

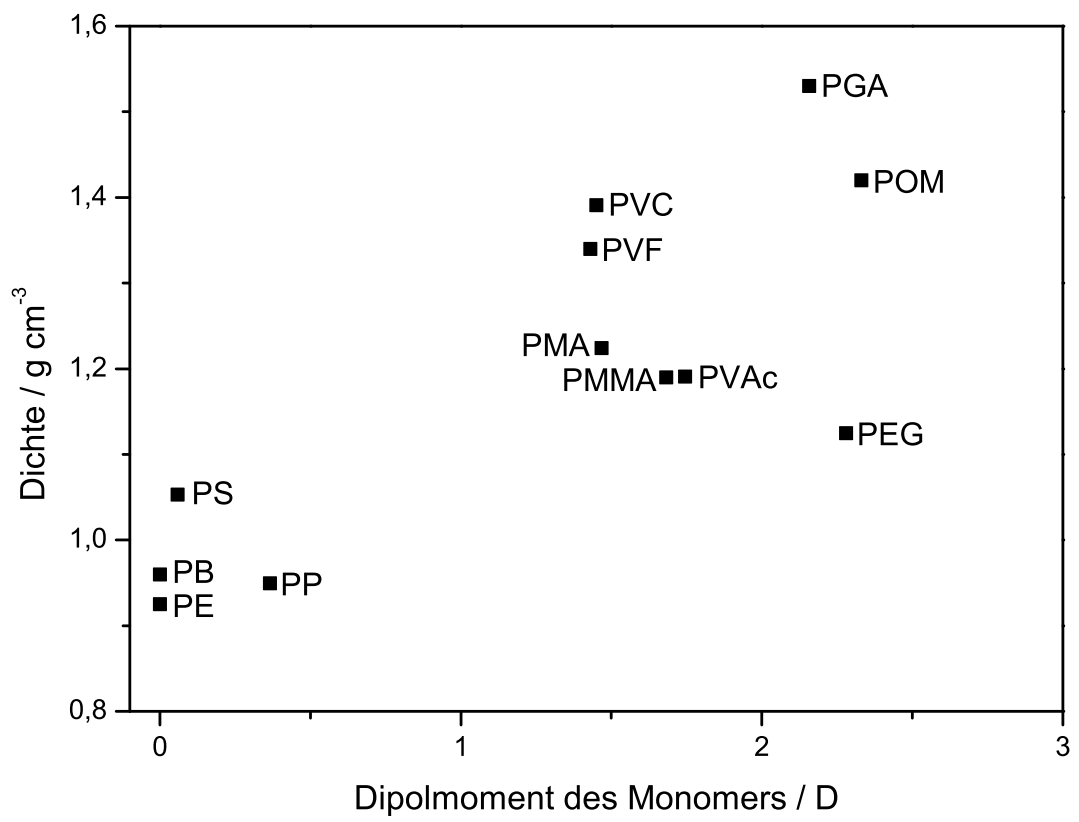

Abbildung 15: Auftragung der Dichte einiger Polymere gegen das Dipolmoment der zugehörigen Monomere. PE: Polyethylen, PB: Polybutadien, PP: Polypropylen, PS: Polystyrol, PMA: Polymethylacrylat, PMMA: Polymethylmethacrylat, PVAc: Polyvinylacetat, PEG: Polyethylenglykol, PVF: Polyvinylfluorid, PVC: Polyvinylchlorid, POM: Polyoxymethylen, PGA: Polyhydroxyessigsäure. Die zugehörige Wertetabelle und Quellen sind in Kapitel 7.2 gezeigt.

Zum einen erhöht sich durch den Schaltprozess das Dipolmoment der Azobenzol-Endgruppen im Polymer. Dadurch können sich attraktive Dipol-Dipol-Wechselwirkungen ausbilden, die zudem langreichweitiger als die im unpolaren Polymer auftretenden Van-der-WaalsWechselwirkungen sind. Die Polymerketten können sich dichter zusammenlagern, die Dichte des Polymers steigt. Ein Vergleich von Dichte und Dipolmoment bekannter Polymere zeigt, dass eine starke Korrelation zwischen beiden Größen besteht, wie in Abbildung $15 \mathrm{zu}$ sehen ist. Zum anderen ändert sich durch die Isomerisierung auch die Geometrie der Azobenzol-Endgruppen. Der endständige Phenylring, der in der trans-Konfiguration vom Polymerrückgrat wegzeigt, richtet sich in 
der cis-Konfiguration zum Polymerückgrat hin. Als Konsequenz würde der sterische Anspruch der Seitengruppe sinken und die Polymerketten können sich besser annähern. Folglich würde das freie Volumen sinken und die Dichte steigen.

Die Schichtdickenänderung bei PAzoMA und bei PAzoPMA ist im Rahmen des Fehlers gleich. Bei PAzoHMA ist sie hingegen mehr als doppelt so groß. Der Effekt der Dipol-Dipol-Wechselwirkung sollte von der Linkerlänge unabhängig sein und für alle drei Polymere den gleichen Beitrag zur Erhöhung der Dichte liefern. Ein großer Unterschied ergibt sich jedoch bei der Geometrieänderung der Azobenzol-Gruppe. Bei PAzoMA und PAzoPMA ist die Azobenzol-Gruppe im Vergleich zu PAzoHMA näher am Polymerrückgrat. Richtet sich die endständige Phenylgruppe am Azobenzol also in Richtung des Polymerrückgrates aus, so könnte sie zuvor freies Volumen einnehmen, welches sich zwischen den Seitenketten des Polymers befand. Da kein Unterschied in der relativen Schichtdickenänderung zwischen PAzoMA und PAzoPMA gefunden wurde, scheint das freie Volumen hier vergleichbar zu sein. Bei PAzoHMA sollte das freie Volumen durch den längeren Linker größer sein. Die Geometrieänderung des Azobenzols wirkt sich hier deutlicher aus, da mehr freies Volumen genutzt werden kann um die Seitenketten effizienter anzuordnen. Die Erhöhung der Dichte ist bei PAzoHMA deshalb am größten.

\subsection{Fazit und weitere Forschungsperspektiven}

Azobenzol-basierte Monomere konnten erfolgreich hergestellt und charakterisiert werden. Durch RAFT-Polymerisation wurden daraus photoschaltbare Polymere synthetisiert. Der Erfolg der Synthesen wurde durch NMR-Spektroskopie, UV/Vis-Spektroskopie, ESI-MS und GPC belegt. Die Kinetik der cis-trans-Isomerisierung in Lösung wurde für alle Mono- und Polymere durch UV/Vis-Spektroskopie untersucht. Dabei wurde der Einfluss von Temperatur, Lösungsmittel, Linkerlänge auf die Reaktionsgeschwindigkeit sowie der Unterschied zwischen Monound Polymer aufgeklärt. Die Reaktionsgeschwindigkeit stiegt für alle 
Proben mit der Temperatur an. Am Beispiel von AzoMA wurde deutlich, dass die Aktivierungsenergie mit der Polarität des Lösungsmittels abnimmt. Zudem wurde gezeigt, dass die Isomerisierungsgeschwindigkeit mit der Polarität zunächst leicht ansteigt, bis sie bei stark polaren Lösungsmitteln deutlich abfällt. Eine vergleichbare Studie über den Einfluss des Lösungsmittels könnte auch für die Polymere interessante Ergebnisse liefern. Die Verteilung der End-zu-End-Abstände wird möglicherweise vom Lösungsmittel beeinflusst und führt so zu einem Einfluss auf die kooperativen Effekte bei der Isomerisierung. Des Weiteren könnte die Polarisierbarkeit des Lösungsmittels einen Einfluss auf die Isomerisierungsrate haben, da sich das Dipolmoment der AzobenzolGruppe in der cis-Konfiguration durch polarisierbare Lösungsmittel weiter ausbreiten könnte, während schlecht polarisierbare Lösungsmittel einen abschirmenden Effekt zeigen könnten, die kooperative Effekte abschwächen würden.

Wird der Einfluss der Linkerlänge betrachtet, so konnte gezeigt werden, dass die Aktivierungsenergie der Isomerisierung hiervon unabhängig ist. Bei konstanter Temperatur steigt die Isomerisierungsgeschwindigkeit für die Monomere mit der Linkerlänge an. Bei den Polymeren steigt sie zunächst an, bei tieferen Temperaturen fällt sie bei einer hohen Linkerlänge wieder leicht ab. Ein Vergleich der Monomere und Polymere zeigt, dass die Aktivierungsenergie bei den Polymeren etwas geringer ist, als die des zugehörigen Monomers. Die Reaktionsgeschwindigkeit ist für PAzoPMA, PAzoHMA und PAzoUMA stets größer als im Monomer. Bei PAzoMA ist sie bei hohen Temperaturen langsamer als im Monomer.

Eine Untersuchung der Schaltkinetik von Polymeren mit unterschiedlichen Kettenlängen könnte den Unterschied im Verhalten von Monomer und Polymer genauer beleuchten. Dabei sind insbesondere sehr kurze und sehr lange Ketten von Interesse um Extremfälle des Systems abzubilden.

In fester Phase wurde eine UV-induzierte Abnahme der Schichtdicke bei photoschaltbaren Polymerdünnfilmen beobachtet. Es konnte ge- 
zeigt werden, dass diese über mehrere Schaltzyklen reversibel ist. Zudem ist der Effekt bei PAzoHMA etwa doppelt so groß wie bei PAzoMA und PAzoPMA. 


\section{Untersuchung von photoschaltbaren Blockcopolymersystemen}

\subsection{Hintergründe}

Polymere, welche aus mehreren verschiedenen Monomerarten aufgebaut sind, werden als Copolymere bezeichnet. Neben statistischen Copolymeren, bei denen die Anordnung der Monomereinheiten zufällig ist, oder alternierenden Copolymeren, bei denen die Monomere stets einander abwechseln, gibt es auch Blockcopolymere, die aus mindestens zwei Blöcken von Homopolymeren bestehen. ${ }^{75}$ Dabei sind verschiedene Monomerzusammensetzungen und daraus resultierend auch eine Vielzahl an Topologien möglich, die in Abb. 16 gezeigt sind. ${ }^{76,77}$

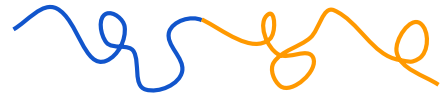

Diblockcopolymer [AB]

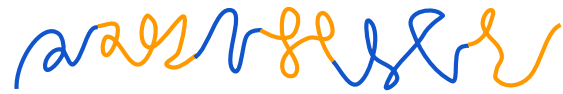

Multiblockcopolymer [ABABAB]

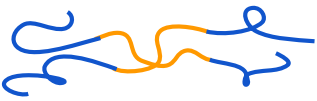

Sternblockcopolymer

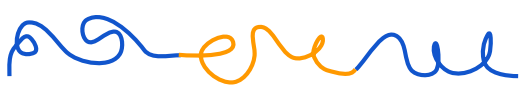

Triblockcopolymer [ABA]

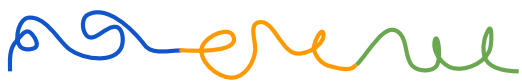

Triblockcopolymer [ABC]

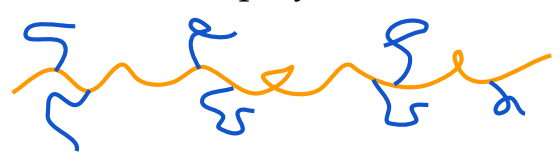

Pfropfblockcopolymer

Abbildung 16: Schematische Darstellung von verschiedenen Blockcopolymerarten. ${ }^{36}$

Da die Blöcke eines Blockcopolymers aus verschiedenen Monomeren aufgebaut sind, weisen sie in der Regel unterschiedliche Eigenschaften auf. Dies kann zu einer Entmischung des Systems führen, wenn die Blöcke eine schlechte Mischbarkeit miteinander zeigen. Durch die physikalische Verknüpfung zwischen den beiden Blöcken ist eine Entmi- 
schung des Systems auf makroskopischer Skala unmöglich. Es kommt daher zur Ausbildung sogenannter Mikrophasen, die eine Größe in der Größenordnung von 10-100 nm aufweisen. ${ }^{78,79}$

Eine Betrachtung der Thermodynamik eines Blockcopolymers liefert dabei wichtige Informationen für das Verhalten eines solchen Systems. Die Gibbs-Energie eines Blockcopolymers $\Delta G_{\mathrm{BCP}}$ kann durch

$$
\Delta G_{\mathrm{BCP}}=\Delta H_{\mathrm{m}}-T \Delta S_{\mathrm{k}}
$$

beschrieben werden. Dabei ist $\Delta H_{\mathrm{m}}$ die Mischungsenthalpie, $T$ die Temperatur und $\Delta S_{\mathrm{k}}$ die Konformationsentropie. Ist $\Delta G_{\mathrm{BCP}}$ negativ, so ist eine Mischung beider Blöcke zu einer homogenen Phase bevorzugt. Bei einem positiven $\Delta G_{\mathrm{BCP}}$ strebt das System eine Mikrophasenseparation an. Die Mischungsenthalpie ist durch

$$
\Delta H_{\mathrm{m}}=\mathrm{R} T \chi f_{\mathrm{A}} f_{\mathrm{B}}
$$

gegeben. ${ }^{80,81}$ Dabei ist $\mathrm{R}$ die universelle Gaskonstante, $\chi$ der Interaktionsparameter und $f_{\mathrm{A}}$ sowie $f_{\mathrm{B}}$ die Volumenanteile der Komponenten A und B. Der Interaktionsparameter $\chi$ wird beschrieben durch:

$$
\chi=\left(\frac{z}{k_{\mathrm{B}} T}\right)\left(\varepsilon_{\mathrm{AB}}-\frac{1}{2}\left(\varepsilon_{\mathrm{AA}}+\varepsilon_{\mathrm{BB}}\right)\right) .
$$

Dabei ist $z$ die Anzahl benachbarter Monomereinheiten pro Monomereinheit und $k_{\mathrm{B}}$ die Boltzmannkonstante. Die Interaktionsenergien zwischen zwei Monomerarten (A-A, A-B oder B-B) werden mit $\varepsilon_{\mathrm{AA}}, \varepsilon_{\mathrm{AB}}$ und $\varepsilon_{\mathrm{BB}}$ bezeichnet. Der Interaktionsparameter beschreibt die Wechselwirkung zwischen beiden Komponenten des Systems.

Die Konformationsentropie des Systems wird im Wesentlichen durch zwei Faktoren bestimmt. An der Grenzfläche der beiden Phasen sind die Polymere gestreckt, wodurch sie von der entropisch günstigsten Konformation eines Gauß'schen Knäuls abweichen. Zudem ordnet die Ausbildung einer Phasengrenze die Verknüpfungspunkte zwischen den Blöcken auf einer Grenzfläche an, was die Anordnungsfreiheit des 
Systems einschränkt. Dieses liefert zum erstgenannten Effekt jedoch lediglich einen kleinen Beitrag zur Konformationsentropie. ${ }^{82}$

Um eine Mikrophasenseparation zu erzielen, ist ein positives $\Delta G_{\mathrm{m}}$ erforderlich. Dafür sind aus thermodynamischen Gesichtspunkten folgende Kriterien entscheidend: Der Enthalpietherm muss groß und der Entropieterm klein sein. Ein großer Enthalpieterm wird für hohe Interaktionsparameter erhalten, der Entropieterm wird bei tiefen Temperaturen und hohen Polymerisationsgraden minimiert. Das Produkt aus Interaktionsparameter und Polymerisationsgrad $\chi N$ wird oft als Größe herangezogen, um den Zustand eines Blockcopolymers bei konstanter Temperatur zu charakterisieren. Ab einem Wert von $\chi N \approx 10,5$ beginnt die Mikrophasenseparation. ${ }^{79,83}$ Die Blöcke sind dabei jedoch noch nicht sehr klar voneinander abgegrenzt. Dies wird als Weak Segregation Limit (WSL) bezeichnet.

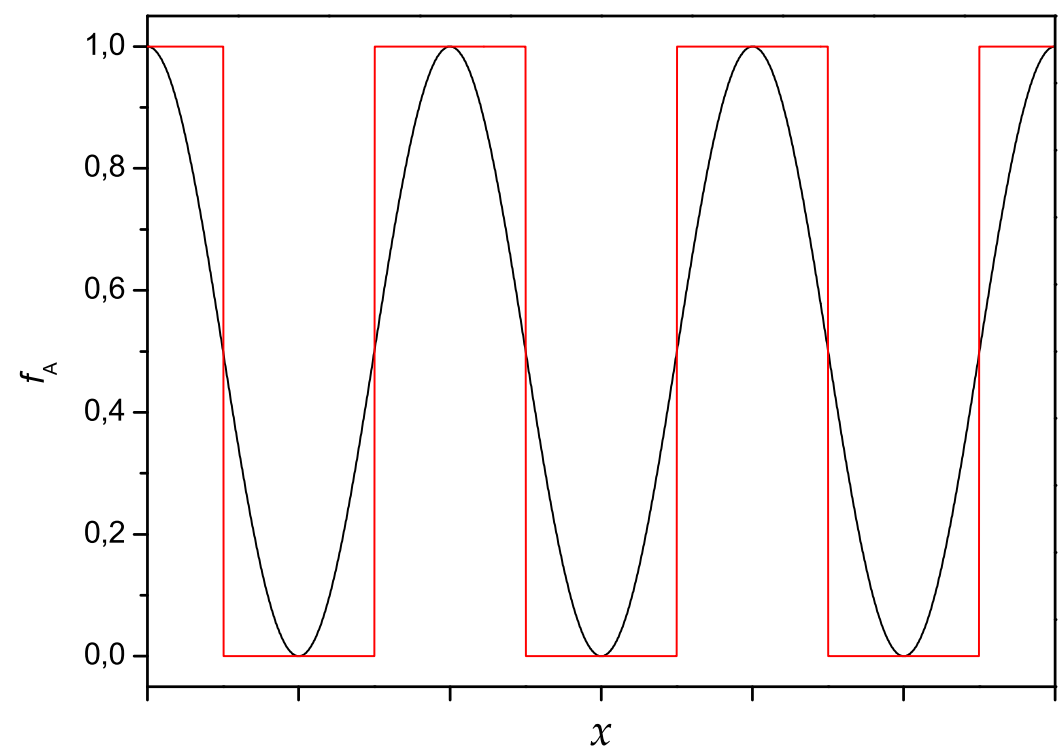

Abbildung 17: Schematische Darstellung eines eindimensionalen Konzentrationsprofils gegen die Querschnittskooordinate $x$ eines in lamellarer Morphologie mikrophasenseparierten Blockcopolymers am Weak Segregation Limit (schwarz) und am Strong Segregation Limit (rot). ${ }^{36}$ 

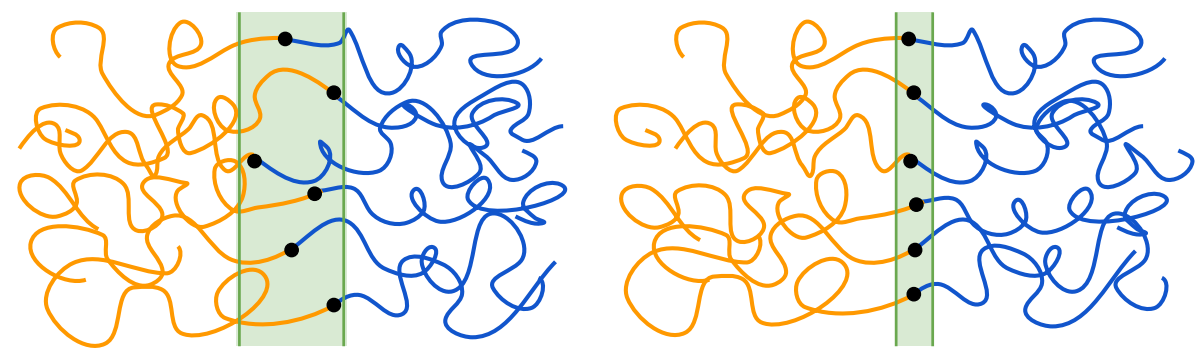

Abbildung 18: Schematische Darstellung der Grenzfläche (grün) zwischen zwei Phasen eines mikrophasenseparierten Blockcopolymers am WSL (links) und SSL (rechts). ${ }^{36}$

Mit zunehmendem $\chi N$ wird die Entmischung der Blöcke aufgrund der steigenden Unverträglichkeit stärker, die Phasen grenzen sich schärfer voneinander ab. Dieser Fall wird als Strong Segregation Limit (SSL) bezeichnet. Das Konzentrationsprofil eines Blockcopolymers am WSL und SSL ist schematisch in Abbildung 17 gezeigt. Der Unterschied zwischen WSL und SSL wird bei einer Betrachtung der Grenzfläche deutlicher, wie in Abbildung 18 gezeigt ist. Die Grenzfläche ist der Bereich, in dem sich alle Verknüpfungspunkte der beiden Blöcke des Blockcopolymers befinden. Eine dünnere Grenzfläche bietet die Möglichkeit einer enthalpisch günstigeren Anordnung der Polymerketten, da der Kontakt unterschiedlicher Blöcke minimiert wird. Dem stehen weniger Anordnungsmöglichkeiten der Verknüpfungspunkte entgegen, weshalb eine dünne Grenzfläche entropisch ungünstig ist. Zudem ist eine Streckung der Ketten in der Nähe des Verknüpfungspunktes notwendig. Eine dünne Grenzfläche ist somit nur für große $\chi N$ möglich, da der enthalpische Gewinn groß ist, während der entropische Verlust klein bleibt. Deshalb ist im Falle des WSL ist die Grenzfläche relativ breit, während sie im Fall des SSL schmal ist. ${ }^{79}$

Um Informationen über das Phasenverhalten von Blockcopolymeren zu erlangen, wurden seit den 70er-Jahren Molekularfeldtheorien und Berechnungen entwickelt, mit denen die Morphologien von mikrophasenseparierten Blockcopolymeren vorhergesagt werden konnten. ${ }^{84-92}$ Mat- 
sen und Bates veröffentlichten 1996 ein berechnetes Phasendiagramm, welches in Abbildung 19 dargestellt ist.

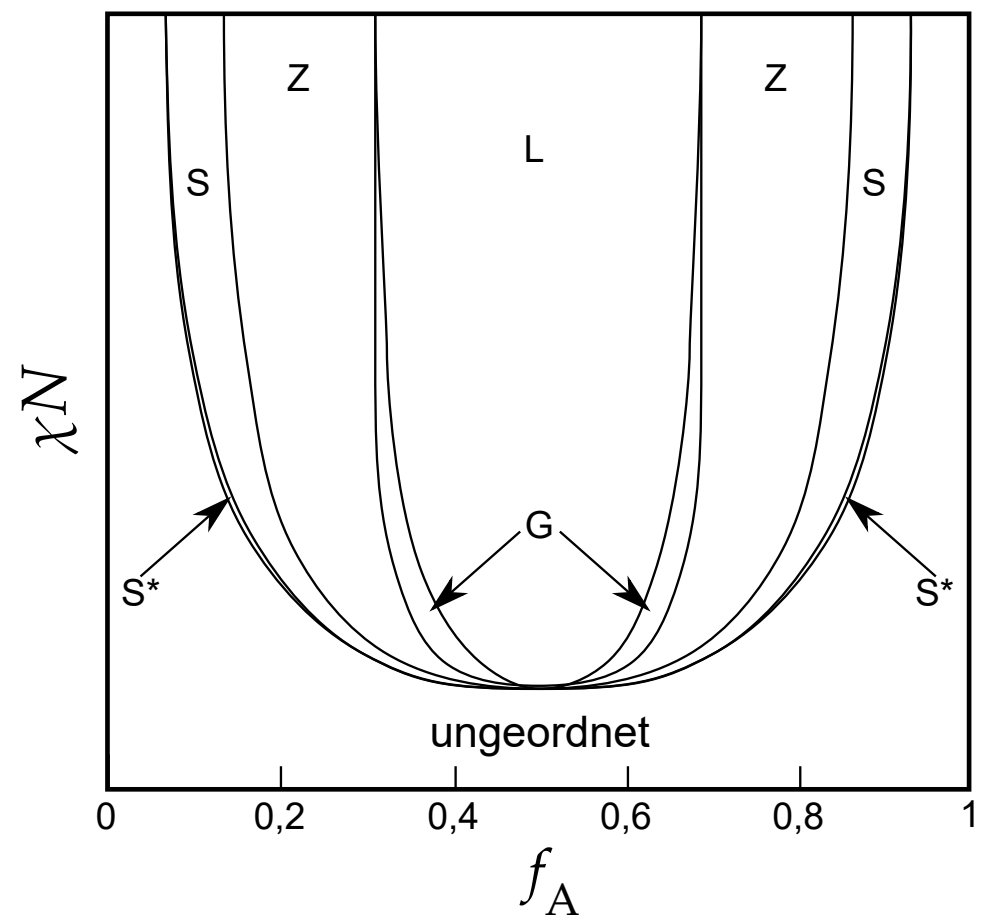

Abbildung 19: Theoretisches Phasendiagramm eines Blockcopolymers nach Matsen und Bates ${ }^{83}$ in Abhängigkeit von Volumenanteil der Komponenten und $\chi N$. Die Buchstaben stehen für die Bezeichnung der jeweiligen Phase: Dicht gepackte Kugeln $\left(\mathrm{S}^{*}\right)$, kubisch innenzentrierte Kugeln (S), hexagonal angeordnete Zylinder (Z), Gyroid (G) sowie Lamellen (L). ${ }^{36}$
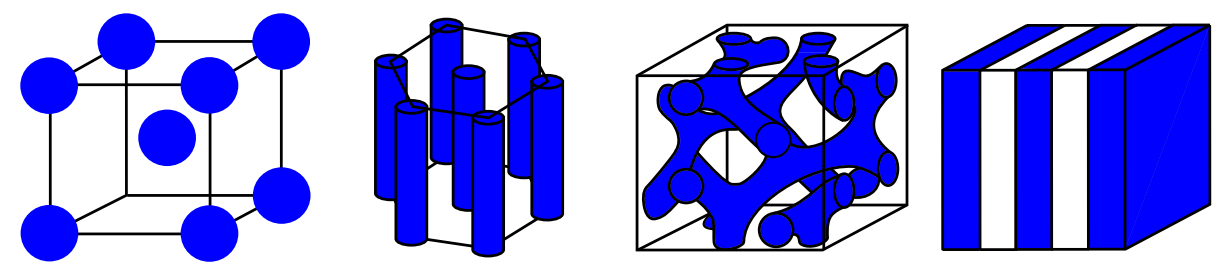

Abbildung 20: Schematische Darstellung der unterschiedlichen Morphologien der Mikrophasenseparation. Von links nach rechts: Kugeln, Zylinder, Gyroid, Lamellen. ${ }^{36}$ 
Ist $\chi N$ sehr klein, oder der Volumenanteil einer Komponente sehr groß, so liegt ein ungeordneter Zustand vor. Ein ungeordneter Zustand kann auch erreicht werden, indem ein mikrophasensepariertes Blockcopolymersystem erhitzt wird. Mit steigender Temperatur sinkt $\chi N$ gemäß Gleichung 12, sodass ab einer bestimmten Temperatur die Phasengrenze zum ungeordneten Zustand überschritten wird. Diese Temperatur wird als Ordnungs-Unordnungs-Übergangstemperatur $T_{\mathrm{ODT}}$ bezeichnet.

Welche Morphologie sich im Falle einer Mikrophasenseparation ausbildet hängt vom Volumenanteil der Komponenten, deren Polymerisationsgrad und dem Interaktionsparameter ab. Diese Parameter bestimmen die Form der Grenzfläche maßgeblich welche sich wiederum auf die Geometrie der gebildeten Morphologie auswirkt. Dies ist in Abbildung 21 verdeutlicht. Mit steigendem Volumenanteil $f_{\mathrm{A}}$ der Komponente A verändert sich die Krümmung der Grenzfläche, die Morphologie geht von Kugeln zu Zylindern in Lamellen über.
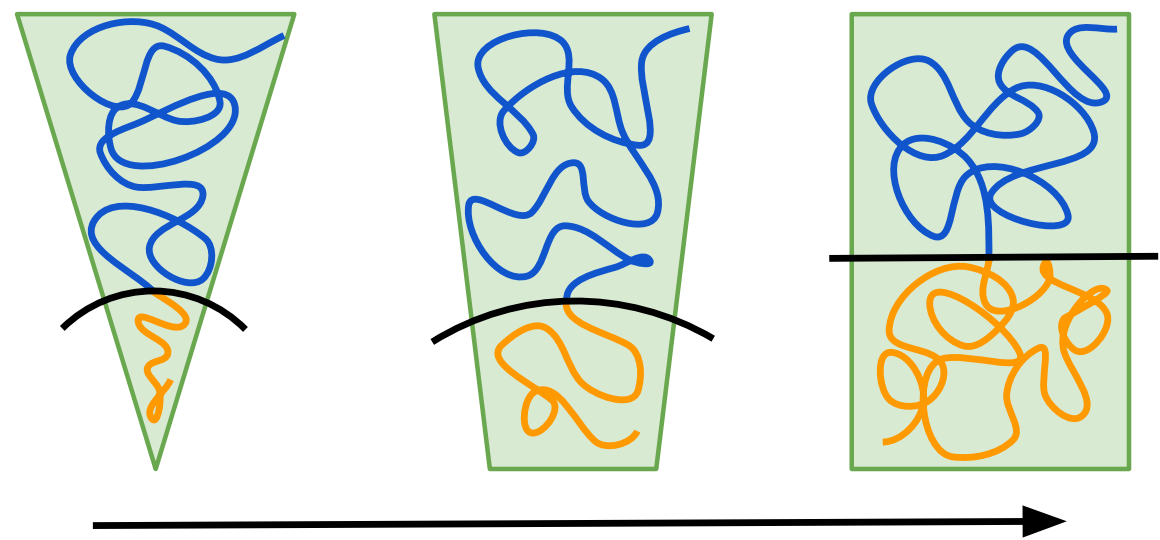

$f_{\mathrm{A}}$

Abbildung 21: Schematische Darstellung des Einflusses des Volumenanteils von Komponente A (orange) $f_{\mathrm{A}}$ auf die Form der Phasengrenzfläche (schwarz) und der Geometrie der gebildeten Morphologie der Mikrophasenseparation. ${ }^{36}$ 


\subsection{Synthese photoschaltbarer Blockcopolymere}

In früheren Arbeiten wurde das photoschaltbare Blockcopolymersystem PAzoPMA- $b$-PBA erstmalig untersucht. $^{36}$ Bei einem Blockcopolymer (BCP I) mit einer mittleren Molmasse von $M_{\mathrm{n}}=4,6 \cdot 10^{4} \mathrm{~g} \cdot \mathrm{mol}^{-1}$, einer Dispersität von $Ð=1,49$ und einem Stoffmengenanteil an AzoPMA von 18 \% wurde die Mikrophasenseparation charakterisiert. Sie wurde einer lamellaren Morphologie zugeschrieben, welches jedoch nicht abschließend belegt werden konnte. Zudem konnte gezeigt werden, dass sich bei senkrecht stehenden Lamellen die Höhendifferenz reversibel durch das Isomerisieren der Azobenzol-Gruppe zwischen zwei Zuständen schalten lässt.

Ausgehend von diesen Ergebnissen soll das System PAzoPMA- $b$-PBA weiter untersucht werden. Der Fokus soll dabei insbesondere auf dem Maßschneidern der Phasengrößen bei einer lamellaren Morphologie liegen. Im Folgenden werden zwei Varianten, die dies ermöglichen, vorgestellt. Eine Möglichkeit besteht darin, die Blocklänge eines Blockes so zu variieren, dass die Morphologie der Mikrophasenseparation erhalten bleibt, die Größe einer der beiden Phasen sich jedoch ändert. Der zweite Ansatz besteht darin, beide Phasen eines Blockcopolymers mit Homopolymer gleichmäßig aufzuschwellen, sodass der Volumenanteil beider Komponenten konstant bleibt, um den Erhalt der Morphologie zu gewährleisten.

Die Herstellung der Blockcopolymere erfolgte durch RAFTPolymerisation. Im ersten Schritt wurde dazu AzoPMA als Monomer mit CDSPA als RAFT-Agens und ACCN als Initiator in DMF umgesetzt und PAzoPMA erhalten (Kapitel 6.8). Die Molmassenverteilung wurde durch GPC bestimmt und ist in Abbildung 22 gezeigt. Es wurde eine schmale, monomodale Molmassenverteilung erhalten. Das Polymer weist eine mittlere Molmasse von $M_{\mathrm{n}}=1,7 \cdot 10^{4} \mathrm{~g} \cdot \mathrm{mol}^{-1}$ und eine Dispersität von $Đ=1,38$ auf. Durch die endständige RAFT-Gruppe handelt es sich bei dem Polymer um ein Makro-RAFT-Agens, welches in einem zweiten Syntheseschritt zu einem Blockcopolymer umgesetzt werden kann. Durch Umsetzung mit unterschiedlichen Äquivalenten 
an n-Butylacrylat mit AIBN als Initiator in DME wurden drei verschiedene Blockcopolymere erhalten (BCP II, BCP III und BCP IV). Auch hier wurden die Molmassenverteilungen durch GPC bestimmt und in Abbildung 22 dargestellt.

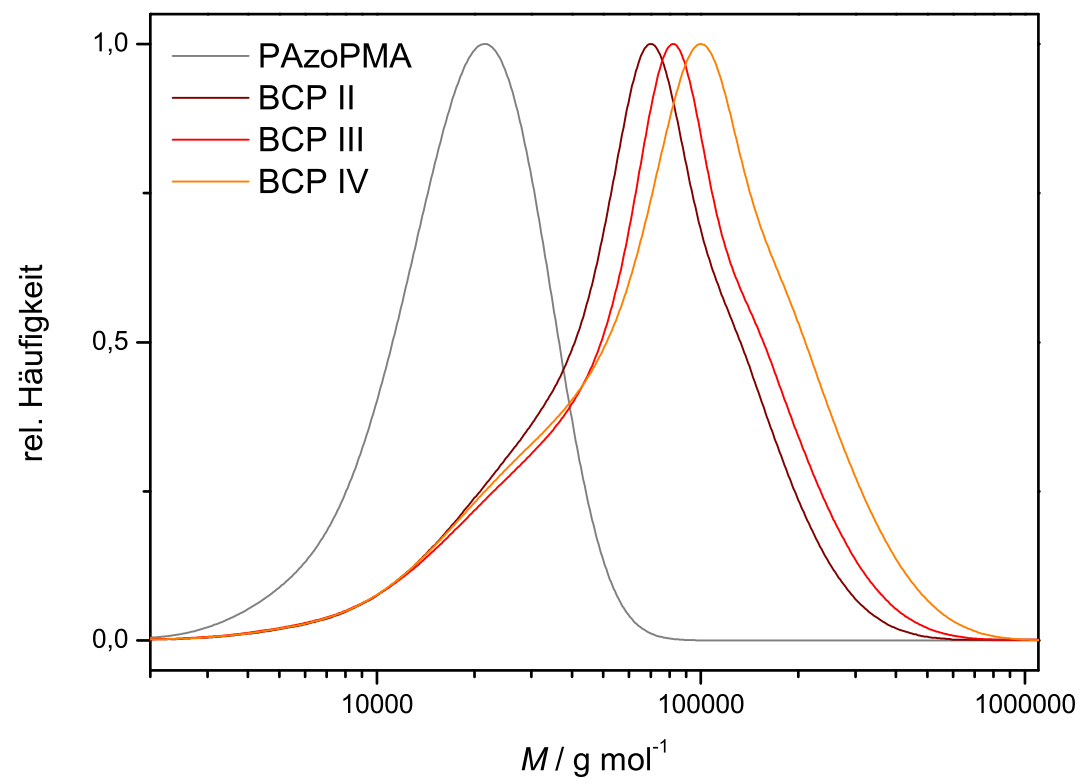

Abbildung 22: Aus dem GP-Chromatogramm berechnete normierte Molmassenverteilungen vom Homopolymer PAzoPMA sowie den Blockcopolymeren BCP II-IV.

Die Zusammensetzung der Blockcopolymere wurde durch den Vergleich von Integralen aus den ${ }^{1} \mathrm{H}-\mathrm{NMR}-S p e k t r e n$ bestimmt. Für jedes Blockcopolymer wurde ein Spektrum in deuteriertem Dichlormethan aufgenommen. Diese sind in Abbildung 23 gezeigt. Das Signal bei 7,73 ppm (blaue Markierung) ist dem para-substituierten Phenylring der Azobenzolgruppe im AzoPMA zuzuordnen. Das Signal bei 0,86 ppm (rote Markierung) stammt von der endständigen Methylgruppe der n-Butyl-Gruppe aus dem Butylacrylat. Aus den Integralen des Signals aus dem Azobenzol $I_{\mathrm{Azo}}$ und dem der Methylgruppe $I_{\mathrm{Me}}$ lässt sich der Stoffmengenanteil an AzoPMA $\chi_{\mathrm{Azo}}$ und Butylacrylat $\chi_{\mathrm{BA}}$ berechnen. 


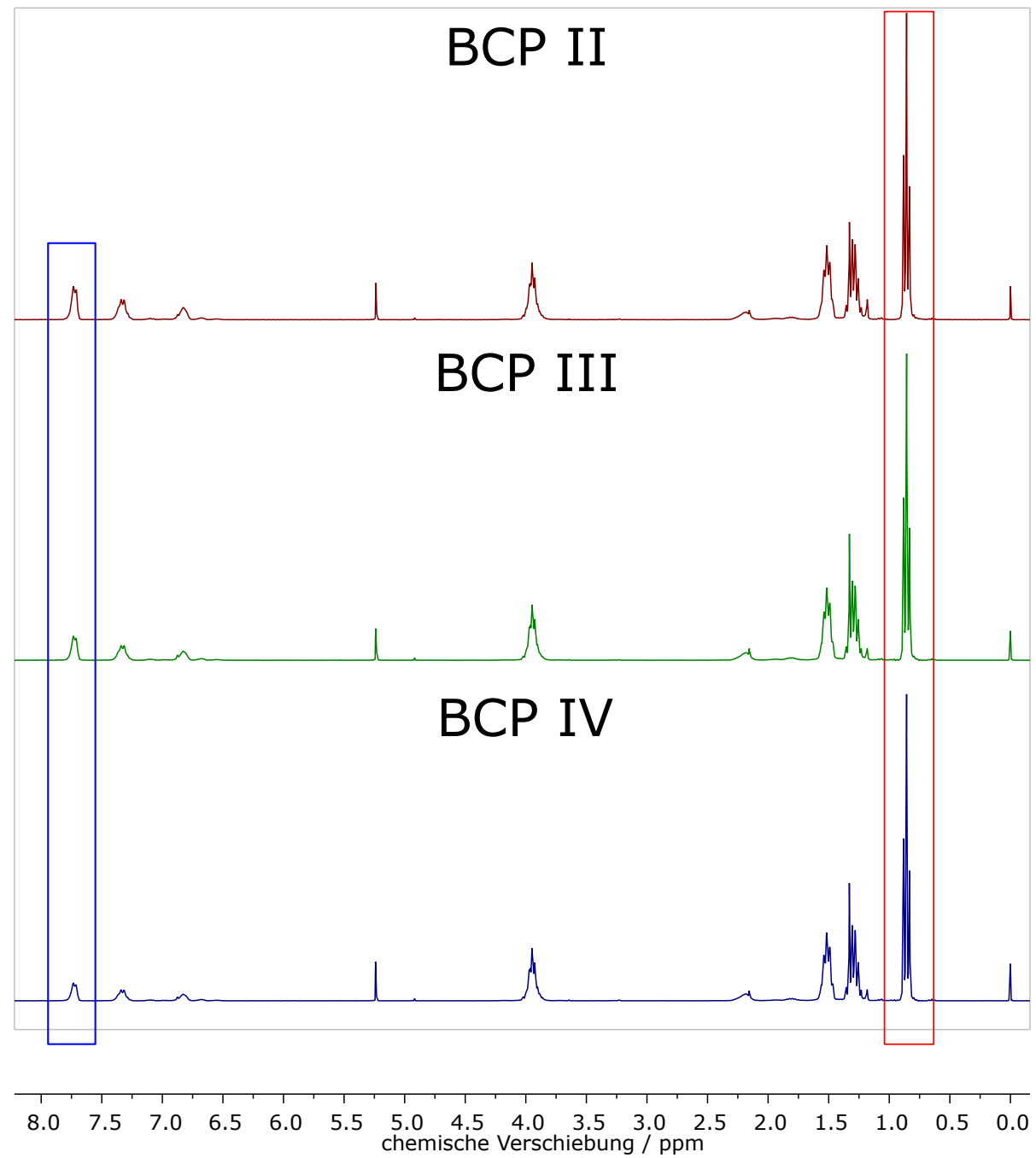

Abbildung 23: ${ }^{1} \mathrm{H}-\mathrm{NMR}-\mathrm{Spektren}$ von BCP II-IV in $\mathrm{CD}_{2} \mathrm{Cl}_{2}$.

Dazu werden die Integrale durch die Anzahl Protonen geteilt, von denen sie hervorgerufen werden, um sie auf die Stoffmenge zu normieren. Der Stoffmengenanteil an AzoPMA kann dann durch

$$
\chi_{\mathrm{Azo}}=\frac{\frac{I_{\mathrm{Azo}}}{4}}{\frac{I_{\mathrm{Azo}}}{4}+\frac{I_{\mathrm{Me}}}{3}}
$$


berechnet werden. Wird $I_{\text {Azo }}$ als 4 definiert, vereinfacht sich der Ausdruck zu

$$
\chi_{\mathrm{Azo}}=\frac{1}{1+\frac{I_{\mathrm{Me}}}{3}} .
$$

Der Stoffmengenanteil an Butylacrylat berechnet sich durch:

$$
\chi_{\mathrm{BA}}=1-\chi_{\mathrm{Azo}} .
$$

Die bestimmten Integrale sowie die berechneten Stoffmengenanteile nach Gleichung 14 und die Ergebnisse der GPC sind in Tabelle 7 zusammengefasst.

Tabelle 7: Mittlere Molmassen $M_{\mathrm{n}}$, Dispersität $\oslash$ sowie Stoffmengenanteile an AzoPMA $\chi_{\mathrm{Azo}}$ und BA $\chi_{\mathrm{BA}}$ für BCP II-IV.

\begin{tabular}{lccccc}
\hline Polymer & $M_{\mathrm{n}} /\left(10^{4} \mathrm{~g} \cdot \mathrm{mol}^{-1}\right)$ & $Ð$ & $I_{\text {Me }}$ & $\chi_{\text {Azo }}$ & $\chi_{\text {BA }}$ \\
\hline BCP II & 4,33 & 1,8 & 14,28 & 0,17 & 0,83 \\
BCP III & 4,71 & 1,9 & 19,09 & 0,14 & 0,86 \\
BCP IV & 5,37 & 2,1 & 24,14 & 0,11 & 0,89 \\
\hline
\end{tabular}

\subsection{Untersuchung der Mikrophasenseparation photoschaltbarer Blockcopolymere}

Zur Untersuchung der Mikrophasenseparation wurden die Blockcopolymere in Propylenglycolmonomethlyetheracetat (PGMEA) gelöst und auf Siliciumwafer gespincoated. Das Oberflächenprofil der ungetemperten Proben wurde anschließend mit dem Rasterkraftmikroskop (AFM) gemessen. Die Höhenprofile sind in Abbildung 24 gezeigt. Es wird deutlich, dass für alle Proben bereits der eine Mikrophasenseparation erkennbar ist, welche jedoch noch keiner Morphologie zugeordnet werden kann. Es handelt sich dabei um eine Struktur, die durch das schnelle Verdampfen des Lösungsmittels beim Spincoaten kinetisch eingefangen wurde und das System somit nicht die energetisch günstigste Anordnung der Mikrophasenseparation annehmen konnte. 

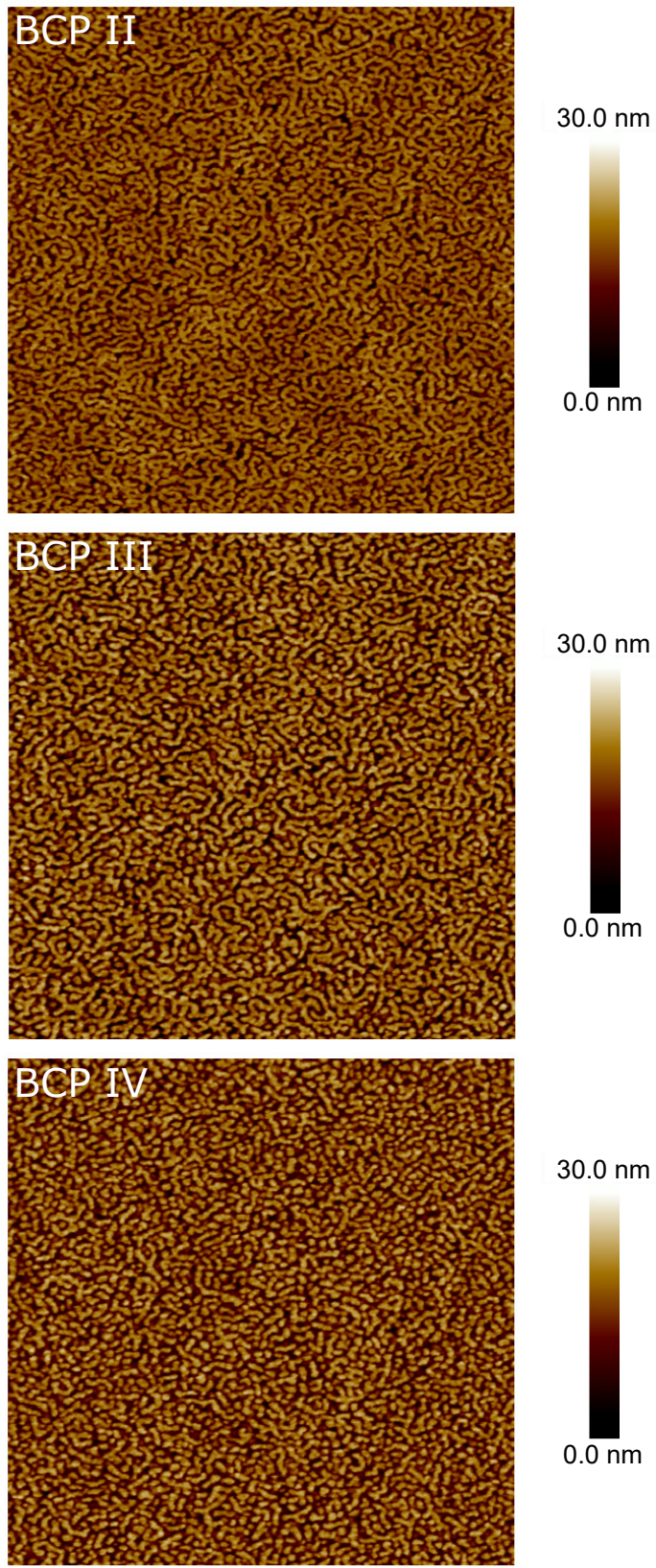

$30.0 \mathrm{~nm}$

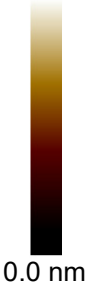

Abbildung 24: AFM-Aufnahmen der Höhenprofile von BCP II-IV. Die Proben wurden auf Si-Wafer gespincoatet. Ausschnitt: $2 \times 2 \mu \mathrm{m}$, Auflösung: $512 \times 512$ Pixel. 


\subsubsection{Zeitliche Untersuchung des Temperprozesses}

Um weitere Erkenntnisse über die Struktur zu gewinnen, wurde der Prozess des thermischen Temperns genauer untersucht. Dazu wurden Proben des Blockcopolymers BCP III gespincoatet und im Anschluss für unterschiedliche Zeiten bei $180{ }^{\circ} \mathrm{C}$ getempert. Die Höhenprofile wurden durch AFM bestimmt und sind in Ausschnitten in Abbildung 25 gezeigt. Die vollständigen Bilder sind in Abbildung 63 in Kapitel 7.3 aufgeführt. Bereits nach fünfminütigem Tempern ist eine deutliche Veränderung der Oberflächenstruktur zu erkennen. Die mikrophasenseparierten Bereiche schließen sich zu übergeordneten Strukturen zusammen, sodass lamellare Anordnungen über die gesamte Probenoberfläche hinweg zu beobachten sind. Zudem bilden sich kleinere Bereiche, in denen eine homogene, unstrukturierte Oberfläche vorliegt aus. Diese werden mit zunehmender Dauer des Tempervorganges größer, bis sie sich zu großen Ebenen zusammenschließen und senkrecht angeordnete Lamellen kaum noch vorliegen.

Wird die Oberflächenstruktur auf der Mikrometerskala betrachtet, so sind ebenfalls deutliche Änderungen zu erkennen. Im ungetemperten Zustand ist die Oberfläche über mehrere Mikrometer gesehen, unter Vernachlässigung der Strukturen im Nanometer-Maßstab, konstant und weist keine signifikanten Höhenänderungen auf. Nach fünfminütigem Tempern liegen hingegen Höhenunterschiede in der Größenordnung von etwa $10 \mathrm{~nm}$ vor. Dieser Effekt wird bei längerem Tempern deutlicher. Bei 10-30-minütigem Tempern werden die Höhenunterschiede größer, nach 60-minütigem Tempern hat sich eine homogene Ebene mit mehreren Plateaus gleicher Höhe ausgebildet. Diese Struktur bleibt auch bei längerem Tempern erhalten, sodass es sich um eine Gleichgewichtsstruktur handeln sollte. Die Höhendifferenz zwischen Ebene und Plateau ist zudem in der selben Größenordnung wie der Abstand der vertikal angeordneten Lamellen. Somit ist davon auszugehen, dass es sich bei der Struktur um horizontal angeordnete Lamellen handelt und dass die Plateaus durch eine unvollständige Ausbildung der obersten Lamelle zustande kommen. 


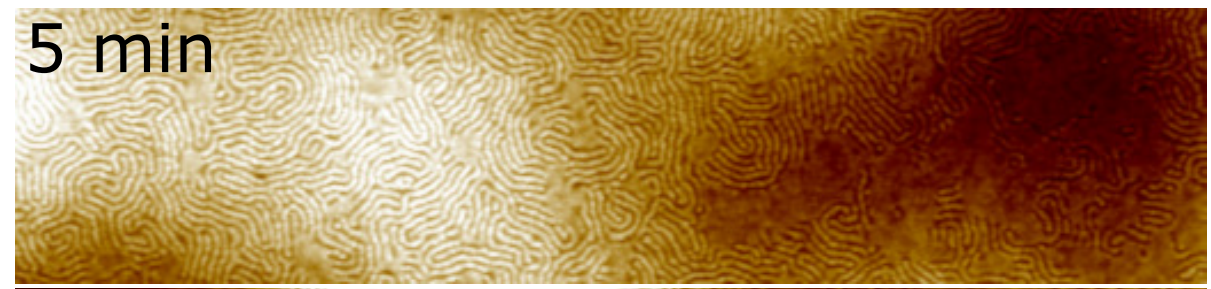

\section{$10 \mathrm{~min}$}

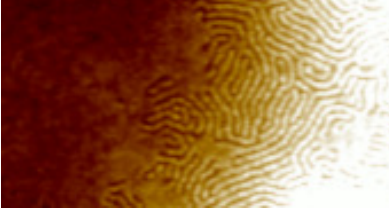

\section{$15 \mathrm{~min}$}

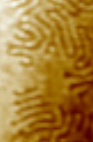

\section{$30 \mathrm{~min}$}

\section{$60 \min$}

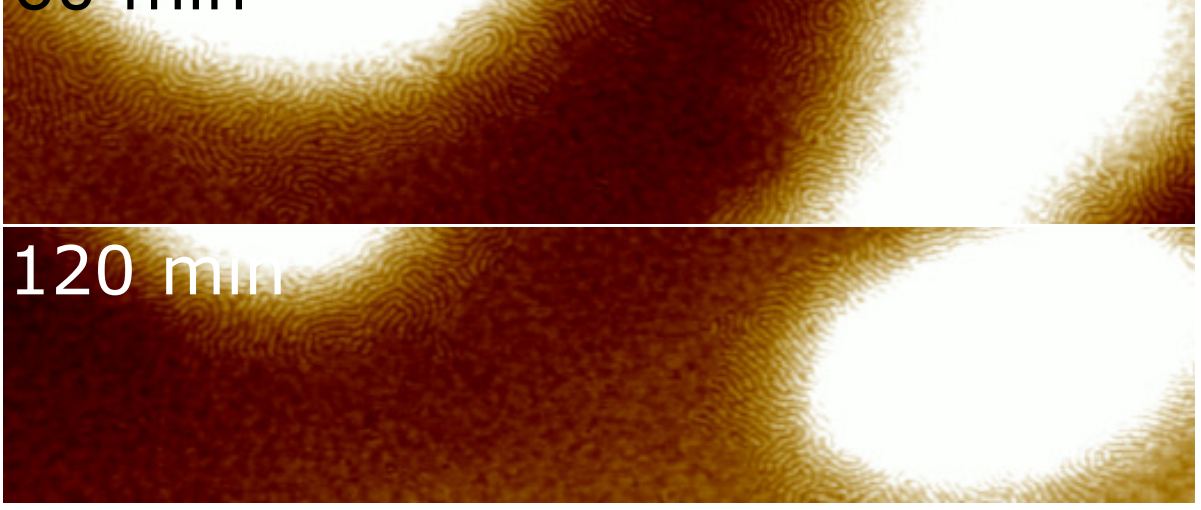

Abbildung 25: AFM-Aufnahmen der Höhenprofile von BCP III nach unterschiedlich langem Tempern bei $180{ }^{\circ} \mathrm{C}$. Ausschnitt: $5,3 \times$ 1,2 $\mathrm{m}$, Auflösung: $512 \times 118$ Pixel. 
Dass die Plateaus erst durch den Temperprozess entstehen, bestärkt die Annahme weiter, dass es sich bei der Morphologie um Lamellen handelt.

\subsubsection{Untersuchung der Mikrophasenseparation von BCP II-IV}

Um die Mikrophasenseparation zu untersuchen, wurden die durch Spincoating hergestellten Proben von BCP II-IV bei $180^{\circ} \mathrm{C}$ für $24 \mathrm{~h}$ getempert. Im Anschluss wurde das Höhenprofil sowie die mechanischen Eigenschaften der Proben mit dem AFM bestimmt. Die erhaltenen Bilder sind in den Abbildungen 26-28 gezeigt. Alle Blockcopolymerproben weisen eine lamellare Morphologie der Mikrophasenseparation auf, bei der Bereiche mit horizontal als auch mit vertikal angeordneten Lamellen vorliegen.

Eine Betrachtung der mechanischen Eigenschaften anhand des DMTModuls zeigt, dass sich im Bereich der vertikalen Lamellen die mechanischen Eigenschaften deutlich unterscheiden. Die homogenen Flächen bestehen aus dem härteren der Materialien. Ein Vergleich mit den Glasübergangstemperaturen $T_{\mathrm{g}, 1}$ und $T_{\mathrm{g}, 2}$ der Komponenten des Blockcopolymers ermöglicht eine Zuordnung der Blöcke. Sie wurden durch dynamische Differenzkalorimetrie bestimmt und sind in Tabelle 8 aufgeführt.

Tabelle 8: Glasübergangstemperaturen $T_{\mathrm{g}, 1}$ und $T_{\mathrm{g}, 2}$ von BCP II-IV.

\begin{tabular}{lcc}
\hline Polymer & $T_{\mathrm{g}, 1} /{ }^{\circ} \mathrm{C}$ & $T_{\mathrm{g}, 2} /{ }^{\circ} \mathrm{C}$ \\
\hline BCP II & 12,4 & 69,0 \\
BCP III & 11,6 & 68,9 \\
BCP IV & 10,8 & 69,0 \\
\hline
\end{tabular}

Der Glasübergang von reinem PAzoPMA $\left(M_{\mathrm{n}}=1,2 \cdot 10^{4} \mathrm{~g} \cdot \mathrm{mol}^{-1}\right)$ liegt bei $T_{\mathrm{g}}=64,2{ }^{\circ} \mathrm{C}$. Damit kann $T_{\mathrm{g}, 1}$ dem BA-Block, $T_{\mathrm{g}, 2}$ dem AzoPMABlock zugeordnet werden. Die homogenen Flächen bestehen daher aus dem PAzoPMA-Block. 


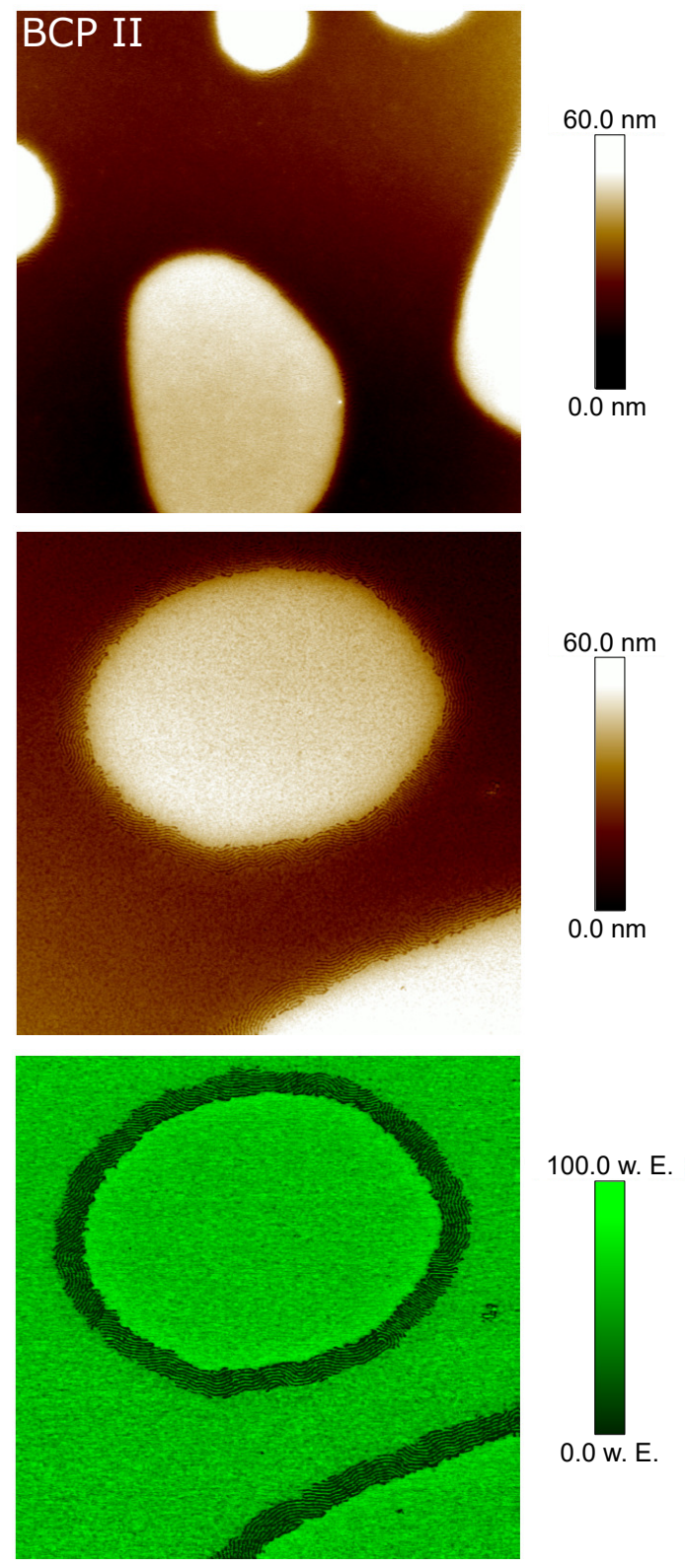

Abbildung 26: AFM-Aufnahmen des Höhenprofils, Ausschnitt: $16 \times 16 \mu \mathrm{m}$ (Oben), Ausschnitt: 5,3 × 5,3 $\mu \mathrm{m}$ (Mitte) und DMT-Modul, Ausschnitt: $5,3 \times 5,3 \mu \mathrm{m}$ (Unten) von BCP II nach Tempern bei $180{ }^{\circ} \mathrm{C}$ für $24 \mathrm{~h}$. Auflösung: $512 \times 512$ Pixel. 

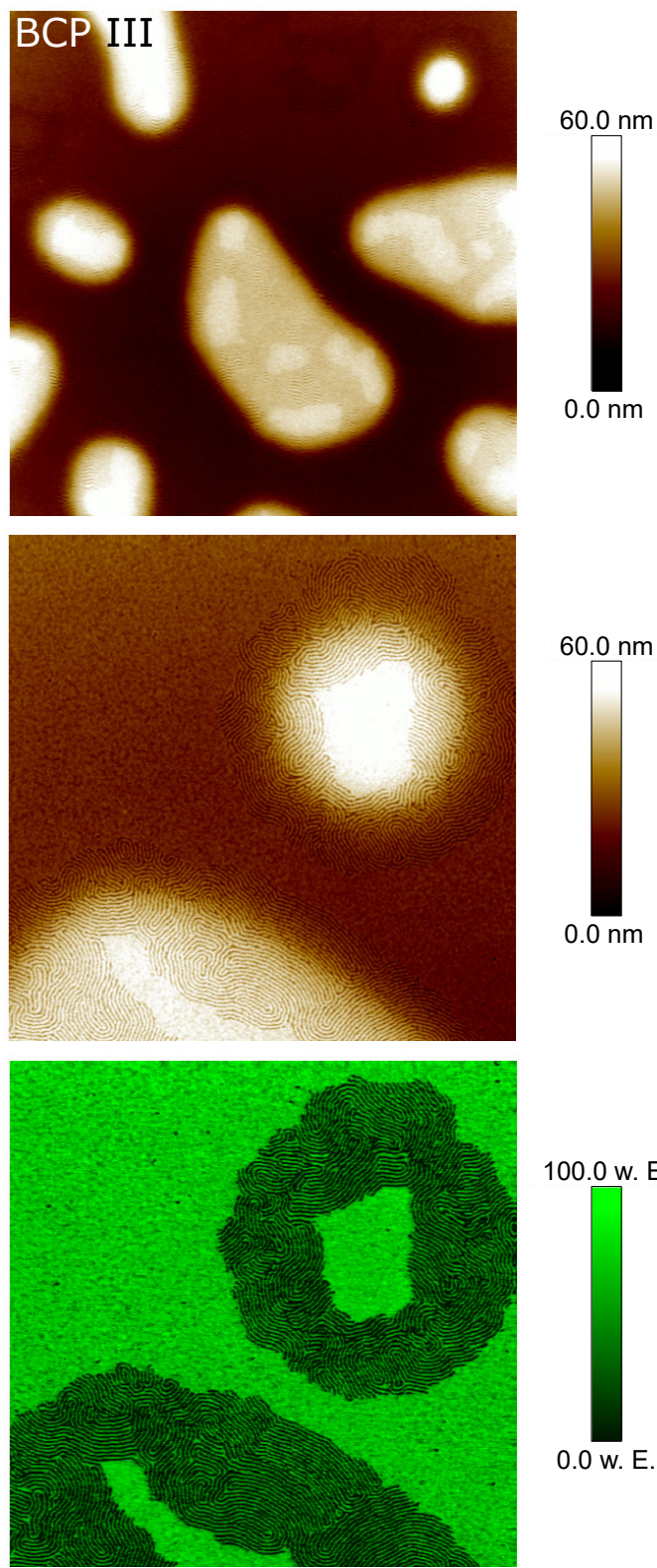

100.0 w. E.

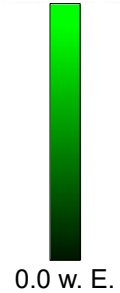

Abbildung 27: AFM-Aufnahmen des Höhenprofils, Ausschnitt: $16 \times 16 \mu \mathrm{m}$ (Oben), Ausschnitt: 5,3 × 5,3 $\mathrm{m}$ (Mitte) und DMT-Modul, Ausschnitt: $5,3 \times 5,3 \mu \mathrm{m}$ (Unten) von BCP III nach Tempern bei $180{ }^{\circ} \mathrm{C}$ für $24 \mathrm{~h}$. Auflösung: $512 \times 512$ Pixel. 

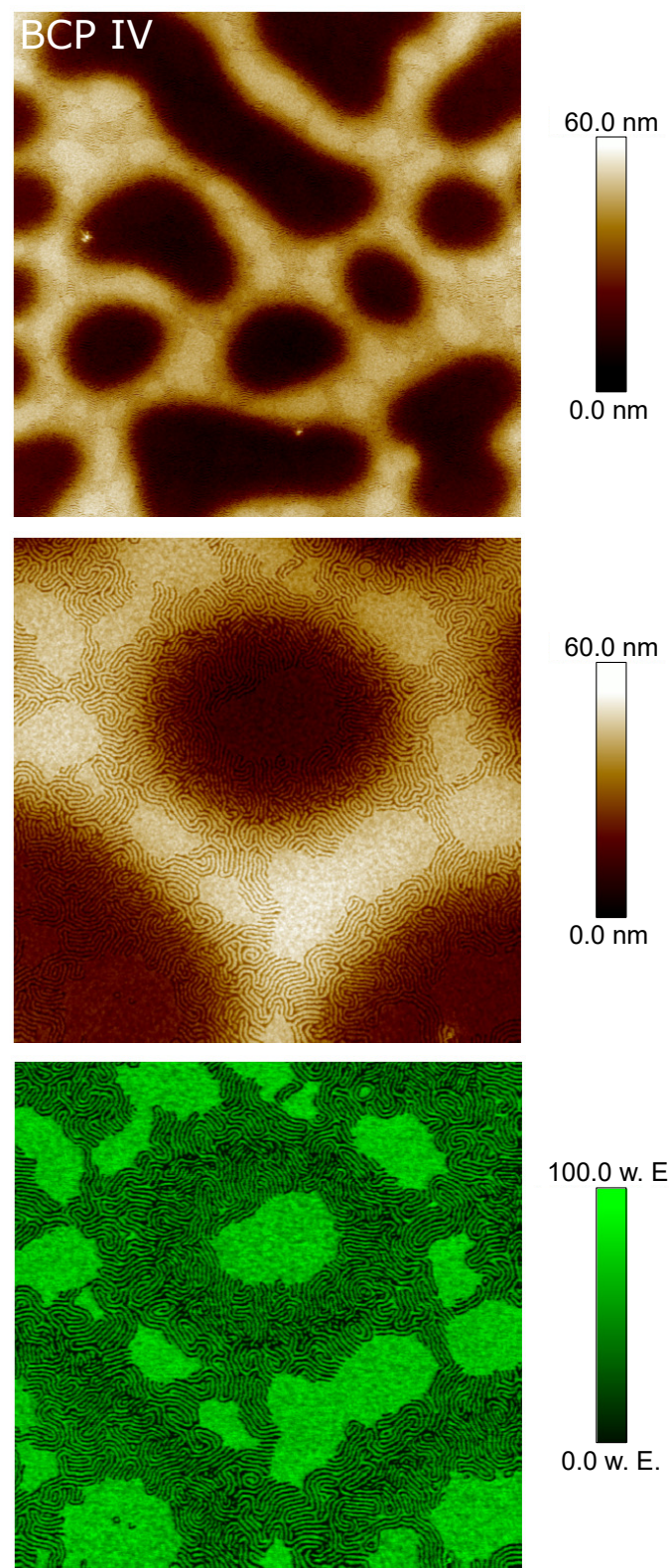

100.0 w. E.

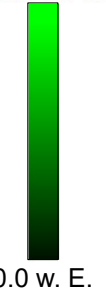

Abbildung 28: AFM-Aufnahmen des Höhenprofils, Ausschnitt: $16 \times 16 \mu \mathrm{m}$ (Oben), Ausschnitt: 5,3 × 5,3 $\mu \mathrm{m}$ (Mitte) und DMT-Modul, Ausschnitt: $5,3 \times 5,3 \mathrm{\mu m}$ (Unten) von BCP IV nach Tempern bei $180{ }^{\circ} \mathrm{C}$ für $24 \mathrm{~h}$. Auflösung: $512 \times 512$ Pixel. 
Die dreidimensionale Darstellung der Oberfläche von BCP II (Abbildung 29 zeigt deutlich, dass die Oberfläche der Ebenen und der Plateaus homogen ist. Die Plateaus weisen zudem die gleiche Höhe auf.

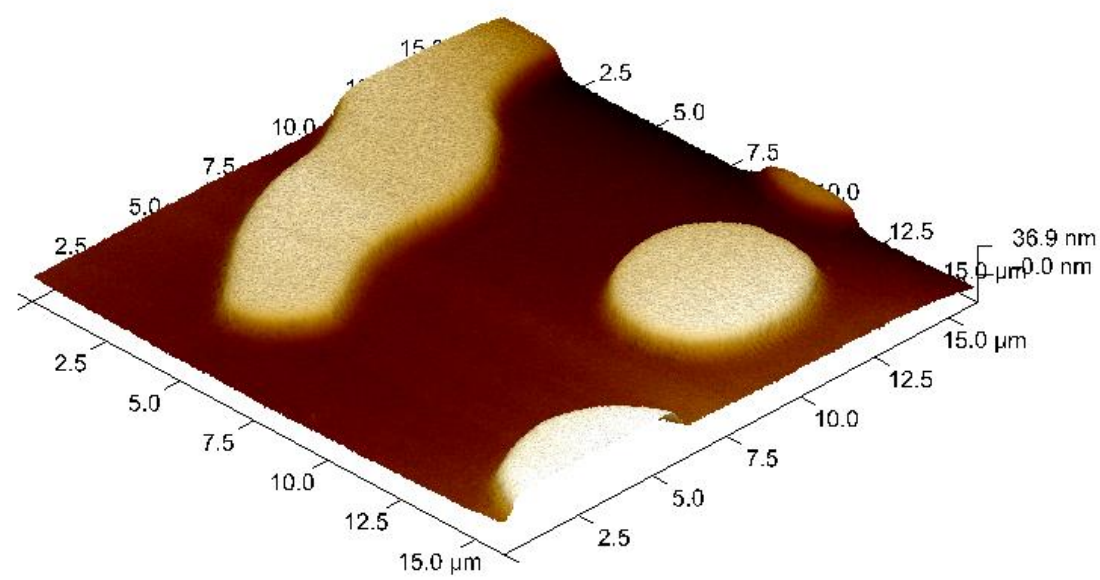

Abbildung 29: Dreidimensionale Darstellung der Oberfläche von BCP II aus der AFM-Aufnahme des Höhenprofils, Ausschnitt: $16 \times$ $16 \mu \mathrm{m}$.

Zwischen den drei Proben sind deutliche Unterschiede der Morphologie erkennbar. Bei BCP II liegen nur relativ kleine Bereiche mit vertikal angeordneten Lamellen vor, die sich stets an den Grenzen zwischen Plateau und Ebene befinden. Diese Bereiche sind bei BCP III bereits deutlich breiter, sie gehen jedoch immer noch von der Grenze zwischen den Ebenen aus. Bei BCP IV nehmen die senkrecht angeordneten Lamellen einen Großteil der Probenoberfläche ein. Die Grundstruktur der Ebenen-Plateau-Struktur ist jedoch immer noch vorhanden. Werden die Bereiche mit senkrecht angeordneten Lamellen genauer betrachtet, so fällt auf, dass die Defektdichte bei BCP II am geringsten ist und über BCP III zu BCP IV weiter ansteigt. Ein Grund dafür könnte die unterschiedliche Länge des PBA-Blockes und die damit einhergehende unterschiedliche Mobilität das Blockcopolymers sein. Ein längerer PBABlock führt zu einer geringeren Mobilität des Blockes. Somit ist die Mikrophasenseparation bei gleicher Temperatur in BCP IV langsamer als in BCP II und III. Dadurch würde die Temperzeit nicht ausreichen, um 
die lamellare Struktur umzuordnen und so Defekte in der Struktur zu reparieren. Dies würde sich mit den experimentellen Befunden decken, da die Struktur von BCP IV nach 24 stündigem Tempern in etwa mit der Struktur, die bei BCP III nach 30-60 minütigem Tempern vorliegt, übereinstimmt. Ob die Struktur von BCP IV sich durch längeres Tempern ändert und sich der Struktur von BCP II nähert, könnte durch weitere Experimente überprüft werden.

Zusätzlich zur qualitativen Analyse der AFM-Aufnahmen wurden einige Strukturparameter der Morphologie bestimmt, die einen quantitativen Vergleich der Blockcopolymere ermöglichen. Hieraus soll der Einfluss der unterschiedlich langen PBA-Blöcke deutlich werden. Die bestimmten Größen sind der Abstand der Lamellen in horizontaler Ausrichtung $d_{\mathrm{h}}$ und in vertikaler Ausrichtung $d_{\mathrm{v}}$ sowie die Breite des mikrophasenseparierten Bereiches $d_{\text {MPS }}$. Für die vertikal angeordneten Lamellen wurde zudem die Höhendifferenz der Mikrophasen $\Delta h$ und deren Breiten bei halber Höhendifferenz $d_{\mathrm{Azo}}$ und $d_{\mathrm{BA}}$ bestimmt, wie in Abbildung 30 gezeigt ist.

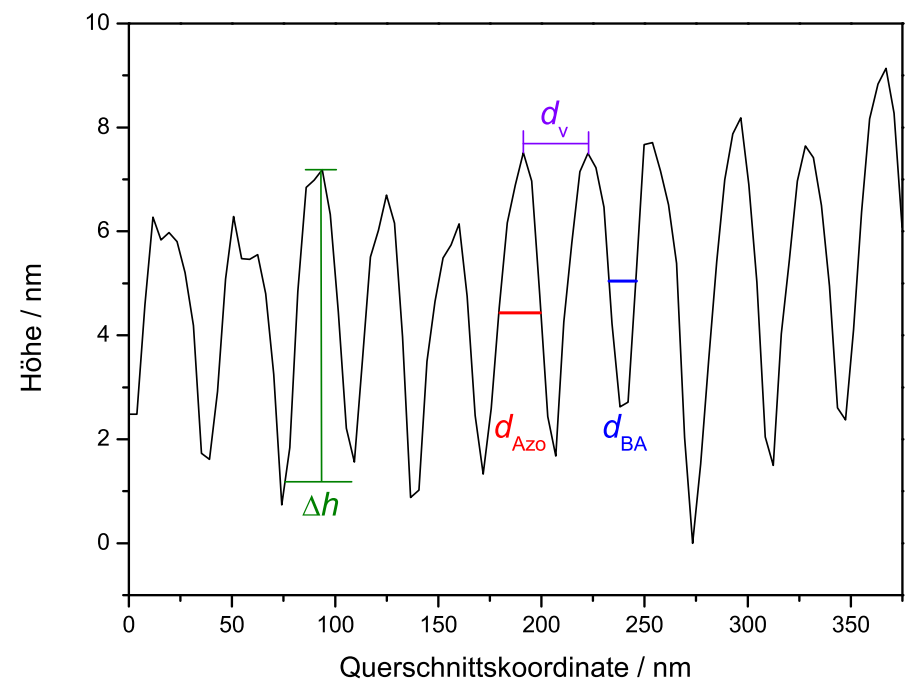

Abbildung 30: Höhenprofil eines Querschnitts durch vertikal angeordnete Lamellen in BCP II. Die Breite des PAzoPMA-Blockes $d_{\mathrm{Azo}}$ ist rot, die Breite des PBA-Blockes $d_{\mathrm{BA}}$ ist blau, die Höhendifferenz $\Delta h$ grün und der Lamellenabstand $d_{\mathrm{v}}$ lila markiert. 
Photoschaltbare Blockcopolymersysteme

Tabelle 9: Strukturparameter von BCP I-IV. Die mit * markierten Daten wurden zu Vergleichszwecken aus [36] übernommen.

\begin{tabular}{lcccc}
\hline & BCP I $^{*}$ & BCP II & BCP III & BCP IV \\
\hline$d_{\mathrm{h}} / \mathrm{nm}$ & $31,5 \pm 1,6$ & $33,0 \pm 1,8$ & $33,8 \pm 2,1$ & $34,3 \pm 2,1$ \\
$d_{\mathrm{V}} / \mathrm{nm}$ & $38,4 \pm 0,9$ & $38,8 \pm 5,5$ & $38,6 \pm 4,4$ & $45,9 \pm 5,4$ \\
$d_{\text {Azo }} / \mathrm{nm}$ & $22,3 \pm 2,7$ & $22,0 \pm 3,3$ & $22,5 \pm 2,8$ & $25,0 \pm 4,0$ \\
$d_{\mathrm{BA}} / \mathrm{nm}$ & $15,7 \pm 2,1$ & $17,0 \pm 3,8$ & $17,2 \pm 1,9$ & $20,6 \pm 3,4$ \\
$\Delta h / \mathrm{nm}$ & $4,2 \pm 1,2$ & $4,4 \pm 0,9$ & $4,3 \pm 0,9$ & $5,5 \pm 1,2$ \\
$d_{\text {MPS }} / \mathrm{nm}$ & $220 \pm 60$ & $300 \pm 30$ & $980 \pm 210$ & $1070 \pm 190$ \\
$n_{\text {BA }}$ & 230 & 249 & 330 & 416 \\
\hline
\end{tabular}

Die Strukturparameter sind zusammen mit der Anzahl der Monomereinheiten im PBA-Block $n_{\mathrm{BA}}$ in Tabelle 9 zusammengefasst. Zunächst ist $\mathrm{zu}$ erkennen, dass die Höhendifferenz der horizontalen Lamellen $d_{\mathrm{h}}$ sowie der Abstand der Lamellen in vertikaler Ausrichtung $d_{\mathrm{v}}$ mit zunehmender Länge des PBA-Blockes steigt. Durch die Verlängerung eines Blockes des Blockcopolymers steigt dessen Raumbedarf, sodass eine stärkere räumliche Ausdehnung der Phase in der mikrophasenseparierten Struktur folgt. Dadurch würde im vorliegenden Probensystem die Lamellenbreite ansteigen, was sich mit den experimentell gefundenen Ergebnissen deckt. Innerhalb einer Probe ist $d_{\mathrm{h}}$ stets kleiner als $d_{\mathrm{v}}$. Ein möglicher Grund dafür kann sein, dass die oberste Lamelle in der Probenoberfläche leicht einsinkt. Die Glasübergangstemperatur des PBA-Blockes ist bei Raumtemperatur bereits überschritten, sodass ein solches Phänomen möglich erscheint. Die Werte für $d_{\mathrm{Azo}}$ und $d_{\mathrm{BA}}$ steigen ebenfalls mit $n_{\mathrm{BA}}$ an. Die Verbreiterung der PBA-Phase kann durch die größere Anzahl an Monomereinheiten im PBA erklärt werden. Die Breite der PAzoPMA-Phase steigt ebenfalls an, jedoch weniger stark als die der PBA-Phase. Da sich das System nicht am SSL befindet, ist die Trennung der beiden Phasen nicht sehr scharf. Eine Verbreiterung einer Phase wirkt sich somit immer auch leicht auf die andere Phase aus. Die Höhendifferenz der Phasen ändert sich im Rahmen des Fehlerbereiches 
nicht. Große Unterschiede zeigen sich in der Breite des Bereiches, in dem vertikal angeordnete Lamellen vorliegen. Ist der Bereich bei BCP I noch sehr schmal und nur an der Grenze zwischen Plateau und Ebenen zu finden, so besteht bei BCP IV ein Großteil der Probenoberfläche aus senkrecht angeordneten Lamellen. Dies spiegelt sich auch in den bestimmten Werten für $d_{\text {MPS }}$ wider.

\subsection{Untersuchung der Mikrophasenseparation photoschaltbarer Blockcopolymer-Homopolymer-Blends}

Eine weitere Möglichkeit die Phasengrößen eines Blockcopolymers bei konstanter Morphologie zu modifizieren, besteht darin, das Blockcopolymer mit Homopolymer beider Blöcke zu mischen. ${ }^{93}$ Zur Herstellung der Komposite wurden zunächst Homopolymere der einzelnen Blöcke durch RAFT-Polymerisation synthetisiert, wie in Kapitel 6.9 beschrieben ist. Diese wurden durch GPC charakterisiert, die schmalen, monomodalen Molmassenverteilungen sind in Abbildung 31 gezeigt.

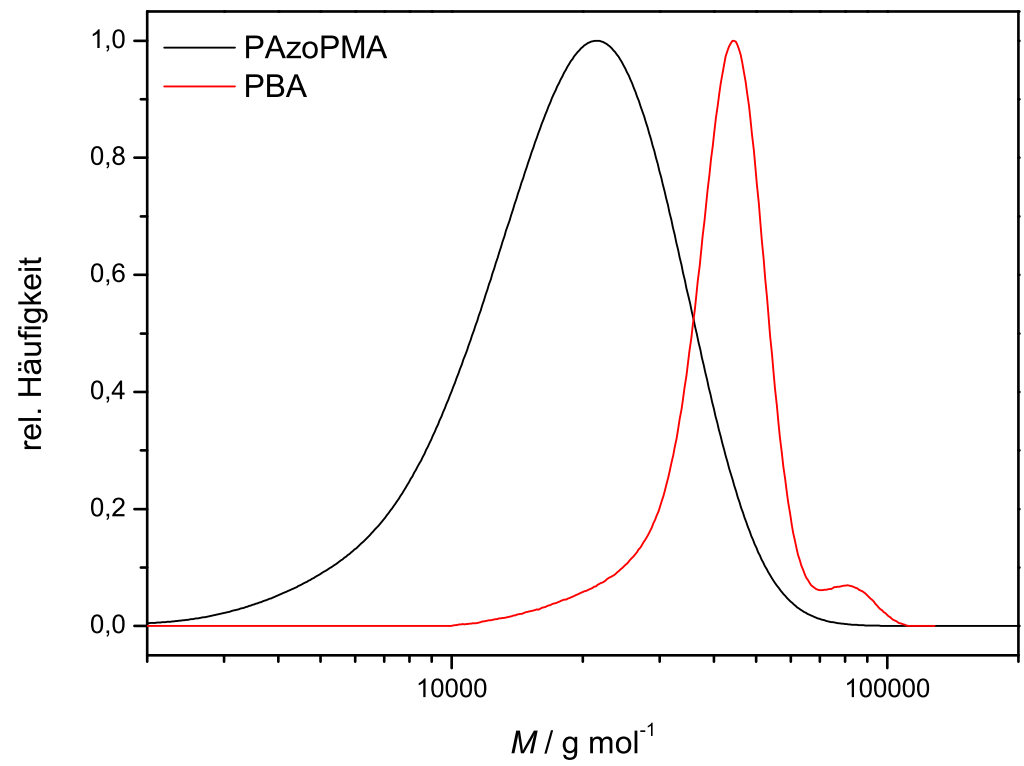

Abbildung 31: Aus dem GP-Chromatogramm berechnete normierte Molmassenverteilungen der Homopolymere PAzoPMA und PBA. 
Das verwendete PAzoPMA wies eine mittlere Molmasse von $M_{\mathrm{n}}=1,7 \cdot 10^{4} \mathrm{~g} \cdot \mathrm{mol}^{-1}$ und eine Dispersität von $Ð=1,38$ auf, für das PBA wurde eine mittlere Molmasse von $M_{\mathrm{n}}=3,1 \cdot 10^{4} \mathrm{~g} \cdot \mathrm{mol}^{-1}$ und eine Dispersität von $Ð=1,10$ ermittelt. Die Molmassen der Homopolymere entsprechen damit in etwa den Molmassen des Blockes in BCP I. Aus den Stoffmengenanteilen der Blöcke $\chi_{\mathrm{Azo}}$ und $\chi_{\mathrm{BA}}$ wurden zunächst die Massenanteile beider Blöcke $w_{\mathrm{Azo}}$ und $w_{\mathrm{BA}}$ berechnet.

$$
\begin{aligned}
w_{\mathrm{Azo}} & =\frac{\chi_{\mathrm{Azo}} \cdot M_{\mathrm{Azo}}}{\chi_{\mathrm{Azo}} \cdot M_{\mathrm{Azo}}+\chi_{\mathrm{BA}} \cdot M_{\mathrm{BA}}} \\
w_{\mathrm{BA}} & =1-w_{\mathrm{Azo}}
\end{aligned}
$$

Daraus ergeben sich die Gewichtsanteile $w_{\mathrm{Azo}}=0,354$ und $w_{\mathrm{BA}}=0,646$. Zur Herstellung der Blockcopolymer-Homopolymer-Komposite wurde BCP I mit 5 unterschiedlichen Gewichtsanteilen (10-50 Gew.-\%, BCP I+10 - BCP I+50) an Homopolymeren vermischt. Die Einwaagen sind in Kapitel 6.9 aufgeführt.

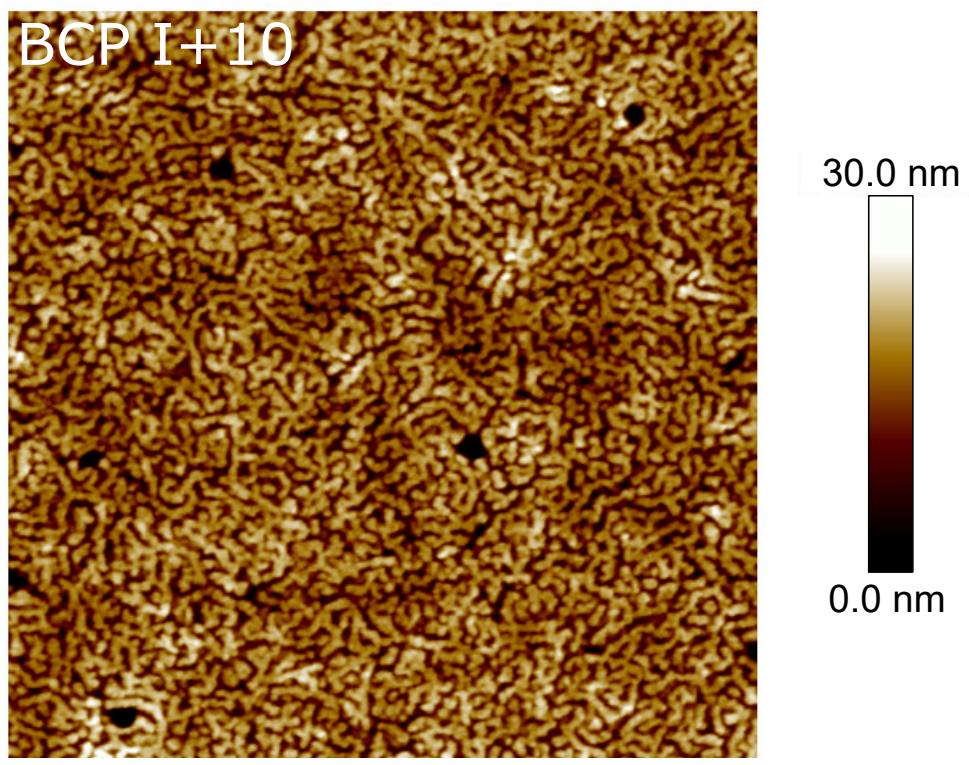

Abbildung 32: AFM-Aufnahmen des Höhenprofils von BCP $\mathrm{I}+10$ vor dem Tempern. Ausschnitt: 2,0 × 2,0 $\mu \mathrm{m}$, Auflösung: $512 \times 512 \mathrm{Pi}-$ xel. 
Das Verhältnis der Homopolymere entsprach dabei den Gewichtsanteilen der jeweiligen Blöcke im Blockcopolymer. Für alle Komposite wurden Proben durch Spincoaten auf Si-Substrate hergestellt und mit dem AFM untersucht. Das Höhenprofil von BCP I+10 vor dem Tempern ist in Abbildung 32 gezeigt. Die Grundstruktur gleicht dabei der von BCP I-BCP IV vor dem Tempern, sodass eine Mikrophasenseparation erkennbar ist, die sich keiner Morphologie zuordnen lässt. Es gibt keine Bereiche, in denen eine Ansammlung von Homopolymer erkennbar ist, sodass eine hinreichende Verteilung des Homopolymers im Blockcopolymer angenommen werden kann. Im Wesentlichen lassen sich zwei Unterschiede der Struktur von BCP I+10 zur Struktur von BCP I erkennen. Zum einen sind die Phasengrenzen zwischen den unterschiedlichen Blöcken des Blockcopolymers bereits etwas länger. Zum anderen ist der Abstand zwischen zwei Phasengrenzen größer, welches ein weiteres Indiz liefert, dass das Homopolymer in die Struktur des Blockcopolymers integriert ist.

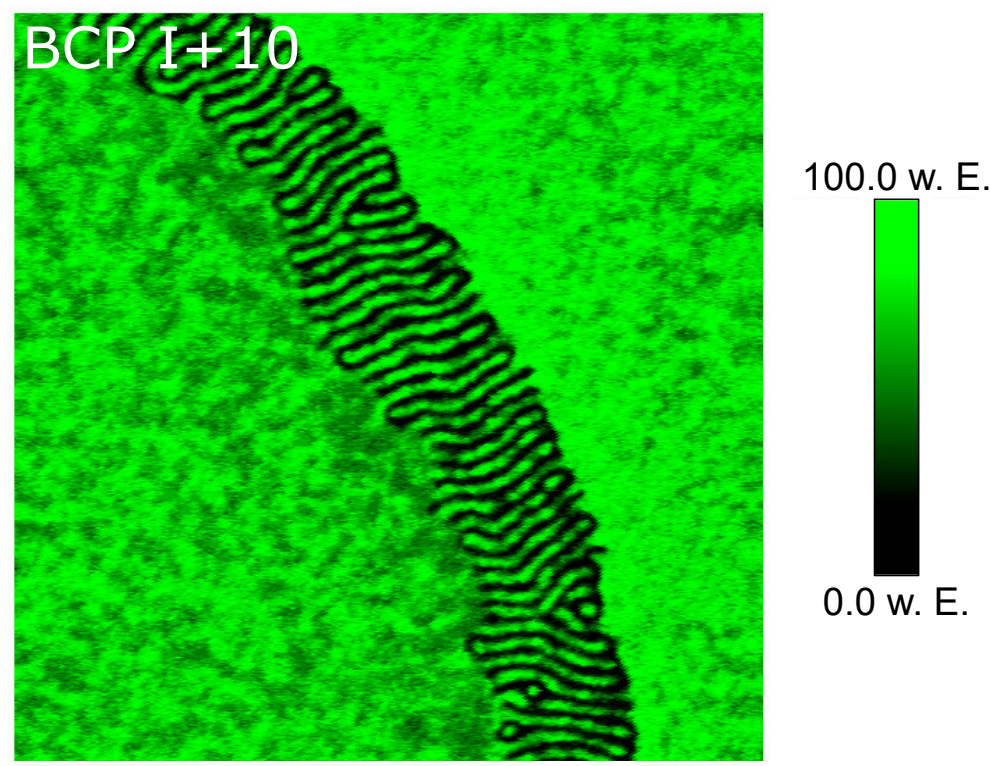

Abbildung 33: DMT-Modul der Oberfläche von BCP I+10 nach Tempern bei $180{ }^{\circ} \mathrm{C}$ für $24 \mathrm{~h}$, bestimmt durch AFM. Ausschnitt: 2,0 $\times$ 2,0 $\mathrm{mm}$, Auflösung: $512 \times 512$ Pixel. 
Nach dem Tempern bei $180{ }^{\circ} \mathrm{C}$ wurden die Proben auf Mikrophasenseparation sowie auf ihre mechanischen Eigenschaften mit dem AFM untersucht. Damit ist die Bestimmung von Strukturparametern und die Zuordnung der Blöcke möglich. Die Höhenprofile in verschiedenen Vergrößerungen von BCP I+10 bis BCP I+50 sind in den Abbildungen 34-38 gezeigt. Der DMT-Modul der Probenoberfläche von BCP I+10 ist als Beispiel in Abbildung $33 \mathrm{zu}$ sehen. Die Aufnahmen für die weiteren Blockcopolymere sind in Kapitel 7 in den Abbildungen 64-67 zu finden. Eine Betrachtung der Höhenprofile aller Proben zeigt, dass sie die selbe Morphologie aufweisen. Es handelt sich um eine lamellare Morphologie, wie sie auch bei BCP I vorliegt. ${ }^{36}$ Das System nimmt eine Struktur mit verschiedenen Ebenen an, die durch horizontal zur Oberfläche angeordneten Lamellen verursacht wird. An der Grenze der Ebenen liegen die Lamellen vertikal zur Substratoberfläche. Die Morphologie ändert sich durch die Zugabe von Homopolymer also nicht. Ein Vergleich des Höhenprofils und dem DMT-Modul von BCP I+10 macht deutlich, dass die Oberfläche der Ebenen aus hartem PAzoPMA bestehen. Der weichere PBA-Block ist nur in den Bereichen der vertikal zum Substrat angeordneten Lamellen zu beobachten. Die Anordnung der Blöcke ist daher analog zu BCP I. ${ }^{36}$ Es sind jedoch auch deutliche strukturelle Unterschiede zwischen dem Blockcopolymer und den Kompositen zu erkennen. Die Breite der Bereiche mit vertikal angeordneten Lamellen an der Grenze zwischen Ebene und Plateau steigt mit der Menge des zugegebenen Homopolymers signifikant an. Ebenfalls ist eine Abhängigkeit der Höhendifferenz zwischen Ebene und Plateaus sowie des Abstandes der vertikal angeordneten Lamellen von der zugegebenen Menge Homopolymer zu beobachten. Um diese Effekte quantifizieren zu können wurden der Abstand der Lamellen in horizontaler Ausrichtung $d_{\mathrm{h}}$ und in vertikaler Ausrichtung $d_{\mathrm{v}}$ sowie die Breite des Mikrophasenseparierten Bereiches $d_{\text {MPS }}$ bestimmt. Zudem wurde für die vertikal angeordneten Lamellen die Höhendifferenz der Mikrophasen $\Delta h$ und deren Breiten bei halber Höhendifferenz $d_{\mathrm{Azo}}$ und $d_{\mathrm{BA}}$ ermittelt. Diese Strukturparameter sind in Tabelle 10 zusammengefasst. 

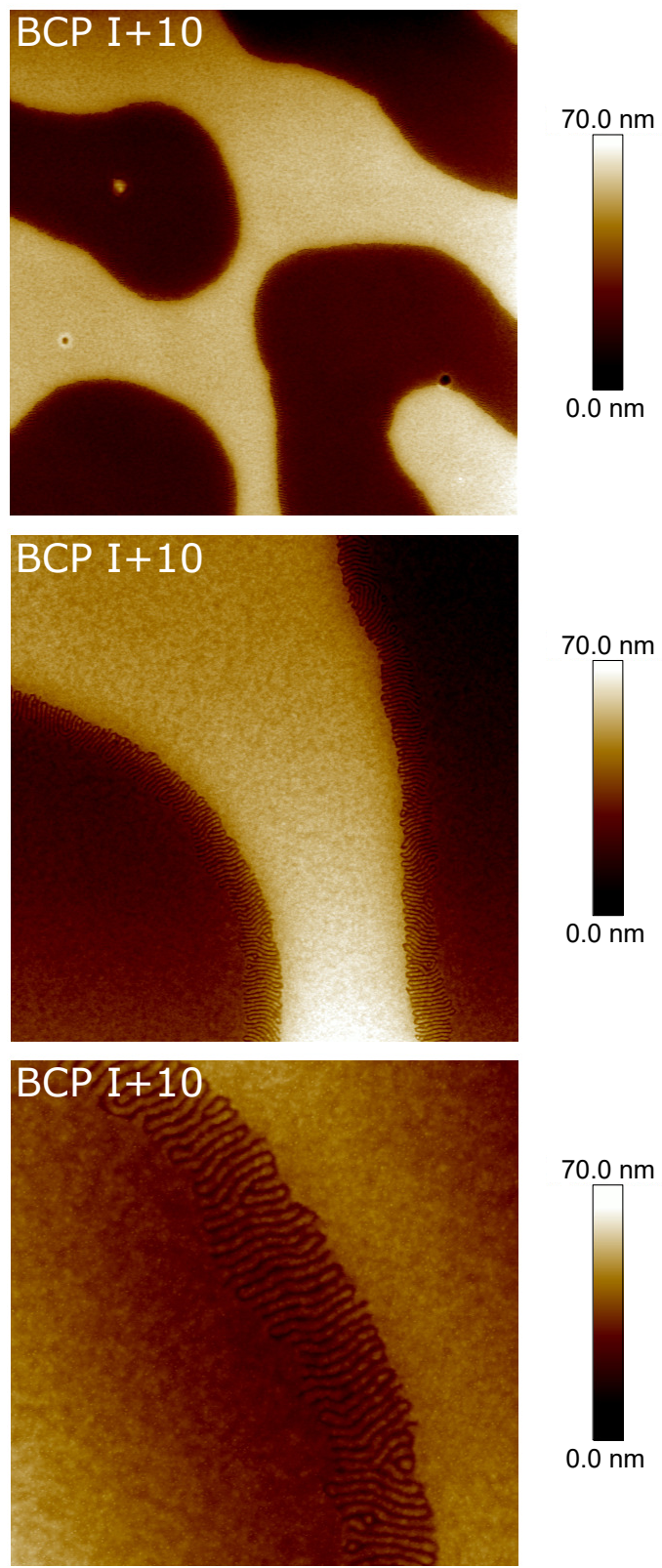

Abbildung 34: AFM-Aufnahmen des Höhenprofils von BCP I+10 nach Tempern bei $180{ }^{\circ} \mathrm{C}$ für $24 \mathrm{~h}$. Ausschnitt: $16 \times 16 \mu \mathrm{m}$ (Oben), $5,3 \times 5,3 \mu \mathrm{m}$ (Mitte) und 2,0 × 2,0 $\mathrm{mm}$ (Unten), Auflösung: $512 \times 512$ Pixel. 

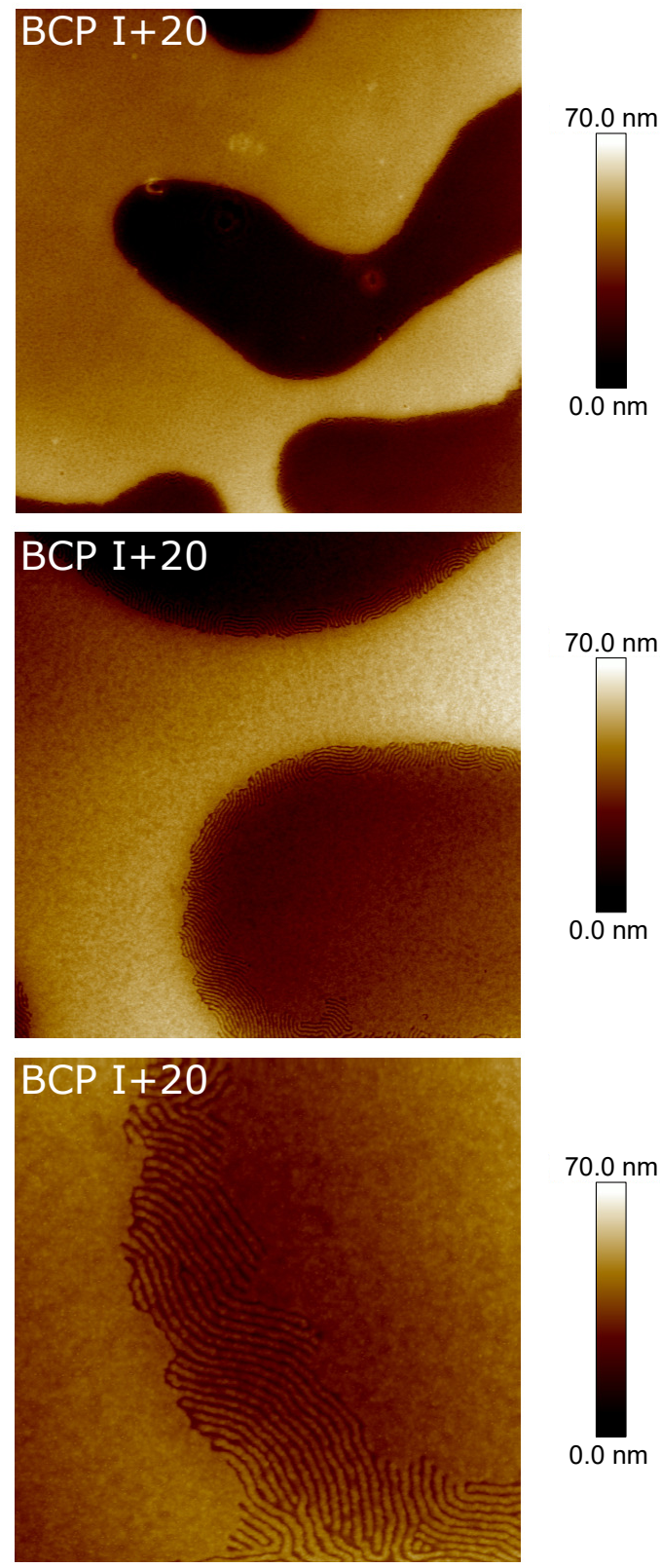

Abbildung 35: AFM-Aufnahmen des Höhenprofils von BCP I+20 nach Tempern bei $180{ }^{\circ} \mathrm{C}$ für $24 \mathrm{~h}$. Ausschnitt: $16 \times 16 \mu \mathrm{m}$ (Oben),

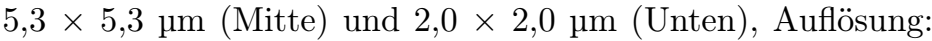
$512 \times 512$ Pixel. 

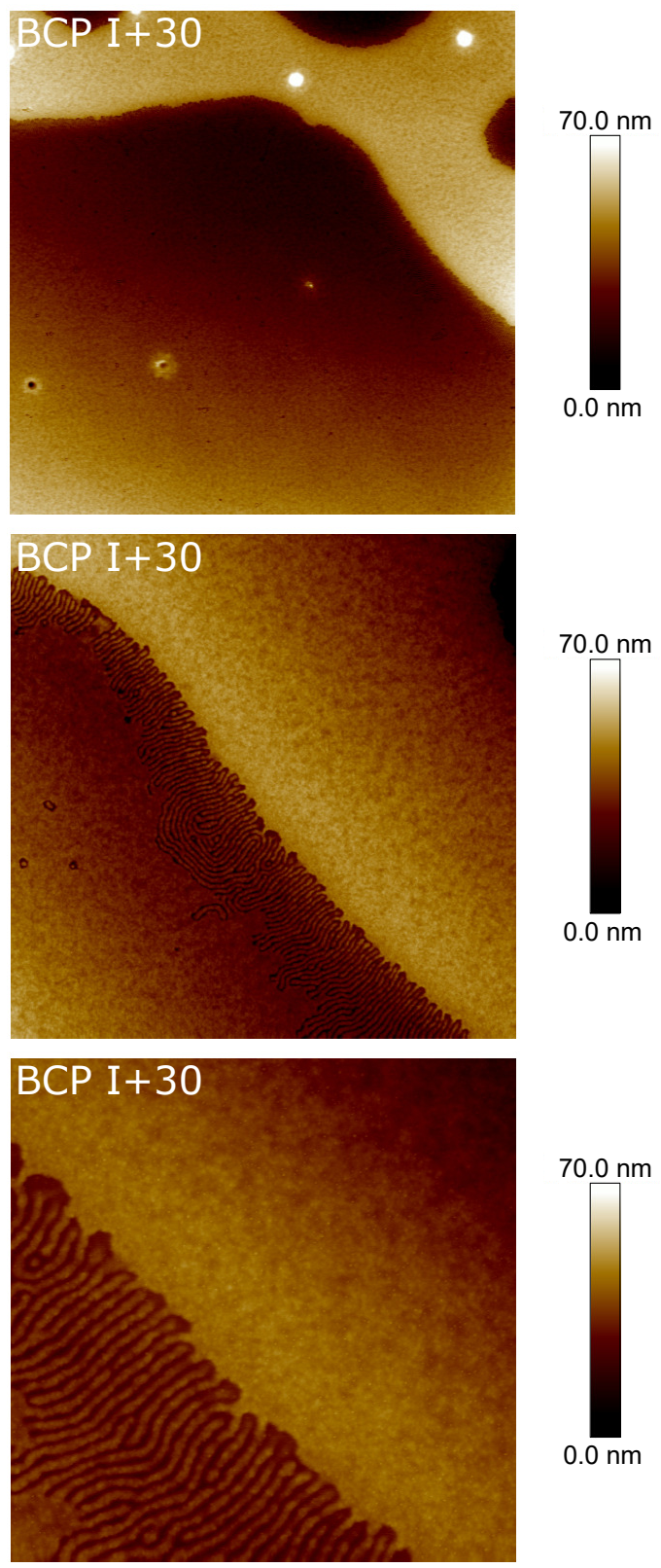

Abbildung 36: AFM-Aufnahmen des Höhenprofils von BCP I+30 nach Tempern bei $180{ }^{\circ} \mathrm{C}$ für $24 \mathrm{~h}$. Ausschnitt: $16 \times 16 \mu \mathrm{m}$ (Oben), $5,3 \times 5,3 \mu \mathrm{m}$ (Mitte) und 2,0 × 2,0 $\mu \mathrm{m}$ (Unten), Auflösung: $512 \times 512$ Pixel. 

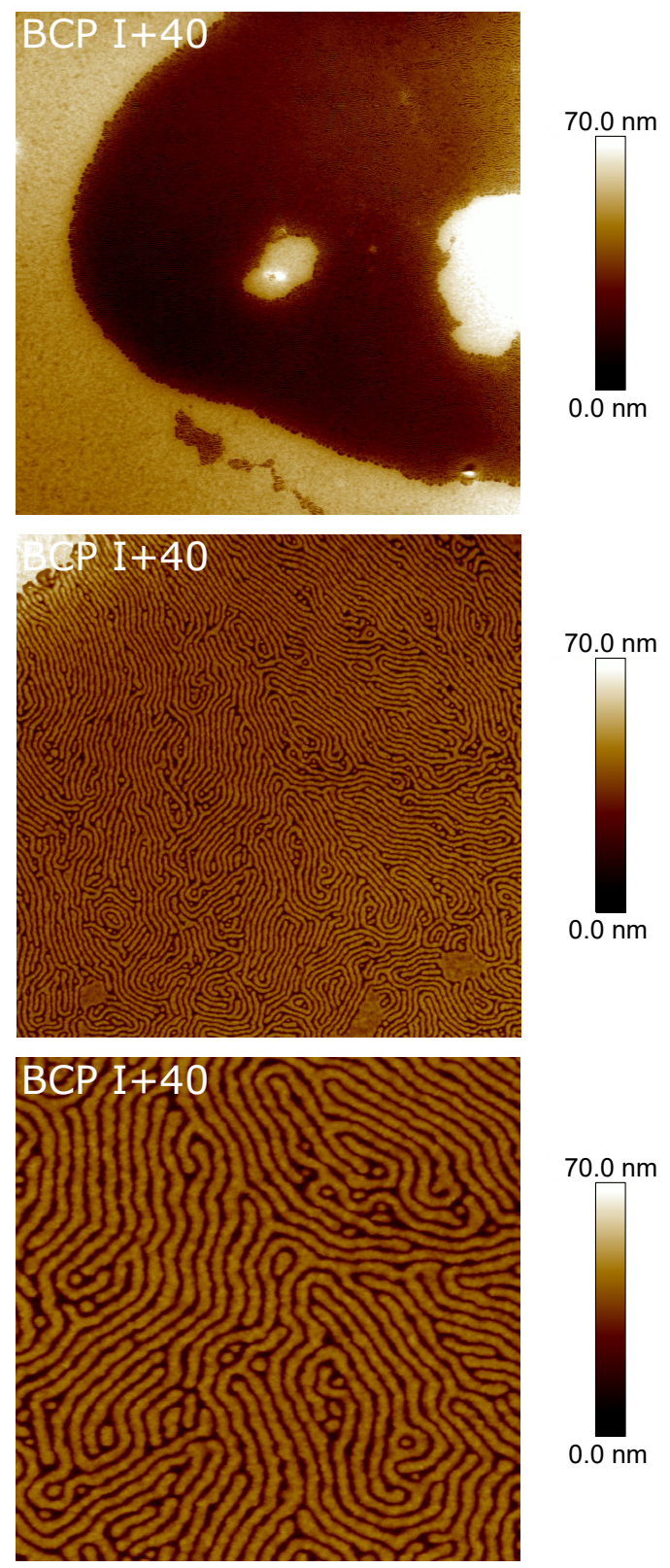

Abbildung 37: AFM-Aufnahmen des Höhenprofils von BCP I +40 nach Tempern bei $180{ }^{\circ} \mathrm{C}$ für $24 \mathrm{~h}$. Ausschnitt: $16 \times 16 \mu \mathrm{m}$ (Oben), $5,3 \times 5,3 \mu \mathrm{m}$ (Mitte) und 2,0 × 2,0 $\mu \mathrm{m}$ (Unten), Auflösung: $512 \times 512$ Pixel. 

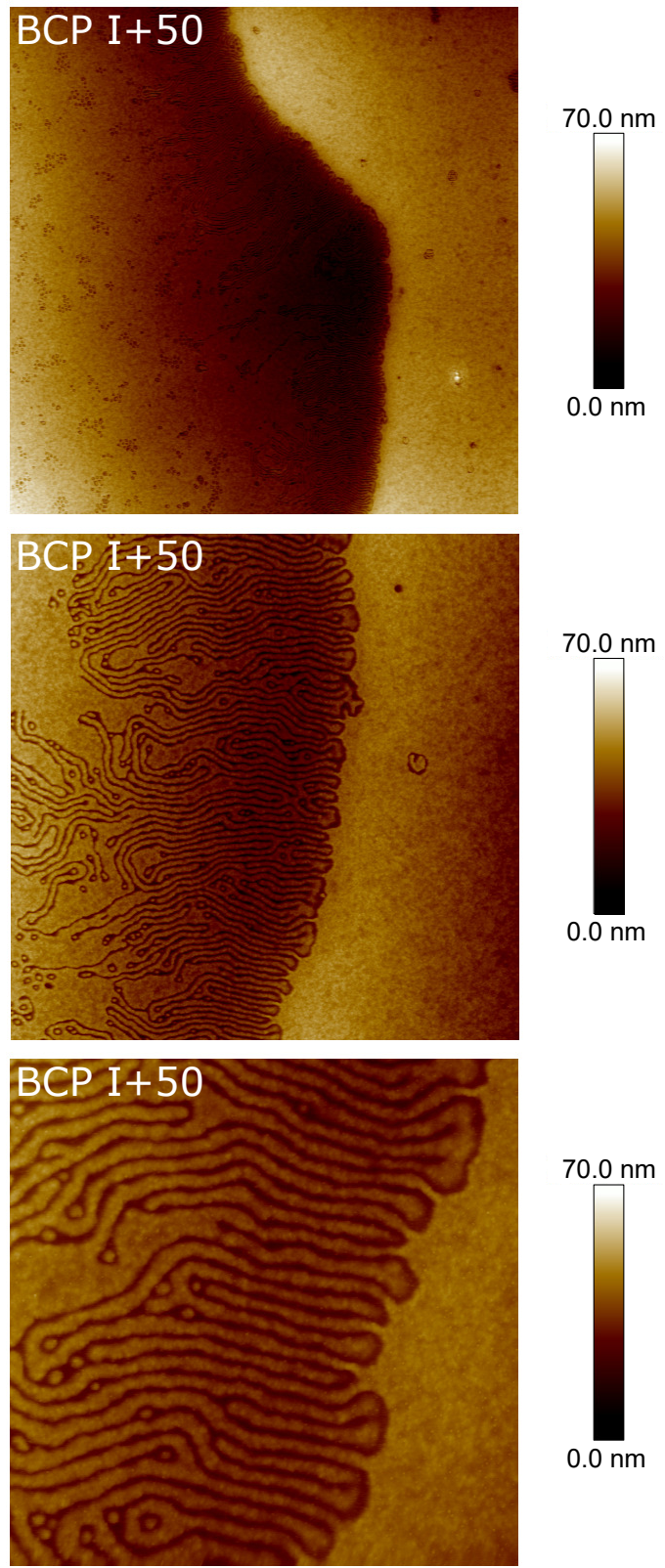

Abbildung 38: AFM-Aufnahmen des Höhenprofils von BCP I+50 nach Tempern bei $180{ }^{\circ} \mathrm{C}$ für $24 \mathrm{~h}$. Ausschnitt: $16 \times 16 \mu \mathrm{m}$ (Oben), $5,3 \times 5,3 \mu \mathrm{m}$ (Mitte) und 2,0 × 2,0 $\mathrm{mm}$ (Unten), Auflösung: $512 \times 512$ Pixel. 
Die Strukturparameter von BCP I+20 unterscheiden sich im Fehlerbereich nicht von den Strukturparametern von BCP I. Es ist davon auszugehen, dass das Homopolymer in diesem Fall nicht in die Struktur des Blockcopolymers eingelagert wurde. Beim weiteren Vergleich der Blockcopolymer-Homopolymer-Komposite wird BCP I+20 deshalb nicht betrachtet.

Tabelle 10: Strukturparameter von BCP I+10-BCP I+50. Die mit * markierten Daten wurden zu Vergleichszwecken aus [36] übernommen. Alle Größen sind in nm angegeben.

\begin{tabular}{lcccccc}
\hline & BCP I $^{*}$ & BCP I+10 & BCP I+20 & BCP I+30 & BCP I+40 & BCP I+50 \\
\hline$d_{\mathrm{h}}$ & $31,5 \pm 1,6$ & $34,9 \pm 1,4$ & $31,2 \pm 1,6$ & $38,5 \pm 2,4$ & $41,8 \pm 1,9$ & $45,0 \pm 2,5$ \\
$d_{\mathrm{V}}$ & $38,4 \pm 0,9$ & $47,6 \pm 5,6$ & $41,2 \pm 0,9$ & $53,2 \pm 6,3$ & $61,9 \pm 6,8$ & $73,0 \pm 9,2$ \\
$d_{\text {Azo }}$ & $22,3 \pm 2,7$ & $28,1 \pm 3,5$ & $23,9 \pm 2,0$ & $30,4 \pm 3,2$ & $36,2 \pm 4,7$ & $44,6 \pm 5,4$ \\
$d_{\text {BA }}$ & $15,7 \pm 2,1$ & $20,0 \pm 3,3$ & $18,3 \pm 2,5$ & $23,0 \pm 3,0$ & $25,5 \pm 3,6$ & $27,1 \pm 4,9$ \\
$\Delta h$ & $4,2 \pm 1,2$ & $9,1 \pm 1,9$ & $4,8 \pm 1,5$ & $9,9 \pm 1,8$ & $10,3 \pm 1,5$ & $11,4 \pm 1,7$ \\
$d_{\text {MPS }}$ & $220 \pm 60$ & $330 \pm 30$ & $250 \pm 40$ & $750 \pm 180$ & $1800 \pm 330$ & $2900 \pm 940$ \\
\hline
\end{tabular}

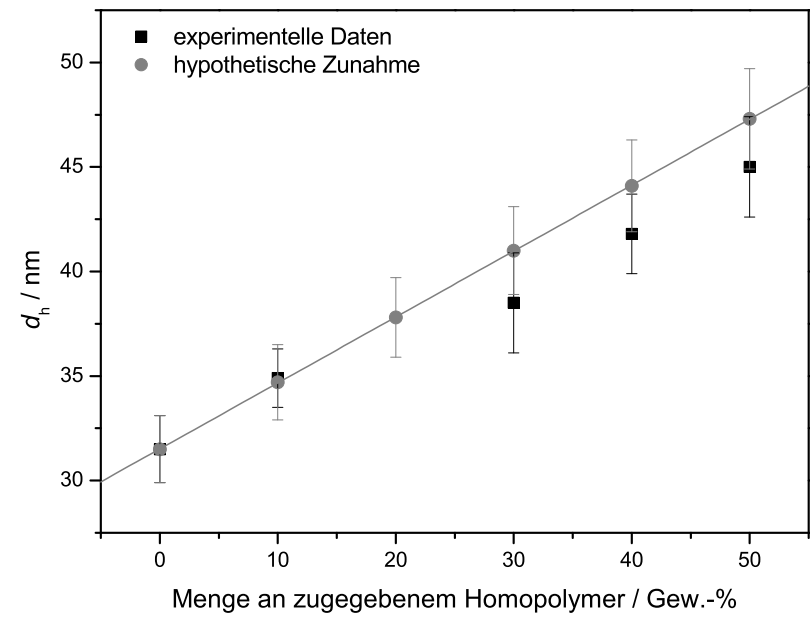

Abbildung 39: Auftragung des horizontalen Lamellenabstandes von BCP I sowie BCP I+10-BCP I+50 gegen die Menge an zugegebenem Homopolymer, sowie berechnete hypothetische Zunahme des horizontalen Lamellenabstandes, ausgehend vom Wert von BCP I. 

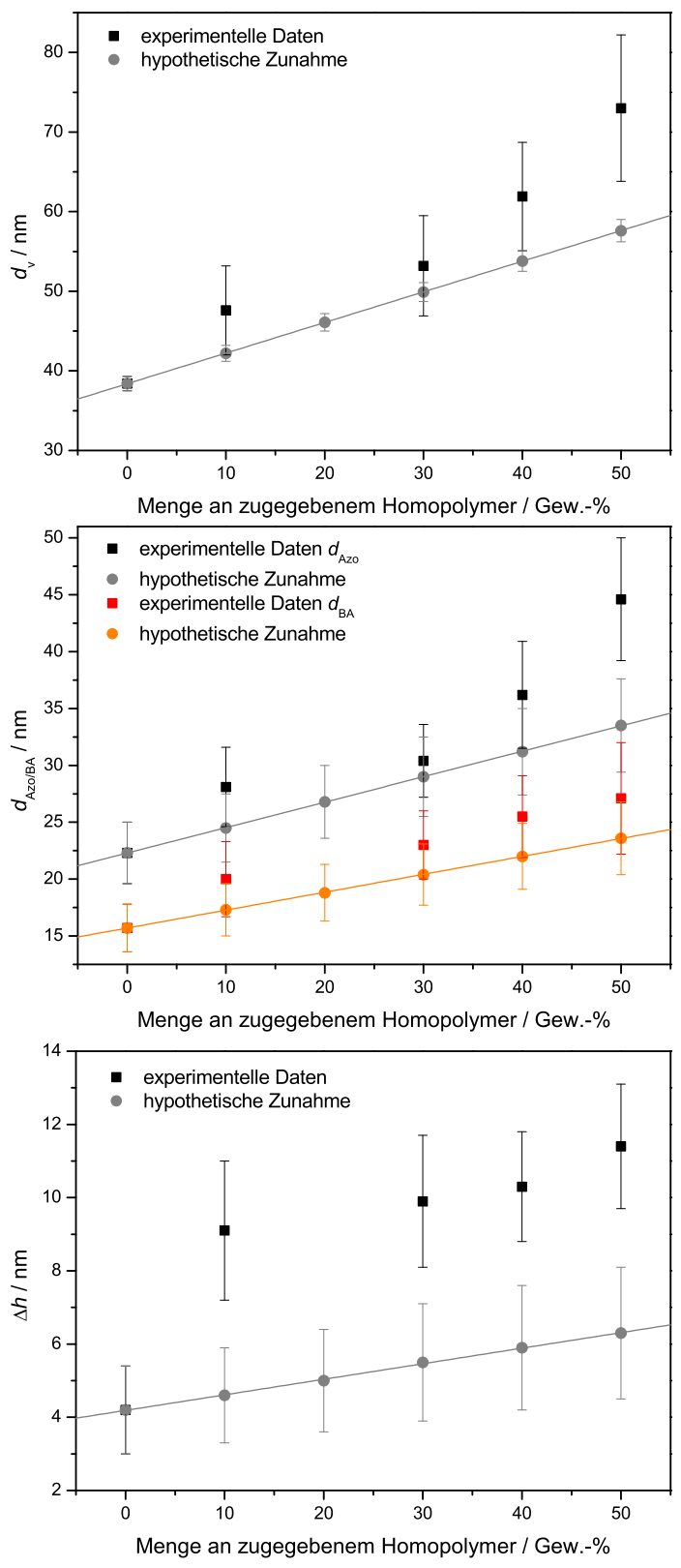

Abbildung 40: Auftragung der experimentell ermittelten Strukturparameter von BCP I sowie BCP I+10-50 gegen die Menge an zugegebenem Homopolymer, sowie berechnete hypothetische Zunahme der Strukturparameter, ausgehend vom Wert von BCP I. 
Für alle Strukturparameter ist ohne Ausnahme ein Anstieg des Parameters mit der Menge des zugegebenen Homopolymers zu erkennen. Im Folgenden sollen die Änderungen aller Größen, sowie mögliche Gründe dafür diskutiert werden.

Die Höhe der horizontal angeordneten Lamellen $d_{\mathrm{h}}$ steigt von $31,5 \mathrm{~nm}$ in BCP I auf 45,0 nm in BCP I+50 an. Mit Ausnahme von BCP I+10, bei dem die Höhendifferenz der extrapolierten Zunahme entspricht, liegen die Werte für $d_{\mathrm{h}}$ unterhalb der Extrapolation. Für BCP I+30 bis BCP I+50 ist eine lineare Zunahme zu erkennen. Ein Grund für dieses Verhalten kann ein Einsinken der horizontal angeordneten Lamellen sein, wie bereits in Kapitel 3.3.2 diskutiert wurde. Je dicker eine Lamelle ist, desto größer ist ihre Masse und desto stärker die auf sie wirkende Schwerkraft, die zum Einsinken führt. Dies kann eine mögliche Begründung dafür sein, dass die bestimmten Lamellendicken kleiner als die Extrapolation sind. Da die Fehlerbereiche der Parameter sich jedoch auch mit denen der Extrapolation überlappen, kann der Anstieg der Lamellendicke als erwartungsgemäß bezeichnet werden.

Die Dicke der vertikal angeordneten Lamellen $d_{\mathrm{v}}$ steigt von $38,4 \mathrm{~nm}$ in BCP I auf 73,0 nm in BCP I+50 an. Dabei liegen die bestimmten Strukturparameter stets über den durch Extrapolation vorhergesagten Werten, steigen jedoch auch mit der Menge an zugegebenem Homopolymer an. Mögliche Gründe für dieses Verhalten können in den Eigenschaften einer Blockcopolymer-Homopolymer-Mischung gefunden werden. Die Dichte von binären Mischungen, bei denen beide Komponenten eine ähnliche Struktur aufweisen, ist stets geringer als die gewichtete Summe der beiden einzelnen Komponenten. ${ }^{94}$ Daher sollte die Dichte einer Mischung eines Blockes des Blockcopolymers mit Homopolymer ebenfalls geringer sein, als die Dichte der Komponenten. Bei einem mikrophasenseparierten Blockcopolymer befinden sich die Vernüpfungspunkte der beiden Blöcke in der Phasengrenzfläche, die Ketten sind in der Nähe der Grenzfläche zur Energieminimierung gestreckt. ${ }^{79,95}$ Dies hat zur Folge, dass das Packen der Ketten in diesem Bereich besonders effizient und dicht erfolgen kann und die Dichte des Blockcopo- 
lymers demnach größer ist, als in einem Homopolymer. Dementsprechend würde eine Zugabe von Homopolymer zu einem Blockcopolymer zu einer Verringerung der Dichte des Systems führen. Des Weiteren ist anzunehmen, dass sich Homopolymer zwar bevorzugt, aber nicht ausschließlich im Block des selben Monomers im Blockcopolymer anordnet. Da das Homopolymer mit dem jeweils anderen Block schlecht mischbar ist, kann auch dies zu einer Verringerung der Dichte der Mischung führen, da weniger attraktive und mehr repulsive Wechselwirkungen vorliegen würden.

Die genannten Gründen legen nahe, dass im untersuchten System von einer Verringerung der Dichte des Blockcopolymers durch die Zugabe von Homopolymer auszugehen ist. Dadurch würde die Breite der Lamellen stärker ansteigen, als es die Extrapolation vorhersagt, welches sich mit den experimentellen Befunden deckt.

Ähnliche Trends zeigen sich auch bei den Breiten der einzelnen Blöcke $d_{\mathrm{Azo}}$ und $d_{\mathrm{BA}}$. Die Breite der PAzoPMA-Phase steigt von $22,3 \mathrm{~nm}$ in BCP I auf 44,6 nm in BCP I+50, die Breite der BA-Phase von 15,7 nm in BCP I auf 27,1 nm in BCP I+50 mit der Menge an zugegebenem Homopolymer an. Dabei liegen die Werte der Strukturparameter stets über denen der Extrapolation. Dies kann analog zur Breite der vertikalen Lamellen auf die Änderung der Dichte zurückgeführt werden.

Die Abhängigkeit der Höhendifferenz zwischen PAzoPMA-Phase und PBA-Phase im Bereich der vertikal angeordneten Lamellen $\Delta h$ von der Menge an zugegebenem Homopolymer weicht im Verhalten von dem der anderen Strukturparameter ab. Von BCP I zu BCP I+10 zeigt sich bereits ein deutlicher Anstieg von 4,2 nm auf 9,1 nm. Von dort an steigt der Wert der Höhendifferenz zu BCP I+50 nur noch leicht auf 11,4 nm an. Dieser Anstieg verläuft parallel zur Extrapolation. Die Oberfläche eines mikrophasenseparierten Blockcopolymers wird im Wesentlichen durch die Wechselwirkung der Blöcke, sowie deren Interaktion mit dem Material über der Probe (hier: Luft) bestimmt. ${ }^{96,97}$ Beim Tempern ordnet sich das Blockcopolymer bzw. die Blockcopolymermischung so an, dass die Oberflächenenergie minimal wird. Bei einem reinen Blockcopoly- 
mer wie BCP I ist die Möglichkeit der Umorientierung geringer, da die Verknüpfungspunkte der Blöcke in der Phasengrenzfläche fixiert sind. In den Blockcopolymer-Homopolymer-Mischungen kann sich das Homopolymer frei im Blockcopolymer anordnen, sodass der energetisch günstigste Zustand erreicht wird. Eine Entmischung von Homopolymer und Blockcopolymer ist entropisch ungünstig, sodass die Struktur der Mikrophasenseparation erhalten bleibt. Das Homopolymer ordnet sich jedoch so an, dass die Energie der Oberflächenstruktur minimiert wird. Das könnte im Falle von BCP I+10 bis BCP I+50 dazu führen, dass die Höhendifferenzen zwischen PAzoPMA und PBA-Block zunehmen. Demnach würde PAzoPMA die geringer Oberflächenenergie an der Grenze zu Luft aufweisen. Dies deckt sich mit den zuvor vorgestellten experimentellen Befunden, bei denen die Oberfläche der horizontalen Lamellen aus PAzoPMA besteht.

Die Breite $d_{\text {MPS }}$ des Bereiches, in dem vertikale Lamellen vorliegen, ist ebenfalls stark von der Menge des zugegebenen Homopolymers abhängig. In BCP I ist dieser Bereich durchschnittlich $220 \mathrm{~nm}$ breit, bei BCP I+50 erstreckt er sich über durchschnittlich 2,9 $\mu \mathrm{m}$. BCP I bevorzugt eine horizontale Anordnung der Lamellen. Die vertikal ausgerichteten Lamellen treten in den Bereichen auf, in denen bei der Struktur der horizontalen Lamellen der PBA-Block an der Oberfläche wäre. Durch die vertikalen Lamellen kann der Anteil an PAzoPMA an der Oberfläche weiter erhöht werden. ${ }^{36}$ Wie aus den experimentellen Befunden deutlich wird, verbreitert die Zugabe der Homopolymere den Bereich der vertikal angeordneten Lamellen beträchtlich. Die Gründe für dieses Verhalten können durch eine Betrachtung des Temperprozesses sowie der Oberflächenenergie gefunden werden. Wie durch die AFM-Aufnahmen der ungetemperten Blockcopolymere (Abb. 32) deutlich wird, liegt vor dem Tempern eine Struktur vor, die bereits Anfänge einer Mikrophasenseparation zeigt, jedoch noch keine klare Morphologie aufweist. Während des Temperprozesses erfolgt dann die neue Anordnung hin zu den bekannten Gleichgewichtsstrukturen. Da die Anordnung in horizontalen Lamellen energetisch bevorzugt ist, strebt das 
System diese Struktur an. Lamellen, welche andere Anordnung haben, werden folglich eine Umorientierung anstreben, solange die Gesamtenergie dabei weiter minimiert wird. Wird kein Homopolymer zugegeben, ist die Umorientierung die einzige Möglichkeit die Oberflächenenergie des Systems zu ändern. Zusätzliches Homopolymer im System könnte jedoch eine Alternative liefern, um die Energie zu optimieren. Die Oberfläche der vertikal angeordnete Lamellen könnte wie bereits beschrieben durch das Homopolymer stabilisiert werden, wodurch der Drang des Systems, horizontal ausgerichtete Lamellen auszubilden, sinken würde.

In realen mikrophasenseparierten Strukturen treten stets Defekte auf. Ein Defekt ist dabei eine Abweichung von der Idealstruktur. Diese können den drei Defektklassen Sackgasse, Kreuzung und Bogen zugeordnet werden, welche in Abb. 41 gezeigt sind. ${ }^{98}$ Bei einer Sackgasse endet eine Lamelle, wodurch eine stark gekrümmte Phasengrenzfläche entsteht. An Kreuzungen laufen mehrere Lamellen zusammen. Dies geschieht häufig in Gebieten, in denen lamellare Bereiche mit unterschiedlicher Ausrichtung aufeinandertreffen. Bei Bögen durchläuft eine Lamelle eine Kurve von bis zu $180^{\circ}$. Auch hier ergibt sich eine ungünstig gekrümmte Phasengrenzfläche. Allgemein ist anzumerken, dass jeder Defekt weitere Defekte in der Probe verursachen kann. Eine Sackgasse kann beispielsweise einen Bogen verursachen.

Eine quantitative Analyse der Defekte und der Defektdichte ist für die vorliegenden Proben aus einigen Gründen nicht sinnvoll. Die Defektdichte gibt die Anzahl der Defekte pro Fläche an und ist somit nicht geeignet mikrophasenseparierte Strukturen zu vergleichen, bei denen sich die Breite der Wiederholeinheit ändert. Zudem unterscheidet sich die Breite des Bereiches, in dem die vertikalen Lamellen vorliegen, für die untersuchten Proben stark. In einem schmaleren Bereich ist das Ausheilen eines Defektes wesentlich einfacher, da sich weniger Polymer neu anordnen muss um den Defekt zu beheben. Dies zeigt sich auch durch den optischen Eindruck beim Betrachten der Höhenprofile der Blockcopolymer-Homopolymer-Komposite. Die Struktur von 
BCP I+10 zeigt nur sehr wenige Defekte, während die Strukturen mit zunehmendem Anteil an zugegebenem Homopolymer immer größere Unordnung zeigen. Dafür können im wesentlichen zwei Gründe angeführt werden. Zum einen ist eine Stabilisierung von Defektstellen durch das Homopolymer möglich, sodass das Ausheilen der Defekte nur noch eine kleinere Verringerung der Gesamtenergie zur Folge hätte. Zum anderen ist, wie bereits ausgeführt wurde, durch die zunehmende Breite des Bereiches mit vertikalen Lamellen das Ausheilen von Defekten zunehmend schwieriger, da sich mehr Polymer dafür umordnen müsste.

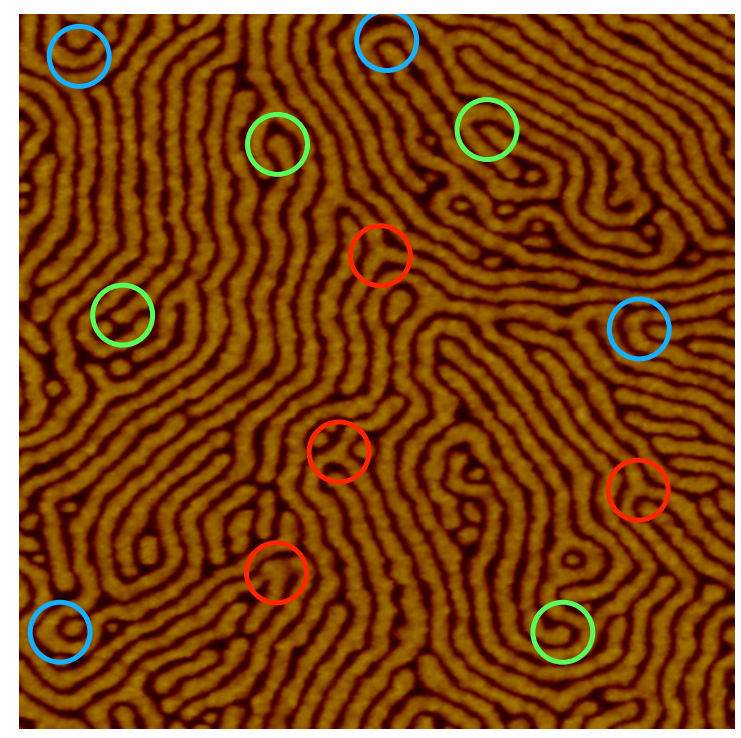

Abbildung 41: Beispiele für auftretende Defekte im Höhenprofil von BCP I +40 . Ausschnitt: $2,0 \times 2,0 \quad \mu \mathrm{m}$, Auflösung: $512 \times 512$ Pixel. Die verschiedenen Defektklassen sind Sackgassen (grün), Kreuzungen (rot) und Bögen (blau). Es sind nicht alle auftretenden Defekte markiert. 


\subsection{Photospaltbare Blockcopolymeraggregate}

Neben der Untersuchung azobenzolhaltiger Blockcopolymere in fester Phase ist deren Betrachtung in Lösung interessant. Durch das Isomerisieren der Azobenzol-Gruppe ändert sich die Polarität und somit auch die Löslichkeit des Blockes drastisch. In einer vorangegangenen Arbeit konnte gezeigt werden, dass Blockcopolymere aus PAzoPMA und BA in Toluol in Lösung durch Bestrahlung mit UV-Licht reversibel aggregieren. ${ }^{36}$ Außerdem zeigte ein Blockcopolymer aus PAzoPMA und Poly-(2-(Dimethylamino)ethylmethacrylat) (PDMAEMA) entgegengesetzte Eigenschaften. Das Blockcopolymer konnte unter UVBestrahlung in Propylencarbonat gelöst werden, durch Erhitzen bildeten sich kleinere Aggregate mit einem hydrodynamischen Radius von knapp $100 \mathrm{~nm}$. Ausgehend von diesem System sollen lichtspaltbare Blockcopolymeraggregate entwickelt werden, die mit kleinen Molekülen befüllt werden können, die lichtinduziert freigesetzt werden. Als kleines Molekül wurde in dieser Arbeit der Farbstoff Kristallviolett (Abb. 42) verwendet.<smiles>CN(C)c1ccc(C(=C2C=CC(=[N+](C)C)C=C2)c2ccc(N(C)C)cc2)cc1</smiles>

Abbildung 42: Struktur von Kristallviolett.

Dafür sind zwei Gründe maßgeblich. Zum einen weist Kristallviolett eine Bande bei etwa $590 \mathrm{~nm}$ mit einem hohen Extinktionskoeffizienten auf, sodass der Erfolg der Herstellung durch UV/Vis-Spektroskopie überprüft werden kann. Zudem weist es keine Banden auf, die mit denen des Blockcopolymers bei 350 und $440 \mathrm{~nm}$ überlagern. Zum anderen hat Kristallviolett in etwa die Größe vieler gängiger Medikamente, was 
im Hinblick auf die Entwicklung von gefüllten Blockcopolymeraggregaten als neue Strategie zur Wirkstoffverabreichung wichtig ist.

Zur Herstellung der gefüllten, photoschaltbaren Blockcopolymeraggregate wurde PAzoPMA- $b$-PDMAEMA (BCP V) mit einer durchschnittlichen molaren Masse von $M_{\mathrm{n}}=4,1 \cdot 10^{4} \mathrm{~g} \cdot \mathrm{mol}^{-1}$, einer Dispersität von $Ð=1,40$ und einem Stoffmengenanteil an AzoPMA von $\chi_{\text {Azo }}=0,27$ verwendet. Propylencarbonat wurde als Lösungsmittel eingesetzt. Der PDMAEMA-Block ist dabei im gewählten Zustand stets gut löslich. Der PAzoPMA-Block weist in der cis-Konfiguration ebenfalls eine gute Löslichkeit auf, in der trans-Konfiguration hingegen ist er schlecht löslich. BCP V wurde unter Bestrahlung mit UV-Licht in Propylencarbonat gelöst und die Lösung anschließend auf $60^{\circ} \mathrm{C}$ erwärmt, um Blockcopolymeraggregate in Lösung zu erhalten (Abb. 43a).

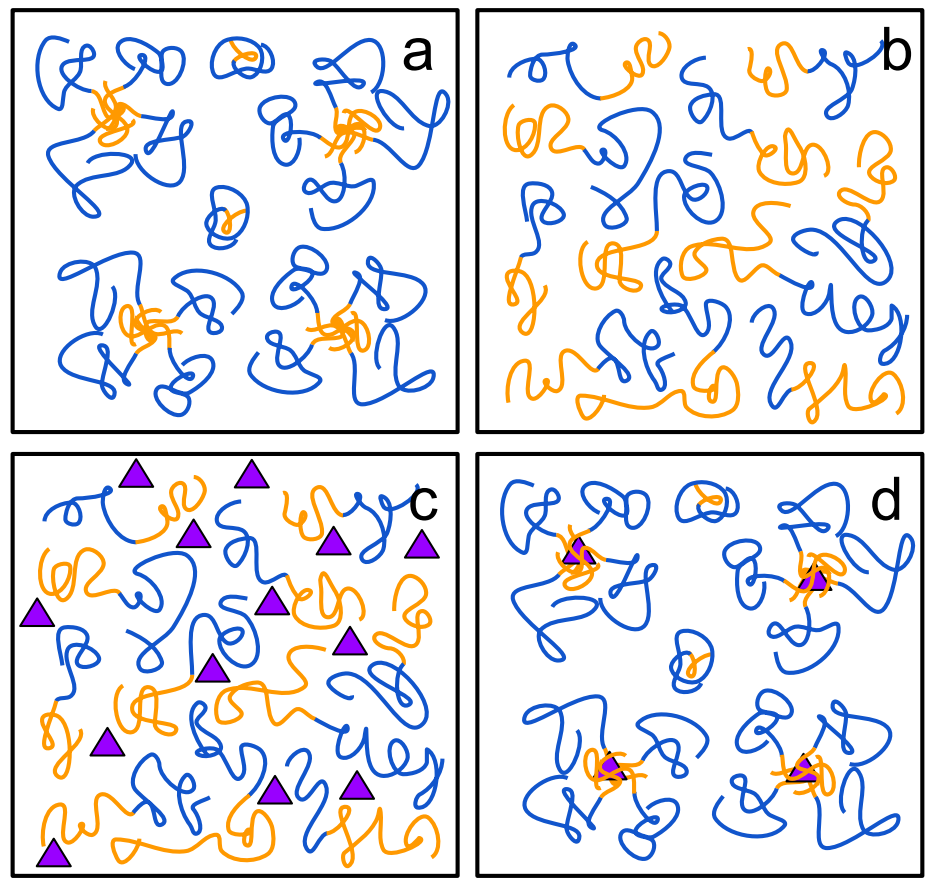

Abbildung 43: Schematische Darstellung der Befüllung von Blockcopolymeraggregaten aus PAzoPMA-b-PDMAEMA (orange-blau) mit Kristallviolett (violett). 
Die Blockcopolymeraggregate wurden durch UV/Vis-Spektroskopie untersucht wie in Abb. 44 gezeigt ist. Es sind zwei Banden bei 350 und $440 \mathrm{~nm}$ zu erkennen, die $\pi \rightarrow \pi^{*}$ und $\mathrm{n} \rightarrow \pi^{*}$-Übergängen zugeordnet werden können. Die Blockcopolymere wurden anschließend erneut mit UV-Licht bestrahlt, um die Aggregate aufzulösen (Abb. 43b) und die Lösung mit Kristallviolett gesättigt (Abb. 43c). Dies ist notwendig, da das Einlagern des Kristallvioletts beim Aggregieren der Blockcopolymere statistisch erfolgt, wenn sich während des Prozesses Kristallviolett zwischen den Blockcopolymerketten befindet. Die Aggregate können durch Zentrifugation und Redispergieren von überschüssigem Kristallviolett getrennt werden. Dies wurde wiederholt, bis die überstehende Lösung keine Banden im UV/Vis-Spektrum zeigte (Abb. 44). So wurden mit Kristallviolett gefüllte Blockcopolymeraggregate erhalten (Abb. 43d).

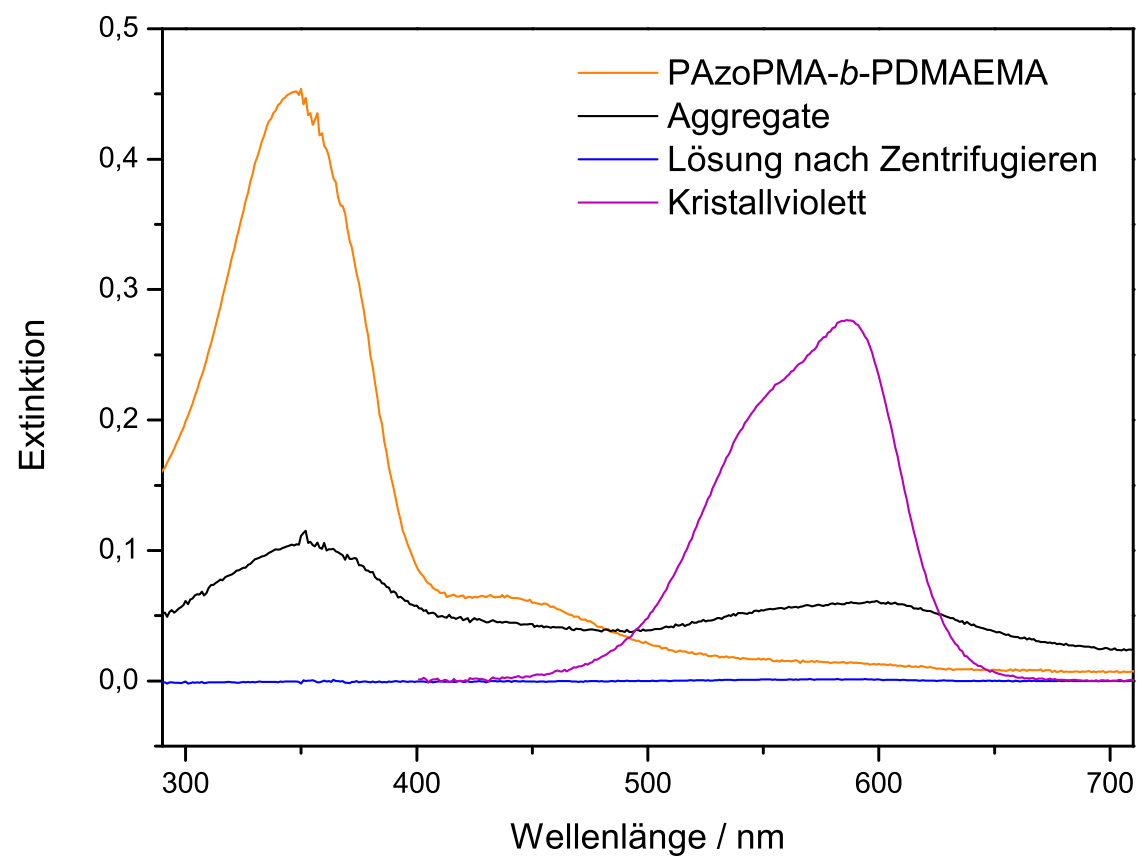

Abbildung 44: UV/Vis-Spektren von PAzoPMA-b-PDMAEMA (orange), der befüllten Polymeraggregate (schwarz), der überstehenden Lösung nach dem Zentrifugieren (blau) und von reinem Kristallviolett (violett). 
Die Aggregate wurden ebenfalls mit UV/Vis-Spektroskopie untersucht (Abb. 44, schwarz). Das Spektrum weist sowohl die Banden des Blockcopolymers als auch die von Kristallviolett auf. Der Einschluss von Kristallviolett in die Polymeraggregate war demnach erfolgreich. Aus den Extinktionen der Komponenten lassen sich Rückschlüsse auf das Verhältnis von Polymer und Farbstoff schließen. Dabei wurden die Extinktionen der Banden des $\pi \rightarrow \pi^{*}$-Überganges bei $350 \mathrm{~nm}$ des Polymers $E_{\mathrm{Azo}}=0,101$ und des Überganges im Kristallviolett bei $600 \mathrm{~nm} E_{\mathrm{KV}}=0,061$ verglichen. Die Extinktionskoeffizienten für diese Übergänge sind durch $\varepsilon_{\mathrm{Azo}}=22000 \mathrm{l} \cdot \mathrm{mol}^{-1} \cdot \mathrm{cm}^{-1}$ und $\varepsilon_{\mathrm{KV}}=86800 \mathrm{l} \cdot \mathrm{mol}^{-1} \cdot \mathrm{cm}^{-1}$ gegeben. ${ }^{59,99}$ Die Schichtdicke beträgt $1 \mathrm{~cm}$. Die Konzentrationen der Azobenzol-Gruppen und von Kristallviolett berechnet sich dann gemäß Gleichung 3 durch

$$
\begin{aligned}
c_{\mathrm{Azo}} & =\frac{E_{\mathrm{Azo}}}{\varepsilon_{\mathrm{Azo}} \cdot d}=\frac{0,101}{22000 \cdot 1} \mathrm{~mol} / \mathrm{l}=4,6 \mu \mathrm{mol} / \mathrm{l} \\
c_{\mathrm{KV}} & =\frac{E_{\mathrm{KV}}}{\varepsilon_{\mathrm{KV}} \cdot d}=\frac{0,061}{86800 \cdot 1} \mathrm{~mol} / \mathrm{l}=0,71 \mu \mathrm{mol} / \mathrm{l} .
\end{aligned}
$$

Da jedes BCP V im Mittel 58 Azobenzol-Gruppen trägt, ergibt sich daraus für die Polymerkonzentration $c_{\mathrm{BCP} V}$ und das Verhältnis aus Kristallviolett- und Polymerkonzentration

$$
\begin{aligned}
c_{\mathrm{BCPV}} & =\frac{c_{\mathrm{Azo}}}{58}=0,079 \mu \mathrm{mol} / 1 \\
\frac{c_{\mathrm{KV}}}{c_{\mathrm{BCPV}}} & =\frac{0,71 \mu \mathrm{mol} / 1}{0,079 \mu \mathrm{mol} / 1}=9,0 .
\end{aligned}
$$

Durch Umrechung mit den molaren Massen der Komponenten kann der Gewichtsanteil an Kristallviolett $w_{\mathrm{KV}}=8,2 \%$ erhalten werden.

\subsection{Fazit und weitere Forschungsperspektiven}

Photoschaltbare Blockcopolymere PAzoPMA- $b$-PBA mit unterschiedlicher PBA-Blocklänge wurden durch RAFT-Polymerisationen hergestellt. Die Molmassenverteilung wurde durch GPC, die Stoffmengenanteile beider Monomerarten via ${ }^{1} \mathrm{H}$-NMR-Spektroskopie ermittelt. 
Durch Spincoating wurden Polymerdünnfilme hergestellt. Das Höhenprofil der Oberflächen dieser Polymere wurde mit dem AFM bestimmt. Der Prozess des thermischen Temperns wurde dabei zeitaufgelöst untersucht. Es konnte gezeigt werden, wie das Blockcopolymer dabei von einem ungeordneten in einen mikrophasenseparierten Zustand übergeht, der einer lamellaren Morphologie zuzuordnen ist. Aus den horizontal zum Substrat angeordnete Lamellen resultierte eine Struktur, die eine Ebene mit einigen Plateaus aufwies. Am Rand der Plateaus wurden senkrecht zum Substrat angeordnete Lamellen beobachtet. Die Phasenzuordnung konnte mittels der durch AFM bestimmten DMT-Moduln und den zugehörigen durch DSC bestimmten Glasübergangstemperaturen erfolgen. Anhand der Oberflächenprofile der Blockcopolymere wurden charakteristische Strukturparameter quantifiziert um einen Vergleich der Strukturen zu ermöglichen. Es zeigte sich, dass die Lamellenbreite und die Breite der PBA-Phase mit der Kettenlänge des PBA-Blockes zunehmen. Ebenso steigt damit die Breite des Bereiches, in dem vertikal angeordnete Lamellen vorliegen.

Ein Blockcopolymer PAzoPMA- $b$-PBA wurde bei konstanten Stoffmengenanteilen mit bis zu 50 Gew.-\% beider zugehöriger Homopolymere gemischt. Dünnfilme der resultierenden BlockcopolymerHomopolymer-Mischungen wurden nach thermischem Tempern mittels AFM untersucht. Alle Komposite wiesen lamellare Morphologien auf, deren Strukturen der des ausgänglichen Blockcopolymers ähnelte. Die Zuordnung der Phasen konnte auch hier durch Bestimmung der mechanischen Eigenschaften durch das AFM erfolgen. Der Einfluss der zugegebenen Menge an Homopolymer auf die Strukturparameter wurde untersucht. Es konnte gezeigt werden, dass alle Strukturparameter mit zunehmender Homopolymerzugabe ansteigen. Der Abstand der horizontal zum Substrat angeordneten Lamellen stieg damit propotional an. Die weiteren Strukturparameter zeigten deutlichere Änderungen, als es sich durch die zugegebene Menge Homopolymer pro- 
gnostizieren ließ, wobei die Defektdichte der Struktur ebenfalls mit der Menge an zugefügtem Homopolymer stark anstieg.

Die Eigenschaften dieser nanostrukturierten Materialien $\mathrm{zu}$ untersuchen bietet interessante Perspektiven für die zukünftige Forschung. Dabei können thermische Eigenschaften wie z. B. die Wärmekapazität oder die Wärmeleitfähigkeit, aber auch mechanische Eigenschaften wie Elastizitätsmodul, Bruchspannung oder Bruchdehnung bestimmt werden. Es ist zudem möglich, dass das Material durch seine anisotrope räumliche Struktur auch anisotrope Eigenschaften aufweist. Diese könnten zudem vom Schaltzustand des integrierten Photoschalters abhängig sein.

PAzoPMA- $b$-PDMAEMA kann in Propylencarbonat lichtinduziertspaltbare Blockcopolymeraggregate bilden. Solche Aggregate konnten erfolgreich mit dem Farbstoff Kristallviolett befüllt und durch UV/VisSpektroskopie charakterisiert werden. Aus den Extinktionen konnte ein Massenanteil von 8,2 \% Kristallviolett bestimmt werden.

Auf diesen Ergebnissen aufbauend kann das System weiter verbessert werden. Im Hinblick auf die Verwendung von Polymeraggregaten zur Wirkstoffgabe muss das System muss biokompatibel sein, um eine Verabreichung zu ermöglichen. Dafür sind wesentliche Punkte zu beachten: Da lebendes Gewebe wasserbasiert ist, ist es essentiell, dass das Blockcopolymer wasserlöslich ist. Ebenso muss eine Möglichkeit bestehen, das Polymer nach abgeschlossener Wirkstoffabgabe abzubauen. Dazu müssen funktionelle Gruppen in das Polymerrückgrat eingebaut werden, die einen Abbau in kleinere, ausscheidbare Moleküle ermöglichen. Zudem sollte der Massenanteil an eingeschlossenen Molekülen genau einstellbar sein, sodass die Dosierung an den Anwendungsbereich angepasst werden kann. Eine geringere Beladung der Aggregate sollte durch eine verringerte Zugabe des einzuschließenden Stoffes einfach möglich sein. Um eine höhere Beladung zu ermöglichen, werden neue Strategien benötigt. Eine Möglichkeit dafür könnte der Einbau schwacher attraktiver Wechselwirkungen, wie beispielweise Wasserstoffbrücken oder Dipol-Dipol-Wechselwirkungen, zwischen Poly- 
mer und dem einzuschließenden Stoff sein. Dadurch könnte sich die Konzentration des einzuschließenden Stoffes in der räumlichen Nähe des Polymers erhöhen und so eine höhere Beladung resultieren. 


\section{Untersuchung der Wärmeleitfähigkeit makromolekularer Systeme}

Eines der zentralen Forschungsgebiete der Polymerchemie ist die Entwicklung von maßgeschneiderten Hochleistungsmaterialien. Neben ihren Vorteilen wie ihrer Flexibilität, der geringen Dichte oder guten chemischen Stabilität stellt insbesondere die geringe Wärmeleitfähigkeit ein Problem dar. Bei elektronischen Anwendungen kann die Abwärme nur schlecht durch das Polymer abtransportiert werden, wodurch die Belastung des Materials und damit auch der thermische Zerfall hoch ist. ${ }^{100,101}$ Die Verbesserung der Wärmeleitfähigkeit ist daher eine zentrale Herausforderung, die bei der Entwicklung neuer Materialien überwunden werden muss.

\subsection{Hintergründe}

Die Wärmeleitfähigkeit ist eine wichtige Größe, um das thermische Verhalten von Materialien zu beschreiben. Sie ist dabei nicht nur für hochfunktionalisierte Materialien wie z. B. Komposite aus Polymer und Kohlenstoffnanoröhrchen, sondern auch für klassische Werkstoffe wie Stahl oder Beton wichtig. ${ }^{102-104}$ Die Wärmeleitfähigkeit $\kappa$ beschreibt wie thermische Energie in einem System mit einem Temperaturgradienten $\nabla T$ transportiert wird. Sie hängt mit dem Wärmefluss $Q$ durch das Fouriersche Gesetz

$$
Q=-\kappa \cdot \nabla T
$$

zusammen. Dieses kann für den eindimensionalen Wärmetransport in $x$-Richtung als

$$
Q=-\kappa \cdot \frac{\mathrm{d} T}{\mathrm{~d} x}
$$

geschrieben werden. Wärmetransport kann im Wesentlichen durch Strahlung, Konvektion oder Konduktion erfolgen. ${ }^{105,106}$ In Festkörpern 
ist dabei die Konduktion hauptsächlich für die Wärmetransporteigenschaften verantwortlich. Die Energie wird durch die Anregung der Vibration benachbarter Teilchen, meistens durch Stöße, weitergegeben, wie in Schema 45 gezeigt ist. Dabei findet kein Transport von Materie statt. Die Wärmeleitfähigkeiten einiger typischer Materialien bei Raumtemperatur sind in Tabelle 11 zusammengefasst. Erkennbar ist, dass Polymere nur geringe Wärmeleitfähigkeiten aufweisen. Mit zunehmendem Anteil kristalliner Bereiche steigt ihre Wärmeleitfähigkeit an. Metalle hingegen zeichnen sich durch Wärmeleitfähigkeiten aus, die etwa 1000-fach größer sind, als die der Polymere. Sehr hohe Wärmeleitfähigkeiten treten bei Kohlenstoff-basierten Materialien wie Diamant, Graphen oder Kohlenstoffnanoröhrchen auf. Die verschiedenen Wärmeleitfähigkeiten lassen sich durch die unterschiedlichen Transportmechanismen, resultierend aus der Struktur dieser Materialien, erklären.

Tabelle 11: Wärmeleitfähigkeiten $\kappa$ verschiedener ausgewählter Materialien bei Raumtemperatur. ${ }^{107}$

\begin{tabular}{lcc}
\hline Material & $\kappa / \mathrm{W} /(\mathrm{m} \mathrm{K})$ & Quelle \\
\hline Polystyrol (PS) & $0,10-0,15$ & {$[107]$} \\
Polypropylen (PP) & $0,11-0,17$ & {$[107]$} \\
Polymethylmethacrylat (PMMA) & $0,16-0,25$ & {$[107]$} \\
Polydimethylsiloxan (PDMS) & 0,25 & {$[108]$} \\
Polytetrafluorethylen (PTFE) & 0,27 & {$[107]$} \\
Polyethylen (LDPE) & $0,30-0,34$ & {$[107]$} \\
Polyethylen (HDPE) & $0,33-0,53$ & {$[107]$} \\
Polyethylen (gestreckte Kette) & 104 & {$[109]$} \\
Aluminium & 234 & {$[110]$} \\
Gold & 314 & {$[111]$} \\
Kupfer & 386 & {$[112]$} \\
Silber & 427 & {$[113]$} \\
Diamant & 1000 & {$[114]$} \\
Kohlenstoffnanoröhrchen & $1000-4000$ & {$[115]$} \\
Graphen & 3000 & {$[116]$} \\
\hline
\end{tabular}




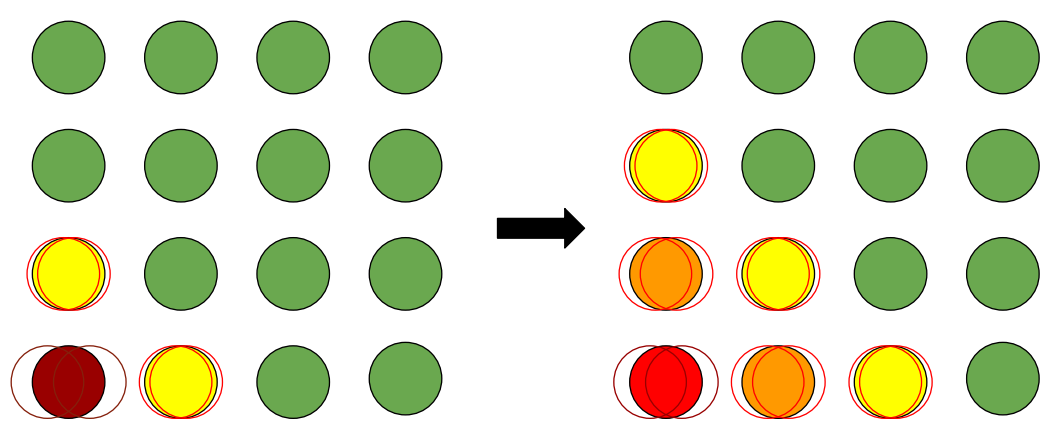

Abbildung 45: Schematische Darstellung des Wärmetransportes durch Vibration in einem Material. ${ }^{105}$

In amorphen Polymeren findet der Wärmetransport hauptsächlich über Phononen statt, da die Elektronen im System nicht frei beweglich sind. ${ }^{108}$ Die Wärme wird dabei jedoch nicht gerichtet weitergeleitet, sondern verursacht ungeordnete Rotationen und Vibrationen der Atome der Kette um ihre Gleichgewichtsposition herum. Alternativ wird sie an benachbarte Ketten weitergegeben. Die Wärmeleitfähigkeit durch Phononen kann durch die Debye-Gleichung

$$
\kappa=\frac{c_{p} \cdot \nu \cdot l}{3}
$$

beschrieben werden. Dabei ist $c_{p}$ die Wärmekapazität bei konstantem Druck $p, \nu$ die Phononenfrequenz und $l$ die mittlere freie Weglänge der Phononen. Da die mittlere freie Weglänge der Phononen in amorphen Polymeren oft nur wenige Angström beträgt, ist folglich auch die Wärmeleitfähigkeit gering. ${ }^{117}$

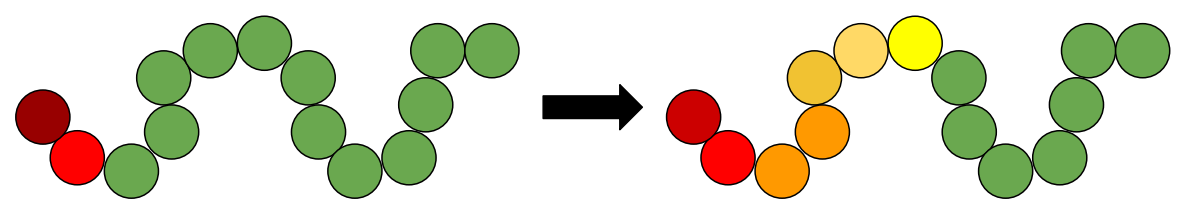

Abbildung 46: Schematische Darstellung des Wärmetransportes durch Vibration in einem Polymer. Warme Teilchen sind dabei in rot, kalte Teilchen in grün dargestellt. ${ }^{105}$ 

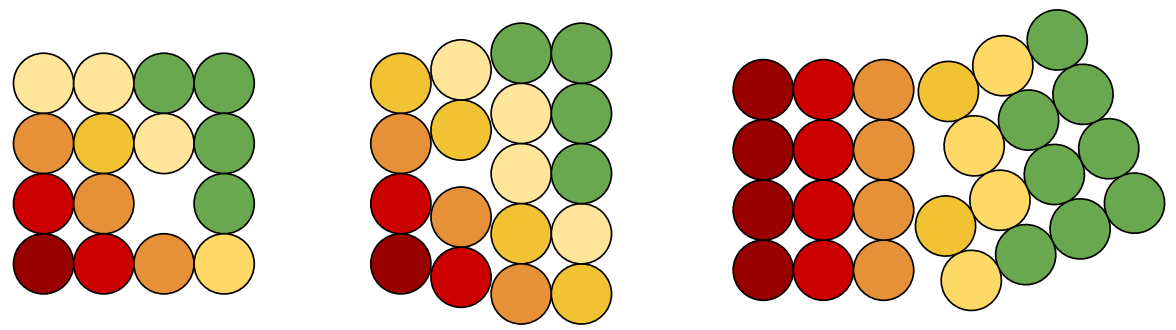

Abbildung 47: Schematische Darstellung des Wärmetransportes in Systemen mit verschiedenen Defekten: Punktdefekt (Links), Versetzung (Mitte) und Korngrenze (Rechts). Warme Teilchen sind dabei in rot, kalte Teilchen in grün dargestellt. ${ }^{105}$

Die ungeordnete Struktur eines amorphen Polymers kann für ihr thermisches Verhalten mit einer Kombination vieler Defekte verglichen werden. Drei Beispiele für Defekte in einem System, Punktdefekt, Versetzung und Korngrenze, sind in Abbildung 47 dargestellt. Defekte fungieren als Streustellen für Phononen, sodass sich bei vielen Defekten die mittlere freie Weglänge der Phononen verringert und somit auch die Wärmeleitfähigkeit sinkt. Durch eine Verlängerung der mittleren freien Phononenweglänge kann somit auch die Wärmeleitfähigkeit der Polymere erhöht werden. Auch die Bindungsenergie und Steifigkeit eines Materials hat einen Einfluss auf die Wärmeleitfähigkeit. In einem Diamanten ist beispielsweise ein Kohlenstoffatom an vier weitere Kohlenstoffatome gebunden, die wiederum an weitere Kohlenstoffatome gebunden sind. Daraus folgt eine sehr starre Struktur. In einem Polymer hingegen ist eine Bewegung der Kette möglich, sodass die Freiheit der Vibration hier weniger stark eingeschränkt ist. Daraus resultiert, dass die Weitergabe von Wärme durch Vibrationen langsamer und somit die Wärmeleitfähigkeit geringer ist. ${ }^{105}$ Dieser Effekt wird beim Vergleich von amorphen und kristallinen Polymeren deutlich. Beispielsweise konnte für PTFE und PE gezeigt werden, dass die Wärmeleitfähigkeit mit dem Grad der Kristallinität zunimmt. ${ }^{118,119}$ Die Wärmeleitfähigkeit nimmt jedoch nicht so stark zu, dass Werte in der Größenordnung von Metallen erreicht werden. Wird die Struktur von Polymeren so modifiziert, dass einige wenige Ketten nebeneinander 
stark gestreckt vorliegen, so kann die Wärmeleitfähigkeit weiter erhöht werden. Für Nanofasern aus PE mit einem Durchmesser von 50 bis $500 \mathrm{~nm}$ konnte so eine Wärmeleitfähigkeit von $104 \mathrm{~W} /(\mathrm{K} \cdot \mathrm{m})$ erhalten werden. ${ }^{109}$ Simulationen sagen voraus, dass für eine einzelne Kette aus PE eine maximale Wärmeleitfähigkeit von $350 \mathrm{~W} /(\mathrm{K} \cdot \mathrm{m})$ erreicht werden kann. ${ }^{120}$

Die Wärmeleitfähigkeit von Metallen liegt im Bereich einiger hundert $\mathrm{W} /(\mathrm{K} \cdot \mathrm{m})$. Neben der längeren mittleren freien Weglänge der Phononen im Metall leisten die frei beweglichen Elektronen einen Beitrag zur Wärmeleitfähigkeit, die sich nach

$$
\kappa=\kappa_{\mathrm{e}}+\kappa_{\mathrm{p}}
$$

aus der Wärmeleitfähigkeit durch Phononen $\kappa_{\mathrm{p}}$ und dem Beitrag der Elektronen $\kappa_{\mathrm{e}}$ zusammensetzt. Die Wärmeleitfähigkeit durch Elektronen ist durch das Wiedemann-Franz'sche Gesetz

$$
\kappa_{\mathrm{e}}=L_{0} \sigma_{\mathrm{e}} T
$$

mit der Lorentz-Konstanten $L_{0}$ und der elektrischen Leitfähigkeit $\sigma_{\mathrm{e}}$ gegeben. ${ }^{105}$ Für Metalle dominiert der elektronische Anteil, sodass für die Wärmeleitfähigkeit

$$
\kappa \approx \kappa_{\mathrm{e}}
$$

gilt. ${ }^{121}$ Kohlenstoff-basierte Materialien wie Diamant, Graphen oder Kohlenstoffnanoröhrchen weisen Wärmeleitfähigkeiten von mehr als 1000 W/(K·m) auf. Diamant besteht aus sp ${ }^{3}$-hybridisierten Kohlenstoffatomen, welche tetraedrisch von weiteren Kohlenstoffatomen umgeben sind, wie Abbildung 48 zeigt. Graphen und Kohlenstoffnanoröhrchen weisen die selbe Grundstruktur aus sp²-hybridisiertem Kohlenstoff auf, wie in Abbildung 49 zu sehen ist. Graphen besteht dabei aus zweidimensionalen Ebenen der Struktur, während bei Kohlenstoffnanoröhrchen die Struktur zu einer Röhre geformt ist. Alle Strukturen 


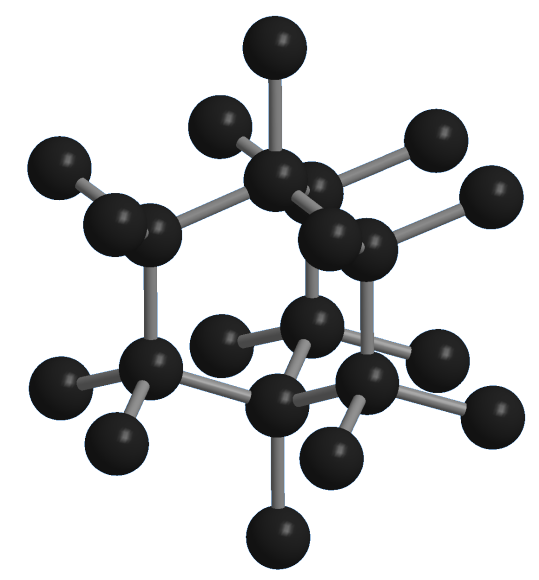

Abbildung 48: Ausschnitt aus der Struktur von Diamant.

sind dabei extrem starr, was zu einer sehr großen mittleren freien Weglänge der Phononen im Material führt. ${ }^{122}$ Defekte der Struktur führen auch bei diesen Materialien zu einer Verringerung der Wärmeleitfähigkeit, da sie die mittlere freie Weglänge der Phononen verkürzen. ${ }^{123}$ Der elektronische Anteil der Wärmeleitfähigkeit ist in Kohlenstoff-basierten Systemen vernachlässigbar. Diamant ist nicht elektrisch leitfähig, Graphen und Kohlenstoffnanoröhrchen haben eine elektrische Leitfähigkeit, die aber nach Gleichung 26 nur etwa 0,01 W/(K·m) zur gesamten Wärmeleitfähigkeit beiträgt. ${ }^{105}$ Bei Kohlenstoffnanoröhrchen ist die Wärmeleitfähigkeit zudem vom Durchmesser der Röhrchen abhängig. Je kleiner der Durchmesser, desto höher die Wärmeleitfähigkeit. ${ }^{124}$

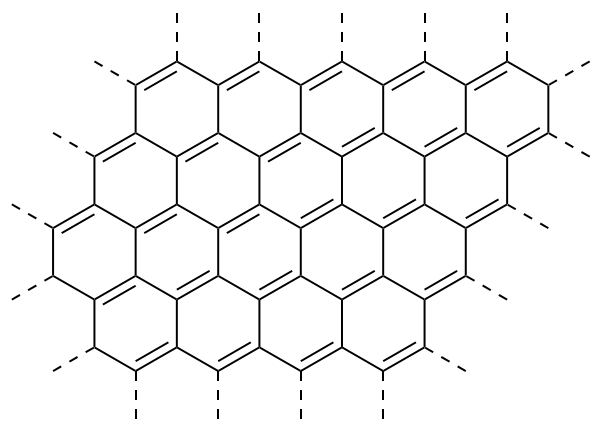

Abbildung 49: Ausschnitt aus der Struktur von Graphen und Kohlenstoffnanoröhrchen. 


\subsubsection{Einflüsse auf die Wärmeleitfähigkeit von Polymeren}

Die Wärmeleitfähigkeit eines Polymers hängt im Wesentlichen von zwei Faktoren ab: Der molekularen Struktur des Polymers und der Orientierung der Ketten im Material. ${ }^{125}$ Für die molekulare Struktur soll hier auf vier Faktoren eingegangen werden. Das Polymerrückgrat (i), Seitengruppen (ii), Wasserstoffbrücken (iii) und Vernetzungen (iv).

(i) Der Wärmetransport entlang des Polymerrückgrats wird durch die Streckbarkeit der Bindungen, der Knickbarkeit sowie der Fähigkeit um eine Bindung zu rotieren bestimmt. Ein starres Polymerrückgrat führt zu gestreckteren Ketten und damit zu einer höheren Wärmeleitfähigkeit. ${ }^{126,127} \pi$-Bindungen im Polymerrückgrat erhöhen die Kettensteifigkeit weiter, insbesondere wenn sie, wie z. B. in Aromaten, in Konjugation vorliegen. ${ }^{128-130}$

(ii) Die Wärmeleitfähigkeit eines Polymers nimmt ab, wenn nichtstrukturierende Seitengruppen eingeführt werden. Dabei fällt sie mit der Anzahl und der Größe der Seitengruppen. ${ }^{131}$ Dies kann auf eine verringerte Kristallinität des Polymers sowie auf erhöhte Phononenstreuung zurückgeführt werden. ${ }^{131,132}$ Seitenketten können jedoch auch zu einer höheren strukturellen Ordnung im System führen und so die Wärmeleitfähigkeit erhöhen. ${ }^{133,134}$

(iii) Wasserstoffbrücken können die Wärmeleitfähigkeit eines Polymers durch die Ausbildung von Verbindungen zwischen den Ketten verbessern. ${ }^{135,136}$ Bei Polymermischungen muss eine gleichmäßige Mischung der Polymere gewährleistet sein, damit sich Wasserstoffbrücken und damit kontinuierliche Mikrostrukturen aufbauen können. ${ }^{137,138}$ Wasserstoffbrücken verringern zudem durch die weiteren Verknüpfungspunkte die Beweglichkeit der Ketten, was mit einer weiteren Erhöhung der Wärmeleitfähigkeit durch verringerte Phononenstreuung einhergeht. ${ }^{139}$ Sie erhöhen die Wärmeleitfähigkeit jedoch nicht zwangsläufig, sondern können auch die Bindungen im Polymerrück- 
Wärmeleitfähigkeit makromolekularer Systeme

grat schwächen, ${ }^{140}$ oder zu verstärkter Phononenstreuung führen. ${ }^{141}$

(iv) Die Wärmeleitfähigkeit eines Polymers kann zudem mit der Anzahl der Vernetzungen zwischen den Ketten zunehmen. ${ }^{142,143}$ Molekulardynamik-Simulationen zeigten, dass die kovalenten Bindungen nur etwa $20 \%$ zur gesamten Wärmeleitfähigkeit beitragen. Der restliche Beitrag stammt von nichtbindenden Interaktionen, deren Anzahl durch die Vernetzung deutlich ansteigt, da die Ketten durch die Vernetzungen in räumlicher Nähe gehalten werden. ${ }^{144}$ Die Wärmeleitfähigkeit kann durch Vernetzung jedoch auch sinken, da das Ausbilden gut leitender kristalliner Bereiche durch die Vernetzungen gehindert ist. $^{145,146}$

Die Wärmeleitfähigkeit eines Polymers wird neben der molekularen Struktur auch durch die Ausrichtung der Ketten bestimmt. Für hochkristallines, gestrecktes PE, bei dem die Ketten entlang der Streckachse ausgerichtet sind, wurde eine, für Polymere sehr große Wärmeleitfähigkeit von $104 \mathrm{~W} /(\mathrm{K} \cdot \mathrm{m})$ gemessen. ${ }^{109}$ Eine solche Ausrichtung kann je nach Polymer unter anderem durch themisches Tempern ${ }^{147,148}$ oder mechanisches Strecken erreicht werden. ${ }^{109,149,150}$ Durch die spezielle Anordnung ist die Kristallinität in solch einem Polymer sehr hoch, wodurch die mittlere freie Weglänge der Phononen im Material größer und damit auch die Wärmeleitfähigkeit verbessert ist.

Um die Eigenschaften von Polymeren zu modifizieren werden oft Füllstoffe beigemischt. Dabei werden die Vorteile beider Materialien kombiniert. Das Polymer bleibt leicht und gut zu verarbeiten, die gewünschte Eigenschaft, wie z. B. die Wärmeleitfähigkeit oder der Elastizitätsmodul, wird jedoch gezielt verbessert. ${ }^{151}$ Die Vielfalt an genutzten Füllstoffen reicht von Keramiken wie Bornitrid, ${ }^{152-155}$ Magnesiumoxid, ${ }^{156-158}$ Aluminiumoxid, ${ }^{159-161}$ Siliciumcarbid ${ }^{162-164}$ oder Zirkoniumdiborid $^{165,166}$ über Metalle wie Kupfer, ${ }^{167-169}$ Silber, ${ }^{170-172}$ Aluminium, ${ }^{173,174}$ Eisen $^{175}$ und Gold ${ }^{176,177}$ zu Kohlenstoff-basierten Füll- 
stoffen wie Kohlenstoffnanoröhrchen, ${ }^{178-180}$ Graphen ${ }^{181-183}$ oder Diamant. ${ }^{184-186}$ Die Grenzfläche zwischen Polymer und Füllstoff stellt in solchen Systemen jedoch eine Barriere im Wärmetransport dar, da sie zu Phononenstreuung führen kann. ${ }^{105,187}$ 


\subsection{Untersuchung der Wärmeleitfähigkeit photoschaltbarer Polymere}

Um den Einfluss einer photoschaltbaren Gruppe auf die Wärmeleitfähigkeit eines Polymers zu untersuchen, wurde PAzoPMA durch RAFT-Polymerisation von AzoPMA hergestellt, wie in Kapitel 6.11 beschrieben ist. Das Polymer wies eine mittlere Molmasse von $M_{\mathrm{n}}=1,15 \cdot 10^{4} \mathrm{~g} \cdot \mathrm{mol}^{-1}$ und eine Dispersität von $Ð=1,40$ auf. Zur Bestimmung der Wärmeleitfähigkeit mittels transienter Thermoreflektrometrie (TTR) wurde das Polymer durch Spincoaten einer Lösung in PGMEA auf ein Substrat aufgebracht. Die Schichtdicke des Polymerfilms wurde durch Ellipsometrie bestimmt und die Probe mit $50 \mathrm{~nm}$ Kupfer bedampft. Die Kupferoberfläche wurde während der Messung durch einen gepulsten Laser aufgeheizt und die Oberflächentemperatur zeitaufgelöst gemessen. Zunächst wurde durch eine Messreihe von fünf Proben mit unterschiedlichen Schichtdicken des Polymers (48,6, 92,0, 184,5, 278,1 und 308,0 nm) auf Si verifiziert, dass sich die Wärmeleitfähigkeit von PAzoPMA nicht mit der Schichtdicke ändert.

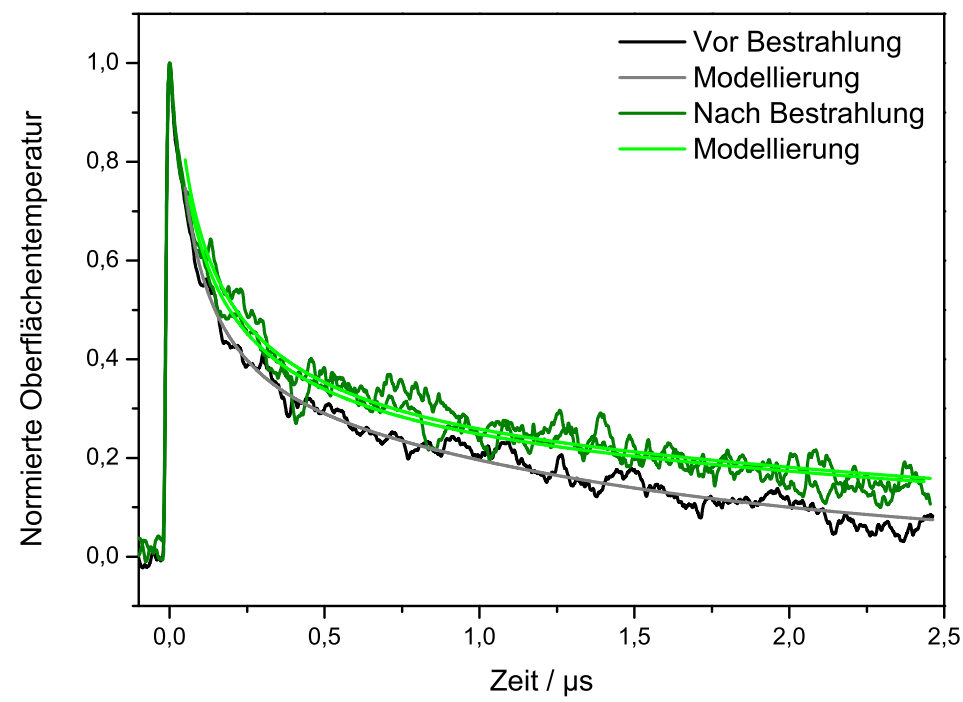

Abbildung 50: Auftragung der normierten Oberflächentemperatur des Kupferfilms gegen die Zeit nach dem Heizpuls für trans-PAzoPMA und cis-PAzoPMA. 
Da Kupfer undurchlässig für UV-Licht ist, muss, um das Polymer nach dem Auftragen des Kupferfilmes bestrahlen zu können, ein für UVLicht transparentes Substrat verwendet werden. Für die Messungen zur Wärmeleitfähigkeit in Abhängigkeit des Schaltzustandes wurde deshalb Magnesiumoxid als Substrat eingesetzt. Der für die Messung verwendete Dünnfilm des Polymers wies eine Schichtdicke von 769,4 nm auf. Für den trans-Zustand (Abbildung 50, schwarz) wurde eine Wärmeleitfähigkeit von $0,385 \mathrm{~W} /(\mathrm{K} \cdot \mathrm{m})$ bestimmt. Im Vergleich zu klassischen Polymeren, deren Wärmeleitfähigkeit in der Regel zwischen 0,1 und $0,5 \mathrm{~W} /(\mathrm{K} \cdot \mathrm{m})$ liegt, ${ }^{107}$ ist dies ein relativ hoher Wert. Ein Grund für die relativ hohe Wärmeleitfähigkeit von trans-PAzoPMA könnte die Ausbildung von $\pi-\pi$-Wechselwirkungen zwischen den AzobenzolGruppen unterschiedlicher Ketten sein, wie Abbildung 51 zeigt. Analog zur Ausbildung von Wasserstoffbrücken könnten diese Wechselwirkungen die Wärmeübertragung zwischen den verschiedenen Ketten verbessern. Zudem wären solche Bereiche in ihrer Struktur ähnlich zu einem kristallinen Bereich im Polymer.
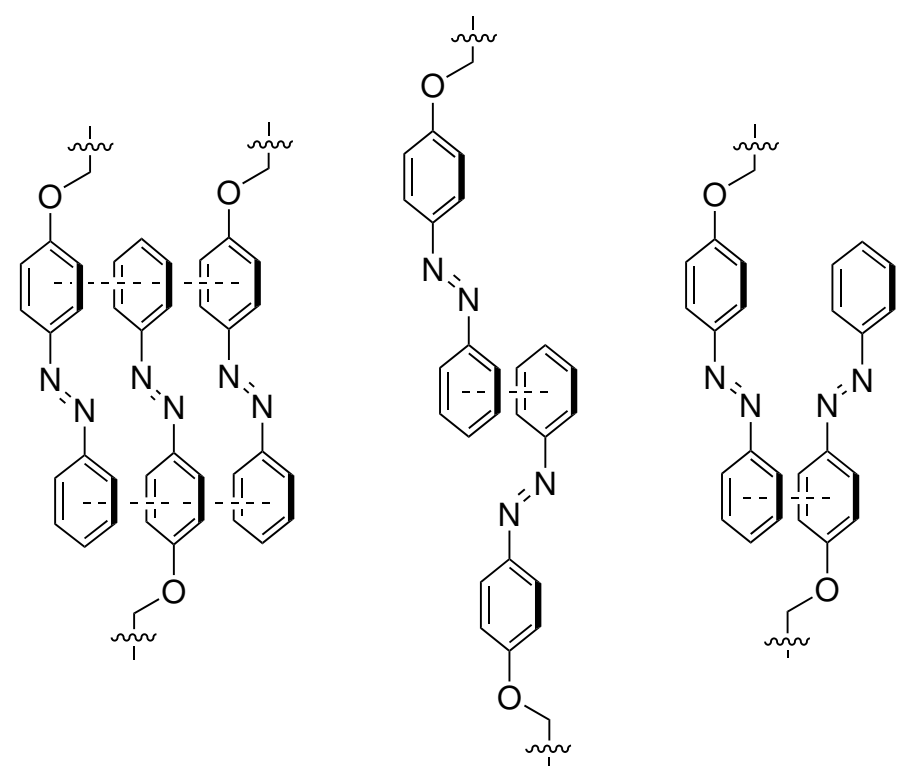
Abbildung 51: Beispiele möglicher $\pi-\pi$-Wechselwirkungen zwischen Azoben- zolgruppen in PAzoPMA.


Wärmeleitfähigkeit makromolekularer Systeme

Nach Bestrahlung mit UV-Licht und der damit einhergehenden Isomerisierung in die cis-Konfiguration sinkt die Wärmeleitfähigkeit von PAzoPMA um etwa $60 \%$ auf $0,160 \mathrm{~W} /(\mathrm{K} \cdot \mathrm{m})$. Durch die Isomerisierung ändert sich die Geometrie der Azobenzol-Gruppe. In der transKonfiguration stehen die aromatischen Ringe im Winkel von $180^{\circ} \mathrm{zu}-$ einander und sind in einer Ebene angeordnet. Durch die Isomerisierung ändert sich dieser Winkel auf $60^{\circ}$ und die Ringe sind aus der Ebene der N-N-Bindung gedreht. Mögliche $\pi-\pi$-Wechselwirkungen könnten durch diese Geometrieänderung vermindert werden und der Wärmetransport zwischen unterschiedlichen Ketten wird folglich erschwert.

\subsection{Wärmeleitfähigkeit von PMMA in Abhängigkeit von der Kettenlänge}

\begin{tabular}{lcccccr}
\hline In & diesem & Kapitel & \multicolumn{2}{c}{ werden } & Ergebnisse vorgestellt, die & gemeinsam \\
mit & Lena Dyckhoff & im & Rahmen ihrer Bachelorarbeit "Untersuchung \\
der & Wärmeleitfähigkeit & von & Polymer-Nanokompositen“ erhalten wurden.
\end{tabular}

Durch Simulationen von Pigard et al. konnte gezeigt werden, dass die Wärmeleitfähigkeit von Polymeren von der Kettenlänge abhängig ist. ${ }^{188}$ Wie in Abbildung 52 zu sehen ist, wird vorhergesagt, dass die Wärmeleitfähigkeit für kurze Ketten mit der Molmasse steigt und für lange Ketten gegen einen konstanten Wert strebt. Wann diese Bereiche ineinander übergehen, wird durch den Simulationsparameter $\lambda_{\mathrm{x}}$ beschrieben. Je kleiner $\lambda_{x}$, desto früher strebt die Wärmeleitfähigkeit einen konstanten Wert an. Um den Einfluss der Kettenlänge auf die Wärmeleitfähigkeit eines Polymers experimentell zu untersuchen, wurde eine Serie von PMMA durch RAFT-Polymerisation hergestellt, wie in Kapitel 6.11 gezeigt ist. Die mittlere Molmasse der Polymere wurde durch GPC bestimmt. Aus den ermittelten Glasübergangstemperaturen wird ersichtlich, dass bei einer Bestimmung der Wärmeleitfähigkeit bei Raumtemperatur der Glasübergang bei sämtlichen Proben nicht erreicht werden sollte. Die Wärmeleitfähigkeit wurde aus den Messungen der TTR bestimmt. 
Wärmeleitfähigkeit makromolekularer Systeme

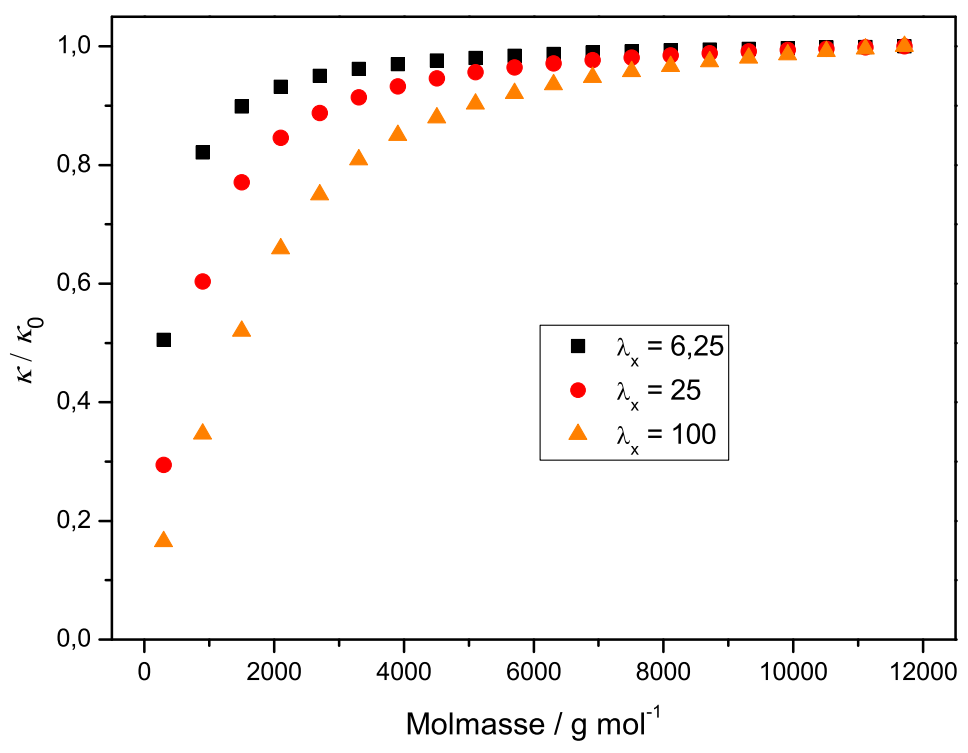

Abbildung 52: Simulierte Wärmeleitfähigkeit von PMMA in Abhängigkeit von der Molmasse für verschiedene Werte des Parameters $\lambda_{\mathrm{x}}$. Die Simulation wurde von Louis Pigard am Institut für Theoretische Physik der Universität Göttingen durchgeführt. ${ }^{188}$

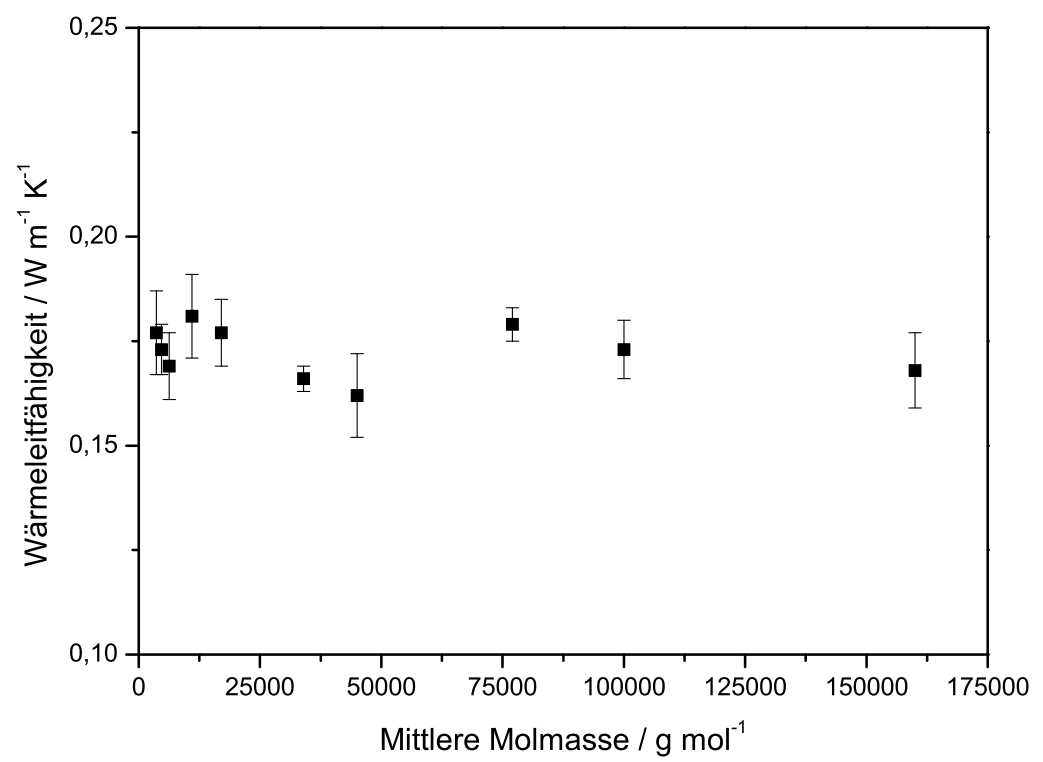

Abbildung 53: Auftragung der Wärmeleitfähigkeit der PMMA-Dünnfilme gegen die mittlere Molmasse des Polymers. 
Die bestimmten Größen sind in Tabelle 12 zusammengefasst. Die Glasübergangstemperaturen zeigen den erwarteten Trend nach Flory und Fox (siehe Kapitel 7.5). ${ }^{189}$ Die ermittelten Wärmeleitfähigkeiten sind in Abbildung 53 gezeigt. Es ist deutlich zu erkennen, dass keine Abhängigkeit der Wärmeleitfähigkeit von der Molmasse des Polymers besteht. Die Wärmeleitfähigkeit der Proben ist zudem im Rahmen des Fehlers nahezu konstant, sodass ein durchschnittlicher Wert von $\bar{\kappa}=(0,173 \pm 0,006) \mathrm{W} /(\mathrm{K} \cdot \mathrm{m})$ bestimmt wurde. Alle erhaltenen Werte liegen in einem Intervall von $5 \%$ um diesen Mittelwert.

Der Wärmetransport in Polymeren ist entlang einer Kette wesentlich schneller, als zwischen unterschiedlichen Ketten. ${ }^{109}$ Ausgehend von reinem Monomer, bei dem nur intermolekularer Wärmetransport relevant ist, steigt die Wärmeleitfähigkeit für Dimere bis hin zu Oligomeren an, da der intramolekulare Wärmetransport schneller ist. Die Wärmeleitfähigkeit kann jedoch nicht endlos mit der Kettenlänge ansteigen. Für lange Ketten ist die schlechte intermolekulare Wärmeleitfähigkeit maßgeblich dafür, dass eine Verlängerung einer Kette nahezu keinen Effekt mehr auf die Wärmeleitfähigkeit des Systems zeigt.

Tabelle 12: Übersicht über die mittlere Molmasse $M_{\mathrm{n}}$, die Dispersität $\oslash$, die Glasübergangstemperatur $T_{\mathrm{g}}$ und die Wärmeleitfähigkeit $\kappa$ von PMMA I-X.

\begin{tabular}{lcccc}
\hline & $M_{\mathrm{n}} / 10^{3} \mathrm{~g} \cdot \mathrm{mol}^{-1}$ & $Đ$ & $T_{\mathrm{g}} /{ }^{\circ} \mathrm{C}$ & $\kappa / \mathrm{W} /(\mathrm{m} \mathrm{K})$ \\
\hline PMMA I & 3,6 & 1,09 & 59,1 & $0,177 \pm 0,010$ \\
PMMA II & 4,7 & 1,11 & 65,8 & $0,173 \pm 0,006$ \\
PMMA III & 6,3 & 1,08 & 75,5 & $0,169 \pm 0,008$ \\
PMMA IV & 11,0 & 1,08 & 86,0 & $0,182 \pm 0,009$ \\
PMMA V & 17,0 & 1,07 & 91,0 & $0,177 \pm 0,008$ \\
PMMA VI & 34,0 & 1,09 & 99,5 & $0,166 \pm 0,003$ \\
PMMA VII & 45,0 & 1,11 & 100,9 & $0,162 \pm 0,011$ \\
PMMA VIII & 77,0 & 1,25 & 98,7 & $0,179 \pm 0,004$ \\
PMMA IX & 100 & 1,36 & 110,1 & $0,173 \pm 0,007$ \\
PMMA X & 160 & 2,03 & 115,5 & $0,168 \pm 0,009$ \\
\hline
\end{tabular}




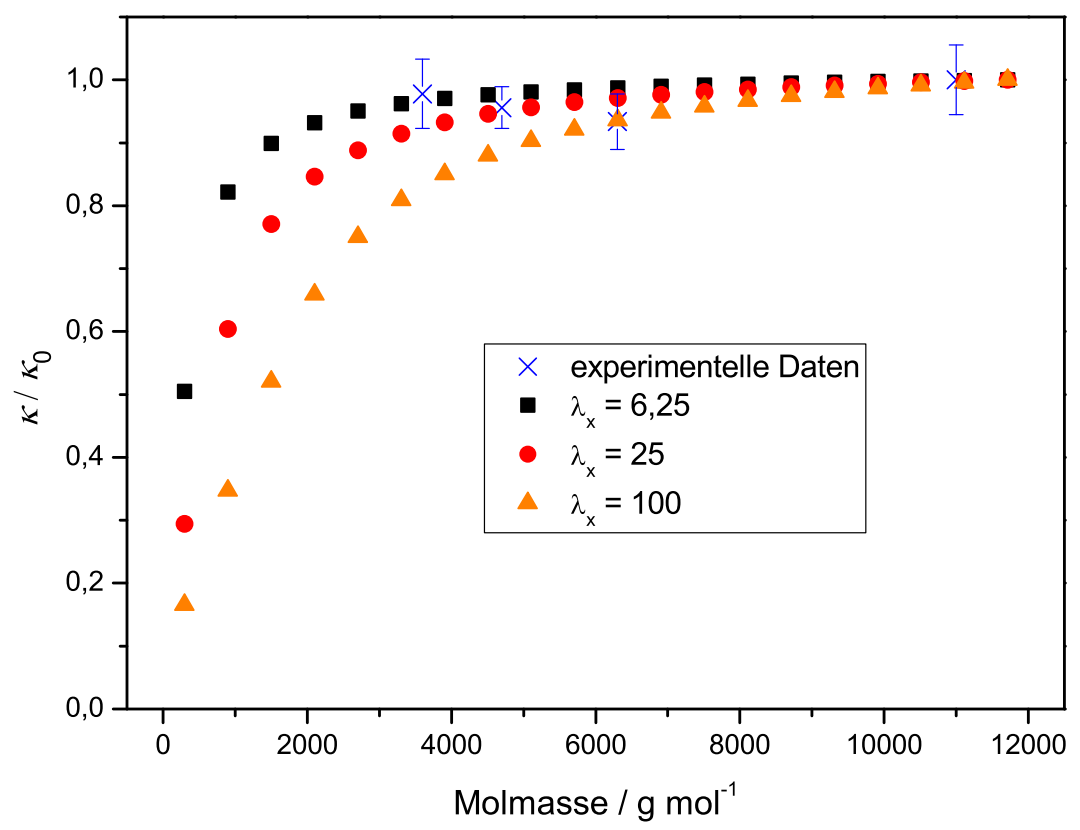

Abbildung 54: Vergleich der Simulation mit den experimentellen Daten der Wärmeleitfähigkeit von PMMA mit unterschiedlicher Molmasse.

Werden die experimentellen Befunde mit der Simulation verglichen, wie in Abbildung $54 \mathrm{zu}$ sehen ist, zeigt sich, dass diese mit den theoretischen Vorhersagen übereinstimmen. Die aus dem Experiment gewonnenen Informationen können genutzt werden, um die Simulation $\mathrm{zu}$ verbessern. Der Parameter $\kappa_{0}$ kann durch $\bar{\kappa}$ beschrieben werden. Des Weiteren kann eine Abschätzung für $\lambda_{\mathrm{x}}$ getroffen werden. Es zeigt sich, dass $\lambda_{\mathrm{x}}<25$ sein sollte. Für eine genauere Beschreibung würden weitere Werte für Polymere mit unter $3600 \mathrm{~g} \cdot \mathrm{mol}^{-1}$ benötigt werden. PMMA mit einer so geringen molaren Masse haben jedoch aufgrund der aufwändigen Synthese und der zu erwartenden geringen Glasübergangstemperatur für technische Anwendungen keine Relevanz. Eine Bestimmung der Wärmeleitfähigkeit solcher Proben oberhalb der Glasübergangstemperatur ist nicht sinnvoll, da sich die Wärmeleitfähigkeit mit dem Phasenübergang ändern kann und ein Vergleich der Ergebnisse somit nicht möglich ist. 
Wärmeleitfähigkeit makromolekularer Systeme

\subsection{Wärmeleitfähigkeit von Polymer-Gold-Nanokompositen}

\begin{tabular}{lcccccr}
\hline In & diesem & Kapitel & \multicolumn{2}{c}{ werden } & Ergebnisse vorgestellt, die & gemeinsam \\
mit & Lena Dyckhoff & im & Rahmen ihrer Bachelorarbeit "Untersuchung \\
der & Wärmeleitfähigkeit & von & Polymer-Nanokompositen“ erhalten wurden.
\end{tabular}

Die Wärmeleitfähigkeit von Polymeren kann durch die Zugabe von metallischen Nanopartikeln erhöht werden. Es müssen jedoch in der Regel mit 30-50 Gew.-\% hohe Anteile an Füllstoff zugesetzt werden, um die Wärmeleitfähigkeit deutlich zu erhöhen. ${ }^{190}$ Bei hohen Füllstoffgehalten bilden sich Netzwerke aus diesen aus, die zu einer deutlichen Erhöhung der Wärmeleitfähigkeit führen. ${ }^{191}$ Eine große Herausforderung stellt auch der thermische Widerstand der Grenzfläche zwischen Matrix und Füllstoff dar. ${ }^{192}$

Um den Grenzflächenwiderstand zu verringern, wurden in dieser Arbeit Gold-Nanopartikel (AuNP) eingesetzt. Aufgrund der hohen Affinität der RAFT-Gruppe zu Goldoberflächen ist es möglich, Polymerketten endständig an die Oberfläche des AuNP zu binden. ${ }^{20} \mathrm{Da}$ auf diese Art eine Interaktion zwischen Nanopartikeln und Polymer, sowie eine gute Dispersion der Partikel in der Matrix erreicht werden kann, könnte dies zur Verringerung des thermischen Widerstandes beitragen. Als Polymer wurde PMMA gewählt, welches schon im vorigen Abschnitt verwendet wurde. Das verwendete PMMA XI wies eine mittlere Molmasse von $M_{\mathrm{n}}=2,8 \cdot 10^{4} \mathrm{~g} \cdot \mathrm{mol}^{-1}$ und eine Dispersität von $Đ=1,08$ auf. Die AuNP wurden gemäß Literaturvorschrift aus Tetrachlorgoldsäure durch Reduktion mit Citrat in wässriger Phase hergestellt. ${ }^{21}$ Zur Funktionalisierung mit dem unpolaren PMMA sollten die Nanopartikel durch Tetraoctylammoniumbromid in eine organische Phase überführt werden, was jedoch zur Aggregation der Nanopartikel führte. Daher wurde ein Lösungsmittelgemisch gesucht, in dem sowohl die Citrat-stabilisierten Nanopartikel, als auch das Polymer löslich ist. Dies konnte durch den Einsatz eines Wasser:THFGemisches (1:3) erreicht werden. Die funktionalisierten Partikel 
wurden durch Transmissionselektronenmikroskopie (TEM) untersucht. In Abbildung 55 ist zu erkennen, dass die funktionalisierten AuNP gut dispergiert vorliegen. Aggregate aus mehreren Nanopartikeln sind nur vereinzelt vorhanden. Es kann geschlussfolgert werden, dass die Funktionalisierung erfolgreich war. Aus den Aufnahmen wurde zudem die Größenverteilung der Nanopartikel bestimmt, wie Kapitel 7.6 zeigt. Es wurde ein Durchmesser von $2 r=(16 \pm 4) \mathrm{nm}$ erhalten.

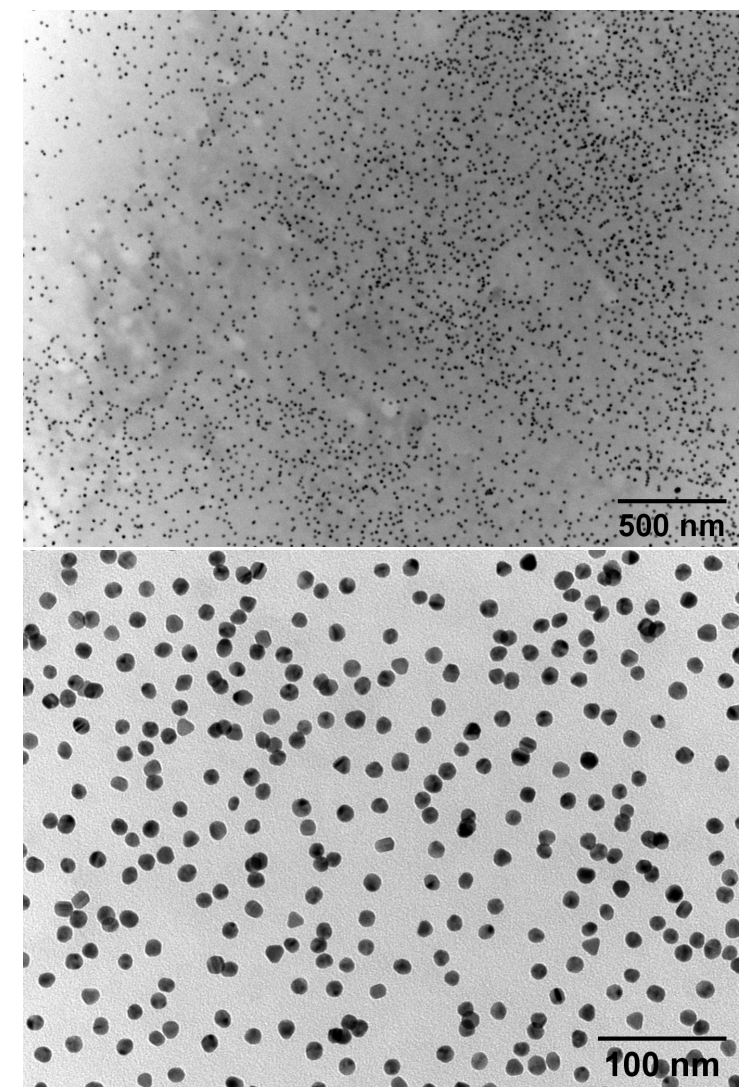

Abbildung 55: TEM-Aufnahmen der PMMA-funktionalisierten AuNP in verschiedenen Vergrößerungen.

Zur Herstellung von Nanokompositen wurden die funktionalisierten AuNP in eine Matrix aus PMMA IX eingebettet. Der Gewichtsanteil an Nanopartikeln betrug dabei 4,8 Gew.-\%. Daraus lässt sich ein Ge- 
wichtsanteil an Gold von 2,3 Gew-\% im Komposit abschätzen wie in Kapitel 7.7 beschrieben ist. Die Wärmeleitfähigkeit der Matrix und des Komposites wurden durch transiente Thermoreflektometrie bestimmt, wie Abbildung 56 zeigt. Es wurde eine Wärmeleitfähigkeit von $0,163 \mathrm{~W} /(\mathrm{K} \cdot \mathrm{m})$ für die Matrix bestimmt. Durch die Zugabe der funktionalisierten Nanopartikel steigt die Wärmeleitfähigkeit um etwa $25 \%$ auf $0,205 \mathrm{~W} /(\mathrm{K} \cdot \mathrm{m})$ an.

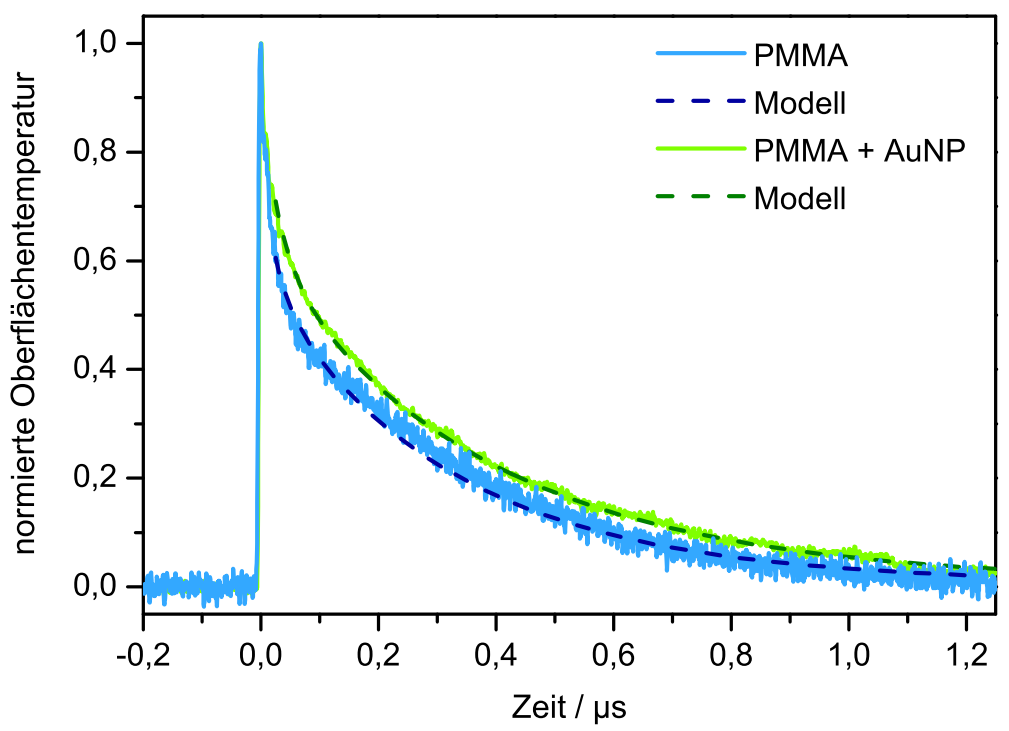

Abbildung 56: Auftragung der normierten Oberflächentemperatur des Kupfers gegen die Zeit nach dem Heizpuls bei den Messungen der transienten Thermoreflektometrie der reinen PMMA-Matrix sowie des Gold-PMMA-Nanokomposites.

Um dieses Resultat einzuordnen, ist ein Vergleich mit Systemen aus der Literatur hilfreich. Ebadi-Dehagani et al. untersuchten ein System aus PP als Matrix und Zinkoxid-Pulver als Füllstoff. Dabei stieg die Wärmeleitfähigkeit des Systems von 0,22 W/(K.m) durch die Zugabe von 5, 10 und 15 Gew.-\% Zinkoxid auf 0,23 W/(K.m), 0,29 W/(K·m) und 0,40 W/(K.m) an. ${ }^{193}$ Zudem hat die Dimensionalität des Füllstoffes einen großen Einfluss darauf, wie sich die Wärmeleitfähigkeit ändert. Dies zeigt ein Vergleich von Kohlenstoff-basierten Füllstoffen mit sehr ähnlicher chemischer Struktur, aber unterschiedlicher Dimensionalität. 
Zu einer Matrix aus Polyvinylidenfluorid wurden 20 Gew.- $\%$ Fullerene (0D), Kohlenstoffnanoröhrchen (1D) und Graphen (2D) gegeben. Die Wärmeleitfähigkeit stieg dabei von 0,189 W/(K·m) auf 0,194 W/(K·m) für Fullerenzugabe, auf 0,604 W/(K·m) bei der Zugabe von Kohlenstoffnanoröhrchen und auf 2,06 W/(K·m) beim Mischen mit Graphen. ${ }^{194}$ Daraus ist deutlich zu erkennen, dass eine höhere Dimensionalität des Füllstoffes die Wärmeleitfähigkeit des Komposites deutlich erhöht. In der Regel ist der Füllstoff so gewählt, dass er eine deutlich höhere Wärmeleitfähigkeit als die Matrix aufweist. Somit trägt ein Füllstoff, der in einer oder zwei Dimensionen eine besondere Ausdehnung hat, stärker zur Wärmeleitfähigkeit bei, als 0-dimensionaler Füllstoff, der keine bevorzugte Transportrichtung für Wärme aufweist. Diese deutliche Zunahme der Wärmeleitfähigkeit wurde auch in der Literatur für Systeme mit 2-dimensionalen Füllstoffen beschrieben. Beispielsweise kann bei Epoxidharzen durch die Zugabe von 5 Gew.-\% Graphen ${ }^{105}$ bzw. Bornitrid $^{190}$ die Wärmeleitfähigkeit um $80 \%$ erhöht werden.

Der Vergleich der erzielten Resultate mit denen der Literatur zeigt, dass die Erhöhung der Wärmeleitfähigkeit im hier untersuchten System trotz der Nutzung eines 0-dimensionalen Füllstoffes und einem geringen Füllstoffanteil mit etwa $25 \%$ außergewöhnlich hoch ist. Mögliche Gründe dafür können in der besonderen Struktur des Systems gefunden werden. Die Grenzfläche zwischen Polymer und Füllstoff weist in der Regel einen großen thermischen Widerstand auf. ${ }^{192}$ Anders als bei den in der Literatur beschriebenen Systemen sind hier jedoch einige Ketten des Matrixpolymers durch die RAFT-Gruppe an die Oberfläche des Füllstoffes gebunden. Diese Bindung könnte dazu führen, dass Phononen-Phononen-Stöße an der Grenzfläche wahrscheinlicher werden und weniger Phononenstreuung auftritt. Dies würde dazu beitragen, den thermischen Widerstand der Grenzfläche zu verringern und kann somit die deutliche Steigerung der Wärmeleitfähigkeit begründen. 
Wärmeleitfähigkeit makromolekularer Systeme

\subsection{Wärmeleitfähigkeit von mikrophasenseparierten Blockcopolymeren}

\begin{tabular}{lcccccr}
\hline In & diesem & Kapitel & werden & Ergebnisse & vorgestellt, die & gemeinsam \\
mit & Lena & Dyckhoff & im & Rahmen ihrer Bachelorarbeit "Untersuchung \\
der & Wärmeleitfähigkeit & von & Polymer-Nanokompositen“ erhalten wurden.
\end{tabular}

Bei den bisher in dieser Arbeit vorgestellten Systemen handelt es sich um Polymere, bei denen keine übergeordnete Strukturierung vorliegt, sondern die Polymere in ihrer amorphen, glasartigen Struktur angeordnet sind. In Blockcopolymeren hingegen kann Mikrophasenseparation auftreten, wodurch das Polymer eine Ordnung aufweist. Der Einfluss einer solchen Strukturierung des Polymers auf die Wärmeleitfähigkeit soll hier am Beispiel von PMMA- $b$-PBA untersucht werden. Durch RAFT-Polymerisation von MMA in Lösung und anschließende RAFT-Polymerisation mit BA konnten vier Blockcopolymere erhalten werden. Die Ansätze wurden dabei so gewählt, dass sich die mittlere Molmasse der Blockcopolymere unterscheidet, der Anteil beider Monomerarten in allen Blockcopolymeren jedoch gleich ist. Dies ist nötig, damit zum einen die Blockcopolymere die gleiche Morphologie der Mikrophasenseparation ausbilden und zum anderen die möglicherweise unterschiedlichen Wärmeleitfähigkeiten der Blöcke keinen Einfluss auf die Gesamtwärmeleitfähigkeit haben.

Tabelle 13: Mittlere Molmasse $M_{\mathrm{n}}$, Dispersität $\oslash$ sowie Stoffmengenanteil an MMA $\chi_{\text {MMA }}$ für BCP VI-IX.

\begin{tabular}{lcccc}
\hline Polymer & $M_{\mathrm{n}} /\left(10^{4} \mathrm{~g} \cdot \mathrm{mol}^{-1}\right)$ & $Đ$ & $I_{\mathrm{MMA}}$ & $\chi_{\mathrm{MMA}}$ \\
\hline BCP VI & 3,79 & 1,22 & 2,29 & 0,43 \\
BCP VII & 6,72 & 1,29 & 2,37 & 0,44 \\
BCP VIII & 6,95 & 1,38 & 2,48 & 0,45 \\
BCP IX & 7,23 & 1,52 & 2,28 & 0,43 \\
\hline
\end{tabular}

Die mittlere molare Masse der Blockcopolymere wurde durch GPC, die Zusammensetzung durch NMR-Spektroskopie bestimmt. Dazu wur- 
den die Integrale der Signale der $\mathrm{O}-\mathrm{CH}_{3}$-Gruppe des MMA $I_{\text {MMA }}$ und der $\mathrm{O}-\mathrm{CH}_{2}$-Gruppe des $\mathrm{BA} I_{\mathrm{BA}}$ verglichen wie Abbildung 57 zeigt. Dabei wurde $I_{\mathrm{BA}}$ auf 2 normiert. Der Stoffmengenanteil an MMA kann dann durch

$$
\chi_{\mathrm{MMA}}=\frac{\frac{I_{\mathrm{MMA}}}{3}}{\frac{I_{\mathrm{MMA}}}{3}+\frac{I_{\mathrm{BA}}}{2}}=\frac{\frac{I_{\mathrm{MMA}}}{3}}{\frac{I_{\mathrm{MMA}}}{3}+1}
$$

berechnet werden. Alle Größen sind in Tabelle 13 zusammengefasst.

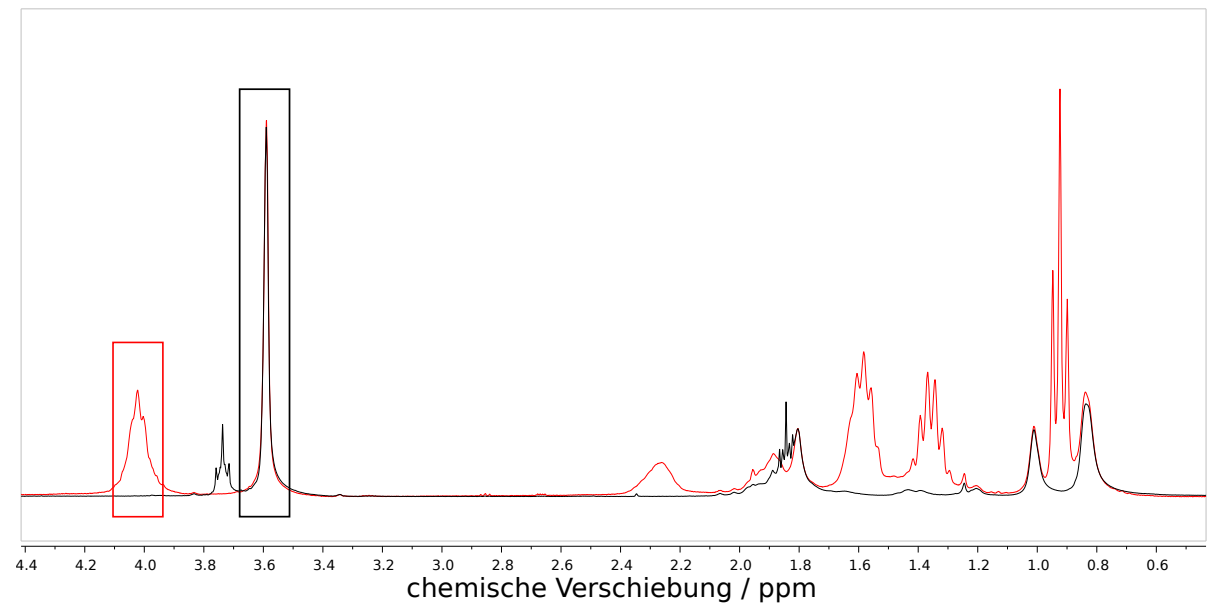

Abbildung 57: ${ }^{1} \mathrm{H}-\mathrm{NMR}$-Spektren von PMMA (schwarz) und PMMA-b-PBA (rot) in $\mathrm{CDCl}_{3}$.

Die Blockcopolymere wurden durch Spincoating einer Lösung in PGMEA auf Silicium-Wafer aufgebracht und sowohl thermisch bei $140^{\circ} \mathrm{C}$ als auch bei Raumtemperatur in Toluol-Atmosphäre getempert. Die Oberfläche der Proben wurde im Anschluss mit dem AFM auf Mikrophasenseparation untersucht. Die Oberflächenprofile sind in Abbildung 58-61 gezeigt. Für alle untersuchten Blockcopolymere ist dabei deutlich zu erkennen, dass Mikrophasenseparation in einer zylindrischen Morphologie vorliegt. Durch Messung der mechanischen Eigenschaften konnte bestimmt werden, dass es sich um Zylinder aus PBA in einer PMMA-Matrix handelt. Die Ausrichtung der Zylinder ist von der Art des Temperns abhängig. 
Wärmeleitfähigkeit makromolekularer Systeme
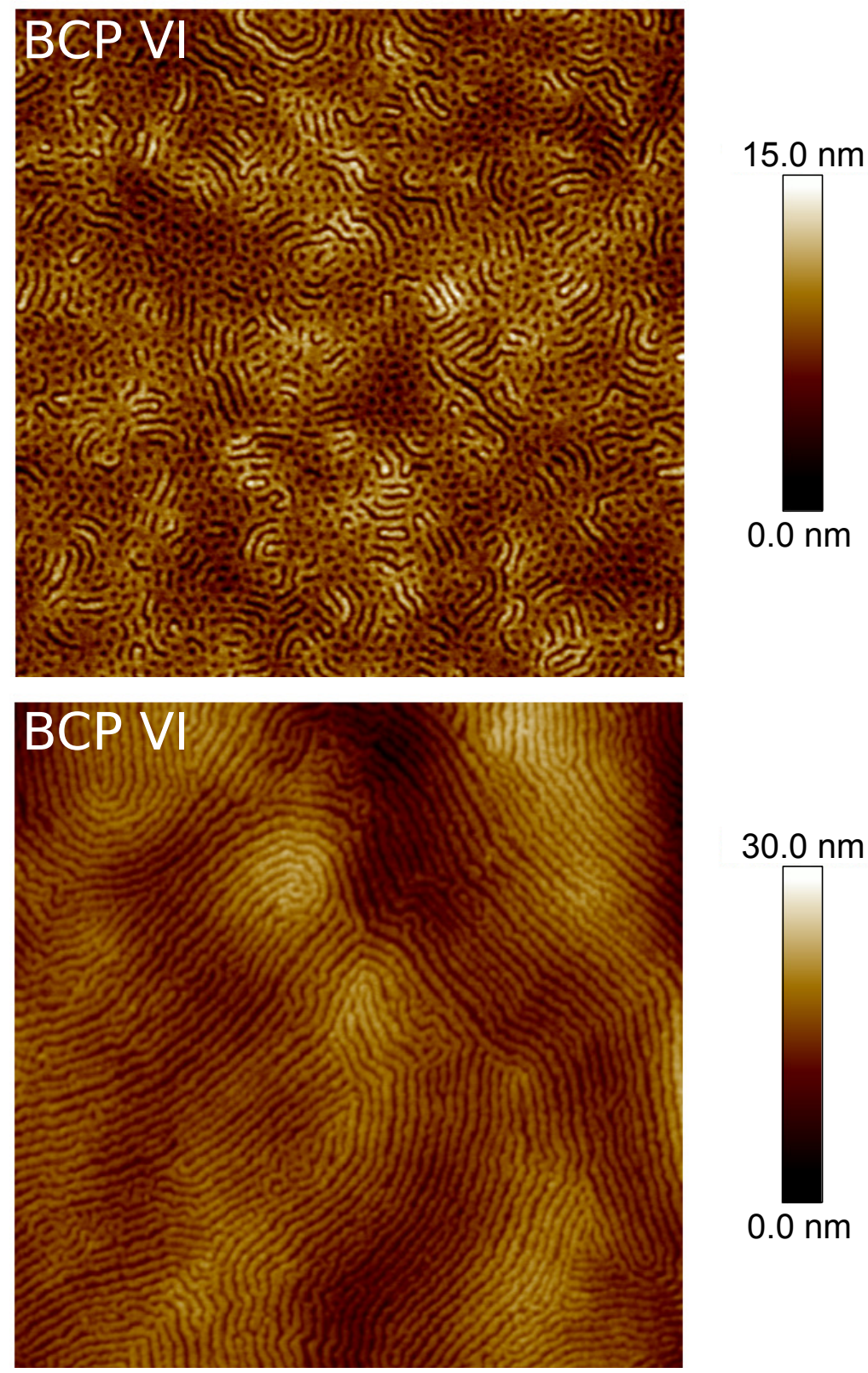

$30.0 \mathrm{~nm}$

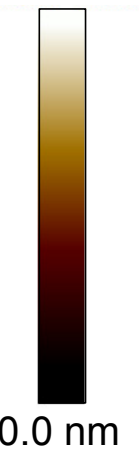

Abbildung 58: AFM-Aufnahmen der Höhenprofile von BCP VI nach thermischem Tempern (oben) und Tempern in Toluol-Atmosphäre (unten). Die Proben wurden auf Si-Wafer gespincoatet. Ausschnitt: $2 \times 2 \mu \mathrm{m}$, Auflösung: $512 \times 512$ Pixel. 
Wärmeleitfähigkeit makromolekularer Systeme
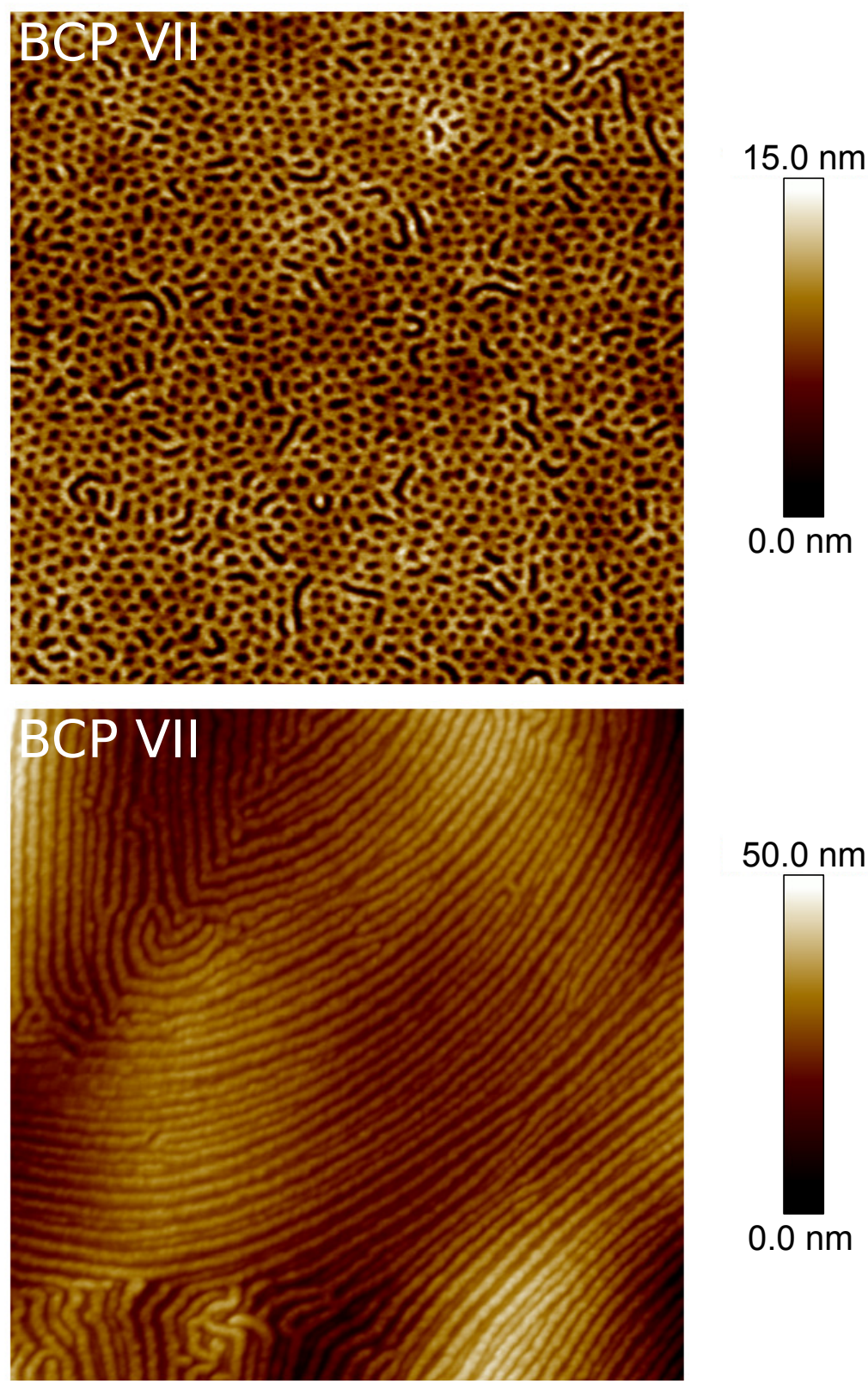

Abbildung 59: AFM-Aufnahmen der Höhenprofile von BCP VII nach thermischem Tempern (oben) und Tempern in Toluol-Atmosphäre (unten). Die Proben wurden auf Si-Wafer gespincoatet. Ausschnitt: $2 \times 2 \mu \mathrm{m}$, Auflösung: $512 \times 512$ Pixel. 
Wärmeleitfähigkeit makromolekularer Systeme

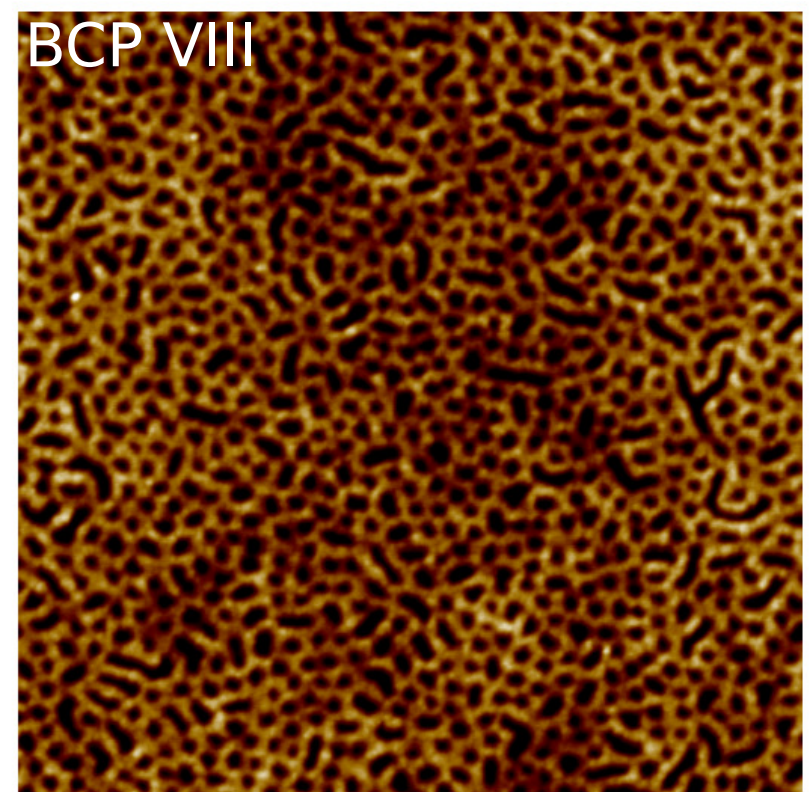

\section{$15.0 \mathrm{~nm}$}
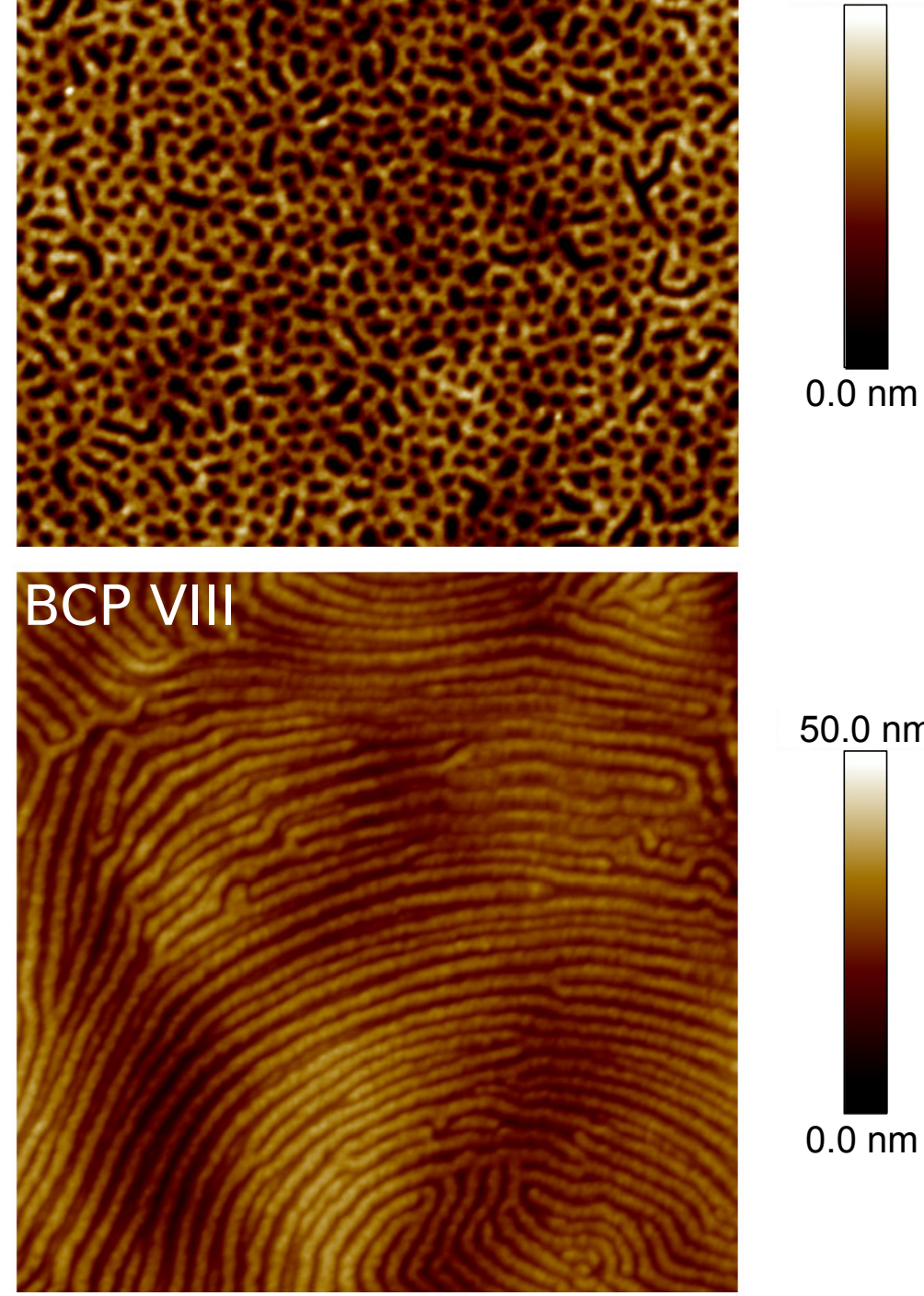

$50.0 \mathrm{~nm}$

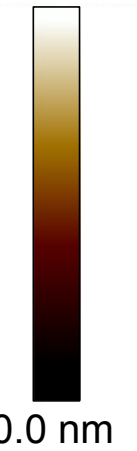

Abbildung 60: AFM-Aufnahmen der Höhenprofile von BCP VIII nach thermischem Tempern (oben) und Tempern in Toluol-Atmosphäre (unten). Die Proben wurden auf Si-Wafer gespincoatet. Ausschnitt: $2 \times 2 \mu \mathrm{m}$, Auflösung: $512 \times 512$ Pixel. 
Wärmeleitfähigkeit makromolekularer Systeme
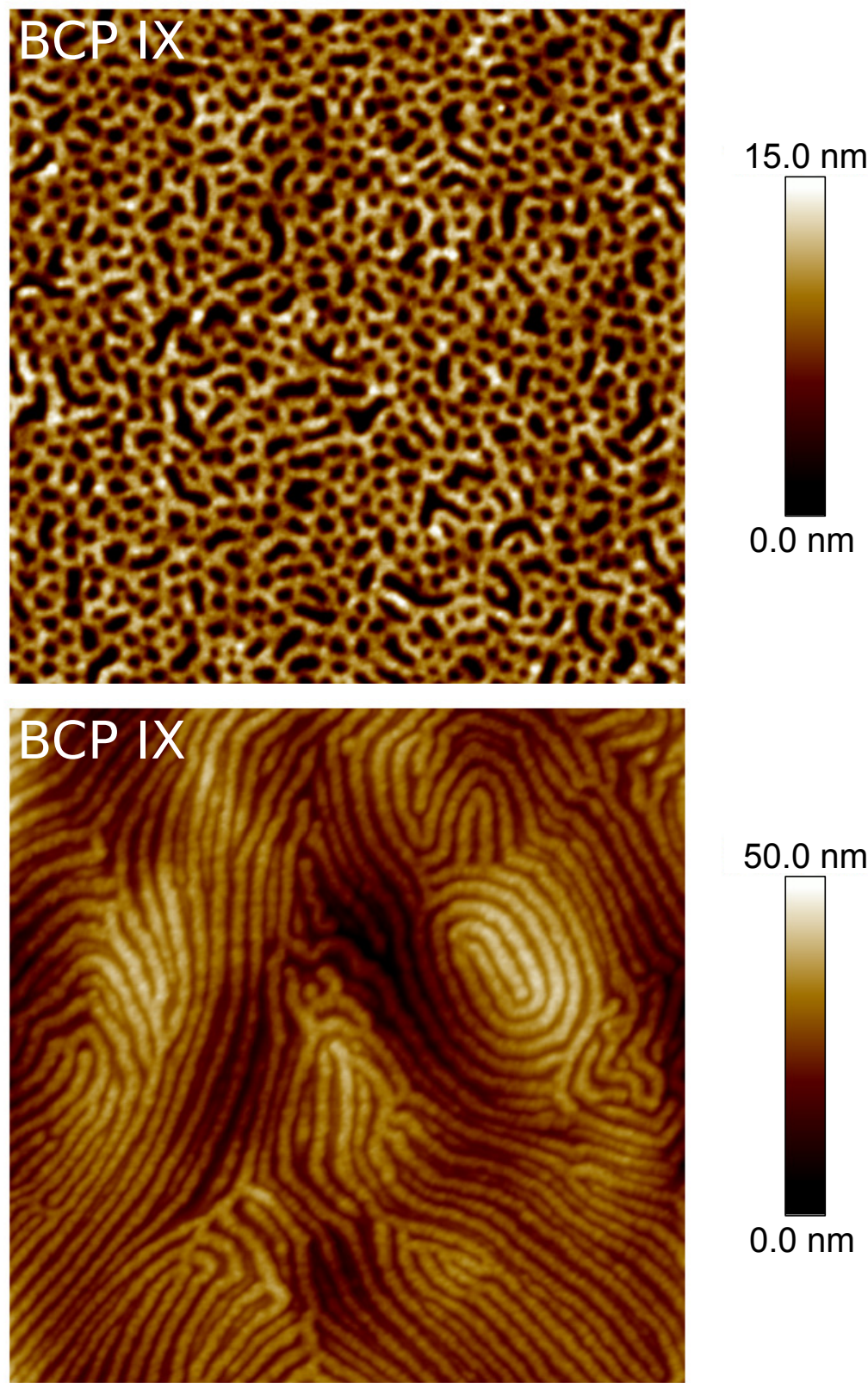

Abbildung 61: AFM-Aufnahmen der Höhenprofile von BCP IX nach thermischem Tempern (oben) und Tempern in Toluol-Atmosphäre (unten). Die Proben wurden auf Si-Wafer gespincoatet. Ausschnitt: $2 \times 2 \mu \mathrm{m}$, Auflösung: $512 \times 512$ Pixel. 
Nach thermischem Tempern sind die Zylinder senkrecht zur Probenoberfläche angeordnet, während sie nach Lösungsmitteltempern entlang der Oberfläche ausgerichtet sind. Zudem ist zu erkennen, dass der Abstand der Zylinder mit der Molmasse des Polymers ansteigt, wie Tabelle 14 zeigt.

Tabelle 14: Zylinderabstand für die mikrophasenseparierten BCP VI-IX.

\begin{tabular}{lcccc}
\hline & BCP VI & BCP VII & BCP VIII & BCP IX \\
\hline Stehend & $35,8 \mathrm{~nm}$ & $57,0 \mathrm{~nm}$ & $58,4 \mathrm{~nm}$ & $69,2 \mathrm{~nm}$ \\
Liegend & $34,3 \mathrm{~nm}$ & $41,7 \mathrm{~nm}$ & $46,7 \mathrm{~nm}$ & $52,3 \mathrm{~nm}$ \\
\hline
\end{tabular}

Die unterschiedliche Ausrichtung der Zylinder ermöglicht eine Messung der Wärmeleitfähigkeit in unterschiedliche Richtungen, da die Messung der Wärmeleitfähigkeit stets senkrecht zur Probenoberfläche stattfindet. Die Wärmeleitfähigkeiten der Proben wurde durch TTR ermittelt.

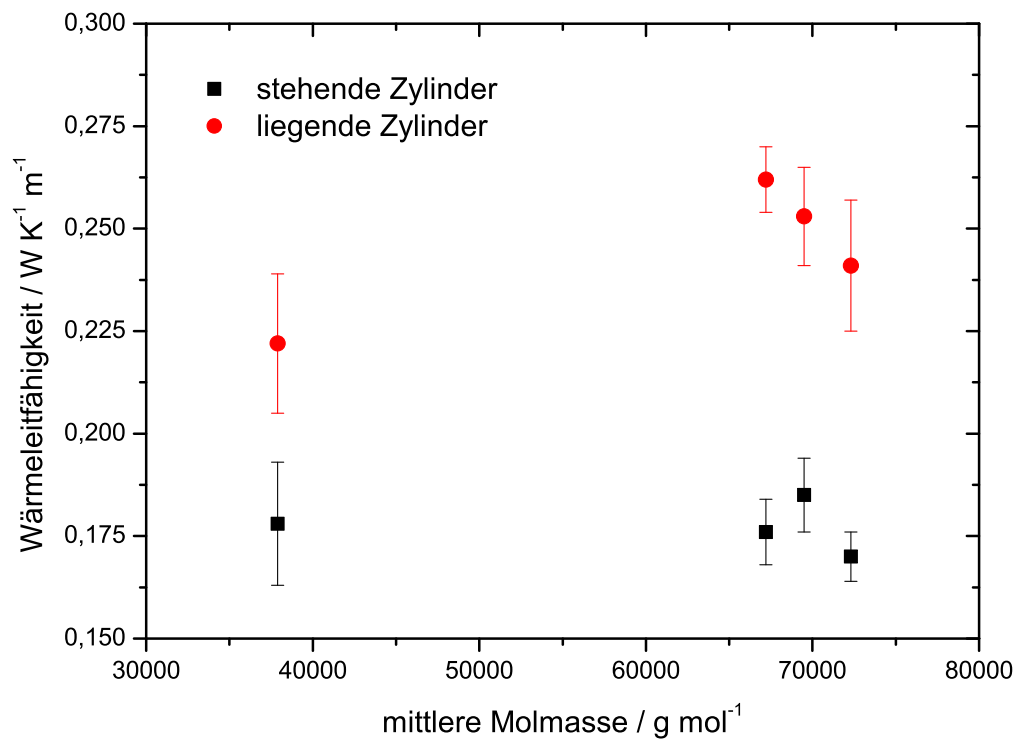

Abbildung 62: Wärmeleitfähigkeit der Blockcopolymere BCP VI-IX in Abhängigkeit von der Ausrichtung der Zylinder und der mittleren Molmasse. 
Wie in Abbildung 62 dargestellt ist, konnte auch hier keine Abhängigkeit der Wärmeleitfähigkeit von der Kettenlänge des Polymers beobachtet werden. Jedoch ist eine deutliche Anisotropie der Wärmeleitfähigkeit zu erkennen. Für die stehenden Zylinder liegt die Wärmeleitfähigkeit mit durchschnittlich 0,177 W/(K·m) in der Größenordnung der für PMMA bestimmten Werte. Im Falle der liegenden Zylinder wurde eine etwa $40 \%$ höhere Wärmeleitfähigkeit von $0,245 \mathrm{~W} /(\mathrm{K} \cdot \mathrm{m})$ ermittelt. Da es sich jeweils um das gleiche Blockcopolymer handelt, können die unterschiedlichen Wärmeleitfähigkeiten auf die Unterschiede in der Struktur zurückgeführt werden. In der Nähe der Grenzfläche zwischen den beiden Phasen sind die Ketten zur Minimierung der Grenzflächenenergie gestreckt. Liegt diese gestreckte Kette in Transportrichtung der Wärme vor, so führt dies dazu, dass der Wärmetransport beschleunigt wird, da der Transport von Wärme entlang einer Kette schnell erfolgen kann. Dies ist beim Wärmetransport in den Systemen mit liegenden Zylindern der Fall, wodurch die erhöhte Wärmeleitfähigkeit begründet werden kann. Bei stehenden Zylindern liegen alle gestreckten Ketten in der Ebene, die senkrecht zur Transportrichtung ausgerichtet ist. Daher leisten diese gestreckten Segmente keinen Beitrag zur Wärmeleitfähigkeit, sodass diese in der Größenordnung eines amorphen Polymers liegt.

\subsection{Fazit und weitere Forschungsperspektiven}

PAzoPMA, PMMA-Homopolymere, Komposite aus PMMA und AuNP sowie PMMA-b-PBA-Blockcopolymere wurden durch TTR auf ihre Wärmeleitfähigkeitseigenschaften untersucht. Dabei wurden unterschiedliche Aspekte des Wärmetransportes in Polymeren und Nanokompositen beleuchtet.

Im photoschaltbaren Polymer PAzoPMA wurde der Einfluss des Schaltzustandes der Azobenzol-Seitengruppe im Polymer auf die Wärmeleitfähigkeit untersucht. Es konnte gezeigt werden, dass die Wärmeleitfähigkeit von PAzoPMA durch das Auslösen des Photoschalters um etwa $60 \%$ sinkt. Ob diese Änderung reversibel ist, 
Wärmeleitfähigkeit makromolekularer Systeme

kann Thema zukünftiger Forschung sein. Um herauszufinden, ob die $\pi-\pi$-Wechselwirkungen Ursprung der hohen Wärmeleitfähigkeit von PAzoPMA sind, könnten Ansätze gewählt werden, bei denen die Ausbildung solcher Wechselwirkungen zwischen verschiedenen Seitengruppen unterdrückt wird. Dies kann möglicherweise durch das Einführen von sterisch anspruchsvollen Substituenten am Azobenzol erreicht werden. Zudem können die photoschaltbaren Polymere PAzoMA, PAzoHMA und PAzoUMA mit dem hier bereits untersuchten PAzoPMA verglichen werden, um herauszufinden, inwiefern die chemische Struktur der Seitenkette einen Einfluss auf die Änderung der Wärmeleitfähigkeit beim Schalten hat. Aus den Resultaten können möglicherweise Rückschlüsse über den Grund der Wärmeleitfähigkeitsänderung gezogen werden.

Am Beispiel von PMMA wurde die Wärmeleitfähigkeit von amorphen Polymeren in Abhängigkeit von der Kettenlänge untersucht. Es konnte gezeigt werden, dass die Wärmeleitfähigkeit für mittlere Molmassen des Polymers von 3600 bis $160000 \mathrm{~g} \cdot \mathrm{mol}^{-1}$ konstant ist, wie es auch Simulationen für große Kettenlängen prognostizierten. ${ }^{188}$ Der vorausgesagte lineare Anstieg der Wärmeleitfähigkeit für kurze Ketten konnte nicht beobachtet werden, sodass dieser wahrscheinlich unterhalb von $3600 \mathrm{~g} \cdot \mathrm{mol}^{-1}$ beginnt. Aufgrund der niedrigen Glasübergangstemperatur von PMMA bei solch kurzen Ketten ist eine Messung der Wärmeleitfähigkeit bei Raumtemperatur nicht möglich, da die Glasübergangstemperatur durch den Heizpuls überschritten werden würde. Eine Messreihe bei tiefen Temperaturen könnte die Bestimmung der Wärmeleitfähigkeit von kurzen Ketten ermöglichen, sodass der lineare Anstieg der Wärmeleitfähigkeit auch experimentell nachgewiesen werden könnte. Zudem könnten andere einfache, lineare Polymere untersucht werden um festzustellen, welchen Einfluss die Struktur des Polymers darauf hat, wann der lineare Anstieg abflacht und gegen den Grenzwert für sehr lange Ketten strebt.

Zur Herstellung von Polymernanokompositen wurden Citratstabilisierte Goldnanopartikel mit PMMA funktionalisiert, sodass 
diese gut dispergiert vorlagen. Anschließend wurden die Partikel in eine PMMA-Matrix eingelagert und die Wärmeleitfähigkeit bestimmt. Durch die Zugabe von 4,8 Gew.-\% funktionalisierter Goldnanopartikel konnte die Wärmeleitfähigkeit um $25 \%$ gesteigert werden. Eine Studie mit Proben, die unterschiedliche Füllstoffgehalte an Goldnanopartikeln enthalten, könnte Aufschluss darüber geben, ob es wie bei vergleichbaren Systemen einen Schwellenwert gibt, oberhalb von dem die Wärmeleitfähigkeit nicht mehr linear, sondern exponentiell zunimmt. ${ }^{191}$ Zudem können durch Verwendung von Polymer mit unterschiedlichen Kettenlänge zur Funktionalisierung Nanopartikel mit unterschiedlichen Beladungsdichten an Polymer erhalten werden. Eine steigende Wärmeleitfähigkeit mit höherer Beladungsdichte an Polymer würde die in dieser Arbeit aufgestellte These, dass die Funktionalisierung den thermischen Widerstand der Füllstoff-Matrix-Grenzfläche verringert, bestätigen. Auch ein Vergleich mit funktionalisierten 1Doder 2D-Füllstoffen wäre interessant. Durch die Funktionalisierung des Füllstoffes ließe sich in solchen Systemen die ohnehin schon sehr gute Wärmeleitfähigkeit möglicherweise weiter erhöhen, sodass die Leistungsfähigkeit von Polymernanokompositen weiter gesteigert werden könnte.

Die Auswirkungen einer Strukturierung im Polymer wurde anhand des mikrophasenseparierenden Blockcopolymers PMMA- $b$-PBA untersucht. Durch unterschiedliche Temperverfahren (thermisch und durch Lösungsmitteldampf) wurde eine zylindrische Morphologie der Mikrophasenseparation erhalten, welche sich jedoch in der Ausrichtung der Zylinder unterscheidet. Dies ermöglichte die richtungsabhängige Messung der Wärmeleitfähigkeit. Dabei konnte eine Anisotropie der Wärmeleitfähigkeit des Blockcopolymers festgestellt werden. Sind die Zylinder entlang der Probenoberfläche angeordnet, so ist die Wärmeleitfähigkeit etwa $40 \%$ höher, als bei senkrecht zur Oberfläche angeordneten Zylindern. Durch eine Untersuchung von PMMA-b-PBA mit anderer Morphologie können die hier aufgestellen Thesen für die Anisotropie der Wärmeleitfähigkeit überprüft werden. Ein Blockco- 
polymer mit lamellarer Morphologie, bei der die Lamellen parallel zur Substratoberfläche angeordnet sind, sollte demnach eine sehr hohe Wärmeleitfähigkeit aufweisen. Ebenso kann ein Vergleich mit dem ungetemperten Blockcopolymer weitere Informationen liefern, inwiefern die Strukturierung der Probe einen Einfluss auf die Wärmeleitfähigkeit des Polymers ausübt. 
Ausblick

\section{Ausblick}

Die cis $\rightarrow$ trans-Isomerisierung in den Azobenzol-basierten Monomeren AzoMA, AzoPMA, AzoHMA und AzoUMA, sowie in den zugehörigen Polymeren wurde durch zeitaufgelöste UV/Vis-Spektroskopie untersucht. Der Einfluss von Temperatur, Lösungsmittel und Linkerlänge auf den Geschwindigkeitskoeffizienten der Isomerisierungsreaktion wurde analysiert, sowie Unterschiede im Verhalten von Monomeren und Polymeren herausgestellt.

Eine Untersuchung der Abhängigkeit der Isomerisierungsgeschwindigkeit von der Kettenlänge des Polymers kann in Zukunft wichtige Informationen $\mathrm{zu}$ diesem System liefern. Insbesondere sehr kurze Ketten sind von Interesse, um die Faktoren, die für den Unterschied des Verhaltens von Monomer und Polymer verantwortlich sind, aufzuschlüsseln. Sowohl das Auftreten eines kooperativen Effektes, der zu einer Beschleunigung der Isomerisierung führt, als auch der Einfluss des End-zu-End-Abstandes im Polymerknäul sollten im Bereich kurzer Ketten zu beobachten sein. Anhand des Verhaltens langer Ketten kann untersucht werden, ob die genannten Effekte linear oder exponentiell mit der Kettenlänge zunehmen, oder sich einem Grenzwert annähern. Auch das Auftreten von Schwellenwerten, an denen sich das Verhalten schlagartig ändert, ist möglich.

Aus den photoschaltbaren Monomeren konnten durch Copolymerisation mit BA Blockcopolymere hergestellt werden. Die Oberflächenstruktur von Dünnfilmen dieser Polymere wurde durch AFM untersucht. Es wurden Methoden vorgestellt, um verschiedene Strukturparameter der selbstangeordneten mikrophasenseparierten Struktur gezielt einzustellen.

Die Möglichkeit, dieses nanostrukturierte Material maßschneidern zu können, eröffnet neue Perspektiven für die künftige Forschung. Es kann untersucht werden, wie sich die spezifischen Strukturparameter auf z.B. mechanische oder thermische Eigenschaften des Materials auswirken. Zudem ist zu erwarten, dass das System anisotrope Ei- 
genschaften aufweist, die sich durch den vorhandenen Photoschalter möglicherweise zwischen zwei Zuständen schalten lassen.

In Lösung konnten aus PAzoPMA- $b$-PDMAEMA photoschaltbare Aggregate hergestellt werden, die mit Kristallviolett beladen wurden. Durch Bestrahlung mit UV-Licht lösen sich die Aggregate reversibel auf. Dies kann als Prototyp für ein System zur Wirkstoffverabreichung dienen, bei dem Medikamente in photoschaltbare Aggregate eingeschlossen werden, um diese durch Bestrahlung an der gewünschten Stelle gezielt freizusetzen.

Durch TTR wurden PMMA, PAzoPMA, PMMA- $b$-PBA sowie ein Komposit aus PMMA und funktionalisierten AuNP auf ihre Wärmeleitfähigkeitseigenschaften untersucht. Die Abhängigkeit der Kettenlänge eines Polymers auf die Wärmeleitfähigkeit wurde am Beispiel von PMMA untersucht. Es wurde gezeigt, dass die Wärmeleitfähigkeit für Proben zwischen $3,6 \cdot 10^{3}$ und $1,6 \cdot 10^{5} \mathrm{~g} / \mathrm{mol}$ keine Abhängigkeit von der mittleren Molmasse des Polymers aufweist. Eine Untersuchung von Polymeren mit kürzeren Ketten könnte Aufschluss darüber geben, ob der durch Simulationen vorausgesagte Abfall der Wärmeleitfähigkeit für kleine Molmassen auch experimentell $\mathrm{zu}$ beobachten ist.

Für PAzoPMA konnte die Wärmeleitfähigkeit durch Bestrahlung mit UV-Licht um $60 \%$ gesenkt werden. Somit ist die Nutzung von Photoschaltern auch in anderen makromolekularen Systemen zur Schaltung der Wärmeleitfähigkeitseigenschaften vielversprechend.

Das Nanokomposit aus PMMA und AuNP zeigte im Vergleich zur reinen Matrix eine unerwartet große Wärmeleitfähigkeit, die deutlich über der von Kompositen mit unfunktionalisierten Füllstoffen liegt. Darauf aufbauend könnten auch Füllstoffe mit einer höheren Dimensionalität wie z.B. Graphen oder Kohlenstoffnanoröhrchen mit Polymer funktionalisiert werden, um die Wärmeleitfähigkeit von Nanokompositen weiter zu steigern.

Am Beispiel von mikrophasenseparierten Blockcopolymeren mit zylindrischer Morphologie wurde gezeigt, dass die Wärmeleitfähigkeit in ei- 
nem nanostrukturierten Material anisotrop ist. Ein Vergleich mit einem Blockcopolymer in ungeordnetem Zustand oder mit einer anderen Morphologie der Mikrophasenseparation könnte weitere Informationen liefern, inwiefern die Strukturierung des Materials die Wärmeleitfähigkeit beeinflusst. 


\section{Experimentalteil}

\subsection{Chemikalien}

Alle Chemikalien wurden von den Herstellern ABCR, Acros, Deutero, Merck, Roth, VWR und Sigma Aldrich erworben und ohne weitere Aufreinigung eingesetzt. Sie wiesen eine Reinheit von $95 \%$ oder höher auf. Die eingesetzten Lösungsmittel hatten pro analysi-Qualität. Die Lösungsmittel für die Säulenchromatografie sowie für die Aufreinigung durch Zentrifugation hatten technische Qualität.

\subsection{Monomere}

BA (Sigma Aldrich, $\geq 99 \%$, stabilisiert mit Hydrochinonmonomethylether), DMAEMA (Sigma Aldrich, $\geq 99 \%$, stabilisiert mit Hydrochinonmonomethylether) und MMA (Sigma Aldrich, $99 \%$, stabilisiert mit Hydrochinonmonomethylether) wurden vor der Verwendung über basischem Aluminiumoxid oder Inhibitor Remover filtriert. Zudem wurde vor dem Einsatz gelöster Sauerstoff durch Spülen mit einem Argonstrom entfernt.

\subsection{Initiatoren}

2,2'-Azobis(2-methylpropionitril) und 1,1'-Azobis(cyclohexancarbonitril) wurden aus Methanol umkristallisiert, im Vakuum getrocknet und bei $-18{ }^{\circ} \mathrm{C}$ gelagert.

\subsection{RAFT-Agens}

Das verwendete RAFT-Agens 4-Cyano-4-[(dodecylsulfanylthiocarbonyl)sulfanyl]-pentansäure (Sigma Aldrich, $97 \%$ ) wurde ohne weitere Aufreinung eingesetzt. Es eignet sich gut zur Polymerisation von Acrylaten und Methacrylaten. ${ }^{8-12}$ 


\subsection{Mess- und Analysegeräte}

\section{Rasterkraftmikroskop (AFM)}

AFM-Messungen wurden mit einem Multimode 8 AFM, welches mit einem NanoScope $V$ Kontroller von der Firma Veeco/Digital Instruments ausgestattet ist, durchgeführt. Für die Messungen wurde das Programm Nanoscope Version 8.15 und der Modus ScanAsystHR in Air verwendet. Als Cantilever wurde der SCANASYST-AIR-HR (Siliciumspitze auf Nitridarm, Resonanzfrequenz $130 \pm 30 \mathrm{kHz}$, Federkonstante 0,4 N/m, Spitzenradius 0,995 nm, Bruker AFM Probes) eingesetzt. Zur Schonung von Material und Probe wurde bei den Messungen wurde die Krafteinwirkung des Cantilevers auf die Oberfläche vom Messprogramm automatisch bestimmt.

Die Datenanalyse erfolgte durch das Programm NanoScope Analysis Version 1.40 .

\section{Gelpermeationschromatografie}

Als System zur Bestimmung von Molmassenverteilungen durch GPC wurde ein SEC Analysis System 1260 Infinity von PSS Agilent verwendet. Es besteht aus einer HPLC-Pumpe (PSS Agilent Technologies 1260 Iso Pump G1310B), einem Autosampler (Agilent 1260 ALS G1329B), einem Injektor (Agilent 1260 ALS Injektor), einer Vorsäule (PSS SDV, $8 \times 50 \mathrm{~mm}$, Partikelgröße: $5 \mu \mathrm{m}$ ), drei Trennsäulen (PSS SDV, $8 \times 50 \mathrm{~mm}$, Partikelgröße: $5 \mu \mathrm{m}$, Porengröße: $10^{6} \AA, 10^{5} \AA$ und $10^{3} \AA$ ) und den Detektoren. Als Detektoren wurden ein Brechungsindex-Detektor (PSS Agilent Technologies 1260 RID) und ein UV-Detektor (PSS Agilent Technologies $1260 V W D V L, \lambda=325 \mathrm{~nm}$ ) verwendet. Das Laufmittel bestand aus THF (HPLC-Grad) mit Toluol (> 99,7 \%, trocken) als internem Standard bei einer Fließgeschwindigkeit von $1,0 \mathrm{~mL} / \mathrm{min}$ und $35^{\circ} \mathrm{C}$. Die Proben wurden vor der Messung durch einen $450 \mathrm{~nm}$ PTFE-Filter filtriert.

Das System wurde mit eng verteilten PolymethylmethacrylatStandards kalibiriert. Die Mark-Houwink-Parameter betrugen 
$\mathrm{K}=0,01298$ und $\alpha=0,688$. Zur Auswertung wurde die Software PSS WinGPC verwendet.

\section{NMR-Spektroskopie}

Die NMR-Spektren wurden mit einem Avance DRX 300 der Firma Bruker aufgenommen. Die chemische Verschiebung in ppm bezieht sich auf Tetramethylsilan. Als Referenz wurde jeweils das verwendete Lösungsmittel verwendet. Für die Signalmultiplizitäten wurden die folgenden Abkürzungen verwendet: $\mathrm{s}=$ Singulett, $\mathrm{d}=$ Dublett, $\mathrm{t}=$ Triplett, $\mathrm{q}=$ Quartett, quint $=$ Quintett, sext $=$ Sextett, $\mathrm{m}=$ Multiplett. Bei mehreren Kopplungen werden die Kopplungen nach der Größe der Kopplungskonstante sortiert. Als Lösungsmittel wurde $\mathrm{CD}_{2} \mathrm{Cl}_{2}$ verwendet. Das Signal des Lösungsmittels wurde der Verschiebung 5,32 ppm zugeordnet.

\section{Dynamische Differenzkalorimetrie}

Die Glasübergangstemperaturen wurden durch dynamische Differenzkalorimetrie an einer DSC 214 Polyma der Firma Netzsch bestimmt. Die Proben wurden dazu in einen Aluminiumtiegel mit Deckel (Concavus, Firma Netzsch) gefüllt. Das Temperaturprogramm heizt mit einer Rate von $10 \mathrm{~K} / \mathrm{min}$ zunächst von $30^{\circ} \mathrm{C}$ auf $170{ }^{\circ} \mathrm{C}$, kühlt dann von $170{ }^{\circ} \mathrm{C}$ auf $0{ }^{\circ} \mathrm{C}$ ab und heizt zuletzt erneut von $0{ }^{\circ} \mathrm{C}$ auf $170{ }^{\circ} \mathrm{C}$. Nach jedem Aufheizen/Abkühlen wurde die Temperatur für 5 min konstant gehalten.

\section{Massenspektrometrie}

ESI-Massenspektrometrie wurde mit einem Daltonik ESI-ToF-MS der Firma Bruker von der Zentralen Analytik des Institutes für Organische Chemie der Georg-August-Universität Göttingen durchgeführt. 


\section{Probenpräparation zur AFM-Untersuchung}

Als Substrat für die AFM-Messungen wurden quadratische Silicium (111)-Wafer mit einer Seitenlänge von $10 \mathrm{~mm}$ der Firma Plano GmbH genutzt. Nach Spülen mit Aceton und THF wurden diese in einem Plasmaofen (Typ ZEPTO, Firma electronic diener, Plasma-SurfaceTechnology) gereinigt.

$10 \mu \mathrm{L}$ einer Lösung des Polymers in Propylenglykolmonomethyletheracetat (1-10 wt $\%$ ) wurden auf den Silicium-Wafer gegeben und dieser für eine Minute bei 2000 rpm mittels eines Spincoaters (WS-650MZ-23NPP von Laurell) rotiert.

Die Proben wurden anschließend bei erhöhter Temperatur oder unter Lösungsmittelatmosphäre bei bei Raumtemperatur getempert.

\section{Schalten der funktionellen Gruppe}

Zum Isomerisieren der funktionellen Azobenzol-Gruppe wurden Proben in Lösung 30 Minuten, Proben im Festkörper 60 Minuten mit UVLicht bestrahlt. Die verwendete UV-Lampe der Firma Herolab mit einer Leistung von $8 \mathrm{~W}$ erzeugte Strahlung mit einer Wellenlänge von $366 \mathrm{~nm}$.

\section{Berechnung von Dipolmomenten}

Die Dipolmomente wurden mit dem Programm TINKER 8 unter Verwendung eines MM3-Forcefields von Niklas Frerichs berechnet. ${ }^{195}$ Die verwendeten Strukturen wurden mit dem Programm Chem3D 16.0 erstellt und die Struktur in einem MM2-Forcefield auf ein energetisches Minimum optimiert.

\section{UV/Vis-Spektroskopie}

Die UV/Vis-Spektren wurde mit einem Cary 300 Scan Photospektrometer mit Quarz-Küvetten von HellmaAnalytics mit einer Schichtdicke von $10 \mathrm{~mm}$ aufgenommen. Das Spektrum des reinen Lösungsmittels 
wurde als Hintergrund aufgenommen und von den gemessenen Spektren subtrahiert.

\section{Transmissionselektronenmikroskopie (TEM)}

Die Aufnahmen der Au-Nanopartikel wurden an einem Transmissionselektronenmikroskop (Philips CM 12) mit einer CCD-Kamera (Olympus, 1376 x 1032 Pixel) gemeinsam mit Yingying Cai, Darius Rohleder und Lena Dyckhoff durchgeführt. Es lag eine Beschleunigungsspannung von $120 \mathrm{kV}$ an, der Emissionsstrom betrug 3-4 $\mu \mathrm{A}$. Für die Kondensatorlinse wurde eine $50 \mu \mathrm{m}$ Blende, die Kontrastblende wies einen Durchmesser von $20 \mu \mathrm{m}$ auf. Die Proben wurden zuvor durch Auftropfen einer Lösung des Probenmaterials auf mit einem amorphen Kohlenstofffilm (10-15 nm) überzogenes Plano 200 mesh-Kupfernetz. Das Lösungsmittel wurde dabei langsam entfernt.

\section{Transiente Thermoreflektometrie (TTR)}

Die Messungen zur transienten Thermoreflektrometrie wurden am I. Physikalischen Institut der Georg-August-Universität Göttingen vom Autor der Arbeit, sowie von Dr. Henning Ulrichs, Daniel Metternich, Dennis Meyer und Roland Potthast durchgeführt. $10 \mu l$ einer 5 Gew.-\% Lösung der Probe wurde dazu durch Spincoating auf einen quadratischen Silicium (111)-Wafer mit einer Seitenlänge von $10 \mathrm{~mm}$ der Firma Plano GmbH aufgebracht und anschließend im Hochvakuum mit $50 \mathrm{~nm}$ Kupfer bedampft. Die Messung erfolgte mit einem Heizlaser der Firma Coherent mit einer Wellenlänge von $\lambda=515 \mathrm{~nm}$ und einem Dauerstrichlaser mit einer Wellenlänge von $\lambda=643 \mathrm{~nm}$ zur Messung der Reflektivität. Die Intensität des Dauerstrichlasers wurde mit einer schnellen Si-Photodiode und einem Oszilloskop aufgezeichnet. Dabei wurde für jede Messung über mindestens 65.000 Heizpulse gemittelt. 
Bestimmung der Wärmeleitfähigkeit aus den Messungen der transienten Thermoreflektometrie

Zur Berechnung der Wärmeleitfähigkeit wurden die ReflektivitätsZeit-Kurven aus der TTR mit dem Programm TTR sim (erstellt von Dr. Henning Ulrichs, Dennis Meyer, Daniel Metternich und Roland Potthast) basierend auf einem Modell für 1-D-Wärmetransport in geschichteten Systemen nach [196] verwendet.

\section{Ellipsometrie}

Zur Bestimmung der Schichtdicke von Polymerdünnfilmen wurde ein Accurion EP4 Imaging Ellipsometer verwendet, welches mit einem Lichtmikroskop mit 10-facher Vergrößerung der Firma Nikon ausgestattet ist. Die Messung erfolgte an zwei Stellen der Probe mit 12 Wellenlängen zwischen 450 und $800 \mathrm{~nm}$ und wurde zweifach durchgeführt. Das Gerät wurde mit dem Programm EP4 Control 3.2.0.2 gesteuert. Die Auswertung wurde mit dem Programm EP4 Model 3.4.2.0 durchgeführt. Dabei wurde ein Modell aus unendlich dickem Si (111), $10 \mathrm{~nm} \mathrm{SiO}$, der Probesubstanz und einer unendlich dicken Schicht Luft verwendet um die Schichtdicke und den Brechungsindex der Probe zu modellieren. 


\subsection{Synthesen der photoschaltbaren Monomere}

Synthese von 3-(4-(Phenyldiazenyl)phenoxy)propan-1-ol<smiles>OCCCBr</smiles>

$198.23 \mathrm{~g} / \mathrm{mol}$

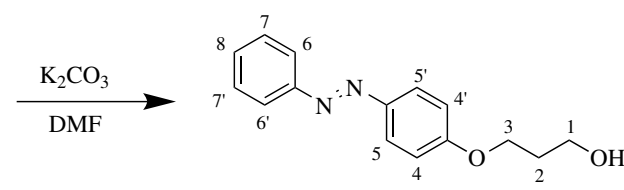

$256.31 \mathrm{~g} / \mathrm{mol}$

4-Phenylazophenol (24,0 g, $120 \mathrm{mmol}, 1,00$ Äq.), Kaliumcarbonat (24,9 g, 180 mmol, 1,50 Äq.) und eine Spatelspitze Kaliumiodid wurden in DMF (200 ml) gelöst und für eine Stunde gerührt. Es wurde 3Brompropanol (25,0 g, $181 \mathrm{mmol}, 1,51$ Äq.) langsam zugetropft und das Reaktionsgemisch über Nacht refluxiert. Das Lösungsmittel wurde im Vakuum entfernt. Der Feststoff wurde mit Diethylether aufgenommen und filtriert. Der Rückstand wurde mit Diethylether gewaschen. Das Lösungsmittel des Filtrates wurde im Vakuum entfernt und der Feststoff mittels Säulenchromatografie (Silica, Hexan : Essigsäureethylester 2:1) aufgereinigt. Das Produkt wurde im Vakuum getrocknet und als orangefarbener Feststoff $(24,15 \mathrm{~g}, 94,2 \mathrm{mmol}, 79 \%)$ erhalten. ${ }^{36}$

${ }^{1}$ H-NMR $\left(300 \mathrm{MHz}, \mathrm{CDCl}_{3}\right): \delta(\mathrm{ppm})=7.95-7.86(\mathrm{~m}, 4 \mathrm{H}, 4-\mathrm{C}, 5-\mathrm{C})$, 7.53-7.41 (m, $3 \mathrm{H}, 7-\mathrm{C}, 8-\mathrm{C}), 7.05-7.00(\mathrm{~m}, 2 \mathrm{H}, 6-\mathrm{C}), 4.22(\mathrm{t}, J=6.0 \mathrm{~Hz}$, $2 \mathrm{H}, 3-\mathrm{C}), 3.90(\mathrm{t}, J=6.0 \mathrm{~Hz}, 2 \mathrm{H}, 1-\mathrm{C}), 2.10(\mathrm{p}, J=6.0 \mathrm{~Hz}, 2 \mathrm{H}, 2-\mathrm{C}), 1.59$ (s br, $1 \mathrm{H}, \mathrm{OH}$ ).

ESI-MS $(m / z): 257.1[\mathrm{M}+\mathrm{H}]^{+}, 279.1[\mathrm{M}+\mathrm{Na}]^{+}$. 
Synthese von 6-(4-(Phenyldiazenyl)phenoxy)hexan-1-ol

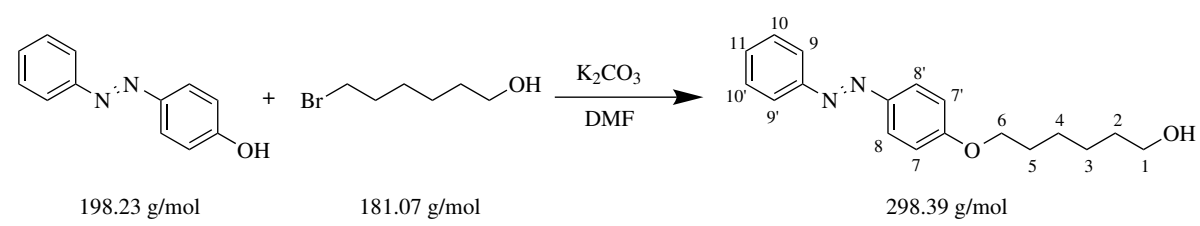

4-Phenylazophenol (4,38 g, 22,1 mmol, 1,00 Äq.), Kaliumcarbonat (4,58 g, 33,1 mmol, 1,50 Äq.) und eine Spatelspitze Kaliumiodid wurden in DMF $(20 \mathrm{ml})$ gelöst und für eine Stunde gerührt. Es wurde 6-Bromhexanol (5,0 g, 27,6 mmol, 1,25 Äq.) langsam zugetropft und das Reaktionsgemisch über Nacht refluxiert. Das Lösungsmittel wurde im Vakuum entfernt. Der Feststoff wurde mit Diethylether aufgenommen und filtriert. Der Rückstand wurde mit Diethylether gewaschen. Das Lösungsmittel des Filtrates wurde im Vakuum entfernt und der Feststoff mittels Säulenchromatografie (Silica, Hexan : Essigsäureethylester 2:1) aufgereinigt. Das Produkt wurde im Vakuum getrocknet und als orangefarbener Feststoff $(5,40 \mathrm{~g}, 18,1 \mathrm{mmol}, 82 \%)$ erhalten.

${ }^{1} \mathbf{H}-\mathrm{NMR}\left(300 \mathrm{MHz}, \mathrm{CD}_{2} \mathrm{Cl}_{2}\right): \delta(\mathrm{ppm})=7.94-7.84(\mathrm{~m}, 4 \mathrm{H}, 7-\mathrm{C}, 8-\mathrm{C})$, 7.56-7.41 (m, 3 H, 10-C, 11-C) 7.06-6.98 (m, $2 \mathrm{H}, 9-\mathrm{C}), 4.06(\mathrm{t}, J=6.5 \mathrm{~Hz}$, $2 \mathrm{H}, 6-\mathrm{C}), 3.62(\mathrm{t}, J=6.5 \mathrm{~Hz}, 2 \mathrm{H}, 1-\mathrm{C}), 1.65-1.37(\mathrm{~m}, 8 \mathrm{H}, 2-\mathrm{C}-5-\mathrm{C})$.

ESI-MS $(m / z): 299.2[\mathrm{M}+\mathrm{H}]^{+}, 321.2[\mathrm{M}+\mathrm{Na}]^{+}$. 
Synthese von 11-(4-(Phenyldiazenyl)phenoxy)undecan-1-ol<smiles>Oc1ccc(NNc2ccccc2)cc1</smiles>

$198.23 \mathrm{~g} / \mathrm{mol}$

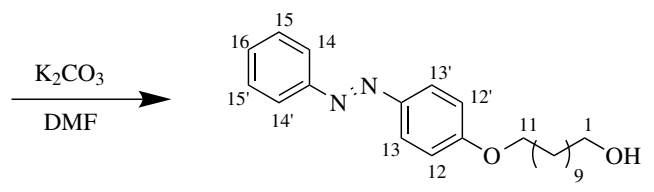

$368.29 \mathrm{~g} / \mathrm{mol}$

4-Phenylazophenol (3,15 g, 15,9 mmol, 1,00 Äq.), Kaliumcarbonat (3,30 g, 23,9 mmol, 1,50 Äq.) und eine Spatelspitze Kaliumiodid wurden in DMF ( $5 \mathrm{ml}$ ) gelöst und für eine Stunde gerührt. Es wurde 11-Bromundecanol (5,0 g, 19,9 mmol, 1,25 Äq.) langsam zugegeben und das Reaktionsgemisch über Nacht refluxiert. Das Lösungsmittel wurde im Vakuum entfernt. Der Feststoff wurde mit Diethylether aufgenommen und filtriert. Der Rückstand wurde mit Diethylether gewaschen. Das Lösungsmittel des Filtrates wurde im Vakuum entfernt und der Feststoff mittels Säulenchromatografie (Silica, Hexan : Essigsäureethylester 2:1) aufgereinigt. Das Produkt wurde im Vakuum getrocknet und als orangefarbener Feststoff $(4,45 \mathrm{~g}, 12,1 \mathrm{mmol}, 76 \%)$ erhalten.

${ }^{1}$ H-NMR $\left(300 \mathrm{MHz}, \mathrm{CDCl}_{3}\right): \delta(\mathrm{ppm})=7.94-7.85(\mathrm{~m}, 4 \mathrm{H}, 12-\mathrm{C}, 13-\mathrm{C})$, 7.56-7.40 (m, 3 H, 15-C, 16-C) 7.04-6.97 (m, 2 H, 14-C), $4.04(\mathrm{t}, J=6.6 \mathrm{~Hz}$, $2 \mathrm{H}, 11-\mathrm{C}), 3.64$ (t, J = 6.6 Hz, $2 \mathrm{H}, 1-\mathrm{C}), 1.41-1.15$ (m, $18 \mathrm{H}, 2-\mathrm{C}-10-\mathrm{C})$.

ESI-MS $(m / z): 369.2[\mathrm{M}+\mathrm{H}]^{+}, 391.2[\mathrm{M}+\mathrm{Na}]^{+}$. 
Synthese von 4-(Phenyldiazenyl)phenoxymethacrylat<smiles>C=C(C)C(=O)Cl</smiles>

Unter trockenen Inertgasbedingungen wurde 4-(Phenyldiazenyl)phenol (5,00 g, 25,2 mmol, 1,00 Äq.) vorgelegt und in THF (40 ml) gelöst. Bei $0{ }^{\circ} \mathrm{C}$ wurde Triethylamin $(3,85 \mathrm{ml}, 27,7 \mathrm{mmol}, 1,10$ Äq.) zugetropft. Anschließend wurde langsam Methacryloylchlorid $(3,66 \mathrm{ml}$, 37,8 mmol, 1,50 Äq.) zugetropft und für 20 Stunden gerührt. Zum Reaktionsgemisch wurde Wasser gegeben und das THF im Vakuum entfernt. Es wurde mit Diethylether extrahiert. Die organische Phase wurde mit 1 M Salzsäure, ges. Natriumhydrogencarbonat-Lösung und Wasser gewaschen. Nach Trocknen über Magnesiumsulfat wurde das Lösungsmittel im Vakuum entfernt. Säulenchromatografische Aufreinigung (Silica, Pentan : Essigsäureethylester, 2:1) lieferte das Produkt als orangefarbenen Feststoff (5,4 g, 20,4 mmol, 81 \%).

${ }^{1}$ H-NMR $\left(300 \mathrm{MHz}, \mathrm{CD}_{2} \mathrm{Cl}_{2}\right): \delta(\mathrm{ppm})=7.98(\mathrm{dt}, J=9.1 \mathrm{~Hz}, 2.1 \mathrm{~Hz}$, $2 \mathrm{H}, 4-\mathrm{C}), 7.92(\mathrm{~m}, 2 \mathrm{H}, 5-\mathrm{C}), 7.51(\mathrm{~m}, 3 \mathrm{H}, 6-\mathrm{C}, 7-\mathrm{C}), 7.29(\mathrm{dt}, J=9.1 \mathrm{~Hz}$, $2.1 \mathrm{~Hz}, 2 \mathrm{H}, 3-\mathrm{C}), 6.30\left(\mathrm{dq}, J=1.5 \mathrm{~Hz}, 0.9 \mathrm{~Hz}, 1 \mathrm{H}, \mathrm{H}_{\text {trans }}\right), 5.80$ (dq, $J=$ $1.5 \mathrm{~Hz}, 1.5 \mathrm{~Hz}, 1 \mathrm{H}, \mathrm{H}_{\mathrm{cis}}$ ), 2.09 (dd, $J=1.5 \mathrm{~Hz}, 0.9 \mathrm{~Hz}, 3 \mathrm{H}, 2-\mathrm{C}$ ).

ESI-MS $(m / z): 267.2[\mathrm{M}+\mathrm{H}]^{+}, 289.2[\mathrm{M}+\mathrm{Na}]^{+}$. 
Synthese von 3-(4-(Phenyldiazenyl)phenoxy)propylmethacrylat

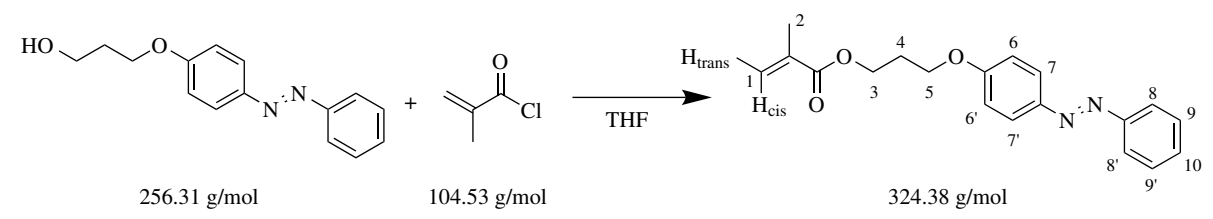

Unter trockenen Inertgasbedingungen wurde 3-(4-(Phenyldiazenyl)phenoxy)propan-1-ol (24,15 g, 94,2 mmol, 1,00 Äq.) vorgelegt und in THF $(150 \mathrm{ml})$ gelöst. Bei $0{ }^{\circ} \mathrm{C}$ wurde Triethylamin $(12,7 \mathrm{ml}$, 103,6 mmol, 1,10 Äq.) zugetropft. Anschließend wurde langsam Methacryloylchlorid (13,68 ml, 141,3 mmol, 1,50 Äq.) zugetropft und für 20 Stunden gerührt. Zum Reaktionsgemisch wurde Wasser gegeben und das THF im Vakuum entfernt. Es wurde mit Diethylether extrahiert. Die organische Phase wurde mit 1 M Salzsäure, ges. Natriumhydrogencarbonat-Lösung und Wasser gewaschen. Nach Trocknen über Magnesiumsulfat wurde das Lösungsmittel im Vakuum entfernt. Säulenchromatografische Aufreinigung (Silica, Pentan : Essigsäureethylester, 2:1) lieferte das Produkt als orangefarbenen Feststoff $(21,9 \mathrm{~g}, 67,6 \mathrm{mmol}, 72 \%){ }^{36}$

${ }^{1} \mathrm{H}-\mathrm{NMR}\left(300 \mathrm{MHz}, \mathrm{CD}_{2} \mathrm{Cl}_{2}\right): \delta(\mathrm{ppm})=7.94-7.85(\mathrm{~m}, 4 \mathrm{H}, 6 \mathrm{C}, 7 \mathrm{C})$, 7.55-7.42 (m, $3 \mathrm{H}, 9 \mathrm{C}, 10 \mathrm{C}), 7.06-7.01(\mathrm{~m}, 2 \mathrm{H}, 8 \mathrm{C}), 6.10(\mathrm{dq}, J=1.6 \mathrm{~Hz}$, $\left.1.0 \mathrm{~Hz}, 1 \mathrm{H}, \mathrm{H}_{\mathrm{cis}}\right), 5.57\left(\mathrm{qd}, J=1.6 \mathrm{~Hz}, 1.6 \mathrm{~Hz}, 1 \mathrm{H}, \mathrm{H}_{\text {trans }}\right), 4.35(\mathrm{t}, J=$ $6.2 \mathrm{~Hz}, 2 \mathrm{H}, 5 \mathrm{C}), 4.17(\mathrm{t}, J=6.2 \mathrm{~Hz}, 2 \mathrm{H}, 3 \mathrm{C}), 2.21(\mathrm{p}, J=6.2 \mathrm{~Hz}, 2 \mathrm{H}, 4 \mathrm{C})$, $1.94(\mathrm{dd}, J=1.6 \mathrm{~Hz}, 1.0 \mathrm{~Hz}, 3 \mathrm{H}, 2 \mathrm{C})$.

ESI-MS $(m / z): 325.2[\mathrm{M}+\mathrm{H}]^{+}, 347.1[\mathrm{M}+\mathrm{Na}]^{+}$. 
Synthese von 6-(4-(Phenyldiazenyl)phenoxy)hexylmethacrylat

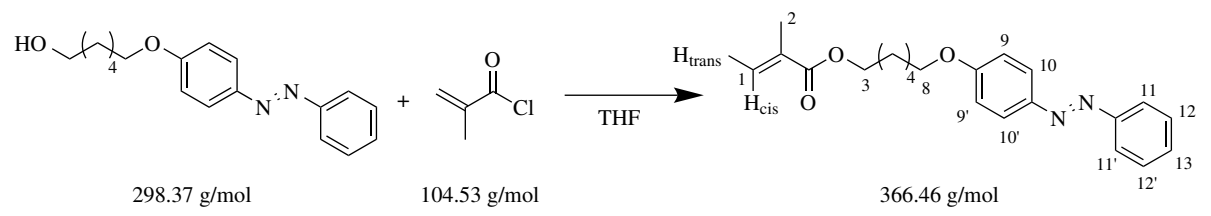

Unter trockenen Inertgasbedingungen wurde 6-(4-(Phenyldiazenyl)phenoxy)hexan-1-ol (4,44 g, 14,9 mmol, 1,00 Äq.) vorgelegt und in THF (40 ml) gelöst. Bei $0{ }^{\circ} \mathrm{C}$ wurde Triethylamin $(2,5 \mathrm{ml}, 16,4 \mathrm{mmol}$, 1,10 Äq.) zugetropft. Anschließend wurde langsam Methacryloylchlorid (3,50 ml, 29,8 mmol, 2,00 Äq.) zugetropft und für 20 Stunden gerührt. Zum Reaktionsgemisch wurde Wasser gegeben und das THF im Vakuum entfernt. Es wurde mit Diethylether extrahiert. Die organische Phase wurde mit 1 M Salzsäure, ges. NatriumhydrogencarbonatLösung und Wasser gewaschen. Nach Trocknen über Magnesiumsulfat wurde das Lösungsmittel im Vakuum entfernt. Säulenchromatografische Aufreinigung (Silica, Hexan : Essigsäureethylester, 2:1) lieferte das Produkt als orangefarbenen Feststoff $(4,15 \mathrm{~g}, 11,3 \mathrm{mmol}, 76 \%)$.

${ }^{1}$ H-NMR (300 MHz, $\left.\mathrm{CD}_{2} \mathrm{Cl}_{2}\right): \delta(\mathrm{ppm})=7.94-7.84(\mathrm{~m}, 4 \mathrm{H}, 9-\mathrm{C}, 10-\mathrm{C})$, 7.55-7.42 (m, $3 \mathrm{H}, 12-\mathrm{C}, 13-\mathrm{C}), 7.02(\mathrm{~d}, J=9.1 \mathrm{~Hz}, 2 \mathrm{H}, 11-\mathrm{C}), 6.07$ (dq, $\left.J=2.0 \mathrm{~Hz}, 1.0 \mathrm{~Hz}, 1 \mathrm{H}, \mathrm{H}_{\mathrm{cis}}\right), 5.55\left(\mathrm{dq}, J=1.6 \mathrm{~Hz}, 1.6 \mathrm{~Hz}, 1 \mathrm{H}, \mathrm{H}_{\text {trans }}\right), 4.15$ $(\mathrm{t}, J=6.6 \mathrm{~Hz}, 2 \mathrm{H}, 8-\mathrm{C}) 4.06(\mathrm{t}, J=6.6 \mathrm{~Hz}, 2 \mathrm{H}, 3-\mathrm{C}) 1.94(\mathrm{~m}, 3 \mathrm{H}, 2-\mathrm{C})$, 1.59-1.41 (m, 8 H, 4-C-7-C).

ESI-MS $(m / z): 367.2[\mathrm{M}+\mathrm{H}]^{+}, 389.2[\mathrm{M}+\mathrm{Na}]^{+}$. 
Synthese von 11-(4-(Phenyldiazenyl)phenoxy)undecylmethacrylat

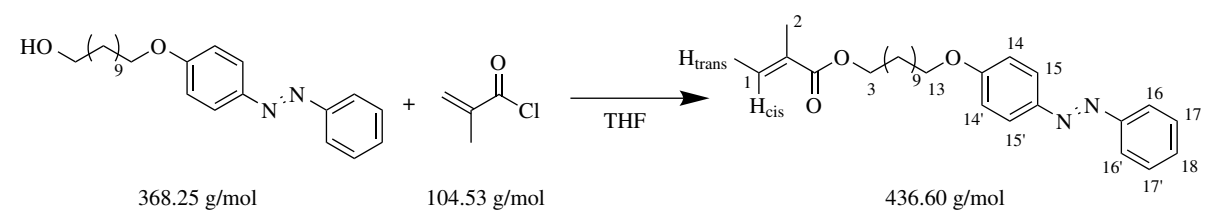

Unter trockenen Inertgasbedingungen wurde 11-(4-(Phenyldiazenyl)phenoxy)undecan-1-ol (1,55 g, 4,21 mmol, 1,00 Äq.) vorgelegt und in THF $(40 \mathrm{ml})$ gelöst. Bei $0{ }^{\circ} \mathrm{C}$ wurde Triethylamin $(0,7 \mathrm{ml}, 4,63 \mathrm{mmol}$, 1,10 Äq.) zugetropft. Anschließend wurde langsam Methacryloylchlorid (1,00 ml, 8,42 mmol, 2,00 Äq.) zugetropft und für 20 Stunden gerührt. Zum Reaktionsgemisch wurde Wasser gegeben und das THF im Vakuum entfernt. Es wurde mit Diethylether extrahiert. Die organische Phase wurde mit 1 M Salzsäure, ges. NatriumhydrogencarbonatLösung und Wasser gewaschen. Nach Trocknen über Magnesiumsulfat wurde das Lösungsmittel im Vakuum entfernt. Säulenchromatografische Aufreinigung (Silica, Hexan : Essigsäureethylester, 2:1) lieferte das Produkt als orangefarbenen Feststoff (1,53 g, 3,49 mmol, $83 \%)$.

${ }^{1}$ H-NMR $\left(300 \mathrm{MHz}, \mathrm{CDCl}_{3}\right): \delta(\mathrm{ppm})=7.89(\mathrm{t}, J=9.5 \mathrm{~Hz}, 4 \mathrm{H}, 14-\mathrm{C}$, 15-C), 7.54-7.41 (m, 3 H, 17-C, 18-C), 7.00 (d, J = 7.7 Hz, 2 H 16-C), 6.09 $\left(\mathrm{s}, 1 \mathrm{H}, \mathrm{H}_{\mathrm{cis}}\right), 5.54\left(\mathrm{~s}, 1 \mathrm{H}, \mathrm{H}_{\text {trans }}\right), 4.13(\mathrm{t}, J=6.4 \mathrm{~Hz}, 2 \mathrm{H}, 13-\mathrm{C}), 4.04(\mathrm{t}, J$ $=6.4 \mathrm{~Hz}, 2 \mathrm{H}, 3-\mathrm{C}), 1.92$ (s, 3 H, 2-C), 1.43-1.17 (m, 18 H, 4-C-12-C).

ESI-MS $(m / z): 437.3[\mathrm{M}+\mathrm{H}]^{+}, 459.3[\mathrm{M}+\mathrm{Na}]^{+}$. 


\subsection{Synthesen der photoschaltbaren Polymere für die Untersuchung der Kinetik}

\section{Synthese von Poly-4-(Phenyldiazenyl)phenoxymethacrylat}

4-(Phenyldiazenyl)phenoxymethacrylat (1,0 g, 3,76 mmol, 300 Äq.), 4Cyano-4-[(dodecylsulfanylthiocarbonyl)sulfanyl]-pentansäure in $\mathrm{N}, \mathrm{N}$ Dimethylformamid (125 $\mu \mathrm{l}, 0,1 \mathrm{~mol} / 1,12,5 \mu \mathrm{mol}, 1,0$ Äq.) und 1,1'Azobis(cyclohexancarbonitril) in N,N-Dimethylformamid (25,0 $\mu \mathrm{l}, 0,1$

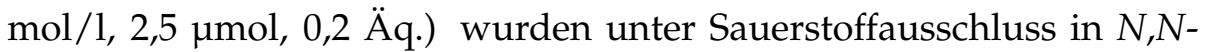
Dimethylformamid $(3,0 \mathrm{ml})$ gelöst und für $19 \mathrm{~h}$ bei $95^{\circ} \mathrm{C}$ gerührt. Die Reaktionsmischung wurde anschließend in Methanol $(45 \mathrm{ml})$ gegossen und für mindestens 30 min bei 9000 Umdrehungen pro Minute zentrifugiert. Die überstehende Lösung wurde dekantiert und der Feststoff in Tetrahydrofuran $(5 \mathrm{ml})$ aufgenommen. Der Fällungs-ZentrifugationsAufnahme-Zyklus wurde 3 mal durchgeführt. Das Produkt wurde anschließend im Vakuum getrocknet.

Synthese von Poly-3-(4-(Phenyldiazenyl)phenoxy)propylmethacrylat

3-(4-(Phenyldiazenyl)phenoxy)propylmethacrylat $(4,0 \mathrm{~g}, 12,3 \mathrm{mmol}$, 300 Äq.), 4-Cyano-4-[(dodecylsulfanylthiocarbonyl)sulfanyl]-pentansäure in N,N-Dimethylformamid (412 $\mu \mathrm{l}, 0,1 \mathrm{~mol} / \mathrm{l}, 41,2 \mu \mathrm{mol}, 1,0$ Äq.) und 1,1'-Azobis(cyclohexancarbonitril) in $N, N$-Dimethylformamid (82,0 $\mu \mathrm{l}, 0,1 \mathrm{~mol} / \mathrm{l}, 8,2 \mu \mathrm{mol}, 0,2$ Äq.) wurden unter Sauerstoffausschluss in N,N-Dimethylformamid $(15,0 \mathrm{ml})$ gelöst und für $24 \mathrm{~h}$ bei $95{ }^{\circ} \mathrm{C}$ gerührt. Die Reaktionsmischung wurde anschließend in Methanol (45 ml) gegossen und für mindestens $30 \mathrm{~min}$ bei 9000 Umdrehungen pro Minute zentrifugiert. Die überstehende Lösung wurde dekantiert und der Feststoff in Tetrahydrofuran $(5 \mathrm{ml})$ aufgenommen. Der Fällungs-Zentrifugations-Aufnahme-Zyklus wurde 3 mal durchgeführt. Das Produkt wurde anschließend im Vakuum getrocknet. 


\section{Synthese von Poly-6-(4-(Phenyldiazenyl)phenoxy)hexylmethacrylat}

6-(4-(Phenyldiazenyl)phenoxy)hexylmethacrylat $(1,0 \mathrm{~g}, 2,73 \mathrm{mmol}$, 300 Äq.), 4-Cyano-4-[(dodecylsulfanylthiocarbonyl)sulfanyl]-pentansäure in $N, N$-Dimethylformamid (90,1 $\mu \mathrm{l}, 0,1 \mathrm{~mol} / 1,9,1 \mu \mathrm{mol}, 1,0$ Äq.) und 1,1'-Azobis(cyclohexancarbonitril) in $N, N$-Dimethylformamid $(18,0 \mu \mathrm{l}, 0,1 \mathrm{~mol} / 1,1,8 \mu \mathrm{mol}, 0,2$ Äq.) wurden unter Sauerstoffausschluss in N,N-Dimethylformamid $(3,0 \mathrm{ml})$ gelöst und für $19 \mathrm{~h}$ bei $95{ }^{\circ} \mathrm{C}$ gerührt. Die Reaktionsmischung wurde anschließend in Methanol (45 ml) gegossen und für mindestens $30 \mathrm{~min}$ bei 8000 Umdrehungen pro Minute zentrifugiert. Die überstehende Lösung wurde dekantiert und der Feststoff in Tetrahydrofuran $(5 \mathrm{ml})$ aufgenommen. Der Fällungs-Zentrifugations-Aufnahme-Zyklus wurde 3 mal durchgeführt. Das Produkt wurde anschließend im Vakuum getrocknet.

\section{Synthese von}

Poly-11-(4-(Phenyldiazenyl)phenoxy)undecylmethacrylat

11-(4-(Phenyldiazenyl)phenoxy)undecylmethacrylat (525 mg, 1,20 mmol, 300 Äq.), 4-Cyano-4-[(dodecylsulfanylthiocarbonyl)sulfanyl]-pentansäure in N,N-Dimethylformamid $(40,0 \mu \mathrm{l}, 0,1 \mathrm{~mol} / \mathrm{l}$, 4,0 $\mu \mathrm{mol}, 1,0$ Äq.) und 1,1'-Azobis(cyclohexancarbonitril) in $N, N$ Dimethylformamid $(8,0 \mu \mathrm{l}, 0,1 \mathrm{~mol} / 1,0,8 \mu \mathrm{mol}, 0,2$ Äq.) wurden unter Sauerstoffausschluss in N,N-Dimethylformamid (1,5 ml) gelöst und für $19 \mathrm{~h}$ bei $95{ }^{\circ} \mathrm{C}$ gerührt. Die Reaktionsmischung wurde anschließend in Methanol (45 ml) gegossen und für mindestens $30 \mathrm{~min}$ bei 6000 Umdrehungen pro Minute zentrifugiert. Die überstehende Lösung wurde dekantiert und der Feststoff in Tetrahydrofuran $(5 \mathrm{ml})$ aufgenommen. Der Fällungs-Zentrifugations-Aufnahme-Zyklus wurde 3 mal durchgeführt. Das Produkt wurde anschließend im Vakuum getrocknet. 


\subsection{Synthesen der photoschaltbaren Blockcopolymere}

\section{Synthese von Poly-3-(4-(Phenyldiazenyl)phenoxy)propylmethacrylat}

3-(4-(Phenyldiazenyl)phenoxy)propylmethacrylat (6,5 g, 20,0 mmol, 300 Äq.), 4-Cyano-4-[(dodecylsulfanylthiocarbonyl)sulfanyl]pentansäure (27,0 mg, 67,0 $\mu \mathrm{mol}, 1,0$ Äq.) und 1,1'-Azobis(cyclohexancarbonitril) in N,N-Dimethylformamid (130 $\mu \mathrm{l}, 0,1 \mathrm{~mol} / \mathrm{l}$, 13,0 pmol, 0,2 Äq.) wurden unter Sauerstoffausschluss in $N, N$ Dimethylformamid $(15,0 \mathrm{ml})$ gelöst und für $24 \mathrm{~h}$ bei $90{ }^{\circ} \mathrm{C}$ gerührt. Die Reaktionsmischung wurde anschließend in Methanol (45 ml) gegossen und für mindestens $30 \mathrm{~min}$ bei 9000 Umdrehungen pro Minute zentrifugiert. Die überstehende Lösung wurde dekantiert und der Feststoff in Tetrahydrofuran (5 ml) aufgenommen. Der FällungsZentrifugations-Aufnahme-Zyklus wurde 3 mal durchgeführt. Das Produkt wurde anschließend im Vakuum getrocknet.

Synthese von Poly-3-(4-(Phenyldiazenyl)phenoxy)propylmethacrylatblock-poly-butylacrylat I (BCP I)

BCP I wurde vom Autor der Arbeit bereits bei der Erstellung von [36] hergestellt und unverändert in dieser Arbeit genutzt. Die zur Vollständigkeit aus [36] übernommene Synthesevorschrift lautet:

Poly-3-(4-(Phenyldiazenyl)phenoxy)propylmethacrylat (104,8 $\mathrm{mg}$, 6,48 $\mathrm{mmol}, 1,0$ Äq.) und 2,2'-Azobis(2-methylpropionitril) in $N, N$ Dimethylformamid (13,0 $\mu \mathrm{l}, 0,1 \mathrm{~mol} / \mathrm{l}, 1,3 \mu \mathrm{mol}, 0,2$ Äq.) wurden vorgelegt und unter Sauerstoffausschluss 1,2-Dimethoxyethan $(0,3 \mathrm{ml})$ und n-Butylacrylat (332,5 mg, 2,60 mmol, 400 Äq.) zugegeben. Die Reaktionsmischung wurde für $72 \mathrm{~h}$ auf $65{ }^{\circ} \mathrm{C}$ erhitzt. Anschließend wurden flüchtige Komponenten im Vakuum entfernt und das Produkt erhalten. 
Synthese von Poly-3-(4-(Phenyldiazenyl)phenoxy)propylmethacrylatblock-poly-butylacrylat II (BCP II)

Poly-3-(4-(Phenyldiazenyl)phenoxy)propylmethacrylat (100 mg, 5,88 $\mathrm{mol}, 1,0$ Äq.) und 2,2'-Azobis(2-methylpropionitril) in $\mathrm{N}, \mathrm{N}$ Dimethylformamid (19,6 $\mu \mathrm{l}, 0,1 \mathrm{~mol} / \mathrm{l}, 1,96 \mu \mathrm{mol}, 0,33$ Äq.) wurden vorgelegt und unter Sauerstoffausschluss 1,2-Dimethoxyethan $(0,3 \mathrm{ml})$ und n-Butylacrylat (264 mg, 2,06 mmol, 350 Äq.) zugegeben. Die Reaktionsmischung wurde für $18 \mathrm{~h}$ auf $70{ }^{\circ} \mathrm{C}$ erhitzt. Anschließend wurden flüchtige Komponenten im Vakuum entfernt und das Produkt erhalten.

Synthese von Poly-3-(4-(Phenyldiazenyl)phenoxy)propylmethacrylatblock-poly-butylacrylat III (BCP III)

Poly-3-(4-(Phenyldiazenyl)phenoxy)propylmethacrylat (100 mg, 5,88 $\mathrm{mol}, 1,0$ Äq.) und 2,2'-Azobis(2-methylpropionitril) in $\mathrm{N}, \mathrm{N}$ Dimethylformamid (19,6 $\mu \mathrm{l}, 0,1 \mathrm{~mol} / \mathrm{l}, 1,96 \mu \mathrm{mol}, 0,33$ Äq.) wurden vorgelegt und unter Sauerstoffausschluss 1,2-Dimethoxyethan $(0,3 \mathrm{ml})$ und n-Butylacrylat (301 mg, 2,35 mmol, 400 Äq.) zugegeben. Die Reaktionsmischung wurde für $18 \mathrm{~h}$ auf $70{ }^{\circ} \mathrm{C}$ erhitzt. Anschließend wurden flüchtige Komponenten im Vakuum entfernt und das Produkt erhalten.

Synthese von Poly-3-(4-(Phenyldiazenyl)phenoxy)propylmethacrylatblock-poly-butylacrylat IV (BCP IV)

Poly-3-(4-(Phenyldiazenyl)phenoxy)propylmethacrylat (100 mg, 5,88 $\mathrm{mol}, 1,0$ Äq.) und 2,2'-Azobis(2-methylpropionitril) in $\mathrm{N}, \mathrm{N}$ Dimethylformamid (19,6 $\mu \mathrm{l}, 0,1 \mathrm{~mol} / \mathrm{l}, 1,96 \mu \mathrm{mol}, 0,33$ Äq.) wurden vorgelegt und unter Sauerstoffausschluss 1,2-Dimethoxyethan $(0,3 \mathrm{ml})$ und n-Butylacrylat (377 mg, 2,94 mmol, 500 Äq.) zugegeben. Die Reaktionsmischung wurde für $18 \mathrm{~h}$ auf $70{ }^{\circ} \mathrm{C}$ erhitzt. Anschließend wurden flüchtige Komponenten im Vakuum entfernt und das Produkt erhalten. 


\subsection{Synthese von Homopolymeren zur Herstellung von Blockcopolymer-Homopolymer-Kompositen}

\section{Synthese von Poly-3-(4-(Phenyldiazenyl)phenoxy)propylmethacrylat}

3-(4-(Phenyldiazenyl)phenoxy)propylmethacrylat (6,5 g, 20,0 mmol, 300 Äq.), 4-Cyano-4-[(dodecylsulfanylthiocarbonyl)sulfanyl]pentansäure (27,0 mg, 67,0 $\mu \mathrm{mol}, 1,0$ Äq.) und 1,1'-Azobis(cyclohexancarbonitril) in N,N-Dimethylformamid (130 $\mu \mathrm{l}, 0,1 \mathrm{~mol} / \mathrm{l}$, 13,0 pmol, 0,2 Äq.) wurden unter Sauerstoffausschluss in $N, N$ Dimethylformamid $(15,0 \mathrm{ml})$ gelöst und für $24 \mathrm{~h}$ bei $90{ }^{\circ} \mathrm{C}$ gerührt. Die Reaktionsmischung wurde anschließend in Methanol $(45 \mathrm{ml})$ gegossen und für mindestens $30 \mathrm{~min}$ bei 9000 Umdrehungen pro Minute zentrifugiert. Die überstehende Lösung wurde dekantiert und der Feststoff in Tetrahydrofuran (5 ml) aufgenommen. Der FällungsZentrifugations-Aufnahme-Zyklus wurde 3 mal durchgeführt. Das Produkt wurde anschließend im Vakuum getrocknet.

\section{Synthese von Polybutylacrylat}

n-Butylacrylat (5,0 g, 39 mmol, 300 Äq.), 4-Cyano-4-[(dodecylsulfanylthiocarbonyl)sulfanyl]-pentansäure (52,5 mg, 13,0 $\mu \mathrm{mol}, 1,0$ Äq.) und 2,2'-Azobis(2-methylpropionitril) in $\mathrm{N}, \mathrm{N}$-Dimethylformamid (650 $\mu \mathrm{l}$, 0,1 mol/l, 65 umol, 0,2 Äq.) wurden in Toluol (10 ml) gelöst. Die Reaktionsmischung wurde 10 Minuten mit Argon gespült und im Anschluss für $24 \mathrm{~h}$ bei $60{ }^{\circ} \mathrm{C}$ gerührt. Anschließend wurden flüchtige Komponenten im Vakuum entfernt und das Produkt erhalten.

\section{Herstellung der Blockcopolymer-Homopolymer-Komposite}

Polybutylacrylat in THF (1,0 Gew.-\%), Poly-3-(4-(Phenyldiazenyl)phenoxy)propylmethacrylat in THF $(1,0$ Gew.- $\%)$ und BCP I in THF $(2,5$ Gew.- $\%)$ wurden in den angegebenen Verhältnissen gemischt. Das Lösungsmittel wurde anschließend im Vakuum entfernt. 
Experimentalteil

\begin{tabular}{lccc}
\hline Ansatz & BCP I in THF & PBA in THF & PAzoPMA in THF \\
\hline BCP I+10 & $800 \mathrm{mg}$ & $129,2 \mathrm{mg}$ & $70,8 \mathrm{mg}$ \\
BCP I+20 & $800 \mathrm{mg}$ & $258,4 \mathrm{mg}$ & $141,6 \mathrm{mg}$ \\
BCP I+30 & $800 \mathrm{mg}$ & $387,6 \mathrm{mg}$ & $212,4 \mathrm{mg}$ \\
BCP I+40 & $800 \mathrm{mg}$ & $516,8 \mathrm{mg}$ & $283,2 \mathrm{mg}$ \\
BCP I+50 & $800 \mathrm{mg}$ & $646,0 \mathrm{mg}$ & $354,0 \mathrm{mg}$ \\
\hline
\end{tabular}

\subsection{Herstellung des Blockcopolymers zur Herstellung von photospaltbaren, befüllten Polymeraggregaten}

Synthese von Poly-3-(4-(Phenyldiazenyl)phenoxy)propylmethacrylatblock-poly-(2-(Dimethylamino)ethylmethacrylat) (BCP V)

BCP V wurde vom Autor der Arbeit bei der Erstellung von [36] hergestellt und unverändert in dieser Arbeit genutzt. Die Synthesevorschrift lautet:

3-(4-(Phenyldiazenyl)phenoxy)propylmethacrylat $(2,0 \mathrm{~g}, 6,1 \mathrm{mmol}$, 300 Äq.), 4-Cyano-4-[(dodecylsulfanylthiocarbonyl)sulfanyl]-pentansäure in N,N-Dimethylformamid (205 $\mu \mathrm{l}, 0,1 \mathrm{~mol} / \mathrm{l}, 20,5 \mu \mathrm{mol}, 1,0$ Äq.) und 1,1'-Azobis(cyclohexancarbonitril) in $N, N$-Dimethylformamid (31,0 $\mu \mathrm{l}, 0,1 \mathrm{~mol} / \mathrm{l}, 3,1 \mu \mathrm{mol}, 0,15$ Äq.) wurden unter Sauerstoffausschluss in $\mathrm{N}, \mathrm{N}$-Dimethylformamid $(7,0 \mathrm{ml})$ gelöst und für $24 \mathrm{~h}$ bei $90{ }^{\circ} \mathrm{C}$ gerührt. Die Reaktionsmischung wurde anschließend in Methanol (45 ml) gegossen und für mindestens $30 \mathrm{~min}$ bei $9000 \mathrm{Um}$ drehungen pro Minute zentrifugiert. Die überstehende Lösung wurde dekantiert und der Feststoff in Tetrahydrofuran $(5 \mathrm{ml})$ aufgenommen. Der Fällungs-Zentrifugations-Aufnahme-Zyklus wurde 3 mal durchgeführt. Das Zwischenprodukt wurde anschließend im Vakuum getrocknet.

Das Zwischenprodukt (360 mg, $18600 \mathrm{~g} / \mathrm{mol}, 19,3$ mmol, 1,00 Äq.), 2(Dimethylamino)ethylmethacrylat (608,4 mg, 3,87 mmol, 200 Äq.) und 2,2'-Azobis(2-methylpropionitril) in DMF (38,8 $\mu \mathrm{l}, 0,1 \mathrm{~mol} / 1,3,88 \mu \mathrm{mol}$, 0,2 Äq.) wurden unter Inertgasatmosphäre in 1,2-Dimethoxyethan 
$(1,0 \mathrm{ml})$ gelöst und bei $60^{\circ} \mathrm{C}$ für $44 \mathrm{~h}$ gerührt. Flüchtige Komponenten wurden bei $60^{\circ} \mathrm{C}$ unter vermindertem Druck entfernt und das Produkt erhalten.

\subsection{Herstellung der Polymere für die Wärmeleitfähigkeitsmessungen}

\section{Synthese von Poly-3-(4-(Phenyldiazenyl)phenoxy)propylmethacrylat}

3-(4-(Phenyldiazenyl)phenoxy)propylmethacrylat (6,5 g, 20,0 mmol, 300 Äq.), 4-Cyano-4-[(dodecylsulfanylthiocarbonyl)sulfanyl]pentansäure $(27,0 \mathrm{mg}, 67,0 \mu \mathrm{mol}, 1,0$ Äq.) und 1,1'-Azobis(cyclohexancarbonitril) (3,6 mg, 16,0 $\mu \mathrm{mol}, 0,25$ Äq.) wurden unter Sauerstoffausschluss in $N, N$-Dimethylformamid $(12,0 \mathrm{ml})$ gelöst und für $17 \mathrm{~h}$ bei $90^{\circ} \mathrm{C}$ gerührt. Die Reaktionsmischung wurde anschließend in Methanol $(45 \mathrm{ml})$ gegossen und für mindestens $30 \mathrm{~min}$ bei 9000 Umdrehungen pro Minute zentrifugiert. Die überstehende Lösung wurde dekantiert und der Feststoff in Tetrahydrofuran $(5 \mathrm{ml})$ aufgenommen. Der Fällungs-Zentrifugations-Aufnahme-Zyklus wurde 3 mal durchgeführt. Das Produkt wurde anschließend im Vakuum getrocknet.

\section{Synthese von Polymethylmethacrylat PMMA I-XI}

Methylmethacrylat (5,0 ml, $48 \mathrm{mmol})$ wurde mit 4-Cyano-4-[(dodecylsulfanylthiocarbonyl)sulfanyl]-pentansäure und 2,2'-Azobis(2methylpropionitril) $(0,1 \mathrm{~mol} / \mathrm{L})$ in Toluol und Toluol $(5 \mathrm{ml})$ gemischt. Die Mischung wurde für 10 Minuten bei $0{ }^{\circ} \mathrm{C}$ mit einem Argonstrom gespült. Anschließend wurde die Mischung für $20 \mathrm{~h}$ bei $60^{\circ} \mathrm{C}$ gerührt. Die Reaktionsmischung wurde anschließend in Methanol (45 ml) gegossen und für mindestens $30 \mathrm{~min}$ bei 9000 Umdrehungen pro Minute zentrifugiert. Die überstehende Lösung wurde dekantiert und der Feststoff in Tetrahydrofuran (5 ml) aufgenommen. Der FällungsZentrifugations-Aufnahme-Zyklus wurde 3 mal durchgeführt. Das Produkt wurde anschließend im Vakuum getrocknet. 
Experimentalteil

\begin{tabular}{lccc}
\hline Ansatz & $\mathrm{m}$ (CDSPA) / mg & $\mathrm{V}(\mathrm{AIBN}) / \mu \mathrm{l}$ & Monomer:RAFT:Initiator \\
\hline PMMA I & 775,0 & 1920 & $25: 1: 0,1$ \\
PMMA II & 387,5 & 960 & $50: 1: 0,1$ \\
PMMA III & 258,5 & 640 & $75: 1: 0,1$ \\
PMMA IV & 189,5 & 470 & $100: 1: 0,1$ \\
PMMA V & 94,9 & 235 & $200: 1: 0,1$ \\
PMMA VI & 31,6 & 78,0 & $600: 1: 0,1$ \\
PMMA VII & 18,9 & 47,0 & $1000: 1: 0,1$ \\
PMMA VIII & 5,53 & 13,7 & $3500: 1: 0,1$ \\
PMMA IX & 2,42 & 6,0 & $6500: 1: 0,1$ \\
\hline PMMA XI & 47,3 & 117,4 & $400: 1: 0,1$ \\
\hline
\end{tabular}

PMMA X wurde bei Sigma-Aldrich erworben und unverändert eingesetzt.

\subsection{Funktionalisierung der AuNP mit PMMA XI}

$\mathrm{Zu}$ einer Lösung der Citrat-stabilisierten Gold-Nanopartikel in Wasser ( $3 \mathrm{mg} / \mathrm{ml}$ ) wurde das dreifache Volumen PMMA in THF $(1 \mathrm{mg} / \mathrm{ml})$ langsam zugetropft und die Mischung für $18 \mathrm{~h}$ gerührt. Die Partikel wurden durch Zentrifugation (12000 rpm, $45 \mathrm{~min}$ ) abgetrennt und die überstehende Lösung verworfen. Die Partikel wurden in THF redispergiert und erneut zentrifugiert. Dieser Vorgang wurde dreifach durchgeführt.

\subsection{Synthese von BCP VI-IX}

\section{Synthese von BCP VI}

Methylmethacrylat (5,0 ml, $48 \mathrm{mmol}, 200$ Äq.) wurde mit 4-Cyano-4-[(dodecylsulfanylthiocarbonyl)sulfanyl]-pentansäure $\quad(94,9 \mathrm{mg}$, 0,235 mmol, 1,00 Äq. und 2,2'-Azobis(2-methylpropionitril) (235 $\mu \mathrm{l}$, 0,1 mol/L, 23,5 $\mathrm{mol}, 0,1$ Äq.) in Toluol und Toluol (5 ml) gemischt. Die Mischung wurde für 10 Minuten bei $0{ }^{\circ} \mathrm{C}$ mit einem Argonstrom gespült. Anschließend wurde die Mischung für $20 \mathrm{~h}$ bei $60^{\circ} \mathrm{C}$ gerührt. 
Die Reaktionsmischung wurde danach in Methanol $(45 \mathrm{ml})$ gegossen und für mindestens 30 min bei 9000 Umdrehungen pro Minute zentrifugiert. Die überstehende Lösung wurde dekantiert und der Feststoff in Tetrahydrofuran $(5 \mathrm{ml})$ aufgenommen. Der Fällungs-ZentrifugationsAufnahme-Zyklus wurde 3 mal durchgeführt. Der farblose Feststoff wurde anschließend im Vakuum getrocknet. Das Zwischenprodukt (500 mg, 29,1 $\mu \mathrm{mol}, 1,0$ Äq.) und 2,2'-Azobis(2-methylpropionitril) in $N, N$-Dimethylformamid $(43,6 \mu \mathrm{l}, 0,1 \mathrm{~mol} / \mathrm{l}, 4,36 \mu \mathrm{mol}, 0,15$ Äq.) wurden vorgelegt und unter Sauerstoffausschluss 1,2-Dimethoxyethan $(0,3 \mathrm{ml})$ und n-Butylacrylat (730 mg, 5,7 mmol, 195 Äq.) zugegeben. Die Reaktionsmischung wurde für $24 \mathrm{~h}$ auf $60^{\circ} \mathrm{C}$ erhitzt. Anschließend wurden flüchtige Komponenten im Vakuum entfernt und das Produkt erhalten.

\section{Synthese von BCP VII}

Methylmethacrylat (5,0 ml, $48 \mathrm{mmol}, 600$ Äq.) wurde mit 4-Cyano-4-[(dodecylsulfanylthiocarbonyl)sulfanyl]-pentansäure $\quad(31,6 \mathrm{mg}$, 78,0 $\mu \mathrm{mol}, 1,00$ Äq. und 2,2'-Azobis(2-methylpropionitril) $(78,0 \mu \mathrm{l}$,

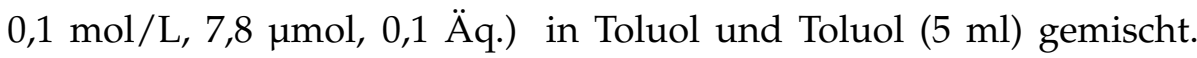
Die Mischung wurde für 10 Minuten bei $0{ }^{\circ} \mathrm{C}$ mit einem Argonstrom gespült. Anschließend wurde die Mischung für $20 \mathrm{~h}$ bei $60^{\circ} \mathrm{C}$ gerührt. Die Reaktionsmischung wurde danach in Methanol $(45 \mathrm{ml})$ gegossen und für mindestens 30 min bei 9000 Umdrehungen pro Minute zentrifugiert. Die überstehende Lösung wurde dekantiert und der Feststoff in Tetrahydrofuran $(5 \mathrm{ml})$ aufgenommen. Der Fällungs-ZentrifugationsAufnahme-Zyklus wurde 3 mal durchgeführt. Der farblose Feststoff wurde anschließend im Vakuum getrocknet. Das Zwischenprodukt (500 mg, 14,7 fmol, 1,0 Äq.) und 2,2'-Azobis(2-methylpropionitril) in N,N-Dimethylformamid (21,8 $\mu \mathrm{l}, 0,1 \mathrm{~mol} / \mathrm{l}, 2,2 \mu \mathrm{mol}, 0,15$ Äq.) wurden vorgelegt und unter Sauerstoffausschluss 1,2-Dimethoxyethan $(0,3 \mathrm{ml})$ und n-Butylacrylat $(730 \mathrm{mg}, 5,7 \mathrm{mmol}, 390$ Äq.) zugegeben. Die Reaktionsmischung wurde für $24 \mathrm{~h}$ auf $60^{\circ} \mathrm{C}$ erhitzt. Anschließend 
wurden flüchtige Komponenten im Vakuum entfernt und das Produkt erhalten.

\section{Synthese von BCP VIII}

Methylmethacrylat (5,0 ml, $48 \mathrm{mmol}, 800$ Äq.) wurde mit 4-Cyano-4-[(dodecylsulfanylthiocarbonyl)sulfanyl]-pentansäure $(23,6 \mathrm{mg}$, 59,0 $\mu \mathrm{mol}, 1,00$ Äq. und 2,2'-Azobis(2-methylpropionitril) (59,0 $\mu \mathrm{l}$, 0,1 mol/L, 5,9 $\mu \mathrm{mol}, 0,1$ Äq.) in Toluol und Toluol (5 ml) gemischt. Die Mischung wurde für 10 Minuten bei $0{ }^{\circ} \mathrm{C}$ mit einem Argonstrom gespült. Anschließend wurde die Mischung für $20 \mathrm{~h}$ bei $60^{\circ} \mathrm{C}$ gerührt. Die Reaktionsmischung wurde danach in Methanol $(45 \mathrm{ml})$ gegossen und für mindestens 30 min bei 9000 Umdrehungen pro Minute zentrifugiert. Die überstehende Lösung wurde dekantiert und der Feststoff in Tetrahydrofuran $(5 \mathrm{ml})$ aufgenommen. Der Fällungs-ZentrifugationsAufnahme-Zyklus wurde 3 mal durchgeführt. Der farblose Feststoff wurde anschließend im Vakuum getrocknet. Das Zwischenprodukt (500 mg, $12 \mu \mathrm{mol}, 1,0$ Äq.) und 2,2'-Azobis(2-methylpropionitril) in $\mathrm{N}, \mathrm{N}$-Dimethylformamid (18,2 $\mu \mathrm{l}, 0,1 \mathrm{~mol} / \mathrm{l}, 1,8 \mu \mathrm{mol}, 0,15$ Äq.) wurden vorgelegt und unter Sauerstoffausschluss 1,2-Dimethoxyethan $(0,3 \mathrm{ml})$ und n-Butylacrylat (730 mg, 5,7 mmol, 475 Äq.) zugegeben. Die Reaktionsmischung wurde für $24 \mathrm{~h}$ auf $60{ }^{\circ} \mathrm{C}$ erhitzt. Anschließend wurden flüchtige Komponenten im Vakuum entfernt und das Produkt erhalten.

\section{Synthese von BCP IX}

Methylmethacrylat (5,0 ml, $48 \mathrm{mmol}, 1000$ Äq.) wurde mit 4-Cyano-4-[(dodecylsulfanylthiocarbonyl)sulfanyl]-pentansäure $\quad(18,9 \mathrm{mg}$, 47,0 $\mu \mathrm{mol}, 1,00$ Äq. und 2,2'-Azobis(2-methylpropionitril) $(47,0 \mu \mathrm{l}$, 0,1 mol/L, 4,7 $\mu \mathrm{mol}, 0,1$ Äq.) in Toluol und Toluol (5 ml) gemischt. Die Mischung wurde für 10 Minuten bei $0{ }^{\circ} \mathrm{C}$ mit einem Argonstrom gespült. Anschließend wurde die Mischung für $20 \mathrm{~h} \mathrm{bei} 60{ }^{\circ} \mathrm{C}$ gerührt. Die Reaktionsmischung wurde danach in Methanol $(45 \mathrm{ml})$ gegossen 
und für mindestens 30 min bei 9000 Umdrehungen pro Minute zentrifugiert. Die überstehende Lösung wurde dekantiert und der Feststoff in Tetrahydrofuran $(5 \mathrm{ml})$ aufgenommen. Der Fällungs-ZentrifugationsAufnahme-Zyklus wurde 3 mal durchgeführt. Der farblose Feststoff wurde anschließend im Vakuum getrocknet. Das Zwischenprodukt (500 mg, 11,1 $\mu \mathrm{mol}, 1,0$ Äq.) und 2,2'-Azobis(2-methylpropionitril) in $N, N$-Dimethylformamid ( $16,6 \mu \mathrm{l}, 0,1 \mathrm{~mol} / 1,1,7 \mu \mathrm{mol}, 0,15$ Äq.) wurden vorgelegt und unter Sauerstoffausschluss 1,2-Dimethoxyethan $(0,3 \mathrm{ml})$ und n-Butylacrylat $(730 \mathrm{mg}, 5,7 \mathrm{mmol}, 510$ Äq.) zugegeben. Die Reaktionsmischung wurde für $24 \mathrm{~h}$ auf $60^{\circ} \mathrm{C}$ erhitzt. Anschließend wurden flüchtige Komponenten im Vakuum entfernt und das Produkt erhalten. 


\section{Anhang}

\subsection{Herleitung der Gleichung für $c_{\text {cis }}$}

Für die Gesamtkonzentration an Azo-Gruppen gilt

$$
c_{\text {ges }}=c_{\text {cis }}+c_{\text {trans }} .
$$

Durch Einsetzen des umgeformten Lambert-Beer'schen-Gesetzes und Umstellen wird

$$
\begin{aligned}
c_{\text {ges }} & =\frac{E_{\text {cis }}}{\varepsilon_{\text {cis }}}+\frac{E_{\text {trans }}}{\varepsilon_{\text {trans }}} \\
c_{\text {ges }} & =\frac{E_{\text {cis }}}{\varepsilon_{\text {cis }}}+\frac{E_{\text {ges }}-E_{\text {cis }}}{\varepsilon_{\text {trans }}} \\
c_{\text {ges }}-\frac{E_{\text {ges }}}{\varepsilon_{\text {trans }}} & =\frac{E_{\text {cis }}}{\varepsilon_{\text {cis }}}-\frac{E_{\text {cis }}}{\varepsilon_{\text {trans }}} \\
c_{\text {ges }}-\frac{E_{\text {ges }}}{\varepsilon_{\text {trans }}} & =E_{\text {cis }} \cdot\left(\frac{1}{\varepsilon_{\text {cis }}}-\frac{1}{\varepsilon_{\text {trans }}}\right) \\
E_{\text {cis }} & =\frac{c_{\text {ges }}-\frac{E_{\text {ges }}}{\varepsilon_{\text {trans }}}}{\frac{1}{\varepsilon_{\text {cis }}}-\frac{1}{\varepsilon_{\text {trans }}}}
\end{aligned}
$$

erhalten. Zuletzt wird durch $\varepsilon_{\text {cis }}$ geteilt und es folgt für $c_{\text {cis }}$ :

$$
\begin{aligned}
\frac{E_{\mathrm{cis}}}{\varepsilon_{\mathrm{cis}}} & =\frac{c_{\mathrm{ges}}-\frac{E_{\mathrm{ges}}}{\varepsilon_{\text {trans }}}}{\left(\frac{1}{\varepsilon_{\mathrm{cis}}}-\frac{1}{\varepsilon_{\text {trans }}}\right) \cdot \varepsilon_{\mathrm{cis}}} \\
c_{\mathrm{cis}} & =\frac{c_{\mathrm{ges}}-\frac{E_{\mathrm{ges}}}{\varepsilon_{\mathrm{ctans}}}}{1-\frac{\varepsilon_{\mathrm{cis}}}{\varepsilon_{\text {trans }}}}
\end{aligned}
$$

mit $\varepsilon_{\text {trans }}=4001 \mathrm{~mol}^{-1} \mathrm{~cm}^{-1}$ und $\varepsilon_{\mathrm{cis}}=15001 \mathrm{~mol}^{-1} \mathrm{~cm}^{-1}[59]$. 


\subsection{Wertetabelle für die Dichten und Dipolmomente in Abbildung 15}

Tabelle 15: Wertetabelle für die Dichten und Dipolmomente in Abbildung 15. Mit $^{*}$ markierte Werte wurden mit TINKER berechnet. ${ }^{195}$

\begin{tabular}{llcc}
\hline Polymer & Monomer & Dipolmoment / D & Dichte / $\mathrm{g} \mathrm{cm}^{-3}$ \\
\hline PB & Butadien & $0^{111}$ & $0,96^{197}$ \\
PE & Ethylen & $0^{111}$ & $0,925^{197}$ \\
PS & Styrol & $0,059^{*}$ & $1,053^{197}$ \\
PP & Propylen & $0,366^{111}$ & $0,945^{197}$ \\
PVF & Vinylfluorid & $1,43^{111}$ & $1,34^{197}$ \\
PVC & Vinylchlorid & $1,45^{111}$ & $1,391^{197}$ \\
PMA & Methylacrylat & $1,468^{*}$ & $1,224^{197}$ \\
PMMA & Methylmethacrylat & $1,682^{*}$ & $1,19^{197}$ \\
PVAc & Vinylacetat & $1,745^{*}$ & $1,191^{197}$ \\
PGA & Hydroxyessigsäure & $2,1569^{198}$ & $1,53^{197}$ \\
PEG & Ethylenglykol & $2,28^{111}$ & $1,125^{197}$ \\
POM & Formaldehyd & $2,33^{111}$ & $1,42^{197}$ \\
\hline
\end{tabular}


Anhang

\subsection{AFM-Aufnahmen von BCP III}
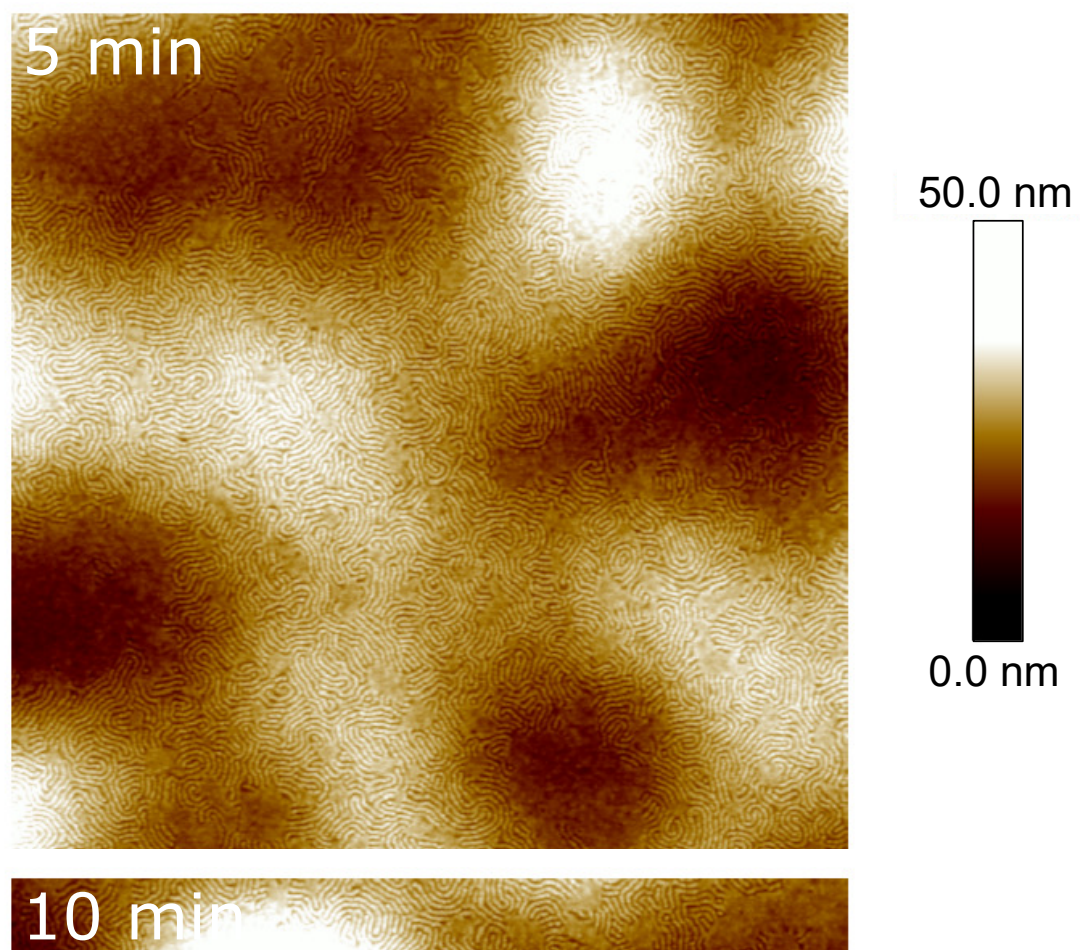

$50.0 \mathrm{~nm}$

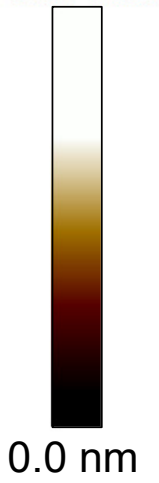


Anhang

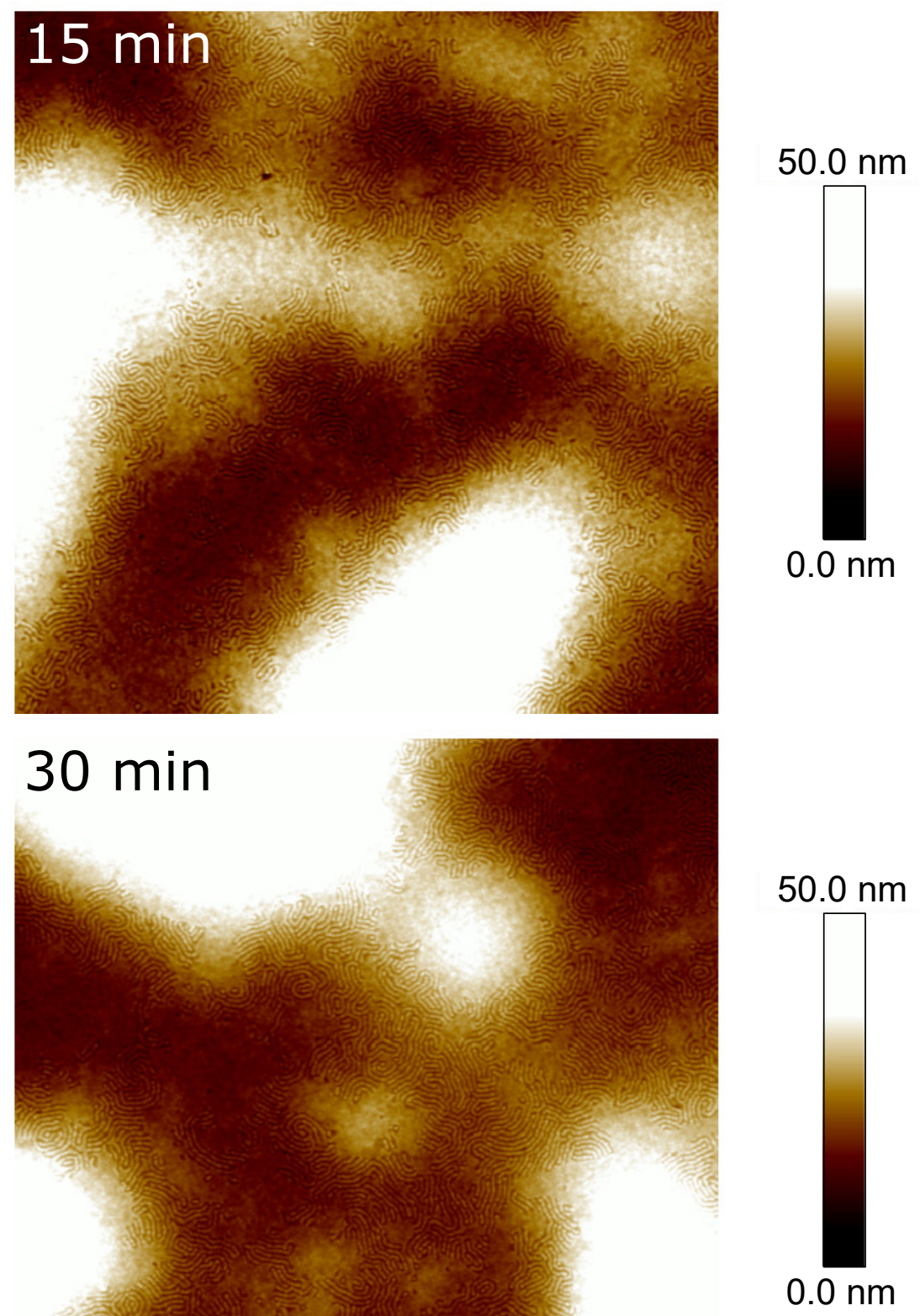


Anhang
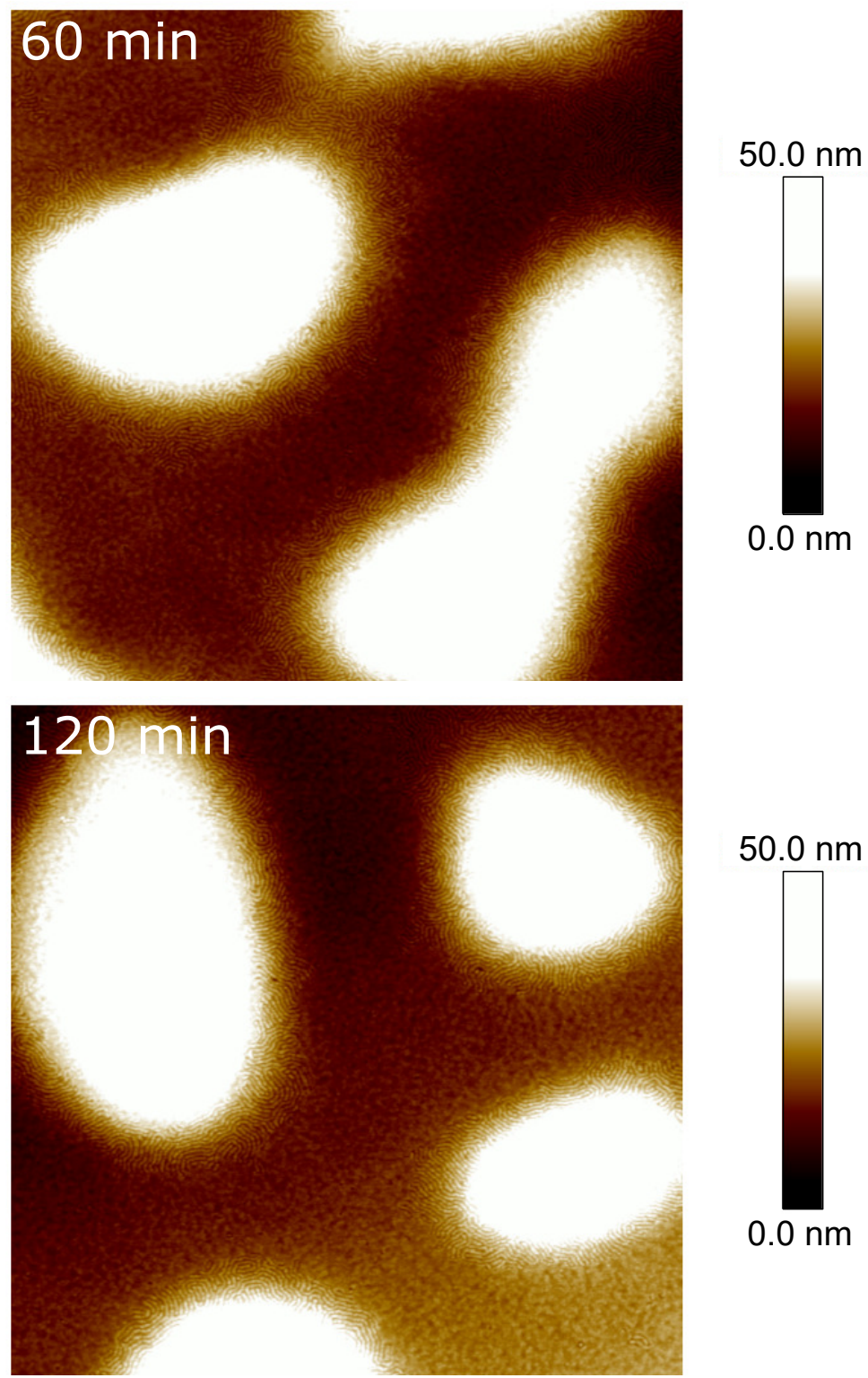

Abbildung 63: AFM-Aufnahmen des Höhenprofils von BCP III nach unterschiedlichen Temperdauern bei $180^{\circ} \mathrm{C}$. Die Proben wurden auf Si-Wafer gespincoatet. Ausschnitt: $5,3 \times 5,3 \mu \mathrm{m}$, Auflösung: $512 \times 512$ Pixel. 
Anhang

\subsection{AFM-Aufnahmen des DMT-Moduls von BCP I+20-50}

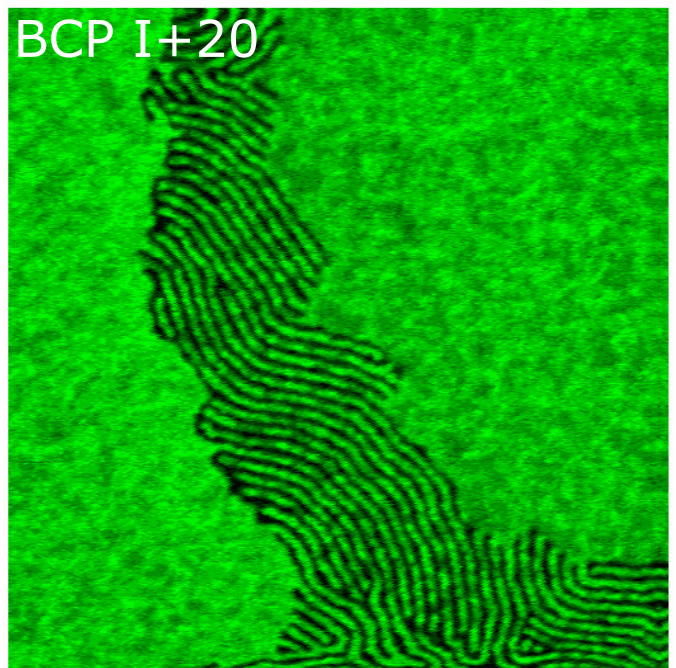

100.0 w. E.

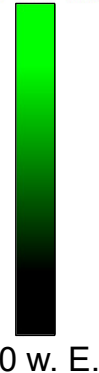

Abbildung 64: DMT-Modul der Oberfläche von BCP I+20 nach Tempern bei $180{ }^{\circ} \mathrm{C}$ für $24 \mathrm{~h}$, bestimmt durch AFM. Ausschnitt: $2,0 \times$ 2,0 $\mathrm{mm}$, Auflösung: $512 \times 512$ Pixel.

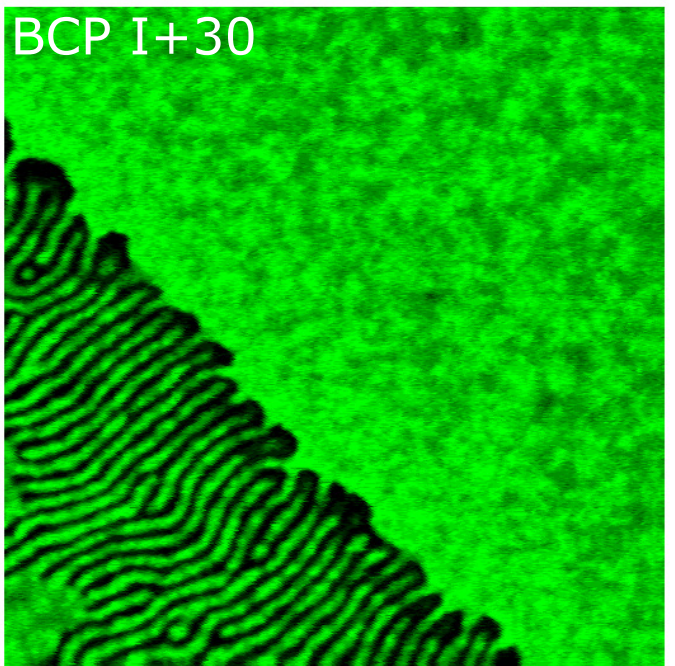

100.0 w. E.

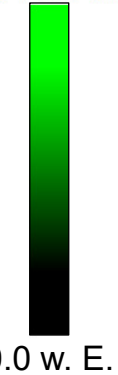

Abbildung 65: DMT-Modul der Oberfläche von BCP I+30 nach Tempern bei $180{ }^{\circ} \mathrm{C}$ für $24 \mathrm{~h}$, bestimmt durch AFM. Ausschnitt: $2,0 \times$ 2,0 $\mathrm{mm}$, Auflösung: $512 \times 512$ Pixel. 
Anhang

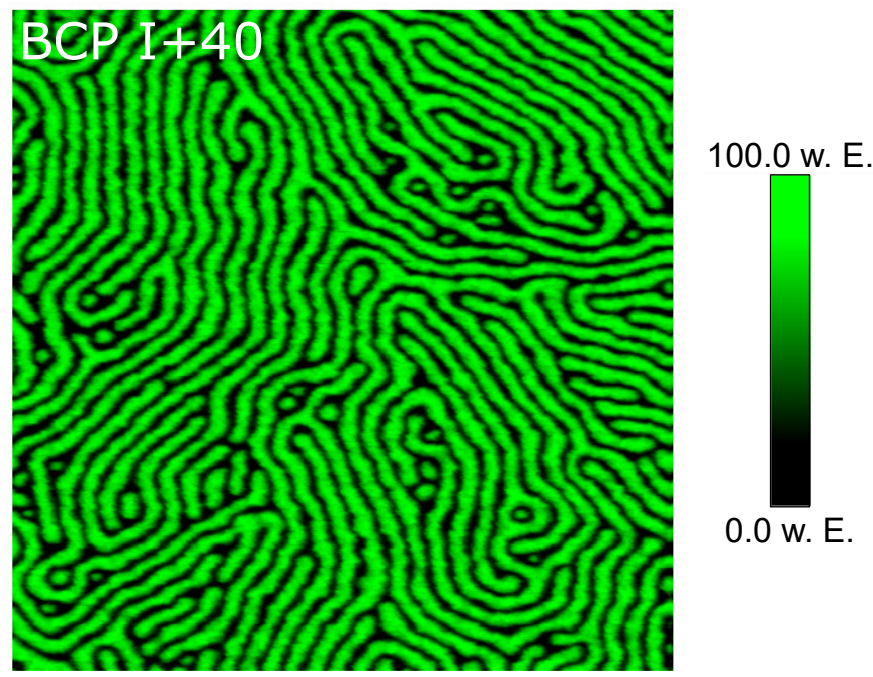

Abbildung 66: DMT-Modul der Oberfläche von BCP I+40 nach Tempern bei $180{ }^{\circ} \mathrm{C}$ für $24 \mathrm{~h}$, bestimmt durch AFM. Ausschnitt: $2,0 \times$ 2,0 $\mathrm{mm}$, Auflösung: $512 \times 512$ Pixel.

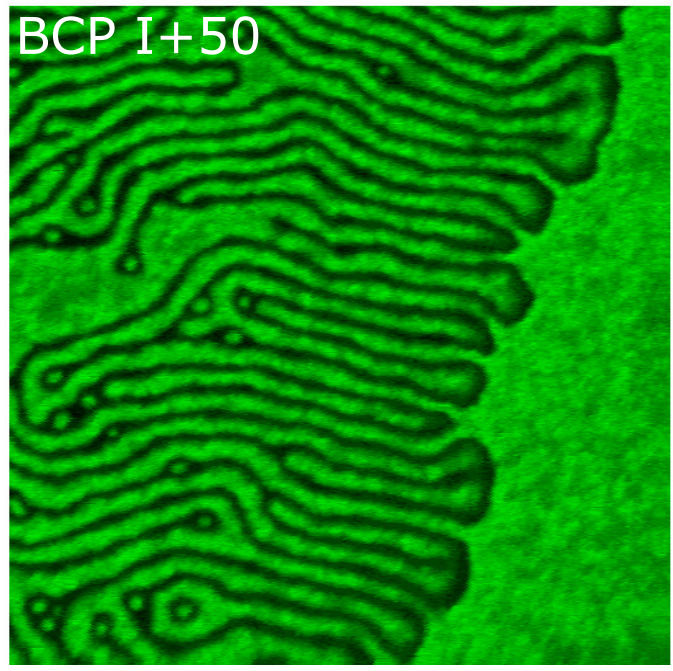

100.0 w. E.

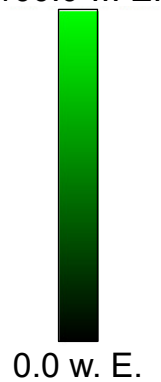

Abbildung 67: DMT-Modul der Oberfläche von BCP I+50 nach Tempern bei $180{ }^{\circ} \mathrm{C}$ für $24 \mathrm{~h}$, bestimmt durch AFM. Ausschnitt: 2,0 ×

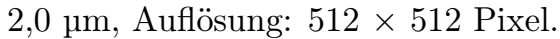




\subsection{Flory-Fox-Auftragung für PMMA I-X}

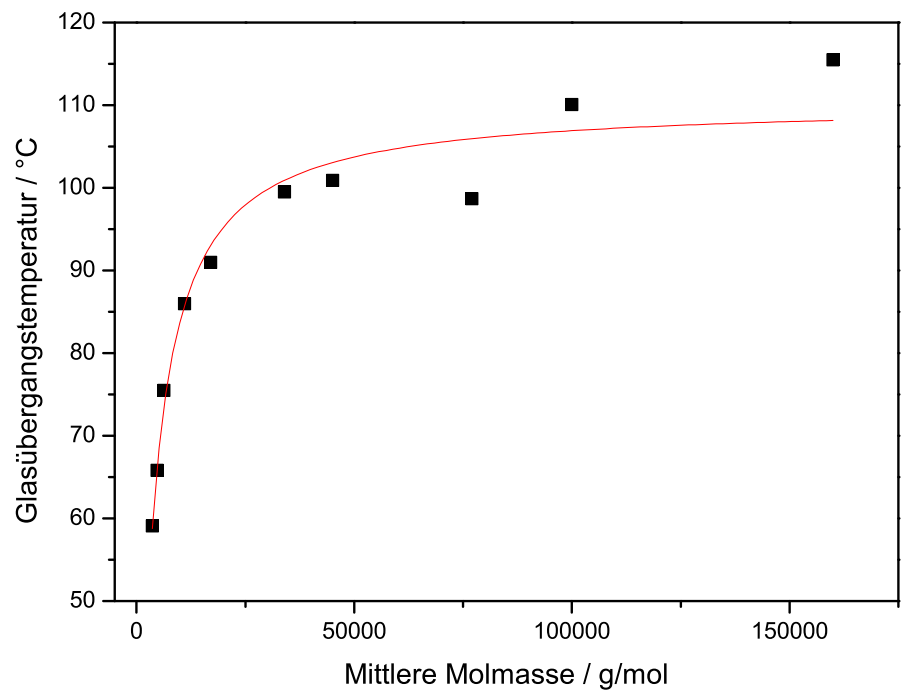

Abbildung 68: Auftragung der Glasübergangstemperatur von PMMA I-X gegen die mittlere Molmasse, sowie die Anpassung nach FloryFox (rot).

Die Glasübergangstemperaturen von PMMA I-X wurden gegen die mittlere Molmasse aufgetragen. Durch eine Anpassung gemäß der Flory-Fox-Gleichung

$$
T_{\mathrm{g}}=T_{\mathrm{g}, \infty}-\frac{K}{M_{\mathrm{n}}}
$$

wurden die Parameter $T_{\mathrm{g}, \infty}=(110 \pm 2){ }^{\circ} \mathrm{C}$ und $K=(3200 \pm 300) \frac{{ }^{\circ} \mathrm{Cg}}{\mathrm{mol}}$ bestimmt. 


\subsection{Größenverteilung der Gold-Nanopartikel}

Die Aufnahmen der Transmissionselektronenmikroskopie wurden mit dem Programm ImageJ skaliert. Die Größenverteilung wurde mit dem PSA-Makro (PSAr12) von Ralph Sperling bestimmt. Es wurden 2726 Nanopartikel analysiert.

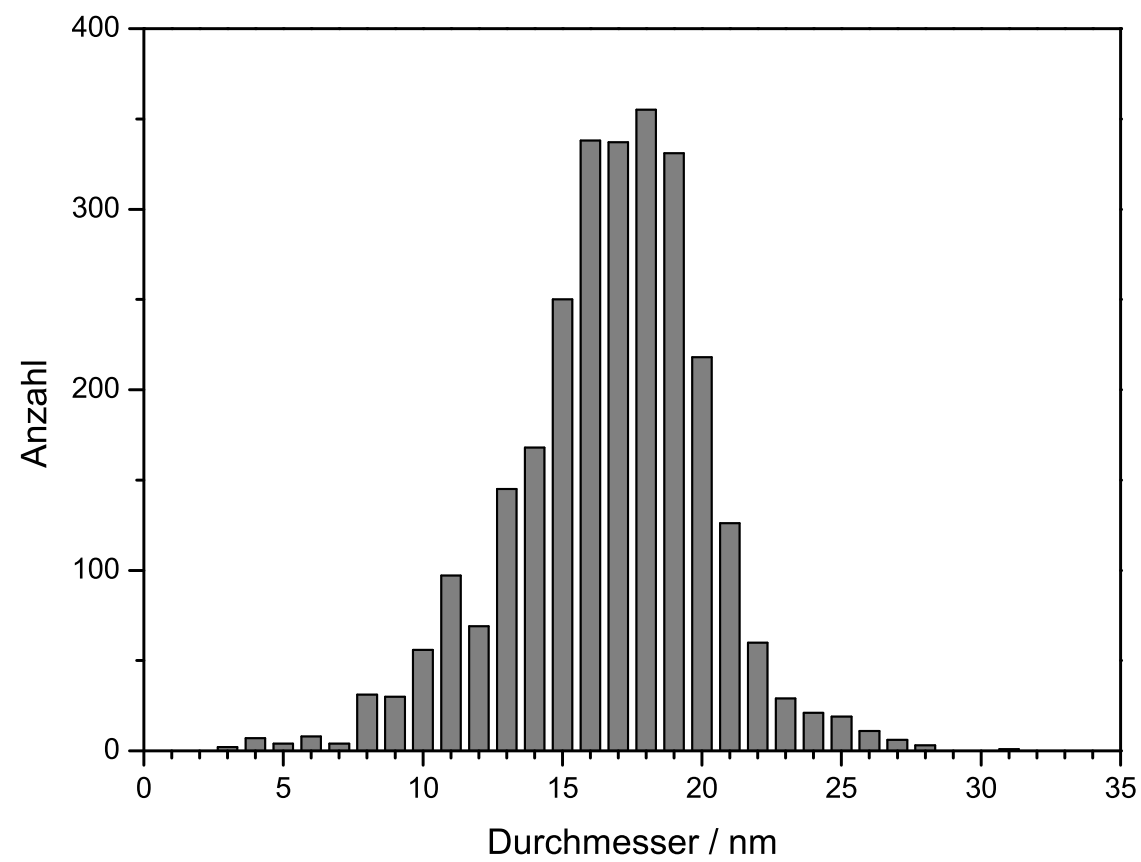

Abbildung 69: Größenverteilung der Gold-Nanopartikel aus der CitratReduktion. 


\subsection{Berechnung des Massenanteils an Gold in den funktionalisierten AuNP}

Der Massenanteil an Gold in den funktionalisierten AuNP $w_{\mathrm{Au}}$ berechnet sich durch

$$
w_{\mathrm{Au}}=\frac{m_{\mathrm{Au}}}{m_{\mathrm{Au}}+m_{\mathrm{PMMA}}} .
$$

Unter der Annahme einer idealen Kugel ergibt sich für das Volumen $V$ und die Oberfläche $O$ einer Kugel mit einem Radius $r=8 \mathrm{~nm}$

$$
\begin{aligned}
V & =\frac{4}{3} \pi r^{3}=1613 \mathrm{~nm}^{3} \\
O & =4 \pi r^{2}=804 \mathrm{~nm}^{2} .
\end{aligned}
$$

Mit der Dichte von Gold $\rho_{\mathrm{Au}}=19,3 \mathrm{~g} / \mathrm{cm}^{3}$ wird eine Masse eines Goldnanopartikels von $m_{\mathrm{Au}}=3,11 \cdot 10^{-21} \mathrm{~g}$ erhalten.

Mit der durchschnittlichen Beladungsdichte an Polymer auf einem solchen Nanopartikel ${ }^{199} \beta_{\text {PMMA }}=0,92 \mathrm{~nm}^{-2}$ wird die Masse an PMMA auf dem AuNP $m_{\text {PMMA }}$ durch

$$
m_{\mathrm{PMMA}}=\frac{\beta_{\mathrm{PMMA}} \cdot O \cdot M_{\mathrm{n}}}{N_{\mathrm{A}}}=3,11 \cdot 10^{-21} \mathrm{~g}
$$

berechnet, woraus der Massenanteil $w_{\mathrm{Au}}=0,47$ folgt. 


\section{Literatur}

(1) Carothers, W. H. Chem. Rev. 1931, 8, 353-426.

(2) Statista Weltweite und europäische Produktionsmenge von Kunststoff in den Jahren von 1950 bis 2018., https : / / de . statista . com / statistik / daten / studie / 167099 / umfrage / weltproduktion-von-kunststoff-seit-1950/, 2019.

(3) Vorsamer, B. Wie böse ist die Plastikverpackung., https://www. sueddeutsche . de / leben / nachhaltigkeit - im - supermarkt wie-boese-ist-die-plastikverpackung-1.2892418, 2016.

(4) Otto, A. Das plastikfreie Leben., https : //www . spiegel . de / wissenschaft/mensch/nachhaltigkeit-im-alltag-plastikvermeiden-a-1147204.html, 2017.

(5) Mildner, V. Warum wir dringend über Plastik in unserem Alltag nachdenken sollten., https : / / www . focus . de/perspektiven / nachhaltigkeit / nachhaltiger - leben / schwerpunkt nachhaltigkeit - stoffbeutel - und - mehrwegbecher - was bringt-der-verzicht-auf-plastik-wirklich_id_10867252 . html, 2019.

(6) Bundesregierung Dünne Plastiktüten künftig verboten., https : / / www . bundesregierung . de / breg - de / aktuelles / dunne plastiktueten-verboten-1688818, 2019.

(7) Parlament, E. Wegwerfprodukte aus Plastik: Parlament stimmt für Verbot ab 2021., https://www. europarl. europa.eu/news / de / press - room / 20190321 IPR32111 / wegwerfprodukte - aus plastik-parlament-stimmt-fur-verbot-ab-2021, 2019.

(8) Chefari, J.; Chong, Y. K.; Ercole, F.; Krstina, J.; Jeffery, J.; Le, T. P. T.; Mayadunne, R.; Meijs, G. F.; Moad, C. L.; G, M.; E, R.; H, T. S. Macromolecules 1998, 31, 5559-5562.

(9) Moad, G.; Rizzardo, E.; Thang, S. H. Aust. J. Chem. 2005, 58, $379-410$. 
(10) Moad, G.; Rizzardo, E.; Thang, S. H. Aust. J. Chem. 2006, 59, 669-692.

(11) Moad, G.; Rizzardo, E.; Thang, S. H. Aust. J. Chem. 2009, 62, 1402-1472.

(12) Moad, G.; Rizzardo, E.; Thang, S. H. Chem. Asian J. 2013, 8, 1634-1644.

(13) Huebner, D.; Rossner, C.; Vana, P. Polymer 2016, 107, 503-508.

(14) Hendrich, M.; Lewerdomski, L.; Vana, P. J. Polym. Sci. Part A Polym. Chem. 2015, 53, 2809-2819.

(15) Ebeling, B.; Eggers, S.; Hendrich, M.; Nitschke, A.; Vana, P. Macromolecules 2014, 47, 1462-1469.

(16) Abbasi, M.; Faust, L.; Milhelm, M. Adv. Mater. 2019, 31, 1806484 .

(17) Huang, K.; Rzayev, J. J. Am. Chem. Soc. 2009, 131, 6880-6885.

(18) Zhang, X.; Lian, X.; Liu, L.; Zhang, J.; Zhao, H. Macromolecules 2008, 41, 7863-7869.

(19) Rossner, C.; Glatter, O.; Vana, P. Macromolecules 2017, 50, $7344-7350$.

(20) Rossner, C.; Vana, P. Angew. Chemie Int. Ed. 2014, 53, 12639 12642 .

(21) Peng, W.; Rossner, C.; Roddatis, V.; Vana, P. ACS Macro Letters 2016, 5, 1227-1231.

(22) Schuetz, J. H.; Sandbrink, L.; Vana, P. Macromol. Chem. Phys. 2013, 214, 1484-1495.

(23) Schuetz, J. H.; Peng, W.; Vana, P. Polym. Chem. 2015, 6, 17141726 .

(24) Liu, W.; Zhang, S.; Liu, S.; Wu, Z.; Chen, H. Macromolecular Rapid Communications 2019, 40, 1900310.

(25) Tietz, K.; Finkhäuser, S.; Samwer, K.; Vana, P. Macromol. Chem. Phys. 2014, 215, 1563-1572. 
(26) Zhang, L.; Nguyen, T. L. U.; Bernhard, J.; Davis, T. P.; BarnerKowollik, C.; Stenzel, M. H. Biomacromolecules 2007, 8, 28902901.

(27) Henkel, R.; Vana, P. Macromol. Chem. Phys. 2014, 215, 182-189.

(28) Jeevanandam, J.; Barhoum, A.; Chan, Y. S.; Dufresne, A.; Danquah, M. K. Beilstein J. Nanotechnol. 2018, 9, 1050-1074.

(29) Goulet-Hanssens, A.; Eisenreich, F.; Hecht, S. Adv. Mater. 2020, 32, 1905966.

(30) Spruell, J.; Hawker, C. J. Chem. Sci. 2011, 2, 18-26.

(31) Khan, T.; Fornefeld, T.; Hübner, D.; Vana, P.; Tietze, L. Org. Lett. 2018, 20, 2007-2010.

(32) Perla, V. K.; Ghosh, S. K.; Myeni, N.; Mallick, K. Chem. Phys. Lett. 2018, 712, 112-117.

(33) Mitscherlich, E. Ann. Pharm. 1834, 2-3, 311-314.

(34) Krollpfeifer, F.; Mühlhausen, C.; Wolf, G. Annalen 1934, 508, 39-51.

(35) Hartley, G. S. Nature 1937, 140, 281.

(36) Fornefeld, T. Synthese und Analyse von Blockcopolymeren mit schaltbaren funktionellen Gruppen., MA thesis, Georg-AugustUniversität Göttingen, 2016.

(37) Bang, G.-U.; Shishido, A.; Ikeda, T. Macromol. Rapid Commun. 2007, 28, 1040-1044.

(38) Puntoriero, F.; Ceroni, P.; Balzani, V.; Bergamini, G.; Voegtle, F. J. Am. Chem. Soc. 2007, 129, 10714-10719.

(39) Parker, R. M.; Gates, J. C.; Rogers, H. L.; Smith, P. G. R.; Grossel, M. C. J. Mater. Chem. 2010, 20, 9118-9125.

(40) Qin, C.; Feng, Y.; An, H.; Cao, C.; Feng, W. ACS Appl. Mater. Interfaces 2017, 9, 4066-4073.

(41) Wang, K.; Yin, L.; Miu, T.; Liu, M.; Zhao, Y.; Chen, Y.; Zhou, N.; Zhang, W.; Zhu, X. Mater. Chem. Front. 2018, 2, 1112-1118. 
(42) Choi, Y.-J.; Kim, J.-T.; Yoon, W.-J.; Kang, D.-G.; Park, M.; Kim, D.-Y.; Lee, M.-H.; Ahn, S.-k.; Jeong, K.-U. ACS Macro Lett. 2018, 7, 576-581.

(43) Merino, E.; Ribagorda, M. Beilstein J. Org. Chem 2012, 8, 10711090.

(44) Norikane, Y.; Tamaoki, N. Org. Lett. 2004, 6, 2595-2598.

(45) Muraoka, T.; Kinbara, K.; Kobayashi, Y.; Aida, T. J. Am. Chem. Soc. 2003, 125, 5612-5613.

(46) Wen, Y.; Yi, W.; Meng, L.; Feng, M.; Jiang, G.; Yuan, W.; Zhang, Y.; Gao, H.; Jiang, L.; Song, Y. J. Phys. Chem. B 2003, 109, 14465-14468.

(47) Roling, O.; Stricker, L.; Voskuhl, J.; Lamping, S.; Ravoo, B. J. Chem. Commun. 2016, 52, 1964-1966.

(48) Ichimura, K.; Suzuki, Y.; Seki, T.; Hosoki, A.; Aoki, K. Langmuir 1988, 5, 1214-1216.

(49) Dietrich, P.; Michalik, F.; Schmidt, R.; Gahl, C.; Mao, G.; Breusing, M.; Priewitsch, B.; Elsässer, T.; Mendelssohn, R.; Weinelt, M.; Rück-Braun, K. Appl. Phys. A 2008, 93, 285-292.

(50) Kakiage, K.; Yamamura, M.; Ido, E.; Kyomen, T.; Unno, M.; Hanaya, M. Appl. Organomet. Chem. 2010, 25, 98-104.

(51) Banghart, M. R.; Mourot, A.; Fortin, D. L.; Yao, J. Z.; Kramer, R. H.; Trauner, D. Angew. Chem. Int. Ed. 2009, 48, 9097-9109.

(52) Banghart, M. R.; Borges, K.; Isacoff, E.; Trauner, D.; Kramer, R. H. Nat. Neurosci. 2004, 7, 1381-1386.

(53) Volgraf, M.; Gorostiza, P.; Nomano, R.; Kramer, R. H.; Isacoff, E.; Trauner, D. Nat. Chem. Biol. 2006, 2, 47-52.

(54) Wang, J.; Liu, H. B.; Ha, C. S. Tetrahedron 2009, 65, 9686-9689.

(55) Hunter, C. A.; Togrul, M.; Tomas, S. Chem. Commun. 2004, 108109. 
(56) Shinkai, S.; Minami, T.; Kusano, Y.; Manabe, O. J. Am. Chem. Soc. 1983, 105, 1851-1856.

(57) Jeong, K.-S.; Chang, K.-J.; An, Y.-J. Chem. Commun 2003, 1450-1451.

(58) Wang, D.; Zhao, W.; Wei, Q.; Zhao, C.; Zheng, Y. ChemPhotoChem 2018, 2, 403-415.

(59) Bandara, H. M. D.; Burdette, S. C. Chem. Soc. Rev. 2012, 41, 1809-1825.

(60) Tamai, N.; Miyasaka, H. Chem. Rev. 2000, 100, 1875-1890.

(61) Cembran, A.; Bernadi, F.; Garavelli, M.; Gagliardi, L.; Orlandi, G. J. Am. Chem. Soc. 2004, 126, 3234-3243.

(62) Satzger, H.; Sporlein, S.; Root, C.; Wachveitl, J.; Zinth, W.; Gilch, P. Chem. Phys. Lett. 2003, 372, 216-223.

(63) Beharry, A.; Sadovski, O.; Woolley, G. A. J. Am. Chem. Soc. 2011, 133, 19684-19687.

(64) Forber, C. L.; Kelusky, E. C.; Bunce, N. J.; Zerner, M. C. J. Am. Chem. Soc. 1985, 10\%, 5884-5890.

(65) Klajn, R. Pure Appl. Chem. 2010, 82, 2247-2279.

(66) Nishimura, N.; Tanaka, T.; Asano, M.; Sueishi, Y. J. Chem. Soc., Perkin Trans 2 1986, 1839-1845.

(67) Shin, D. M.; Whitten, D. G. J. Am. Chem. Soc. 1988, 110, 52065208 .

(68) Wang, R.; Iyoda, T.; Tryk, D. A.; Hashimoto, K.; Fujishima, A. J. Electroanal. Chem. 1997, 438, 213-219.

(69) Sortino, S.; Petralia, S.; Conoci, S.; di Bella, S. J. Mater. Chem. 2004, 14, 811-813.

(70) Dokic, J.; Gothe, M.; Wirth, J.; Peters, M. V.; Schwarz, J.; Hecht, S.; Saalfrank, P. J. Phys. Chem. A 2009, 113, 6763-6773.

(71) Spruell, J. M.; Hawker, C. J. Chem. Sci. 2011, 2, 18-26. 
(72) Marcetic, D.; Elezovic-Hadzic, S.; Adzic, N.; Zivic, I. J. Phys. A: Math. Theor. 2019, 52, 125001.

(73) Yamakawa, H.; Yoshizaki, T., Helical Wormlike Chains in Polymer Solutions; Springer-Verlag: Berlin, Heidelberg, 2016.

(74) Mahimwalla, Z.; Yager, K. G.; Mamiya, J.; Shishido, A.; Priimagi, A.; Barrett, C. J. Polym. Bull. 2012, 69, 967-1006.

(75) Tieke, B., Makromolekulare Chemie; Wiley-VCH: Weinheim, 2005.

(76) Hamley, I. W., The Physics of Block Copolymers; Oxford Science Publications: Oxford, New York, Tokio, 1998.

(77) Abetz, V., Block Copolymers II; Springer-Verlag: Berlin, Heidelberg, 2005.

(78) Förster, S.; Antonietti, M. Adv. Mater. 1998, 10, 195-217.

(79) Lee, K. S.; Kobayashi, S., Polymer Materials: Block-Copolymers, Nanocomposites, Organic/Inorganic Hybrids, Polymethylenes; Springer-Verlag: Berlin, Heidelberg, 2010.

(80) Huggins, M. L. J. Chem. Phys 1941, 9, 440-440.

(81) Flory, P. J. J. Chem. Phys 1941, 9, 660-660.

(82) Bates, F.; Frederikson, G. Annu. Rev. Phys. Chem. 1990, 41, $525-557$.

(83) Matsen, M. W.; Bates, F. S. Macromolecules 1996, 29, 10911098.

(84) Helfand, E.; Tagami, Y. Polym. Lett. 1971, 9, 741.

(85) Helfand, E.; Tagami, Y. J. Chem. Phys. 1971, 56, 3592.

(86) Helfand, E.; Tagami, Y. J. Chem. Phys. 1972, 57, 1812.

(87) Helfand, E. J. Chem. Phys. 1975, 62, 999.

(88) Helfand, E.; Sapse, A. M. J. Chem. Phys. 1975, 62, 1327.

(89) Helfand, E.; Wasserman, Z. R. Macromolecules 1976, 9, 879.

(90) Helfand, E.; Wasserman, Z. R. Macromolecules 1978, 11, 960. 
(91) Helfand, E.; Wasserman, Z. R. Macromolecules 1980, 13, 994.

(92) Matsen, M. W.; Schick, M. Phys. Rev. Lett. 1994, 72, 2660-2663.

(93) Wei, M.; Sun, W.; Shi, X.; Wang, Z.; Wang, Y. Macromolecules 2016, 49, 215-223.

(94) Tanaka, Y.; Hosokawa, H.; Kubota, H.; Makita, T. Int. J. Thermophys. 1991, 12, 245-264.

(95) Meier, D. J.; Company, S. D. J. Polym. Sci. Part C 1969, 26, 81-98.

(96) Albert, J. N. L.; Epps, T. H. Mater. Today 2010, 13, 24-33.

(97) Fasolka, M. J.; Mayes, A. M. Annu. Rev. Mater. Res. 2001, 31, 323-355.

(98) Tietz, K. Mikrophasenseparation von photo-vernetzbaren Blockcopolymeren in dünnen Filmen., Ph.D. Thesis, Georg-AugustUniversität Göttingen, 2014.

(99) Adams, E. Q.; Rosenstein, L. J. Am. Chem. Soc. 1914, 36, 14521473.

(100) Moore, A. L.; Shi, L. Mater. Today 2014, 17, 163-174.

(101) Han, C.; Li, Z.; Dou, S. Chinese Sci. Bull 2014, 59, 2073-2091.

(102) Park, J. G.; Cheng, Q.; Lu, J.; Bao, J.; Li, S.; Tian, Y.; Liang, Z.; Zhang, C.; Wang, B. Carbon 2012, 50, 2083-2090.

(103) Kim, H. S.; Jang, J. U.; Yu, J.; Kim, S. Y. Composites B 2015, 79, 505-512.

(104) Asadi, I.; Shafigh, P.; Hassan, Z. F. B. A.; Mahyuddin, N. B. J. Build. Eng. 2018, 20, 81-93.

(105) Burger, N.; Laachachi, A.; Ferriol, M.; Lutz, M.; Toniazzo, V.; Ruch, D. Prog. Polym. Sci. 2016, 61, 1-28.

(106) Lee, J. H.; Lee, S. H.; Choi, C. J.; Yang, S. P.; Choi, S. U. S. Int. J. Micro-Nano Scale Transf. 2010, 1, 269-322.

(107) Chen, H.; Ginzburg, V.; Yang, J.; Yang, Y.; Liu, W.; Huang, Y.; Du, L.; Chen, B. Prog. Polym. Sci 2016, 59, 41-85. 
(108) Han, Z. D.; Fina, A. Prog. Polym. Sci. 2011, 36, 914-944.

(109) Shen, S.; Henry, A.; Tong, J.; Zheng, R.; Chen, G. Nat. Nanotechnol. 2010, 5, 251-255.

(110) Chung, D. D. L. Appl. Therm. Eng. 2001, 21, 1593-1603.

(111) Lide, D. R., Handbook of Chemistry and Physics; CRC Press: Boca Raton, New York, London, Tokio, 1996.

(112) Wang, S. L.; Cheng, Y.; Wang, R. R.; Sun, J.; Gao, L. ACS Appl. Mater. Interfaces 2014, 6, 6481-6486.

(113) Pashayi, K.; Fard, H. R.; Lai, F. Y.; Iruvanti, S.; Plawsky, J.; Borca-Tasciuc, T. J. Appl. Phys. 2012, 111, 104310/1-104310.

(114) Yu, Y.; Wu, L. Z.; Zhi, J. F. Angew. Chem. Int. Ed. 2014, 53, 14326-14351.

(115) Zhang, W. B.; Xu, X. F.; Yang, J. H.; Huang, T.; Zhang, N.; Wang, Y.; Zhou, Z. W. Compos. Sci. Technol. 2015, 106, 1-8.

(116) Stankovic, S.; Dikin, D. A.; Dommett, G. H. B.; Kohlhaas, K. M.; Zimney, E. J.; Stach, E. A.; Piner, R. D.; Nguyen, S. T.; Ruoff, R. S. Nature 2006, 442, 282-286.

(117) Agari, Y.; Ueda, A.; Omura, Y.; Nagai, S. Polymer 1997, 38, 801-807.

(118) Price, D. M.; Jarratt, M. Thermochimica Acta 2002, 392, 231236.

(119) Haggenmueller, R.; Guthy, C.; Lukes, J. R.; Fische, J. E.; Winey, K. I. Macromolecules 2007, 40, 2417-2421.

(120) Henry, A.; Chen, G. Phys. Rv. Lett 2008, 101, 235502.

(121) Ho, C. Y.; Powell, R. W.; Liley, P. E. J. Phys. Chem. Ref. Data 1974, 1, 279-421.

(122) Berber, S.; Kwon, Y.-K.; Tomanek, D. Phys. Rev. Lett. 2000, 84, 4613. 
(123) Kim, Y. A.; Kamio, S.; Tajiri, T.; Hayashi, T.; Song, S. M.; Endo, M.; Terrones, M.; Dresselhaus, M. S. Appl. Phys. Lett. 2007, 90, $1-3$.

(124) Fujii, M.; Zhang, X.; Xie, H.; Takahashi, K.; Ikuta, T.; Abe, H.; Shimizu, T. Phys. Rev. Lett. 2005, 1, 065502.

(125) Huang, C.; Qian, X.; Yang, R. cond-mat.mtrl-sci 2018, 61, 1-64.

(126) Sun, H. J. Phys. Chem. B 1998, 102, 7338-7364.

(127) Sundararajan, P. R.; Kavassalis, T. A. J. Chem. Soc. 1995, 91, 2541-2549.

(128) Sun, H.; Mumby, S. J.; Maple, J. R.; Hagler, A. T. J. Am. Chem. Soc 1994, 116, 2978-2987.

(129) Xu, Y.; Wang, X.; Zhou, J.; Song, B.; Jiang, Z.; Lee, E. M.; Huberman, S.; Gleason, K.; Chen, G. Science Advances 2018, 4, 3031.

(130) Liu, J.; Yang, R. Phys. Rev. B 2012, 86, 104307.

(131) Luo, D. C.; Huang, C. L.; Huang, Z. J. Heat Transfer 2018, 140, 031302 .

(132) Ma, H.; Tian, Z. Appl. Phys. Lett. 2017, 110, 091903.

(133) Guo, Z.; Le, D.; Liu, Y.; Sun, F.; Sliwinkski, A.; Gao, H.; Burns, P. C.; Huang, L.; Luo, T. Phys. Chem. Chem. Phys. 2014, 16, 7764-7771.

(134) Chen, X. P.; Liang, Q. H.; Jiang, J. K.; Wong, C. K. Y.; Leung, S. Y. Y.; Ye, H. Y.; Yang, D. G.; Ren, T. L. Sci. Rep. 2016, 6, 20621.

(135) Kikugawa, G.; Desai, T. G.; Keblinski, P.; Ohara, T. J. Appl. Phys. 2013, 114, 034302.

(136) Zhang, L.; Ruesch, M.; Zhang, X.; Bai, Z.; Liu, L. RSC Adv. 2015, 107, 87981-87986.

(137) Kim, G.; Lee, D.; Shanker, A.; Shao, L.; Kwon, M. S.; Gidley, D.; Kim, J.; Pipe, K. P. Nat. Mater. 2015, 14, 295-300. 
(138) Mu, L.; He, J.; Li, Y.; Ji, T.; Mehra, N.; Shi, Y.; Zhu, J. J. Phys. Chem. C 2017, 121, 14204-14212.

(139) Gurau, G.; Wang, H.; Qiao, Y.; Lu, X.; Zhang, S.; Rogers, R. D. Pure Appl. Chem. 2012, 84, 745-754.

(140) Zhang, T.; Wu, X.; Luo, T. J. Phys. Chem. C 2014, 118, 21148 21159 .

(141) Luo, T.; Esfarjani, K.; Shiomi, J.; Henry, A.; Chen, G. J. Appl. Phys. 2011, 109, 074321.

(142) Xiong, X.; Yang, M.; Liu, C.; Li, X.; Tang, D. J. Appl. Phys. 2017, 122, 035104.

(143) Tonpheng, B.; Yu, J.; Andersson, O. Phys. Chem. Chem. Phys. 2011, 13, 15047-15054.

(144) Rashidi, V.; Coyle, E. J.; Sebeck, K.; Kieffer, J.; Pipe, K. P. J. Phys. Chem. B 2017, 121, 4600-4609.

(145) Yu, S.; Park, C.; Hong, S. M.; Koo, C. M. Thermochim. Acta 2014, 583, 67-71.

(146) Ni, B.; Watanabe, T.; Phillpot, S. R. J. Phys.: Condens. Matter 2009, 21, 084219.

(147) Zhao, J.; Tan, A. C.; Green, P. F. J. Mater. Chem. C 2017, 5, 10834-10838.

(148) Eslami, H.; Mohammadzadeh, L.; Mehdipour, N. J. Chem. Phys. 2012, 136, 104901.

(149) Ma, J.; Zhang, Q.; Zhang, Y.; Zhou, L.; Yang, J.; Ni, Z. Appl. Phys. Lett 2016, 109, 033101.

(150) Choy, C. L.; Wong, Y. W.; Yang, G. W.; Kanamoto, T. J. Polym. Sci., Part B: Polym. Phys. 1999, 37, 3359-3367.

(151) Supova, M.; Martynkova, G. S.; Barabaszova, K. Sci. Adv. Mater. 2011, 3, 1-25.

(152) Yuan, F. Y.; Zhang, H. B.; Li, X.; Li, X. Z.; Yu, Z. Z. Composites A 2013, 53, 137-144. 
(153) Jiang, Y.; Liu, Y.; Min, P.; Sui, G. Compos. Sci. Technol. 2017, $144,63-69$.

(154) Gu, J. W.; Zhang, Q. Y.; Dang, J.; Xie, C. Polym. Adv. Technol. 2012, 23, 1025-1028.

(155) Wang, X.; Wu, P. ACS Appl. Mater. Interfaces 2017, 9, 1993419944.

(156) Yoshihara, S.; Tokita, M.; Ezaki, T.; Nakamura, M.; Sakaguchi, M.; Matsumoto, K.; Watanabe, J. J. Appl. Polym. Sci. 2014, 131, 39896.

(157) Masoud, E. M.; El-Bellihi, A. A.; Bayoumy, W. A.; Mohamed, E. A. J. Mol. Liq. 2013, 260, 237-244.

(158) Lee, S. H.; Choi, Y. J. Nanosci. Nanotechnol. 2013, 13, 76107614 .

(159) Yu, W.; Qi, Y.; Zhou, Y.; Chen, L.; Du, H.; Xie, H. J. Appl. Polym. Sci. 2016, 133, 43242.

(160) Sun, R.; Yao, H.; Zhang, H. B.; Li, Y.; Mai, Y. W.; Yu, Z. Z. Compos. Sci. Technol. 2016, 13\%, 16-23.

(161) Choi, S.; Kim, J. Compos. B. Eng. 2013, 51, 140-147.

(162) Huang, Y.; Hu, J.; Yao, Y.; Zeng, X.; Sun, J.; Pan, G.; Sun, R.; $\mathrm{Xu}$, J.-B.; C.-P.-Wong Adv. Mater. Interfaces 2017, 4, 1700446.

(163) Patil, N.; Zhao, X.; Mishra, N. K.; Saed, M. A.; Radovic, M.; Green, M. J. ACS Appl. Mater. Interfaces 2019, 11, 46132-46139.

(164) Zhou, W.; Yu, D.; Min, C.; Fu, Y.; Guo, X. Appl. Polym. Sci. 2009, 112, 1695-1703.

(165) Wu, Y.; Yu, Z. Compos. Sci. Technol. 2015, 107, 61-66.

(166) Wu, Y.; He, Y.; Yu, Z.; Liu, X. Pro. Nat. Sci-Mater 2018, 28, 345-353.

(167) Yu, S.; Lee, J.-W.; Han, T. H.; Park, C.; Kwon, Y.; Hing, S. M.; Koo, C. M. ACS Appl. Mater. Interfaces 2013, 5, 11618-11622. 
(168) Luyt, A. S.; Molefi, J. A.; Krump, H. Polym. Degrad. Stab. 2006, 91, 1629-1636.

(169) Barani, Z.; Mohammadzadeh, A.; Geremew, A.; Coleman, C. Y.; Mangolini, L.; Kargar, F.; Balandin, A. A. Adv. Funct. Mater. 2020, 30, 1904008.

(170) Aziz, S. B.; Brza, M. A.; Mohamed, P. A.; Kadir, M. F. Z.; Hamsan, M. H.; Abdulwahid, R. T.; Woo, H. J. Results Phys. 2019, 13, 102326.

(171) Jouni, M.; Boudenne, A.; Boiteux, G.; Massardier, V.; Garnier, B.; Serghei, A. Polym. Compos. 2013, 34, 778.

(172) Li, C.; Li, Q.; Cheng, L.; Li, T.; Lu, H.; Tang, L.; Zhang, K.; Zhang, J.; Li, Z.; Yao, Y. Compos. Part A Appl. Sci. Manuf. 2017, 100, 64-70.

(173) Hammerstroem, D. W.; Burgers, M. A.; Chung, S. W.; Guliants, E. A.; Bunker, C. E.; Wentz, K. M.; Hayes, S. E.; Buckner, S. W.; Jelliss, P. A. Inorg. Chem. 2011, 50, 5054-5059.

(174) Danes, F.; Garnier, B.; Depuis, T. Int. J. Thermophys. 2003, 24, $771-784$.

(175) Zois, H.; Apekis, L.; Mamunya, Y. P. J. Appl. Polym. Sci. 2003, 88, 3013-3020.

(176) Stassi, S.; Cauda, V.; Canavese, G.; Manfredi, D.; Pirri, C. F. Eur. J. Inorg. Chem. 2012, 16, 2669-2673.

(177) Jia, X.; Listak, J.; Witherspoon, V.; Kalu, E. E.; Yang, X.; Bockstaller, M. R. Langmuir 2010, 26, 12190-12197.

(178) Razavi, R.; Zare, Y.; Rhee, K. Y. Polym. Compos. 2019, 40, 801810.

(179) Yu, A.; Ramesh, P.; Sun, X.; Bekyarova, E.; Itkis, M. E.; Haddon, R. C. Adv. Mater. 2008, 20, 4740-4744.

(180) Hu, N.; Masuda, Z.; Yan, C.; Yamamoto, G.; Fukunaga, H.; Hashida, T. Nanotechnol. 2008, 19, 215701. 
(181) Potts, J. R.; Dreyer, D. R.; Bielawski, C. W.; Ruoff, R. S. Polymer 2011, 52, 5-25.

(182) Mittal, G.; Dhand, V.; Rhee, K. Y.; Park, S.-J.; Lee, W. R. J. Ind. Eng. Chem. 2015, 21, 11-25.

(183) Du, J.; Cheng, H.-M. Macromol. Chem. Phys. 2012, 213, 10601077.

(184) Cho, H. B.; Konno, A.; Fujihara, T.; Suzuki, T.; Tanaka, S.; Jiang, W.; Suematsu, H.; Niihara, K.; Nakayama, T. Compos. Sci. Technol. 2011, 72, 112-118.

(185) Kalsoom, U.; Peristyy, A.; Nesterenko, P. N.; Pauli, B. RCS Adv. 2016, 44, 38140-38147.

(186) Nakajima, A.; Shoji, A.; Yonemori, K.; Seo, N. Jpn. J. Appl. 2016, 55, 027101.

(187) Moyer, C. A. Phys. Rev. B 1993, 47, 10079-10082.

(188) Pigard, L.; Müller, M., unveröffentlichte Ergebnisse.

(189) Fox, T. G.; Flory, P. J. J. Polym. Sci. 1954, 14, 315-319.

(190) Gaska, K.; Rybak, A.; Kapusta, C.; Sekula, R.; Siwek, R. Polym. Adv. Technol. 2015, 26, 26-31.

(191) Zhou, T.; Wang, X.; Liu, X.; Xiong, D. Carbon 2010, 48, 11711176.

(192) Nan, C. W.; Liu, G.; Lin, Y.; Li, M. Appl. Phys. Lett. 2004, 85, 3549-3551.

(193) Ebadi-Dehagani, H.; Nazempour, M. Smart Nanoparticles Technology 2012, 1, 519-540.

(194) Cao, Y.; Liang, M.; Liu, Z.; Wu, Y.; Xiong, X.; Li, C.; Wang, X.; Jiang, N.; Yu, J.; Lin, C. RCS Adv. 2016, 6, 68357-68362.

(195) Rackers, J. A.; Laury, M. L.; Lu, C.; Wang, Z.; Lagadere, L.; Piquemal, J.-P.; Rena, P.; Ponder, J. W. J. Chem. Theory Comput. 2018, 14, 5273-5289. 
(196) Balageas, D. L.; Krapez, J. C.; Cielo, P. J. Appl. Phys. 1986, 59, 348-357.

(197) Brandrup, J.; Immergut, E. H., Polymer Handbook; Wiley Interscience: New York, Chichester, Brisbane, Toronto, Singapore, 1989.

(198) Blom, C. E.; Bauder, A. Chem. Phys. Lett. 1981, 82, 492-495.

(199) Boyer, C.; Whittaker, M. R.; Chuah, K.; Liu, J.; Davis, T. P. Langmuir 2010, 26, 2721-2730. 


\section{Abkürzungsverzeichnis}

Abb.

ACCN

AFM

AIBN

AuNP

AzoHMA

AzoHOH

AzoMA

$\mathrm{AzoOH}$

AzoPMA

AzoPOH

AzoUMA

AzoUOH

BA

BCP

CDSPA

DME

DMF

DMT-Modul

DSC

ESI-MS

GPC
Abbildung

1,1'-Azobis(cyclohexancarbonitril)

Rasterkraftmikroskop(ie)

2,2'-Azobis(2-methylpropionitril)

Gold-Nanopartikel

6-(4-(Phenyldiazenyl)phenoxy)hexylmethacrylat

6-(4-(Phenyldiazenyl)phenoxy)hexan-1-ol

4-(Phenyldiazenyl)phenoxymethacrylat

4-Phenylazophenol

3-(4-(Phenyldiazenyl)phenoxy)propylmethacrylat

3-(4-(Phenyldiazenyl)phenoxy)propan-1-ol

11-(4-(Phenyldiazenyl)phenoxy)undecylmethacrylat 11-(4-(Phenyldiazenyl)phenoxy)undecan-1-ol

n-Butylacrylat

Blockcopolymer

4-Cyano-4-[(dodecylsulfanylthiocarbonyl)sulfanyl]pentansäure

1,2-Dimethoxyethan

$\mathrm{N}, \mathrm{N}$-Dimethylformamid

Modul nach Derjaguin, Müller und Topolov

Dynamische Differenzkalorimetrie

Elektrospray-Ionisations-Massenspektrometrie

Gelpermeationschromatografie 
NMR Kernspinresonanz

PAzoHMA Poly-6-(4-(Phenyldiazenyl)phenoxy)hexylmethacrylat

PAzoMA Poly-4-(Phenyldiazenyl)phenoxymethacrylat

PAzoPMA Poly-3-(4-(Phenyldiazenyl)phenoxy)propylmethacrylat

PAzoUMA Poly-11-(4-(Phenyldiazenyl)phenoxy)undecylmethacrylat

PB Polybutadien

PBA Poly-n-Butylacrylat

PDMAEMA Poly-(2-(Dimethylamino)ethylmethacrylat)

PE Polyethylen

PEG Polyethylenglykol

PGA Polyhydroxyessigsäure

PGMEA Propylenglykolmonomethyletheracetat

PMA Polymethylacrylat

PMMA Polymethylmethacrylat

POM Polyoxymethylen

PP Polypropylen

ppm Teile pro Millionen

PS Polystyrol

PVAc Polyvinylacetat

PVC Polyvinylchlorid

PVF Polyvinylfluorid

RAFT Reversibler Additions--Fragmentations Kettentransfer

SSL Strong Segregation Limit

THF Tetrahydrofuran

TTR Transiente Thermoreflektrometrie 
Abkürzungsverzeichnis

$\begin{array}{ll}\text { UV } & \text { Ultraviolett } \\ \text { UV/Vis- } & \text { Ultraviolett/Sichtbar- } \\ \text { WSL } & \text { Weak Segregation Limit }\end{array}$




\section{Variablenverzeichnis}

A

$\beta_{\text {PMMA }}$

$c$

$c_{\mathrm{Azo}}$

$c_{\mathrm{BCP} V}$

$c_{\text {cis }}$

$c_{\mathrm{cis}, 0}$

$c_{\mathrm{KV}}$

$c_{p}$

$d$

$d_{\mathrm{Azo}}$

$d_{\mathrm{BA}}$

$d_{\mathrm{h}}$

$d_{\mathrm{MPS}}$

$d_{\mathrm{V}}$

$\delta$

$Ð$

$\varepsilon$

$\varepsilon_{\mathrm{AA}}$

$\varepsilon_{\mathrm{Azo}}$

$\varepsilon_{\text {cis }}$

$\varepsilon_{\mathrm{KV}}$

$\varepsilon_{\text {trans }}$ präexponentieller Arrhenius-Faktor

Beladungsdichte eines Gold-Nanopartikels mit PMMA

Konzentration

Konzentration der Azobenzol-Gruppen

Konzentration von BCP V

Konzentration des cis-Isomers

Konzentration des $c i$-Isomers am Zeitpunkt $t=0$

Konzentration von Kristallviolett

Wärmekapazität bei konstantem Druck

Schichtdicke

Breite der PAzoPMA-Phase

Breite der PBA-Phase

Abstand der horizontal angeordneten Lamellen

Breite des mikrophasenseparierten Bereiches

Abstand der vertikal angeordneten Lamellen

chemische Verschiebung

Dispersität

Extinktionskoeffizient

Interaktionsenergie zwischen Monomer A und A

Extinktionskoeffizient von Azobenzol

Extinktionskoeffizient des cis-Isomers

Extinktionskoeffizient von Kristallviolett

Extinktionskoeffizient des trans-Isomers 
E $\quad$ Extinktion

$E_{\mathrm{A}} \quad$ Aktivierungsenergie

$E_{\text {cis }} \quad$ Extinktion des cis-Isomers

$E_{\text {ges }} \quad$ Extinktion eines gesamten Systems

$E_{\text {trans }} \quad$ Extinktion des trans-Isomers

$f_{\mathrm{A}} \quad$ Volumenanteil von Komponente A

$f_{\mathrm{B}} \quad$ Volumenanteil von Komponente B

$\Delta G_{\mathrm{BCP}} \quad$ Gibbs-Energie eines Blockcopolymers

$\Delta h \quad$ Höhendifferenz der Mikrophasen

$\Delta h_{\text {rel }} \quad$ relative Schichtdickenabnahme

$\Delta H_{\mathrm{m}} \quad$ Mischungsenthalpie

$I_{\text {Azo }} \quad$ Integral des NMR-Signals bei 7,73 ppm

$I_{\mathrm{BA}} \quad$ Integral des NMR-Signals bei 3,59 ppm

$I_{\mathrm{Me}} \quad$ Integral des NMR-Signals bei 0,86 ppm

I MMA Integral des NMR-Signals bei 4,03 ppm

$\kappa \quad$ Wärmeleitfähigkeit

$\kappa_{0} \quad$ Normierungsfaktor der Wärmeleitfähigkeit in Simulationen

$\kappa_{\mathrm{e}} \quad$ elektronischer Anteil der Wärmeleitfähigkeit

$\kappa_{\mathrm{p}} \quad$ phononischer Anteil der Wärmeleitfähigkeit

$k \quad$ Geschwindigkeitskoeffizient der Isomerisierungsreaktion

$K \quad$ Stoffkonstante in der Flory-Fox-Gleichung 


\begin{tabular}{|c|c|}
\hline$\lambda$ & Wellenlänge \\
\hline$\lambda_{\mathrm{x}}$ & Parameter für die Simulation der Wärmeleitfähigkeit \\
\hline & in Polymeren \\
\hline$l$ & mittlere freie Weglänge eines Phonons \\
\hline$L_{0}$ & Lorentz-Konstante \\
\hline$l_{\mathrm{p}}$ & Persistenzlänge \\
\hline$m_{\mathrm{x}}$ & Masse von $x$ \\
\hline$M$ & molare Masse \\
\hline$M_{\mathrm{n}}$ & zahlengemittelte molare Masse \\
\hline$M_{\mathrm{Azo}}$ & molare Masse von AzoPMA \\
\hline$M_{\mathrm{BA}}$ & molare Masse von BA \\
\hline$n_{\mathrm{BA}}$ & Anzahl an Monomereinheiten im PBA-Block \\
\hline$O$ & Oberfläche \\
\hline$p$ & Druck \\
\hline$Q$ & Wärmestrom \\
\hline$r$ & Radius \\
\hline$R$ & universelle Gaskonstante \\
\hline$\left\langle R^{2}\right\rangle$ & mittlerer quadratischer End-zu-End-Abstand \\
\hline$\sigma_{\mathrm{e}}$ & elektrische Leitfähigkeit \\
\hline$\Delta S_{\mathrm{k}}$ & Konformationsentropie \\
\hline
\end{tabular}


Variablenverzeichnis

$\begin{array}{ll}t & \text { Zeit } \\ T & \text { Temperatur } \\ T_{\mathrm{g}} & \text { Glasübergangstemperatur } \\ T_{\mathrm{g}, \infty} & \text { Glasübergangstemperatur einer unendlich langen Kette } \\ T_{\mathrm{ODT}} & \text { Ordnungs-Unordnungs-Übergangstemperatur } \\ V & \text { Volumen } \\ & \\ w_{\mathrm{Au}} & \text { Gewichtsanteil von Gold } \\ w_{\mathrm{Azo}} & \text { Gewichtsanteil von PAzoPMA } \\ w_{\mathrm{BA}} & \text { Gewichtsanteil von PBA } \\ w_{\mathrm{KV}} & \text { Gewichtsanteil von Kristallviolett } \\ & \\ \chi & \text { Interaktionsparameter nach Flory und Huggins } \\ \chi_{\mathrm{Azo}} & \text { Stoffmengenanteil an PAzoPMA } \\ \chi_{\mathrm{BA}} & \text { Stoffmengenanteil an PBA } \\ z & \end{array}$




\section{Abbildungsverzeichnis}

1 Kunststoffproduktion pro Jahr in Millionen Tonnen, weltweit und in Europa. . . . . . . . . . . . . . . . . . 1

2 Allgemeine Struktur eines RAFT-Agens. . . . . . . . . . 5

3 UV/Vis-Spektren der photoschaltbaren Monomere AzoMA (A), AzoPMA (B), AzoHMA (C) und AzoUMA (D), in trans- (schwarz) und cis-Konfiguration (rot) in THF. 11

4 Ausschnitt der $\mathrm{n} \rightarrow \pi^{*}$-Bande aus den zeitaufgelösten $\mathrm{UV} /$ Vis-Spektren von AzoMA in THF bei $60^{\circ} \mathrm{C}$. Die Abnahme der Extinktion wird durch einen Pfeil verdeutlicht. 12

5 Auftragung von $\ln \left(\frac{c_{\mathrm{cis}}}{c_{\mathrm{cis}, 0}}\right)$ gegen $t$ für AzoMA in THF bei $60{ }^{\circ} \mathrm{C} \ldots \ldots \ldots \ldots \ldots$

6 Auftragung der Geschwindigkeitskoeffizienten der thermischen Isomerisierung von AzoMA gegen das Dipolmoment des Lösungsmittels bei verschiedenen Temperaturen. . . . . . . . . . . . . . . . . 15

7 Auftragung der logarithmierten Geschwindigkeitskoeffizienten der Isomerisierung gegen die reziproke Temperatur für AzoMA in verschiedenen Lösungsmitteln. . . . . . 16

8 UV/Vis-Spektren der photoschaltbaren Polymere PAzoMA (A), PAzoPMA (B), PAzoHMA (C) und PAzoUMA (D), in trans- (schwarz) und cis-Konfiguration (rot) in THF. 18

9 Auftragung der Geschwindigkeitskoeffizienten der Isomerisierung gegen die Anzahl der Kohlenstoffatome im verwendeten Linker für photoschaltbare Monomere (oben) und photoschaltbare Polymere (unten) bei verschiedenen Temperaturen. . . . . . . . . . . . . . . . . . 20 
10 Auftragung der logarithmierten Geschwindigkeitskoeffizienten der Isomerisierung gegen die reziproke Temperatur für photoschaltbare Monomere (oben) und photoschaltbare Polymere (unten) mit verschiedenen Längen des verwendeten Linkers. . . . . . . . . . . . . . . . . 21

11 Geschwindigkeitskoeffizienten der thermischen Isomerisierung von verschiedenen photoschaltbaren Mono- und Polymeren in THF in Abhängigkeit von der Temperatur, AzoMA und PAzoMA (oben, links), AzoPMA und PAzoPMA (oben rechts), AzoHMA und PAzoHMA (unten links) sowie AzoUMA und PAzoUMA (unten rechts). . . 24

12 Geschwindigkeitskoeffizienten der thermischen Isomerisierung von verschiedenen photoschaltbaren Mono- und Polymeren in THF in Abhängigkeit von der Anzahl an Kohlenstoffatomen im verwendeten Linker bei verschiedenen Temperaturen.

13 Schichtdicken von photoschaltbaren Polymerfilmen aus PAzoMA (Oben), PAzoPMA (Mitte) und PAzoHMA (Unten) im Laufe eines Bestrahlungszyklus. . . . . . . . . . .

14 Schichtdicke eines PAzoPMA-Dünnfilmes in Abhängigkeit von der Anzahl an Bestrahlungszyklen. . . . . . . . . 30

15 Auftragung der Dichte einiger Polymere gegen das Dipolmoment der zugehörigen Monomere. PE: Polyethylen, PB: Polybutadien, PP: Polypropylen, PS: Polystyrol, PMA: Polymethylacrylat, PMMA: Polymethylmethacrylat, PVAc: Polyvinylacetat, PEG: Polyethylenglykol, PVF: Polyvinylfluorid, PVC: Polyvinylchlorid, POM: Polyoxymethylen, PGA: Polyhydroxyessigsäure. Die zugehörige Wertetabelle und Quellen sind in Kapitel 7.2 gezeigt. 31

16 Schematische Darstellung von verschiedenen Blockcopolymerarten ${ }^{36} \ldots \ldots \ldots \ldots \ldots \ldots$ 
17 Schematische Darstellung eines eindimensionalen Konzentrationsprofils gegen die Querschnittskooordinate $x$ eines in lamellarer Morphologie mikrophasenseparierten Blockcopolymers am Weak Segregation Limit (schwarz) und am Strong Segregation Limit (rot). ${ }^{36} \quad$. . . . 37

18 Schematische Darstellung der Grenzfläche (grün) zwischen zwei Phasen eines mikrophasenseparierten Blockcopolymers am WSL (links) und SSL (rechts). ${ }^{36} \ldots$. . . 38

19 Theoretisches Phasendiagramm eines Blockcopolymers nach Matsen und Bates ${ }^{83}$ in Abhängigkeit von Volumenanteil der Komponenten und $\chi N$. Die Buchstaben stehen für die Bezeichnung der jeweiligen Phase: Dicht gepackte Kugeln $\left(S^{*}\right)$, kubisch innenzentrierte Kugeln (S), hexagonal angeordnete Zylinder (Z), Gyroid (G) sowie Lamellen (L). ${ }^{36}$.

20 Schematische Darstellung der unterschiedlichen Morphologien der Mikrophasenseparation. Von links nach rechts: Kugeln, Zylinder, Gyroid, Lamellen. ${ }^{36}$. . . . . . . 39

21 Schematische Darstellung des Einflusses des Volumenanteils von Komponente A (orange) $f_{\mathrm{A}}$ auf die Form der Phasengrenzfläche (schwarz) und der Geometrie der gebildeten Morphologie der Mikrophasenseparation. ${ }^{36}$. . .

22 Aus dem GP-Chromatogramm berechnete normierte Molmassenverteilungen vom Homopolymer PAzoPMA sowie den Blockcopolymeren BCP II-IV. . . . . . . . . . . 42

$23{ }^{1} \mathrm{H}-\mathrm{NMR}-S p e k t r e n$ von BCP II-IV in $\mathrm{CD}_{2} \mathrm{Cl}_{2} \ldots \ldots \ldots$

24 AFM-Aufnahmen der Höhenprofile von BCP II-IV. Die Proben wurden auf Si-Wafer gespincoatet. Ausschnitt: 2 $\times 2 \mu \mathrm{m}$, Auflösung: $512 \times 512$ Pixel. 
25 AFM-Aufnahmen der Höhenprofile von BCP III nach unterschiedlich langem Tempern bei $180^{\circ} \mathrm{C}$. Ausschnitt: 5,3 $\times 1,2 \mu \mathrm{m}$, Auflösung: $512 \times 118$ Pixel.

26 AFM-Aufnahmen des Höhenprofils, Ausschnitt: $16 \times$ $16 \mu \mathrm{m}$ (Oben), Ausschnitt: 5,3 × 5,3 $\mu \mathrm{m}$ (Mitte) und DMTModul, Ausschnitt: 5,3 × 5,3 $\mu \mathrm{m}$ (Unten) von BCP II nach Tempern bei $180^{\circ} \mathrm{C}$ für $24 \mathrm{~h}$. Auflösung: $512 \times 512$ Pixel.

27 AFM-Aufnahmen des Höhenprofils, Ausschnitt: $16 \times$ $16 \mu \mathrm{m}$ (Oben), Ausschnitt: 5,3 × 5,3 $\mu \mathrm{m}$ (Mitte) und DMTModul, Ausschnitt: 5,3 × 5,3 $\mu \mathrm{m}$ (Unten) von BCP III nach Tempern bei $180^{\circ} \mathrm{C}$ für $24 \mathrm{~h}$. Auflösung: $512 \times 512$ Pixel. $\quad 50$

28 AFM-Aufnahmen des Höhenprofils, Ausschnitt: $16 \times$ $16 \mu \mathrm{m}$ (Oben), Ausschnitt: 5,3 × 5,3 $\mu \mathrm{m}$ (Mitte) und DMTModul, Ausschnitt: 5,3 ×5,3 $\mu \mathrm{m}$ (Unten) von BCP IV nach Tempern bei $180^{\circ} \mathrm{C}$ für $24 \mathrm{~h}$. Auflösung: $512 \times 512$ Pixel.

29 Dreidimensionale Darstellung der Oberfläche von BCP II aus der AFM-Aufnahme des Höhenprofils, Ausschnitt: $16 \times 16 \mu \mathrm{m}$.

30 Höhenprofil eines Querschnitts durch vertikal angeordnete Lamellen in BCP II. Die Breite des PAzoPMABlockes $d_{\mathrm{Azo}}$ ist rot, die Breite des PBA-Blockes $d_{\mathrm{BA}}$ ist blau, die Höhendifferenz $\Delta h$ grün und der Lamellenabstand $d_{\mathrm{v}}$ lila markiert.

31 Aus dem GP-Chromatogramm berechnete normierte Molmassenverteilungen der Homopolymere PAzoPMA und PBA. . . . . . . . . . . . . . . . . . 55

32 AFM-Aufnahmen des Höhenprofils von BCP I+10 vor dem Tempern. Ausschnitt: 2,0 × 2,0 $\mu \mathrm{m}$, Auflösung: 512 $\times 512$ Pixel. 
33 DMT-Modul der Oberfläche von BCP I+10 nach Tempern bei $180^{\circ} \mathrm{C}$ für $24 \mathrm{~h}$, bestimmt durch AFM. Ausschnitt: 2,0 $\times 2,0 \mu \mathrm{m}$, Auflösung: $512 \times 512$ Pixel.

34 AFM-Aufnahmen des Höhenprofils von BCP I+10 nach Tempern bei $180{ }^{\circ} \mathrm{C}$ für $24 \mathrm{~h}$. Ausschnitt: $16 \times 16 \mu \mathrm{m}$ (Oben), 5,3 × 5,3 $\mu \mathrm{m}$ (Mitte) und 2,0 × 2,0 $\mu \mathrm{m}$ (Unten), Auflösung: $512 \times 512$ Pixel.

35 AFM-Aufnahmen des Höhenprofils von BCP I+20 nach Tempern bei $180{ }^{\circ} \mathrm{C}$ für $24 \mathrm{~h}$. Ausschnitt: $16 \times 16 \mu \mathrm{m}$ (Oben), 5,3 × 5,3 $\mu \mathrm{m}$ (Mitte) und 2,0 × 2,0 $\mu \mathrm{m}$ (Unten), Auflösung: $512 \times 512$ Pixel. . . . . . . . . . . . . . . . . 60

36 AFM-Aufnahmen des Höhenprofils von BCP I+30 nach Tempern bei $180{ }^{\circ} \mathrm{C}$ für $24 \mathrm{~h}$. Ausschnitt: $16 \times 16 \mu \mathrm{m}$ (Oben), 5,3 × 5,3 $\mu \mathrm{m}$ (Mitte) und 2,0 × 2,0 $\mu \mathrm{m}$ (Unten), Auflösung: $512 \times 512$ Pixel.

37 AFM-Aufnahmen des Höhenprofils von BCP I+40 nach Tempern bei $180{ }^{\circ} \mathrm{C}$ für $24 \mathrm{~h}$. Ausschnitt: $16 \times 16 \mu \mathrm{m}$ (Oben), 5,3 × 5,3 $\mu \mathrm{m}$ (Mitte) und 2,0 × 2,0 $\mu \mathrm{m}$ (Unten), Auflösung: $512 \times 512$ Pixel. . . . . . . . . . . . . . . . 62

38 AFM-Aufnahmen des Höhenprofils von BCP I+50 nach Tempern bei $180{ }^{\circ} \mathrm{C}$ für $24 \mathrm{~h}$. Ausschnitt: $16 \times 16 \mu \mathrm{m}$ (Oben), 5,3 × 5,3 $\mu \mathrm{m}$ (Mitte) und 2,0 × 2,0 $\mu \mathrm{m}$ (Unten), Auflösung: $512 \times 512$ Pixel. . . . . . . . . . . . . . . . 63

39 Auftragung des horizontalen Lamellenabstandes von BCP I sowie BCP I+10-BCP I+50 gegen die Menge an zugegebenem Homopolymer, sowie berechnete hypothetische Zunahme des horizontalen Lamellenabstandes, ausgehend vom Wert von BCP I. . . . . . . . . . . . . . . . 64 
40 Auftragung der experimentell ermittelten Strukturparameter von BCP I sowie BCP I+10-50 gegen die Menge an zugegebenem Homopolymer, sowie berechnete hypothetische Zunahme der Strukturparameter, ausgehend vom Wert von BCP I. . . . . . . . . . . . . . . . . . . . . . 65

41 Beispiele für auftretende Defekte im Höhenprofil von BCP I+40. Ausschnitt: 2,0 × 2,0 $\mu \mathrm{m}$, Auflösung: $512 \times 512$ Pixel. Die verschiedenen Defektklassen sind Sackgassen (grün), Kreuzungen (rot) und Bögen (blau). Es sind nicht alle auftretenden Defekte markiert. . . . . . 70

42 Struktur von Kristallviolett. . . . . . . . . . . . . . . . . . 71

43 Schematische Darstellung der Befüllung von Blockcopolymeraggregaten aus PAzoPMA- $b$ PDMAEMA (orange-blau) mit Kristallviolett (violett). . . 72

44 UV/Vis-Spektren von PAzoPMA- $b$-PDMAEMA (orange), der befüllten Polymeraggregate (schwarz), der überstehenden Lösung nach dem Zentrifugieren (blau) und von reinem Kristallviolett (violett). . . . . . . . 73

45 Schematische Darstellung des Wärmetransportes durch Vibration in einem Material. ${ }^{105} \ldots$. . . . . . . . . . . 81

46 Schematische Darstellung des Wärmetransportes durch Vibration in einem Polymer. Warme Teilchen sind dabei in rot, kalte Teilchen in grün dargestellt. ${ }^{105}$

47 Schematische Darstellung des Wärmetransportes in Systemen mit verschiedenen Defekten: Punktdefekt (Links), Versetzung (Mitte) und Korngrenze (Rechts). Warme Teilchen sind dabei in rot, kalte Teilchen in grün dargestellt. ${ }^{105} \ldots \ldots \ldots \ldots \ldots$. . . . . . . 82

48 Ausschnitt aus der Struktur von Diamant. . . . . . . . . . 84

49 Ausschnitt aus der Struktur von Graphen und Kohlenstoffnanoröhrchen. . . . . . . . . . . . . . . . . . . . . . 84 
50 Auftragung der normierten Oberflächentemperatur des Kupferfilms gegen die Zeit nach dem Heizpuls für transPAzoPMA und cis-PAzoPMA.

51 Beispiele möglicher $\pi-\pi$-Wechselwirkungen zwischen Azobenzolgruppen in PAzoPMA.

52 Simulierte Wärmeleitfähigkeit von PMMA in Abhängigkeit von der Molmasse für verschiedene Werte des Parameters $\lambda_{\mathrm{x}}$. Die Simulation wurde von Louis Pigard am Institut für Theoretische Physik der Universität Göttingen durchgeführt. ${ }^{188}$

53 Auftragung der Wärmeleitfähigkeit der PMMADünnfilme gegen die mittlere Molmasse des Polymers.

54 Vergleich der Simulation mit den experimentellen Daten der Wärmeleitfähigkeit von PMMA mit unterschiedlicher Molmasse. . . . . . . . . . . . . . . . . . . . . . . . 93

55 TEM-Aufnahmen der PMMA-funktionalisierten AuNP in verschiedenen Vergrößerungen. . . . . . . . . . . . 9 95

56 Auftragung der normierten Oberflächentemperatur des Kupfers gegen die Zeit nach dem Heizpuls bei den Messungen der transienten Thermoreflektometrie der reinen PMMA-Matrix sowie des Gold-PMMANanokomposites. . . . . . . . . . . . . . . . . . . . 96

$57{ }^{1} \mathrm{H}-\mathrm{NMR}-\mathrm{Spektren}$ von PMMA (schwarz) und PMMA-bPBA (rot) in $\mathrm{CDCl}_{3} . \ldots \ldots$

58 AFM-Aufnahmen der Höhenprofile von BCP VI nach thermischem Tempern (oben) und Tempern in ToluolAtmosphäre (unten). Die Proben wurden auf Si-Wafer gespincoatet. Ausschnitt: $2 \times 2 \mu \mathrm{m}$, Auflösung: $512 \times$ 512 Pixel. . . . . . . . . . . . . . . . . . . . . 100 
59 AFM-Aufnahmen der Höhenprofile von BCP VII nach thermischem Tempern (oben) und Tempern in ToluolAtmosphäre (unten). Die Proben wurden auf Si-Wafer gespincoatet. Ausschnitt: $2 \times 2 \mu \mathrm{m}$, Auflösung: $512 \times$ 512 Pixel.

60 AFM-Aufnahmen der Höhenprofile von BCP VIII nach thermischem Tempern (oben) und Tempern in ToluolAtmosphäre (unten). Die Proben wurden auf Si-Wafer gespincoatet. Ausschnitt: $2 \times 2 \mu \mathrm{m}$, Auflösung: $512 \times$ 512 Pixel.

61 AFM-Aufnahmen der Höhenprofile von BCP IX nach thermischem Tempern (oben) und Tempern in ToluolAtmosphäre (unten). Die Proben wurden auf Si-Wafer gespincoatet. Ausschnitt: $2 \times 2 \mu \mathrm{m}$, Auflösung: $512 \times$ 512 Pixel.

62 Wärmeleitfähigkeit der Blockcopolymere BCP VI-IX in Abhängigkeit von der Ausrichtung der Zylinder und der mittleren Molmasse.

63 AFM-Aufnahmen des Höhenprofils von BCP III nach unterschiedlichen Temperdauern bei $180{ }^{\circ} \mathrm{C}$. Die Proben wurden auf Si-Wafer gespincoatet. Ausschnitt: 5,3 $\times$ 5,3 m, Auflösung: $512 \times 512$ Pixel. . . . . . . . . . . . . . 141

64 DMT-Modul der Oberfläche von BCP I+20 nach Tempern bei $180^{\circ} \mathrm{C}$ für $24 \mathrm{~h}$, bestimmt durch AFM. Ausschnitt: 2,0 $\times$ 2,0 $\mu \mathrm{m}$, Auflösung: $512 \times 512$ Pixel.

65 DMT-Modul der Oberfläche von BCP I+30 nach Tempern bei $180^{\circ} \mathrm{C}$ für $24 \mathrm{~h}$, bestimmt durch AFM. Ausschnitt: 2,0 $\times$ 2,0 $\mu \mathrm{m}$, Auflösung: $512 \times 512$ Pixel.

66 DMT-Modul der Oberfläche von BCP I+40 nach Tempern bei $180^{\circ} \mathrm{C}$ für $24 \mathrm{~h}$, bestimmt durch AFM. Ausschnitt: 2,0 $\times 2,0 \mu \mathrm{m}$, Auflösung: $512 \times 512$ Pixel. 
67 DMT-Modul der Oberfläche von BCP I+50 nach Tempern bei $180^{\circ} \mathrm{C}$ für $24 \mathrm{~h}$, bestimmt durch AFM. Ausschnitt: 2,0 $\times 2,0 \mu \mathrm{m}$, Auflösung: $512 \times 512$ Pixel. . . . . . . . . . . 143

68 Auftragung der Glasübergangstemperatur von PMMA I$X$ gegen die mittlere Molmasse, sowie die Anpassung nach Flory-Fox (rot). . . . . . . . . . . . . . . . . . . . . 144

69 Größenverteilung der Gold-Nanopartikel aus der CitratReduktion. . . . . . . . . . . . . . . . . . . . . 145 